\title{
Investigating the hydration and structural changes of molecular organic materials under high-pressure conditions
}

\author{
Dissertation \\ for the award of the degree \\ "Doctor of Philosophy" \\ Ph.D. Division of Mathematics and Natural Sciences \\ of the Georg-August-Universität Göttingen \\ within the doctoral program Geology / Geography \\ of the Georg-August University School of Sciences (GAUSS)
}

\author{
submitted by \\ Rubén Granero-García \\ from Zaragoza (Spain)
}

Göttingen, 2016 


\section{Thesis committee}

Dr. Francesca. P. A. Fabbiani

Prof. Dr. Werner F. Kuhs

Abteilung Kristallographie,

Fakultät für Geowissenschaften und Geographie

\section{Members of the examination board}

Reviewer: Dr. Francesca. P. A. Fabbiani

Second reviewer: Dr. Werner F. Kuhs

Further members of the examination board:

Dr. Ben Corry

Dr. Ricardo Mata

Dr. Heidrun Sowa

Dr. Kai Tittmann

Date of the examination: June $9^{\text {th }}, 2016$ 


Where the spirits fall

Where live beautiful lies

Where I'm outa control

And I just cannot die

S.G.B. 



\section{Acknowledgements}

After painstakingly writing and formatting the other 220 pages of this document, it feels good to finally come to a place where I can lay the words unconstrained by the rigorous formality of scientific writing, a discipline about which, I just realised, I know rather little. My awesome supervisor, Francesca Fabbiani - often referred as Boss, pronounced with a delicate whiff of Spanish accent-has done everything in her power to sculpt my writing into what can be found in this dissertation. I am immensely thankful to her as a supervisor, mentor, and friend. For letting my curiosity wander around, but never too far from the path of productivity. For having always a witty response to my illogical digressions. For believing in this work even when I am still not completely sure of what I have accomplished.

I shall show my gratitude towards the members of my department, under the careful watch of Prof. Werner F. Kuhs, those who still linger around and those whose paths have diverged far from here. Sofiane, who has patiently answered all my questions, no matter how foreign. Stefano, who lifted the spirits during the time he spent freezing around here. Julian, always ready to discuss refinement strategies and willing to go through nightmarish paperwork. Patrick, from whom I have learnt almost everything I know about statistical mechanics - nothing like some hefty equations to deter people from peering at my desk - and a consid- 
erable bunch of approaches to computer simulations. Andrzej, who, now I come to realise, has been answering the same question about hydrate clathrates for the last three years, slightly rephrased every time. Lars, because being able to ask directly the person who built the diffractometer has proven a more efficient learning technique than trial and error. And Dr. Heidrun Sowa, whose input on space-group symmetry has saved a considerable amount of time in several occasions. I do not forget the technical staff who keeps this department running: Heiner, Jan Martin, Karin, Klaus, Nils, and Ulf. Thank you all.

I have also enjoyed the fabulous opportunity to jump around in Australian soil, as Francesca reckoned it was best to send me over $16000 \mathrm{~km}$ away if I were to learn about computer simulations, and Ben Corry gladly accepted the proposal and let me consume his computing time. I could not be more grateful to both for my time there; also to Natalie and Nishank, from whom I learned a fair amount.

I want to finish by mentioning a few friends, who have not contributed to the scientific dimension of this work, but without whom the results would have been different. Almudena, Jovana, and Aldo, thanks for you support, for-how to put it diplomatically - putting up with my utter lack of diplomacy. Claudia (Wandkte), my go-to girl to find things around the university. And the honourable mentions go to Claudia (Millán), who is yet to miss her usual summer in German lands, and to Sergio, who has earnestly kept up with the philosophical disquisition from the other side of the Internet since I changed the neck-wrecking winds of my hometown by the stiff coldness of the Northern regions. Thanks. 
This work has been made possible by a DFG grant (FA 964/1-1), awarded to Francesca Fabbiani, for which I am grateful. I would also like to acknowledge the beamtime granted at DORIS and PETRAIII (DESY) and the great work of the professionals working there, as well as the computing time granted at RAIJIN (NCI, at the Australian National University) and AVOCA (VLSCI, at the University of Melbourne). 



\section{Contents}

$\begin{array}{ll}\text { Introduction } & 1\end{array}$

References .................. 6

1 Experimental and theoretical background 9

1.1 High-pressure crystallography . . . . . . . . . . . . . . . 9

1.1.1 Diamond anvil cells . . . . . . . . . . . . 9 9

1.1.2 Experiments in a diamond anvil cell . . . . . . . 13

1.1.3 X-ray data collection and processing in a diamond anvil cell . . . . . . . . . . . . . . 14

1.2 Molecular dynamics simulations . . . . . . . . . . . 18

1.2.1 Equilibration fundamentals . . . . . . . . . 18

1.2.2 Trajectory analysis . . . . . . . . . . . . 24

1.2.3 Energy calculations . . . . . . . . . . . . . 24

1.2.4 Addressing the validity of molecular dynamics simulations . . . . . . . . . . . . 33

References . . . . . . . . . . . . . 35 
2 Formation of hydrates at high pressure

2.1 Introduction . . . . . . . . . . . . . . . . 39

2.2 Experimental . . . . . . . . . . . . . . . . 42

2.2.1 List of structures determined at high pressure . . . 42

2.2.2 Compressibility of lattice water . . . . . . . . 44

2.2.3 Pressure-volume diagrams of anhydrate-hydrate sets . . . . . . . . . . . . 4 46

2.3 Results and discussion . . . . . . . . . . . . . . 47

2.3.1 Compressibility of lattice water . . . . . . . 47

2.3.2 Assessment of errors . . . . . . . . . . . . . . . 48

2.3.3 Pressure-volume diagrams of anhydrate-hydrate sets . . . . . . . . . . . . . 51

2.4 Conclusions and outlook . . . . . . . . . . . . . 55

References . . . . . . . . . . . . . 58

3 Hydrates of $\beta$-cyclodextrin at high pressure 61

3.1 Introduction . . . . . . . . . . . . . . . 61

3.2 Experimental . . . . . . . . . . . . . . . . 64

3.2.1 Crystal growth . . . . . . . . . . . . . . 64

3.2.2 X-ray data collection and processing . . . . . . 66

3.2 .3 Structure refinement . . . . . . . . . . . . 67

3.3 Results and discussion . . . . . . . . . . . . 70

3.3.1 Twinning in $\beta$-cyclodextrin hydrate form II . . . . 70

3.3.2 Description of the structure of $\beta$-cyclodextrin form II . . . . . . . . . . . . . . . . 72

3.3.3 Description of the structure of $\beta$-cyclodextrin form III . . . . . . . . . . . . . . . 76

3.3.4 Hydration numbers of forms II and III of $\beta$-cyclodextrin . . . . . . . . . 78

3.4 Conclusions and outlook . . . . . . . . . . . . . . 83

References . . . . . . . . . . . . . . . 85

$4 \beta$-Cyclodextrin dimethylformamide 12.5 hydrate inclusion complex $\quad 89$

4.1 Introduction . . . . . . . . . . . . . 89 
4.2 Experimental . . . . . . . . . . . . . . . . . 90

4.2 .1 Crystal growth . . . . . . . . . . . . 90

4.2 .2 X-ray diffraction analysis . . . . . . . . . . 90

4.2 .3 XPAC analysis . . . . . . . . . . . . . . 91

4.3 Results and discussion . . . . . . . . . . . . . . 93

4.3.1 Structure description . . . . . . . . . . . . . 93

4.3 .2 Structure comparison . . . . . . . . . . . 95

4.4 Conclusions and outlook . . . . . . . . . . . . . 100

References . . . . . . . . . . . . . . . . 102

5 Hydration of $\alpha$-cyclodextrin at high pressure 105

5.1 Introduction . . . . . . . . . . . . . . . . 105

5.2 Experimental . . . . . . . . . . . . . . . . . 108

5.2.1 Crystal growth and compression study . . . . . . 108

5.2.2 X-ray data collection and processing . . . . . . . . 109

5.2.3 Structure refinement of $\alpha$-cyclodextrin hydrate at $0.2 \mathrm{GPa} \ldots \ldots \ldots \ldots \ldots$

5.2 .4 Molecular dynamics . . . . . . . . . . . . . . . 111

5.2.4.1 Equilibrations of infinite crystals . . . . . 111

5.2.4.2 Equilibrations of molecular clusters

in water . . . . . . . . . . . . . . . 114

5.2.4.3 Umbrella sampling . . . . . . . . . . . . 116

5.2.4.4 Metadynamics . . . . . . . . . . . . 118

5.3 Results and discussion . . . . . . . . . . . . . 120

5.3.1 Crystallographic evidence for hydration . . . . . 120

5.3.2 Hydration mechanism by molecular dynamics . . . 123

5.3.2.1 Equilibrations of infinite crystals . . . . . 124

5.3.2.2 Equilibrations of molecular clusters

in water . . . . . . . . . . . . . . 124

5.3.2.3 Umbrella sampling . . . . . . . . . . . . 126

5.3.2.4 Metadynamics . . . . . . . . . . . . . 127

5.3.3 High-pressure hydration mechanism . . . . . . . 130

5.4 Conclusions and outlook . . . . . . . . . . . . . . 132

References . . . . . . . . . . . . . . . 134 
6 High pressure study of the water-tert-butylamine system

6.1 Introduction . . . . . . . . . . . . . . . . 137

6.2 Experimental . . . . . . . . . . . . . . . . 138

6.2.1 Screening overview . . . . . . . . . . . . 138

$6.2 .2 \quad 5.65$-Hydrate . . . . . . . . . . . . . . 140

6.2.2.1 Crystal growth . . . . . . . . . . . 140

6.2.2.2 X-ray data collection and processing . . . 140

6.2.2.3 Structure refinement . . . . . . . . . . . . 140

$6.2 .3 \quad 5.8$-Hydrate . . . . . . . . . . . . . . . . 141

6.2.3.1 Crystal growth . . . . . . . . . . . 141

6.2.3.2 X-ray data collection and processing . . . 141

6.2.3.3 Structure refinement . . . . . . . . . . . . 142

6.2.4 Pure tert-butylamine form II . . . . . . . . . . . 142

6.2.4.1 Crystal growth . . . . . . . . . . . 142

6.2.4.2 X-ray data collection and processing . . . 144

6.2.4.3 Structure refinement . . . . . . . . . . . . 144

6.2.4.4 Molecular dynamics . . . . . . . . . . . 145

6.2.5 tert-Butylammonium carbonate . . . . . . . . . 149

6.2.5.1 Crystal growth . . . . . . . . . . . . 149

6.2.5.2 X-ray data collection and processing . . . 149

6.2.5.3 Structure refinement . . . . . . . . . . 149

6.3 Results and discussion . . . . . . . . . . . . . . . . . 149

6.3.1 Structure description . . . . . . . . . . . . . . . . 149

6.3.1.1 5.65-hydrate . . . . . . . . . 150

6.3.1.2 5.8-hydrate . . . . . . . . . 151

6.3.1.3 Pure tert-butylamine form II . . . . . . . 154

6.3.1.4 tert-Butylammonium carbonate . . . . 155

6.3.2 Crystalline phases in the water-tert-butylamine system . . . . . . . . . . . . . . 157

6.3.2.1 Density normalisation . . . . . . . . 159

6.3.3 Computational interpretation of pure tert-butylamine form II . . . . . . . . . . . 161

6.4 Conclusions and outlook . . . . . . . . . . . . . . . 164 
References . . . . . . . . . . . . . 167

$\begin{array}{ll}\text { Appendices } & 172\end{array}$

A List of structural sets . . . . . . . . . . . . . . . . 172

B Compressibility of lattice water . . . . . . . . . . 181

C Volume-pressure diagrams . . . . . . . . . . . . . . . 184

D List of available determinations of $\beta$-cyclodextrin form I . 191

E List of all the structures included in the XPAC calculation . . . . . . . . . . . . . . . . . . 192

F Detailed description of the disorder modelled in the high-pressure setup of $\alpha$-cyclodextrin used in the equilibration as an infinite crystal . . . . . . . . . . . 193

G Detailed description of the disorder modelled in the high-pressure setup of $\alpha$-cyclodextrin used in the equilibration as a finite crystal surrounded by water . . . 194

$\mathrm{H} \quad$ Complete results from the simulations of $\alpha$-cyclodextrin infinite crystals . . . . . . . . . . . . . . 196

I Technical description of the diamond anvil cells . . . . . 200

I.1 Square cell . . . . . . . . . . . . . . . . 200

I.2 Triangular cell . . . . . . . . . . . . . . . 201

I.3 $90 / 100^{\circ}$ cell . . . . . . . . . . . . . 202

I.3.1 Optimising data collection with the $90 / 100^{\circ}$ cell . . . . . . . . . . 202 



\section{Abbreviations and symbols}

$\alpha, \beta, \gamma \quad$ unit-cell angles

$\bar{\alpha}$ average thermal expansion coefficient

$\beta$ compressibility, or reciprocal thermodynamic temperature

$\epsilon \quad$ Lennard-Jonnes depth well

$\epsilon_{0}$ electrical permittivity

$\zeta$ Nosé-Hoover thermostat friction

$\theta$ deviation from ideal conformation, or diffraction angle

$\lambda$ trajectory coordinate, or wavelength

$\mu$ average, or chemical potential

$\nu \quad$ state of a system

$\rho$ crystal density, or electron density

$\sigma \quad$ Lennard-Jones equilibrium distance, or standard deviation

$\chi \quad$ order parameter

$\Omega \quad N V E$ partition function

$A$ Helmholtz free energy

ADP atomic displacement parameter

AP ambient-pressure

$a, b, c \quad$ unit-cell axes

$B_{0}$ bulk modulus at ambient pressure

$B_{0}^{\prime} \quad$ pressure derivative of the bulk modulus
CD cyclodextrin

$\alpha$-CD $\quad \alpha$-cyclodextrin

$\beta$-CD $\beta$-cyclodextrin

Corr correlation

Cov covariance

CSD Cambridge Structural Database

CSP crystal structure prediction

$C_{V} \quad$ constant-volume heat capacity

dabcoHI 1,4-diazabicyclo[2.2.2] octane hydroiodide

DAC diamond anvil cell

dMBzIm 5,6-dimethylbenzimidazole

$e$ electron

E energy

$f$ correction factor

$F$ force, or structure factor

FES (Gibbs) free energy surface

FOM figure of merit

$G \quad$ Gibbs free energy

$H$ enthalpy

HP high-pressure

$\mathrm{H}$ bond hydrogen bond

$h, k, l \quad$ Miller indices

$I \quad$ intensity of a reflection

$k_{\mathrm{B}} \quad$ Boltzmann constant

$K \quad$ kinetic energy 


\begin{tabular}{|c|c|}
\hline$L$ & length of a simulation box \\
\hline$m$ & mass \\
\hline$M$ & number of ideal particles \\
\hline MD & molecular dynamics \\
\hline$M$ & Parrinello-Rahman barostat coupling matrix \\
\hline$N$ & hydration number, or \\
\hline & number of particles \\
\hline$N P T$ & constant $N, P$, and $T$ \\
\hline NVE & constant $N, V$, and $E$ \\
\hline$N V T$ & constant $N, V$, and $T$ \\
\hline$P$ & pressure, or \\
\hline & probability \\
\hline $\mathrm{PBC}$ & periodic boundary conditions \\
\hline PME & particle mesh Ewald \\
\hline PTM & pressure transmitting medium \\
\hline$q$ & atomic charge \\
\hline$Q$ & Cremer-Pople puckering amplitude, or \\
\hline & mass of coupling bath, or \\
\hline & $N V T$ partition function \\
\hline$r$ & radius \\
\hline$R$ & $R$ factor \\
\hline RMS & root mean square \\
\hline RSS & residual sum of squares \\
\hline$s$ & reduced coordinate \\
\hline
\end{tabular}

$S$ entropy,or goodness of fit

$t$ time

$T$ temperature, or

transmission factor

${ }^{t} \mathrm{BA}$ tert-butylamine

$U$ potential

$U_{\mathrm{C}} \quad$ Coulomb potential

$U_{\text {LJ }} \quad$ Lennard-Jones potential

$v$ velocity

$V \quad$ unit-cell volume, or volume

$V_{\text {excl }}$ volume per chemical formula excluding water

$V_{H} \quad$ volume within a Hirshfeld surface

$V_{H, n t t} \quad$ unattributed volume per chemical formula

$V_{H, n w a t}$ volume of a molecule other than water

$V_{H, w a t}$ volume of a water molecule

$V_{M}$ molecular volume

$V_{Z} \quad$ volume per chemical formula

Var variance

$x, y, z \quad$ coordinates

Z NPT partition function, or number of molecules per unit cell 




\section{Introduction}

Molecular organic materials are ordered solids whose stability and structure is dictated by the intermolecular interactions of their constituent organic molecules. ${ }^{1}$ These materials are at the centre of many technological applications - from electronics (organic semiconductors) and photonics (organic lasers) to medicine (targeted drug delivery) - thanks to the fact that individual molecules can be functionalised and their arrangements tailored to obtain specific properties, giving rise to the field of molecular engineering. Intermolecular interactions are a key concept in this field, and a better understanding of these is essential to improve crystal designing techniques.

Polymorphism is both a blessing and a curse for crystal engineers. ${ }^{2}$ A blessing because different polymorphs might show different properties, proving that the final properties of a solid are not solely determined by its constituents, but also by their arrangement in the solid state. A curse because predicting which of the polymorphs found in a crystal's structural landscape - for instance, by means of crystal structure prediction (CSP) — will actually crystallise under certain experimental conditions, is not an easy task. The same can be said about solvate formation. 
Structural studies are a basic tool to broaden our understanding about intermolecular interactions, and provide very valuable information that can potentially be used to determine which crystalline phase will form and which properties it will have. Screening of crystalline phases of organic substances has been predominantly performed by means of temperature- and solvent-based techniques. However, the use of pressure, thanks to the development of the diamond anvil cell (DAC), has been attracting increasingly more interest. The past 15 years have seen an important rise in the number of studies of molecular materials by means of pressure. Compression and in-situ high-pressure crystallisation have proven a successful way to access phases (polymorphs and solvates, especially hydrates) which cannot be obtained by other means (see $\$ 1.1$ for a brief review on the topic).

Reporting new phases of molecular organic materials is of great interest to expand our knowledge; recently the interest has been turning towards understanding why these phases actually form. The work of Parsons, ${ }^{3-5}$ combining high-pressure crystallography with energy calculations performed with the PIXEL method, ${ }^{6}$ is probably the best example. The advantage of the PIXEL method is that it breaks down the energy of intermolecular interactions in four contributions: Coulombic, polarisation, dispersion, and repulsion. Upon compression, a phase transition might occur when, for one or more interactions, the repulsion outweighs the other three contributions as pressure brings the molecules closer together. In such a case, the phase transition is a way to release the internal strain of the structure. ${ }^{3}$ In other cases, the overall energy of the structure increases because of the $P V$ work; a phase transition towards a denser phase can stabilise the structure by decreasing the energy associated to the $P V$ term via smaller volumes. ${ }^{4}$ In both situations, the new phase is more efficiently packed due to less volume being available at higher pressures. In some cases, zero-point energies and entropic contributions can become important to lower the overall energy of the high-pressure favoured phase. ${ }^{5}$ Nevertheless, two polymorphs are related by a phase transition only if there is a suitable path for it. ${ }^{7}$ Certain phases cannot be accessed by direct compression, because the transformation is kinet- 
ically hindered, but can be accessed by in-situ crystallisation at high pressure. These studies benefit from the fact that pressure modifies the volume of a sample while keeping its thermal energy constant, which allows to directly correlate the changes observed in the sample with the applied pressure, something that is more complex when triggering crystal transformations by means of changes in temperature. ${ }^{8}$

The present work contains a collection of high-pressure crystallographic experiments combined with molecular dynamics (MD) simulations aimed at exploring how, rather than why, pressure modifies structures at the molecular level and molecular transformations can occur. Three organic materials have been studied: two cyclodextrins and tertbutylamine. While all these substances are interesting for their industrial applications, they have been selected as representatives of very different types of systems; the former are large molecules with a great ability to form hydrogen bonds (H-bond), while the latter is small and compact with limited capability of generating inter-molecular interactions. Studying such distinct systems allows to deepen our understanding of the behaviour of water in very different chemical environments. This is brought to light in the first chapter, where a database study including many different molecular hydrates is carried out and the differences between the substances are analysed.

\section{Database study on hydrate formation at high pressure}

Water is the most common solvent present in molecular organic materials,${ }^{9}$ and is easily incorporated in their structure due to its small size and H-bond forming capabilities. ${ }^{10}$ Despite its ubiquitous presence and a considerable amount of research on the topic (Ch. 2), our understanding of the role of water in organic crystals is still limited, and prediction and control of hydrate formation remains an open question in crystal engineering. ${ }^{11}$ A complete database study of hydrates formed at high pressure has been performed to bring to light trends on hydrate formation which can further contribute to systematise our overall knowledge on phase transformations. An equation of state for the compressibility of lattice water has been modelled as part of this study, providing an use- 
ful tool to easily compare hydrates with their corresponding anhydrous forms at pressures below $5 \mathrm{GPa}$.

\section{Cyclodextrins}

Cyclodextrins (CDs) are cyclic derivatives of starch formed by several $\alpha$-D-glucopyranose units linked together by $(1 \rightarrow 4)$ O-glycosidic bonds. Research on this type of compounds is mainly focused on those comprising six $(\alpha$-CD), seven $(\beta-C D)$ and eight $(\gamma-\mathrm{CD})$ pyranose units. Both $\alpha$ and $\beta$-CD have been studied in this work (Ch. 5 and Chs. 3 and 4 , respectively). Topologically, CDs are described as hollow truncated cones in which the wider opening of the cavity is delimited by the secondary hydroxyl groups. The inner part of the cone is mainly apolar because of the alkyl groups of the glucose rings, while the outer part is polar thanks to the presence of several hydroxyl groups. ${ }^{12}$

Interest on CDs arises from their ability to form inclusion compounds with guests that fit in the cavity and have some capability of H-bond formation. ${ }^{13,14}$ Thanks to this property, CDs have found a vast number of applications. To name a few, CDs are used as agents to improve solubility, stability, and bioavailability of pharmaceutical substances, ${ }^{15-18}$ as molecular reactors, ${ }^{19-23}$ in the constructions of molecular machines, ${ }^{24-26}$ and as enzime models. ${ }^{27-32}$

In the framework of this work, CDs provide a challenge due to their large size and difficulty to crystallise in-situ at high pressure, but also a helpful insight in the high-pressure behaviour of large systems, especially in relation to their water content. In the case of $\alpha$-CD, application of pressure does not promote a phase transition; instead, the structure undergoes a conformational change in order to increase its water content and reduce the overall volume of the system. The mechanism of this process has been explored via MD simulations. $\beta$-CD shows a very different behaviour, and two different super-hydrated phases have been obtained at high pressure. The formation of these phases is discussed in relation to the overall behaviour of hydrate formation discussed in Ch. 2 . 


\section{tert-Butylamine}

tert-Butylamine $\left({ }^{t} \mathrm{BA}\right)$ is a very small molecule and exhibits a completely different behaviour than CDs in terms of hydrate formation, as it forms semi-clathrates. Water inclusion compounds are crystalline solids consisting of a guest (a small molecule) trapped within water cages, formed by H-bonded water molecules. ${ }^{33}$ Water inclusion compounds can be classified into three different groups: gas hydrates, alkylamine hydrates, and quaternary ammonium salt hydrates. ${ }^{34}$ Only gas hydrates are true clathrates, and as such their water cages are characterised by faces in the shape of regular polyhedra. The other two categories are termed semi-clathrates because, although their structure generally resembles that of a true clathrate, they exhibit interactions between the guest molecule and the host water, which causes the shape of the cages to deviate from regular polyhedra. Clathrates hydrates, particularly gas hydrates, are relevant as gas storage materials, appearing naturally in the seafloor, and due to the problems they originate in production pipelines. ${ }^{33}$

Together with the two structures presented in this work, a total of nine different crystalline ${ }^{t} \mathrm{BA}$ hydrates have been reported. Although semi-clathrates are structurally closer to ice than to the organic hydrates presented in Ch. 2, and a comparison with the latter seems inappropriate, a system with so many different hydrates is a perfect example of how the use of high-pressure crystallisation provides access to the densest phases in the system. The study on ${ }^{t} \mathrm{BA}$ hydrates reveals that a thorough screen of hydration capabilities necesitates the exploration of pressure space, which can help rationalising trends observed at ambient pressure.

As part of this study a new structure of pure ${ }^{t} \mathrm{BA}$, which can crystallise as two different polymorphs depending on the pressure, has also been reported. The high-pressure phase has been also studied by MD to better understand its behaviour, and on the basis of the simulation results it has been classified as an orientational glass. ${ }^{35,36}$ 


\section{References}

[1] A. I. Kitaigorodskii, Molecular Crystals and Molecules, Academic Press, 1973.

[2] D. Braga, L. Brammer and N. R. Champness, CrystEngComm, 2005, 7, 1-19.

[3] P. A. Wood, R. S. Forgan, D. Henderson, S. Parsons, E. Pidcock, P. A. Tasker and J. E. Warren, Acta Cryst., 2006, B62, 1099-1111.

[4] R. D. L. Johnstone, D. Francis, A. R. Lennie, W. G. Marshall, S. A. Moggach, S. Parsons, E. Pidcock and J. E. Warren, CrystEngComm, 2008, 10, 1758-1769.

[5] R. D. L. Johnstone, A. R. Lennie, S. F. Parker, S. Parsons, E. Pidcock, P. R. Richardson, J. E. Warren and P. A. Wood, CrystEngComm, 2010, 12, 1065-1078.

[6] A. Gavezzotti, J. Phys. Chem. A, 2003, 107, 2344-2353.

[7] E. Boldyreva, Cryst. Growth Des., 2007, 7, 1662-1668.

[8] R. Winter and J. Jonas, High-Pressure Chemistry, Biochemistry and Materials Science, Springer Science \& Bussiness Media, 1993.

[9] C. H. Görbitz and H.-P. Hersleth, Acta Cryst., 2000, B56, 526-534.

[10] A. L. Gillon, N. Feeder, R. J. Davey and R. Storey, Cryst. Growth Des., 2003, 3, 663-673.

[11] H. D. Clarke, K. K. Arora, H. Bass, P. Kavuru, T. T. Ong, T. Pujari, L. Wojtas and M. J. Zaworotko, Cryst. Growth Des., 2010, 10, $2152-2167$.

[12] G. Wenz, Angew. Chem. Int. Ed., 1994, 33, 803-822.

[13] W. Saenger, Angew. Chem. Int. Ed., 1980, 19, 344-362.

[14] K. K. Chacko and W. Saenger, J. Am. Chem. Soc., 1981, 103, $1708-1715$.

[15] F. Castelli, G. Puglisi, G. Giammona and C. A. Ventura, Int. J. Pharm., 1992, 88, 1-8.

[16] A. Paulidou, D. Maffeo, K. Yannakopoulou and I. M. Mavridis, CrystEngComm, 2010, 12, 517-525.

[17] K. T. Arun, D. T. Jayaram, R. R. Avirah and D. Ramaiah, J. Phys. Chem. B, 2011, 115, 7122-7128. 
[18] S. M. Bruno, J. A. Fernandes, J. Marques, S. C. Neto, P. J. RibeiroClaro, M. Pillinger, F. A. Almeida Paz, M. P. M. Marques, S. S. Braga and I. S. Gonçalves, Eur. J. Inorg. Chem., 2011, 4955-4963.

[19] K. Takahashi, Chem. Rev., 1998, 98, 2013-2033.

[20] T. J. Brett, J. M. Alexander, J. L. Clark, C. R. Ross II, G. S. Harbison and J. J. Stezowski, Chem. Commun., 1999, 1275-1276.

[21] T. J. Brett, J. M. Alexander and J. J. Stezowski, J. Chem. Soc., Perkin Trans. 2, 2000, 1105-1111.

[22] Y. Nishioka, T. Yamaguchi, M. Yoshizawa and M. Fujita, J. Am. Chem. Soc., 2007, 129, 7000-7001.

[23] Z. Yang and H. Ji, ACS Sustainable Chem. Eng., 2013, 1, 11721179 .

[24] A. Harada, Acc. Chem. Res., 2001, 34, 456-464.

[25] S. A. Nepogodiev and J. F. Stoddart, Chem. Rev., 1998, 98, 19591976.

[26] X.-J. Shen, H.-L. Chen, F. Yu, Y.-C. Zhang, X.-H. Yang and Y.-Z. Li, Tetrahedron Lett., 2004, 45, 6813-6817.

[27] R. Breslow and S. D. Dong, Chem. Rev., 1998, 98, 1997-2012.

[28] N. M. Milović, J. D. Badjić and N. M. Kostić, J. Am. Chem. Soc., 2004, 126, 696-697.

[29] F. Ortega-Caballero, C. Rousseau, B. Christensen, T. E. Petersen and M. Bols, J. Am. Chem. Soc., 2005, 127, 3238-3239.

[30] K. Kano, H. Kitagishi, C. Dagallier, M. Kodera, T. Matsuo, T. Hayashi, Y. Hisaeda and S. Hirota, Inorg. Chem., 2006, 45, 4448-4460.

[31] L. G. Marinescu and M. Bols, Angew. Chem. Int. Ed., 2006, 45, 4590-4593.

[32] M. L. Singleton, J. H. Reibenspies and M. Y. Darensbourg, J. Am. Chem. Soc., 2010, 132, 8870-8871.

[33] E. D. Sloan and C. A. Koh, Clathrate hydrates of natural gases, CRC Press, 3rd edn, 2007.

[34] G. A. Jeffrey, J. Inclusion Phenom., 1984, 1, 211-222.

[35] S. C. Abrahams and W. N. Lipscomb, Acta Cryst., 1952, 5, 93-99. 
[36] J. C. W. Folmer, R. L. Withers, T. R. Welberry and J. D. Martin, Phys. Rev. B, 2008, 77, 1-10. 


\section{Experimental and theoretical background}

\subsection{High-pressure crystallography}

\subsubsection{Diamond anvil cells}

The first design of a DAC was reported in 1959 by Weir et al., ${ }^{1}$ based on the previous design of an opposed anvil press by Bridgman. ${ }^{2}$ The major breakthrough of the DAC was the use of diamonds as anvils, which not only allowed to reach very high pressures, but also made it possible to visually inspect the samples during pressure application, thanks to the transparent windows. This first design became very popular, as measurement techniques such as microscopy, IR spectroscopy, or X-ray diffraction could be applied to compressed samples. ${ }^{3}$

DACs evolved over fifteen years until Merrill and Bassett presented their own design, ${ }^{4}$ later improved by Hazen and Finger, ${ }^{5}$ on which most modern cells are based (Fig. 1.1). This type of cell, which is small enough 


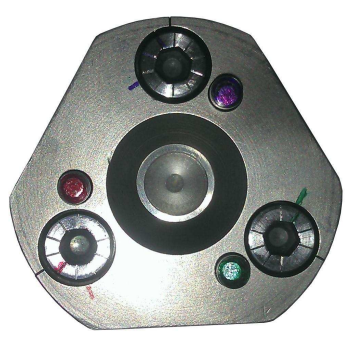

Figure 1.1: DAC based on the design by Merrill and Bassett.

to fit in the palm of a hand and can be easily mounted on a conventional diffractometer and many other instruments, has been at the core of high-pressure research since its creation. ${ }^{6}$ In the Merrill-Bassett design, each diamond is supported on a backing plate, which in turn is mounted on a metal body. At the beginning, diamonds used as anvils had the brilliant cut, or a modification of this, which is characterised by a large flat table - the top part of the diamond (Fig. 1.2) - that is directly supported on the baking plate. In order to offer enough support, this design requires backing plates with relatively narrow window openings. Experimental results benefited enormously from the introduction of the Boehler-Almax diamond cut, ${ }^{7}$ which is supported on the crown rather than on the table (Fig. 1.2), hence enough support can be provided even with wider window openings $\left(\sim 90^{\circ}\right)$ and higher pressures can be attained. ${ }^{8}$ Ahsbahs introduced another type of cut in which the table is not flat but approximately spherical, which adds to the previous advantages the fact that the optic path trough the diamond is equally long in all orientations of the DAC, removing the need to correct for the absorption of the diamonds. ${ }^{9}$ In both cases, the support also attempts to facilitate the alignment of the diamonds.

Proper placement of the diamonds on the backing plates and alignment of the diamonds relative to each other is critical to ensure a homogeneous distribution of pressure and to minimise the risk of gasket failure, which can lead to diamond fracture. A modification of the alignment system was key to increase the pressure range of the DAC up to $25 \mathrm{GPa} .{ }^{10}$ Backing plates were originally made out of beryllium; its transparency to X-rays was advantageous when only narrow windows were available. Steel or tungsten carbide baking plates, which block $\mathrm{X}$-ray radiation, in combination with different wide window geometries, are preferred nowadays. ${ }^{9,11}$ Synthetic diamond backing plates, which allow to reach pressures up to $223 \mathrm{GPa}$, are also available. ${ }^{12}$

The sample chamber is formed by a drilled gasket which is placed in between the two diamonds, forming a sealed compartment when pressure is applied (Fig. 1.2). Inside the chamber there should be a pressure calibrant, a pressure transmitting medium (PTM), and the sample itself 


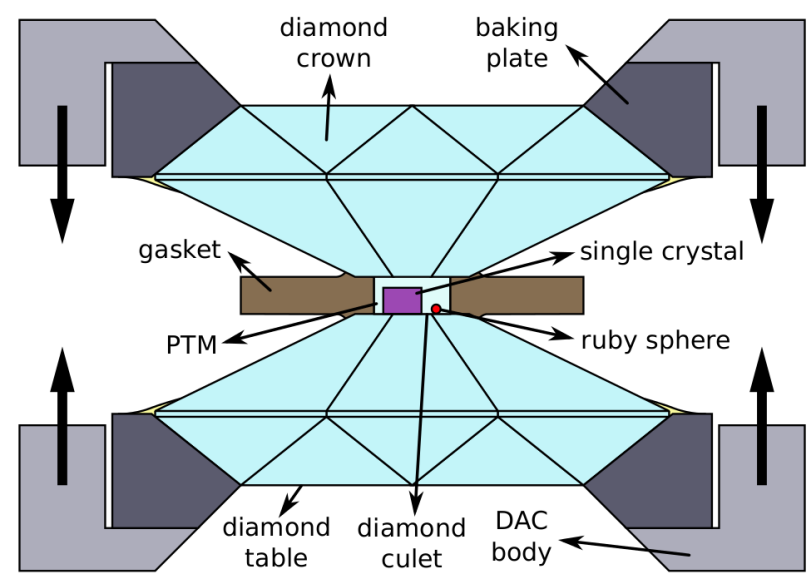

(liquid samples and samples in solution can act as PTM on their own), as described in the following section. A variety of gasket materials are available, and in this work Inconel 718, steel, and brass are used, with thickness around $150 \mu \mathrm{m}$, pre-indented according to the guidelines from Dunstan, ${ }^{13}$ depending on each specific DAC used. In all the DACs employed in this work (Appx. I), pressure is applied by means of screws connecting the two steel bodies which house the diamonds on their backing plates. The load is transmitted from a relatively large surface on the crown of the diamonds to a much smaller one on the culet, with the subsequent amplification. Load transmission can be further controlled by the volume of the sample chamber (diameter of the hole and thickness of the gasket); for the same position of the pressing screws, the smaller the sample chamber the higher the pressure. Pressure is applied by the diamonds to the PTM and also to the gasket, which through plastic deformation allows to recreate hydrostatic conditions in the interior of the sample chamber, up to the solidification point of the PTM $(\sim 1 \mathrm{GPa}$ in the case of aqueous solutions). As most of the work here presented is limited to low pressures, below the formation of ice VI, relatively large sample chambers are used whenever possible, with the intention to grow larger crystals to facilitate the collection of X-ray data. Pressure inside the sample chamber is monitored by the shift of the $R_{1}$ fluorescence line of $\mathrm{Cr}^{3+}$, from a small ruby sphere loaded along with the sample. ${ }^{14,15}$ For all the experiments here reported, a $532 \mathrm{~nm}$ laser was used as an excitation source and the shift was measured on an OceanOptics USB4000
Figure 1.2: Diagram (not to scale) of a DAC with Boehler-Almax diamonds. The thick arrows indicate the direction in which pressure is applied via the pressing screws. 
spectrometer (the uncertainty of pressure measured by this method is $0.05 \mathrm{GPa}$ ). For further information on the design and operation of the DAC, refer to the work of Miletich et al. ${ }^{16}$ For a technical description of the DACs used in this work, see Appx. I.

The development of the DAC has not been idle in the recent years, but has steered towards the increase of the range of applications of the device. A DAC especially designed to work at the lower end of the pressure scale, with improved viewing windows, has been designed and used to monitor (by means of microscopy and spectroscopy) the behaviour of microbes under pressure. ${ }^{17}$ At this range of pressures, calibration with the ruby method is not sensitive enough, and a fluorescent dye embedded in a polymeric matrix is used instead. Such sensors can be used in aqueous solution up to $323 \mathrm{~K}$ in the $3-12 \mathrm{pH}$ range. For the purpose of magnetic measurements at cryogenic temperatures, pressure cells constructed of metal are impractical, as the magnetic field will induce current in the metallic parts, heating the sample. A plastic DAC has been built for such studies, substituting the gasket by a polymer containing diamond powder. ${ }^{18}$ The pressing screws have been substituted by a turnbuckle mechanism, which allows for further miniaturisation. A similar turnbuckle pressing mechanism has been implemented in metal to create a miniature DAC which can be fully immersed in a conventional cryostream of a diffractometer, allowing for easy low-temperature high-pressure diffraction studies. ${ }^{19}$ In neutron diffraction, the Paris-Edinburgh cell ${ }^{20}$ has been the preferred choice for high-pressure, because of its larger sample chamber, although recently Binns et al. reported being successful on the use of a miniaturised Merril-Bassett DAC constructed of a copper-beryllium alloy for the collection of neutron diffraction data. ${ }^{21}$ Further development of DACs is oriented towards creating wider windows while being able to attain very high pressures. A DAC with an opening angle of $100^{\circ}$ on one side and $90^{\circ}$ on the other, equipped with flat diamonds and the sample chamber drilled in polymer (supported on a traditional metallic gasket), has been used in this work. See Appx. I.3 for a more detailed description. 


\subsubsection{Experiments in a diamond anvil cell}

There are three distinct types of experiments that can be performed with DACs: in-situ crystallisation of liquids, in-situ crystallisations of solids from solution, and compression studies. ${ }^{22}$ The present work contains examples of all three cases.

In an in-situ crystallisation experiment, a crystal is directly grown under pressure inside the DAC. The simplest implementation consists of loading a liquid; however, the vast majority of molecular organic materials are solid at ambient pressure. Crystallisation of liquids and solids from solution are formally identical, as discussed below, although liquids have the advantage that the size of the crystals obtained is not a limitation, as the entire content of the sample chamber usually crystallises. For solid materials, a DAC is loaded with a saturated solution of the material in an appropriate solvent. The solution acts as the PTM, that is, the substance which receives the pressure from the diamonds and then transfers it to the crystal once it is formed. Under compression, high density phases are favoured; this promotes crystallisation, as solids tend to be denser than liquids and solutions. In the case of materials which can crystallise in different phases, either polymorphs, solvates, or cocrystals, denser phases are usually favoured during high-pressure crystallisation (see for instance the work by Fabbiani ${ }^{23-28}$ and Katrusiak $\left.^{29-31}\right)$. There are, nevertheless, exceptions to this behaviour, CDs being a good example (Chs. 3 and 5).

When crystallisation first occurs on increasing pressure, a polycrystalline sample is usually obtained, because at this stage the pressurisation rate tends to be fast and the sample is driven past the metastable zone for crystallisation. At this point, isobaric temperature cycling can be used to produce a single crystal. Cycling consists in heating to reduce the number of crystals in the sample chamber by dissolving them and then cooling to let the remaining crystals grow. The process is repeated until a single seed remains in the chamber, which is subsequently let to grow until it reaches a suitable size for X-ray diffraction. Once a single crystal is formed, pressure can be increased to ensure that the crystal will not further dissolve (for instance, under the heat of the X-ray 
beam) or to explore possible phase transitions upon compression. Although having a unique single crystal inside the DAC is desirable, with the availability of micro-focussed synchrotron beams it is now possible to collect data on a specific small crystal in the presence of others.

One of the main limitations of in-situ crystallisation is the solubility of the sample in the solvent used as PTM. The amount of solution that can be loaded in a DAC is below $0.02 \mathrm{~mm}^{3}$, which results in a very small amount of sample if said sample is poorly soluble, which does not allow to grow crystals of a suitable size for X-ray diffraction. To overcome this problem, crystals grown at ambient pressure can be loaded along with the saturated solution; subsequently the DAC is heated to dissolve the crystals in situ and effectively increase the concentration of the solution. In the particular case of CDs, heating is not needed, as the crystals dissolve, increasing the concentration of the solution, upon compression.

In a compression study, a crystalline sample is submitted to a gradual increase in pressure; data are collected at different pressure steps to identify and characterise possible phase transitions and changes at the molecular level (see for instance the work by Parsons, ${ }^{32-37}$ Boldyreva, ${ }^{38-40}$ and Lee ${ }^{41,42}$ ). X-ray diffraction and Raman spectroscopy are commonly used to analyse the compressed samples. To eliminate effects originating from the solvent, inert PTMs are usually employed (for instance, perfluorinated alkenes or pentane/cyclopentane mixtures in the case of polar substances). In other instances, an interacting PTM is desired, when it is the combination of an appropriate solvent and pressure which causes changes in the material under study, as it is the case of pressure-induced hydration (Ch. 5) and phase transitions that may be otherwise kinetically hindered. ${ }^{22}$

\subsubsection{X-ray data collection and processing in a diamond anvil cell}

High-pressure X-ray diffraction using a DAC suffers from three main problems: ${ }^{43}$ small size of the sample, reduced access to the reciprocal space, and reflections with untrue intensities. 
1.1. High-pressure crystallography

The small size of the sample can potentially be a problem, as indicated in the previous sections, but the availability of high-intensity micro-focused sources allows to measure small organic crystals in the home laboratory routinely. For the most difficult cases, or when rapid data collection is required, synchrotron sources offer a great alternative, with brighter beams which produce intense reflections even from poorly-diffracting crystals, and micro-focussed beams which allow to collect data on very small crystals, even in the presence of other specimens inside the sample chamber.

The access to the reciprocal space is limited by the body of the cell. ${ }^{16}$ Only reflections coming out of the sample at an angle smaller than the opening of the window of the DAC can be collected. This translates into a cone of reflections in direct space - two cones joined by their vertices when the data is collected in both sides of the DAC - or a torus in reciprocal space (Fig. 1.3). This is seen in the diffraction frames in the form of a shadow, a region which contains no reflections. That is, both completeness and redundancy are compromised. The problem is more noticeable for low symmetry crystals (triclinic, monoclinic, and orthorhombic), typical for organic materials. The situation can improve with a proper data collection strategy and the use of shorter X-ray wavelengths, which increases the effective size of the Ewald sphere, allowing to collect more reflections. The use of the DAC affects the final results of structure refinement, as statistics based on incomplete data are less reliable and a low data-to-parameter ratio limits the numbers of paramaters that can be refined. Moreover, systematic missing data results in the stretching of the electron density along the missing direction-perpendicular to the torus in reciprocal space - which can result for instance into a systematic error in the shape of the atomic displacement parameters.

In addition to the limited completeness, X-ray diffraction data collected at high pressures is affected by more absorption than for crystals collected in air (diamonds, gasket, baking plates, and PTM) and might show undesired diffraction from the diamonds and the gasket (and the baking plates if these are made out of beryllium). ${ }^{44,45}$ Although modern software has proven successful in performing absorption correction 
Figure 1.3: Cross section of a torus of reflections in the reciprocal space, characteristic of data collections of samples inside a DAC.

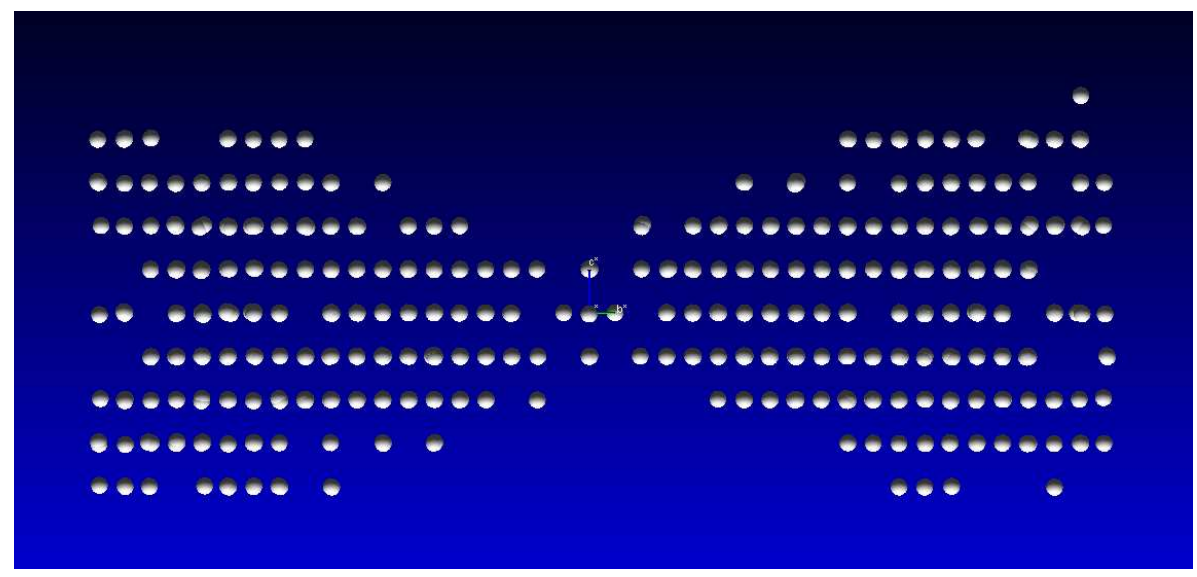

for high-pressure samples, it can be challenging considering the limited redundancy of the data. ${ }^{43}$ Repetition of the data collection is usually a good approach to improve redundancy, although it is time consuming and might not be possible if radiation damage is present.

Considering the difficulties outlined before, the data-collection strategy for single crystals contained in a DAC is oriented towards increasing completeness and redundancy. Usually, as much data as possible is collected, independently of the symmetry of the crystals. For the purpose of this work, the guidelines from Dawson et al. were followed. ${ }^{46}$ On a three-circle diffractometer, the maximum coverage of the reciprocal space, which primarily depends on the window opening of each DAC, is performed via $\omega$ scans, with additional $\phi$ scans added to increase redundancy. Each scan is actually double: first a scan is performed with the front of the DAC facing the incoming beam, and subsequently another one is performed with the back side facing the beam. In the absence of a fourth circle, the DAC is manually rotated on the goniometer head around the axis of the beam and the previous strategy is repeated for two or three different orientations. This significantly improves the redundancy of the data, but might introduce centring problems which need to be managed during the data reduction. In the case of diffractometers with only $\phi$ movement available, $\phi$ scans are performed, as wide as the window opening, on both sides of the DAC, combined with the aforementioned manual rotation. Shorter wavelengths and larger detectors are highly desirable in this configuration. 
Data processing is performed in a similar way than it would for crystals measured at ambient-pressure conditions, although some differences need to be addressed. ${ }^{46}$ As indicated previously, statistics tend to be less reliable when data completeness and redundancy is low. It is advisable in these cases to perform the complete integration, all needed corrections, and merging of different scans following different strategies, and use the end result of the refinement to asses which one of the strategies is the best approach.

Firstly, it should be considered whether different scans should be integrated together or separately. From the experience of this work, the latter tends to yield better results, due to the inherent difficulties of centring the DAC. ${ }^{47}$ SAINT $^{48}$ has been the integration software of choice for most samples, although CRYSALIS PRO ${ }^{49}$ has provided better results for samples collected using a Perkin-Elmer XRD 1621 amorphous-silicon flat panel detector, installed on the P02.2 beamline at the PETRAIII synchrotron (Ch. 3.2.2). Both programs include dedicated features to process high-pressure data, namely the ability to integrate only in the non-shadowed areas of the frames. The shadowed areas can be completely removed from the integration by specifying in the software the DAC opening angle. Specifying an angle smaller than the actual one eliminates reflections which suffer from partial shadowing, but this might negatively affect the total amount of integrated data. If the true opening angle is indicated in the integrations program, it is possible to use SHADE $^{50}$ after integration to filter problematic reflections. This program can also be used to remove partially-shadowed reflections or to remove reflections originating from the diamonds, which are sometimes integrated if overlapping with sample reflections. As part of this work a new version of this software, called SomBRA, has been written to apply similar corrections to data collected using DACs with asymmetric windows (Appx. I.3).

Secondly, absorption correction is critical to reduce errors on the measured intensities. In this work, absorption corrections have been performed with SADABS ${ }^{51}$ (or TwINABS ${ }^{52}$ for twinned samples). When each scan is integrated separately, the point at which all data are analysed 
together can affect the final result, as there is a different amount of data available to perform the absorption correction. Merging can be performed at the same time than absorption correction in SADABS, or afterwards, using a dedicated program, like SORTAV ${ }^{53}$ or XPREP ${ }^{54}$, or the refinement software, SHELXL ${ }^{55}$ in this case. All options have been routinely tried for all samples, selecting the procedure that yielded the best results in each case.

\subsection{Molecular dynamics simulations}

§1.2.1, §1.2.2, and §1.2.4 constitute a brief review about molecular dynamics, in the same way the previous section was a review about high-pressure crystallisation and crystallography. \$1.2.3 is aimed to demonstrate how energies can be computed from a simulated trajectory. Here I have used the teaching approach by P. G. Lafond, to whom I am grateful, which starting from the definition of energy works its way up demonstrating the partition functions of the NVE, NVT, and NPT ensembles. Subsequently, the relationship between the last two and the characteristic free energies of each ensemble-Helmholtz in NVT and Gibbs in NPT-are demonstrated. Finally, the variation of Gibbs free energy between two states in the NPT ensemble, based on their relative probabilities, is determined. Most of the equations in this section can be found in D. Frenkel and B. Smit, Understanding Molecular Simulation: From Algorithms to Applications, Academic Press, 2001, although the information has been rearranged according to the aforementioned scheme, and additional steps have been included and certain concepts have been simplified in the demonstrations for clarity. The raison d'êtrè of this section is to provide a more concise approach to energy calculation than that found in text books, with additional explanations to make it easier to read for those with limited knowledge in statistical mechanics, and more importantly, is a personal exercise to test my understanding of the field.

\subsubsection{Equilibration fundamentals}

MD is a computer simulation technique which, based on an appropriate force field describing the system under study, predicts the behaviour over time of such system by numerically solving the equations of motion. ${ }^{56}$ Differently from other simulation techniques, MD focusses on the time 
evolution of the system, a paramount factor when studying conformational changes in molecular systems. In MD, processes taking place at the molecular level are described by means of classical mechanics. The interaction between any two atoms in the system, separated a distance $r$, is described by a Lennard-Jones potential $\left(U_{\mathrm{LJ}}\right)$ :

$$
U_{\mathrm{LJ}}(r)=4 \epsilon\left[\left(\frac{\sigma}{r}\right)^{12}-\left(\frac{\sigma}{r}\right)^{6}\right]
$$

Where $\epsilon$ is the depth of the potential well and $\sigma$ is the distance at which the potential is zero. The atoms experience attraction when they are within range of each other due to Van der Waals or covalent interactions, but repulsion becomes dominant below a certain distance due to the overlap of their atomic orbitals. When the two atoms are separated further apart than the range of the Lennard-Jones potential, a Coloumb potential $\left(U_{\mathrm{C}}\right)$ is better suited to describe their interaction:

$$
U_{\mathrm{C}}(r)=\frac{1}{4} \epsilon_{0} \pi\left(\frac{q_{i} q_{j}}{r}\right)
$$

Where $\epsilon_{0}$ is the electrical permittivity of the medium and $q_{i}$ and $q_{j}$ are the charges of each atom. In a given system, each atom exerts a force $F(r)$ on any other atom according to the aforementioned potentials:

$$
F(r)=-\frac{\mathrm{d} U(r)}{\mathrm{d} r}=-\frac{\mathrm{d}}{\mathrm{d} r}\left[U_{\mathrm{LJ}}(r)+U_{\mathrm{C}}(r)\right]
$$

Once the forces are known, changes in the acceleration experienced by any atom due to interaction with other atoms can be determined from Newton's second law:

$$
\begin{gathered}
F(r)=m \frac{\mathrm{d} v}{\mathrm{~d} t}=m \frac{\mathrm{d}^{2} r}{\mathrm{~d} t^{2}} \quad \Rightarrow \\
\frac{\mathrm{d} v}{\mathrm{~d} t}=\frac{\mathrm{d}^{2} r}{\mathrm{~d} t^{2}}=\frac{F(r)}{m}
\end{gathered}
$$

Where $m$ is the mass of the atom, $r$ its position, $v$ its velocity, and $t$ is the time. Then, the trajectory of any atom, composed of its position and velocity over time, can be calculated.

The large number of atoms which usually conform molecular sys- 
tems makes it impossible to integrate the equations of motion exactly. Instead, numerical integration is used. Although there are different integration schemes, MD motors used in this work (NAMD ${ }^{57}$ and GROMOS ${ }^{58}$ ) employ variations of the Verlet scheme ${ }^{59}$ for its efficiency and numerical stability - that is, avoiding amplification of errors during the simulation.

Newton's laws of motion are defined for systems at constant energy, hence MD simulation are performed in the $N V E$ ensemble (constant number of particles, volume, and energy) by default. Structural changes in molecules rarely occur at constant energy. In order to perform simulations in the $N V T$ ensemble (at constant temperature), which is more useful from a chemical perspective, certain adjustments are needed. ${ }^{59,60}$

According to the equipartition theorem, the kinetic energy $(K)$ associated to each degree of freedom of a system is:

$$
K=\frac{1}{2} k_{\mathrm{B}} T
$$

Where $k_{\mathrm{B}}$ is the Boltzmann constant and $T$ is the temperature. For an atom, which can move in three directions:

$$
K=\frac{3}{2} k_{\mathrm{B}} T
$$

In a molecular system composed of $N$ atoms:

$$
\begin{gathered}
K=\frac{3 N}{2} k_{\mathrm{B}} T=\sum_{i=1}^{N} \frac{1}{2} m_{i} v_{i}^{2} \Rightarrow \\
T=\frac{1}{(3 N-3) k_{\mathrm{B}}} \sum_{i=1}^{N} m_{i} v_{i}^{2}
\end{gathered}
$$

Three degrees of freedom have been removed, as they correspond to the movement of all the atoms as a single entity, which is not considered in MD. In addition to Eq. 1.7, the velocities of the atoms in the system must behave according to a Maxwell-Boltzmann distribution:

$$
P(v) \propto \mathrm{e}^{-\beta K}
$$

Where $\beta$ is $1 /\left(k_{\mathrm{B}} T\right)$. 
In order to simulate a system in isothermal conditions, the velocities must be rescaled so that the temperature is in agreement with both Eqs. 1.7 and 1.8. This can be achieved in different ways. The simplest method consists in calculating the temperature after each step of the simulation, computing a scaling factor for the velocities in such a way that the temperature agrees with the aforementioned equations, and finally rescaling the velocities according to the scaling factor. While this method provides the right temperature, the velocity distribution is prone to errors. A better approach to reproduce the correct velocity distribution is the Andersen thermostat, which resets the velocities after a certain number of steps, taking new values directly from the Maxwell-Boltzmann distribution. This method has the disadvantage that non-realistic values for the velocities can be generated, which in turn generates non-physical trajectories. More realistic methods are based on the idea of coupling the simulated system with a bath. The Berendsen thermostat adds a heat bath which is at the target temperature and absorbs the temperature fluctuations of the system until both temperatures - of the system and the bath - are equilibrated. In reality this is not an isothermal process, and errors may arise for small systems, although the approximation is usually acceptable for larger systems. The method of choice in modern software is the Nosé-Hoover thermostat, which reformulates the equation of motion (Eq. 1.4):

$$
\frac{\mathrm{d} v}{\mathrm{~d} t}=\frac{F(r)}{m}-\zeta v
$$

Being:

$$
\frac{\mathrm{d} \zeta}{\mathrm{d} t}=\frac{1}{Q} \sum_{i}\left(m_{i} v_{i}^{2}-3 N k_{\mathrm{B}} T\right)
$$

Where $\zeta$ is the friction between the bath and the system and $Q$ is the mass of the bath. The thermostat works by sinking part of the energy of the system into the bath, until the temperatures of both equalise. This method provides the right temperature and distribution of velocities.

The simulations reported in this work have been performed in the NPT ensemble (constant pressure). Isobaric conditions are achieved by approaches similar to those explained above for isothermal processes. ${ }^{59,60}$ In the same way that the velocities - and hence the kinetic energy - can 
be rescaled to generate the correct temperature, the volume of the simulation box can be rescaled over time to generate the target pressure. Similarly to the temperature case, simply rescaling the simulation box does not necessarily generate the right pressure distribution. The preferred approached is the Parrinello-Rahman barostat, which is formally equivalent to the Nosé-Hoover thermostat:

$$
\frac{\mathrm{d} v}{\mathrm{~d} t}=\frac{F(r)}{m}-\boldsymbol{M} v
$$

Where $\boldsymbol{M}$ is a coupling matrix. The main advantage of this barostat is that pressure is treated as a stress tensor, allowing each dimension of the simulation box to change independently.

Another parameter to be addressed in MD simulations is the length of each time step. Numerical integration of the equations of motion works by determining the next point of the trajectory based on the immediately previous one, which generates accurate trajectories only when very short steps are used (0.1 or $0.2 \mathrm{fs}$ ). Such small time steps result in very long computation times, which are not practical for simulations of systems over thousands of atoms over hundreds of nanoseconds, as used here.

There are two well-known approaches to either allow for larger time steps or reduce the number of calculations in each step. ${ }^{60}$ In rigid dynamics, chemical bonds are considered to be stiff. Under these conditions, the movement of molecules as a single unit is still successfully described by the equations of motion, although this does not apply to individual atoms, because their natural vibrations are constrained to be zero. Such constraints are introduced in the equations of motion in the form of extra energy terms. The major drawback of this approach is that extracting further information from the trajectory, like temperature, pressure, or energy, also requires to account for the constraints. Another approach is to coarse grain the potentials, ${ }^{60}$ which removes from the simulation those atoms that have no effect, or more precisely, combines them with their parent atoms as a rigid body. For instance, neutral $\mathrm{H}$ atoms can be considered as part of the carbon atom they are attached to. By doing this, the system is greatly simplified without the need to modify the equations of motion. The only condition is to include 
properly parametrised rigid bodies in the force field.

As introduced at the beginning of this section, atomic interactions in MD are treated differently depending on the distance between the atoms. In theory, an atom exerts a force on any other atom of the system. If said atoms are far apart, the force and the energy associated with their interaction might be small, but given the large number of long-range interactions that are present in a system, this term is not negligible and must be computed in order to derive proper energy values. This implies a large number of calculations and several strategies have been developed to decrease the computational cost. ${ }^{59}$ Lennard-Jones interactions (Eq. 1.1) are simply ignored over a certain distance, under the assumption that the neglected contributions are likely to cancel out when added together. The energy of these interactions varies with $r^{-6}$, hence it is easy to stablish a cutoff distance over which the energy is statistically negligible. Coulombic interactions (Eq. 1.2) on the other hand, vary with $r^{-1}$, which implies a much slower decrease of energy with distance, making it more complex to establish a clear cutoff. These interactions are computed by means of Ewald summation. Ewald summation divides the electrostatic interactions in two terms: short- and long-range. The first term is computed using the Coulomb equation (Eq. 1.2) for a small number of atom pairs which are closer than a certain cutoff. The number of atom pairs over the cutoff is much larger and in the Ewald scheme their interactions are computed in reciprocal space, as convergence is much faster, making use of the fact that the system is considered periodic during the simulation. The implementation of the Ewald summation most frequently found in MD motors is particle mesh Ewald (PME). The main characteristic of this technique is that the computation is speeded up by using fast Fourier transform, which does not compute each interaction independently, but rather distributes them over the nodes of an imaginary grid, performing the calculation only at the nodes of the grid. Ewald summation is the most time-consuming part of any MD simulation.

The use of Ewald summation requires the simulated system to be in periodic boundary conditions (PBC). These conditions assume that the content of the simulation box is repeated infinitely in all three directions 
of space, and that when a particle leaves the box from one side, it simple returns from the opposite side, maintaining its energy and trajectory. $\mathrm{PBC}$ not only reduce the computational cost of the simulation, but are also convenient to avoid undesired effects at the surface of the simulation box. Nevertheless, the use of PBC can also cause errors. For instance, in a simulation box containing a molecule or a cluster of molecules surrounded by solvent, the box must be large enough to avoid the molecules interacting with their images in the neighbouring box, which is physically unsound. In the absence of solvent, the simulation box must be large enough to accurately represent disorder.

\subsubsection{Trajectory analysis}

MD simulations of molecular systems produce trajectories in terms of position and velocity of atoms. For the simulation of crystals, as is the case in this work, it is usually more interesting to look at where the atoms move rather than the velocity with which they move. A common first step is a visual analysis of the trajectories, which can be watched as a video. When events of interest are found, the geometrical information can be extracted and processed to quantify the event. Because of the very specific nature of each event studied in this work, custom analysis scripts have been written tailored to each case. A detailed description of each analysis script is given in the Experimental section of each appropriate chapter.

\subsubsection{Energy calculations}

According to statistical mechanics, the energy of an $N V E$ ensemble is:

$$
\begin{gathered}
\mathrm{d} E=T \mathrm{~d} S-P \mathrm{~d} V+\mu \mathrm{d} N \quad \Rightarrow \\
(\mathrm{d} E)_{N V}=T(\mathrm{~d} S)_{N V}
\end{gathered}
$$

From here, the temperature is:

$$
T=\left(\frac{\partial E}{\partial S}\right)_{N V}
$$


Entropy is defined as:

$$
S=k_{\mathrm{B}} \ln \Omega
$$

Where $\Omega$ is the $N V E$ partition function. Combining with Eq. 1.13:

$$
\frac{1}{T}=\left(\frac{\partial S}{\partial E}\right)_{N V}=\left(\frac{\partial}{\partial E}\left(k_{\mathrm{B}} \ln \Omega\right)\right)_{N V}=k_{\mathrm{B}}\left(\frac{\partial(\ln \Omega)}{\partial E}\right)_{N V}
$$

From which the thermodynamic $\beta$ can be defined:

$$
\beta=\frac{1}{T k_{\mathrm{B}}}=\left(\frac{\partial(\ln \Omega)}{\partial E}\right)_{N V}
$$

Given an $N V T$ system consisting of a small simulation box, which can be in $\nu$ states of energy $E_{\nu}$, immersed in a bath large enough so that its temperature can be considered constant in comparison to that of the box, the entropy of the bath is:

$$
S_{\text {bath }}=k_{\mathrm{B}} \ln \Omega_{\text {bath }}
$$

And its energy is:

$$
\begin{gathered}
E_{\text {system }}=E_{\text {bath }}+E_{\text {box }}=E_{\text {bath }}+E_{\nu} \quad \Rightarrow \\
E_{\text {bath }}=E_{\text {system }}-E_{\nu}
\end{gathered}
$$

The value of the entropy at $E_{\text {bath }}$ can be approximated by two terms of a Taylor expansion:

$$
\begin{aligned}
S_{\text {bath }}\left(E_{\text {bath }}\right)= & S_{\text {bath }}\left(E_{\text {system }}-E_{\nu}\right) \approx \\
& S_{\text {bath }}\left(E_{\text {system }}\right)-E_{\nu} \frac{\partial S_{\text {bath }}}{\partial E_{\text {system }}}
\end{aligned}
$$

From Eqs. 1.13 and 1.16:

$$
\begin{aligned}
S_{\text {bath }}\left(E_{\text {bath }}\right) \approx & S_{\text {bath }}\left(E_{\text {system }}\right)-\frac{E_{\nu}}{T}= \\
& S_{\text {bath }}\left(E_{\text {system }}\right)-\beta k_{\mathrm{B}} E_{\nu}
\end{aligned}
$$


Applying to Eq. 1.17:

$$
\begin{gathered}
\ln \Omega_{\text {bath }}=\frac{1}{k_{\mathrm{B}}} S_{\text {bath }} \approx \frac{1}{k_{\mathrm{B}}} S_{\text {bath }}\left(E_{\text {system }}\right)-\beta E_{\nu} \Rightarrow \\
\Omega_{\text {bath }} \approx \exp \left(\frac{1}{k_{\mathrm{B}}} S_{\text {bath }}\left(E_{\text {system }}\right)-\beta E_{\nu}\right) \propto \mathrm{e}^{-\beta E_{\nu}}
\end{gathered}
$$

Where $e^{-\beta E_{\nu}}$ is the so called Boltzmann factor. The total number of states of the system is given by:

$$
\begin{gathered}
\Omega_{\text {system }}=\Omega_{\text {bath }} \Omega_{\text {box }} \quad \Rightarrow \\
\Omega_{\text {box }}=\frac{\Omega_{\text {system }}}{\Omega_{\text {bath }}}
\end{gathered}
$$

The probability of the simulation box being in a given state $\nu$, applying Eq. 1.21, is:

$$
P_{\nu}=\frac{1}{\Omega_{\text {box }}}=\frac{\Omega_{\text {bath }}}{\Omega_{\text {system }}}=\frac{\mathrm{e}^{-\beta E_{\nu}}}{\sum_{\nu} \mathrm{e}^{-\beta E_{\nu}}}=\frac{1}{Q} \mathrm{e}^{-\beta E_{\nu}}
$$

Where $Q$, the partition function of an $N V T$ ensemble, is:

$$
Q=\Omega_{\text {system }}=\sum_{\nu} \mathrm{e}^{-\beta E_{\nu}}
$$

A partition function allows to compute average properties of a system. For an $N V T$ ensemble:

$$
\langle X\rangle=\frac{1}{Q} \sum_{\nu} X_{\nu} \mathrm{e}^{-\beta E_{\nu}}
$$

Then, the average energy of the system is:

$$
\langle E\rangle=\frac{1}{Q} \sum_{\nu} E_{\nu} \mathrm{e}^{-\beta E_{\nu}}
$$

The derivative of the Boltzmann factor with respect to $\beta$ is:

$$
\left(\frac{\partial\left(\mathrm{e}^{-\beta E_{\nu}}\right)}{\partial \beta}\right)_{N V}=-E_{\nu} \mathrm{e}^{-\beta E_{\nu}}
$$


Applying to Eq. 1.26:

$$
\begin{aligned}
\langle E\rangle= & \frac{1}{Q} \sum_{\nu}-\left(\frac{\partial\left(\mathrm{e}^{-\beta E_{\nu}}\right)}{\partial \beta}\right)_{N V}= \\
& -\frac{1}{Q}\left(\frac{\partial}{\partial \beta} \sum_{\nu} \mathrm{e}^{-\beta E_{\nu}}\right)_{N V}
\end{aligned}
$$

Applying Eq. 1.24:

$$
\langle E\rangle=-\frac{1}{Q}\left(\frac{\partial Q}{\partial \beta}\right)_{N V}=-\left(\frac{\partial(\ln Q)}{\partial \beta}\right)_{N V}
$$

It can be demonstrated that $Q$ is related to the Helmholtz free energy:

$$
\begin{gathered}
A=E-T S \Rightarrow \\
\beta A=\beta E-\beta T S=\beta E-\frac{1}{k_{\mathrm{B}}} S
\end{gathered}
$$

Taking derivatives with respect to $\beta$ :

$$
\begin{aligned}
\left(\frac{\partial(\beta A)}{\partial \beta}\right)_{N V}= & \left(\frac{\partial(\beta E)}{\partial \beta}\right)_{N V}-\left[\frac{\partial}{\partial \beta}\left(\frac{1}{k_{\mathrm{B}}} S\right)\right]_{N V}= \\
& E+\beta\left(\frac{\partial E}{\partial \beta}\right)_{N V}-\frac{1}{k_{\mathrm{B}}}\left(\frac{\partial S}{\partial \beta}\right)_{N V}
\end{aligned}
$$

From Eq. 1.16, the differential of $\beta$ can be expressed in terms of direct temperature:

$$
\begin{gathered}
\beta=\frac{1}{T k_{\mathrm{B}}} \Rightarrow \\
\frac{\partial \beta}{\partial T}=\frac{\partial}{\partial T}\left(\frac{1}{T k_{\mathrm{B}}}\right)=-\frac{1}{T^{2} k_{\mathrm{B}}} \Rightarrow \\
\partial \beta=\frac{-\partial T}{T^{2} k_{\mathrm{B}}}
\end{gathered}
$$

Applying to Eq. 1.31:

$$
\begin{aligned}
\left(\frac{\partial(\beta A)}{\partial \beta}\right)_{N V}= & E+\beta\left(\frac{\partial E}{-(\partial T) / T^{2} k_{\mathrm{B}}}\right)_{N V}- \\
& \frac{1}{k_{\mathrm{B}}}\left(\frac{\partial S}{-(\partial T) / T^{2} k_{\mathrm{B}}}\right)_{N V}= \\
& E-T\left(\frac{\partial E}{\partial T}\right)_{N V}+T^{2}\left(\frac{\partial S}{\partial T}\right)_{N V}
\end{aligned}
$$


By definition:

$$
C_{V}=\left(\frac{\partial E}{\partial T}\right)_{V}
$$

And then, recalling Eq. 1.12:

$$
\begin{gathered}
(\mathrm{d} E)_{N V}=T(\mathrm{~d} S)_{N V} \Rightarrow \\
\left(\frac{\partial E}{\partial T}\right)_{N V}=T\left(\frac{\partial S}{\partial T}\right)_{N V} \Rightarrow \\
\left(\frac{\partial S}{\partial T}\right)_{N V}=\frac{1}{T}\left(\frac{\partial E}{\partial T}\right)_{N V}=\frac{C_{V}}{T}
\end{gathered}
$$

Applying Eqs. 1.34 and 1.35 to Eq. 1.33 and equating the macroscopic ensemble to the microscopic level:

$$
\left(\frac{\partial(\beta A)}{\partial \beta}\right)_{N V}=E-T C_{V}+T C_{V}=E=\langle E\rangle
$$

Bringing together Eqs. 1.29 and 1.36:

$$
\begin{gathered}
\langle E\rangle=-\left(\frac{\partial(\ln Q)}{\partial \beta}\right)_{N V}=\left(\frac{\partial(\beta A)}{\partial \beta}\right)_{N V} \Rightarrow \\
\ln Q=-\beta A \Rightarrow \\
Q=\mathrm{e}^{-\beta A}
\end{gathered}
$$

In a continuous system, $Q$ can be integrated over all positions of all particles of the system:

$$
Q=C \int \mathrm{e}^{-\beta E_{\nu}} \mathrm{d} x_{1} \mathrm{~d} y_{1} \mathrm{~d} z_{1} \ldots \mathrm{d} x_{N} \mathrm{~d} y_{N} \mathrm{~d} z_{N}
$$

Where $C$ is a constant including the kinetic energy term and the number of particles. Defining the reduced position $s$ as:

$$
\begin{gathered}
s_{n}=\frac{x_{n}}{L_{x}} \frac{y_{n}}{L_{y}} \frac{z_{n}}{L_{z}} \Rightarrow \\
x_{n} y_{n} z_{n}=L_{x} L_{y} L_{z} s_{n}=V s_{n} \quad \Rightarrow \\
\mathrm{d} x_{n} \mathrm{~d} y_{n} \mathrm{~d} z_{n}=V \mathrm{~d} s_{n}
\end{gathered}
$$

Where $L_{x}, L_{y}$, and $L_{z}$ are the dimensions of the system. Applying to 
Eq. 1.38:

$$
Q=C \int \mathrm{e}^{-\beta E_{\nu}} V^{N} \mathrm{~d}^{3 N} s=C V^{N} \int \mathrm{e}^{-\beta U_{\nu}} \mathrm{d}^{3 N} s
$$

Given an NPT ensemble, consisting of a small box of variable volume, containing $N$ particles, immersed in a large bath also of variable volume, containing $M$ ideal particles, large enough so that its temperature can be considered constant in comparison to that of the box:

$$
\begin{gathered}
V_{\text {system }}=V_{\text {bath }}+V_{\text {box }} \Rightarrow \\
V_{\text {bath }}=V_{\text {system }}-V_{\text {box }}=V_{0}-V
\end{gathered}
$$

In terms of partition functions, applying Eq. 1.40:

$$
\begin{aligned}
Q_{\text {system }}= & Q_{\text {bath }} Q_{\text {box }}= \\
& C V_{\text {bath }}^{M} \int \mathrm{e}^{-\beta U_{\nu}} \mathrm{d}^{3 M} s \quad C V_{\text {box }}^{N} \int \mathrm{e}^{-\beta U_{\nu}} \mathrm{d}^{3 N} s= \\
& C\left(V_{0}-V\right)^{M} \int \mathrm{e}^{-\beta U_{\nu}} \mathrm{d}^{3 M^{M}} s \quad C V^{N} \int \mathrm{e}^{-\beta U_{\nu}} \mathrm{d}^{3 N} s
\end{aligned}
$$

Because the particles in the bath are ideal, their potential energy is zero:

$$
Q_{\text {system }}=C\left(V_{0}-V\right)^{M} V^{N} \int \mathrm{e}^{-\beta U_{\nu}} \mathrm{d}^{3 N} s
$$

Assuming that $V_{0} \gg V$ and $M \rightarrow \infty$ :

$$
\begin{aligned}
\left(V_{0}-V\right)^{M}= & V_{0}^{M}\left(\frac{V_{0}}{V_{0}}-\frac{V}{V_{0}}\right)^{M}=V_{0}^{M}\left(1-\frac{V}{V_{0}}\right)^{M} \\
& \approx V_{0}^{M} \exp \left(\frac{-M V}{V_{0}}\right)
\end{aligned}
$$

From the law of ideal gases:

$$
\begin{aligned}
P V_{0} & =M k_{\mathrm{B}} T \Rightarrow \\
\frac{M}{V_{0}} & =\frac{P}{T k_{\mathrm{B}}}=\beta P
\end{aligned}
$$

Applying to Eq. 1.44:

$$
\left(V_{0}-V\right)^{M} \approx V_{0}^{M} \mathrm{e}^{-\beta P V}
$$


And applying to Eq. 1.43:

$$
\begin{aligned}
Q_{\text {system }}= & C V_{0}^{M} \mathrm{e}^{-\beta P V} V^{N} \int \mathrm{e}^{-\beta U_{\nu}} \mathrm{d}^{3 N} s= \\
& C V_{0}^{M} V^{N} \int \mathrm{e}^{-\beta U_{\nu}} \mathrm{e}^{-\beta P V} \mathrm{~d}^{3 N} s= \\
& C V_{0}^{M} V^{N} \int \mathrm{e}^{-\beta U_{\nu}-\beta P V} \mathrm{~d}^{3 N} s
\end{aligned}
$$

By analogy with $Q$, for an NPT ensemble, the partition function $Z$ is defined as:

$$
Z=\sum_{\nu} \mathrm{e}^{-\beta U_{\nu}-\beta P V}
$$

The average volume of an NPT ensemble is:

$$
\langle V\rangle=\frac{1}{Z} \sum_{\nu} V \mathrm{e}^{-\beta U_{\nu}-\beta P V}
$$

The derivative of the Boltzmann factor with respect to $\beta P$ is:

$$
\left(\frac{\partial\left(\mathrm{e}^{-\beta U_{\nu}-\beta P V}\right)}{\partial(\beta P)}\right)_{N T}=-V \mathrm{e}^{-\beta U_{\nu}-\beta P V}
$$

Applying to Eq. 1.49:

$$
\begin{aligned}
\langle V\rangle= & -\frac{1}{Z} \sum_{\nu}\left(-\frac{\partial\left(\mathrm{e}^{-\beta U_{\nu}-\beta P V}\right)}{\partial(\beta P)}\right)_{N T}= \\
& -\frac{1}{Z}\left(\frac{\partial}{\partial(\beta P)} \sum_{\nu} \mathrm{e}^{-\beta U_{\nu}-\beta P V}\right)_{N T}
\end{aligned}
$$

Applying Eq. 1.48:

$$
\langle V\rangle=-\frac{1}{Z}\left(\frac{\partial Z}{\partial(\beta P)}\right)_{N T}=-\left(\frac{\partial(\ln Z)}{\partial(\beta P)}\right)_{N T}
$$

It can be demonstrated that $Z$ is related to the Gibbs free energy:

$$
\begin{gathered}
G=H-T S=E+P V-T S \Rightarrow \\
\beta G=\beta E+\beta P V-\beta T S=\beta E+\beta P V-\frac{1}{k_{\mathrm{B}}} S
\end{gathered}
$$

Taking derivatives with respect to $\beta P$ and equating the macroscopic 
ensemble to the microscopic level:

$$
\begin{aligned}
\left(\frac{\partial(\beta G)}{\partial(\beta P)}\right)_{N T}= & \left(\frac{\partial(\beta E)}{\partial(\beta P)}\right)_{N T}+\left(\frac{\partial(\beta P V)}{\partial(\beta P)}\right)_{N T}^{-} \\
& {\left[\frac{\partial}{\partial(\beta P)}\left(\frac{1}{k_{\mathrm{B}}} S\right)\right]_{N T}=V=\langle V\rangle }
\end{aligned}
$$

Bringing together Eqs. 1.52 and 1.54:

$$
\begin{gathered}
\langle V\rangle=-\left(\frac{\partial(\ln Z)}{\partial(\beta P)}\right)_{N T}=\left(\frac{\partial(\beta G)}{\partial(\beta P)}\right)_{N T} \Rightarrow \\
\ln Z=-\beta G \Rightarrow \\
Z=\mathrm{e}^{-\beta G}
\end{gathered}
$$

Differently from geometrical calculations, energy calculations are standardised to a great extent. The simplest approach is applied to frequent events. The idea is to perform a simulation, assuming that the event of interest will take place a reasonable number of times during the entire length of the simulation. From Eq. 1.55, an NPT system, as used in this work, evolving along a certain trajectory $\lambda$, is described by:

$$
Z(\lambda)=\mathrm{e}^{-\beta G(\lambda)}
$$

If the trajectory $\lambda$ is not known, it can be described in terms of an order parameters $\chi$, whose value can be computed from the trajectory a posteriori:

$$
Z(\chi)=\mathrm{e}^{-\beta G(\chi)}
$$

The total partition function is the sum over all possible values of $\chi$ :

$$
Z=\sum_{\chi} Z(\chi)=\sum_{\chi} \mathrm{e}^{-\beta G(\chi)}
$$

Once the partition functions are defined, it is possible to calculate the probability of any state $\chi_{i}$ as the ratio between its partition function and the total partition function:

$$
P\left(\chi_{i}\right)=\frac{Z\left(\chi_{i}\right)}{Z}=\frac{\mathrm{e}^{-\beta G\left(\chi_{i}\right)}}{\sum_{\chi} \mathrm{e}^{-\beta G(\chi)}}
$$


And the relative probability of two states $\chi_{i}$ and $\chi_{j}$ is:

$$
\begin{aligned}
& \frac{P\left(\chi_{i}\right)}{P\left(\chi_{j}\right)}= \frac{\sum_{\chi} \mathrm{e}^{-\beta G(\chi)}}{\frac{\mathrm{e}^{-\beta G\left(\chi_{j}\right)}}{\sum_{\chi} \mathrm{e}^{-\beta G(\chi)}}}=\frac{\mathrm{e}^{-\beta G\left(\chi_{i}\right)}}{\mathrm{e}^{-\beta G\left(\chi_{j}\right)}}= \\
& \mathrm{e}^{-\beta\left[G\left(\chi_{i}\right)-G\left(\chi_{j}\right)\right]}=\mathrm{e}^{-\beta \Delta G\left(\chi_{i j}\right)}
\end{aligned}
$$

And finally:

$$
\Delta G\left(\chi_{i j}\right)=-\frac{1}{\beta} \ln \frac{P\left(\chi_{i}\right)}{P\left(\chi_{j}\right)}
$$

Taking the most likely state as the reference value to determine Gibbs free energy differences: ${ }^{61}$

$$
\Delta G\left(\chi_{i}\right)=-\frac{1}{\beta} \ln \frac{P\left(\chi_{i}\right)}{P\left(\chi_{\max }\right)}
$$

This equation allows to extract energy values directly from the likelihood of each state, which can be measured from the simulation. For a given event of interest, it is enough to determine how likely is it in the simulation, and then convert the probability to an energy term. In practice, the best approach is to obtain the probabilities of all related events (for instance, molecular reorientation, torsion angle rotation, formation of $\mathrm{H}$ bonds, etc.) and create a histogram, from which an energy curve can be directly computed.

The scheme for energy calculations outlined above requires a large sampling of all possible states, which in turn requires the events of interest to be as likely as any other event. Unfortunately, the events of interest can be very unlikely, so called rare events, and might not take place a significant number of times during the short time scale which is feasible in an MD simulation. For these situations, it is possible to use steered MD simulations. The idea behind a steered simulation is to input certain energy constraints to the system to force rare events to occur. Knowing the energy of the system during the trajectory and the extra energy input as a constraint, it is possible to determine the energy of the event. 
Two very common schemes for steered MD are umbrella sampling and metadynamics.

Umbrella sampling ${ }^{59,62}$ works under the assumption that the trajectory of the event is known, at least approximately. The event is defined in terms of an order parameter, and potential wells are applied to keep this order parameter evolving along the trajectory, effectively forcing the system to reproduce the event. By discounting the energy of the constraints from the total energy of the trajectory it is possible to know the energy landscape of the event. In practice, this is done by weighted histogram analysis method (WHAM). ${ }^{63}$ Umbrella sampling provides a fast way to measure energy curves, but its major drawback is that it is necessary to know in advance the trajectory of the event.

For those events whose trajectory is unknown, metadynamics ${ }^{64}$ offers a good alternative. In this scheme, an order parameter related to the event of interest is chosen an forced to map the entire energy landscape. For this, Gaussian hills are applied to the order parameter, forcing it to change and explore all possible states. Analogously to umbrella sampling, knowing the amount of energy input as Gaussian hills and the energy of the simulated trajectory, it is possible to retrieve the energy associated only to the order parameter. The main disadvantage of metadynamics is that it requires long simulation times.

\subsubsection{Addressing the validity of molecular dynamics simulations}

MD simulations are based on theoretical models, and as such, the validity of predicted trajectories should be compared with experimental results when feasible. Due to the very different nature of computational and experimental observations, comparison of both is far from being trivial, ${ }^{65,66}$ especially considering that computational techniques are often used to access information which is not available experimentally. Experience has shown that upon a careful parametrisation, MD simulations can accurately reproduce crystal structures to the level of anisotropic displacement parameters. ${ }^{67,68}$ Nevertheless, unless all relevant parameters are fine tuned, simulated results are better used in a qualitative man- 
ner, ${ }^{67}$ as most force fields had proven to reproduce the overall behaviour of molecular systems reasonably well. ${ }^{69-71}$ Free energy calculations are particularly sensitive to the choice of force field and parameters, and results are difficult to validate experimentally. ${ }^{72}$ In this work, Gibbs free energy differences determined from MD simulations are reported, mainly as a guidance, and discussion of results relies principally on overall shape of the free energy surfaces (FESs).

A common quick validation procedure, after ensuring that bond lengths and angles are properly reproduced, consists in checking how simulated unit-cell parameters compare with experimental ones. Unfortunately, most force fields oriented towards simulation of organic molecules have not been consistently tested at the pressures used in this work - up to 1.2 GPa. Instabilities arising from the use of extreme external conditions renders the comparison of unit-cell dimensions unreliable, a topic commonly addressed in the discussion forums of the different MD motors. For instance, at high pressure local densities might increase more than expected from experimental observations, creating vacuum regions in the model. Subsequently, the simulation box will shrink suddenly to eliminate such regions. This problem has been dealt with in the present work by minimising the energy of all molecular models under simulation conditions prior to the production runs, not to reach a real energy minimum, but to overcome the aforementioned instabilities. ${ }^{67}$ As a result, at the start of the production runs unit-cell parameters of the model might be significantly different from the ones measured by X-ray diffraction. Nevertheless, molecular conformations and intermolecular distances at this stage are reproduced reasonably well. 


\section{References}

[1] C. E. Weir, E. R. Lippincott, A. van Valkenburg and E. N. Bunting, J. Res. Nat. Bur. Stand., 1959, 63A, 55-62.

[2] P. W. Bridgman and I. Simon, J. Appl. Phys., 1953, 24, 405-413.

[3] G. J. Piermarini, J. Res. Natl. Inst. Stand. Technol., 2001, 106, 889-920.

[4] L. Merrill and W. A. Bassett, Rev. Sci. Instrum., 1974, 45, 290-294.

[5] R. M. Hazen and L. W. Finger, Carnegie Inst. Washington Yearb., 1977, 76, 655-656.

[6] W. A. Bassett, High Pressure Res., 2009, 29, 163-186.

[7] R. Boehler and K. de Hantsetters, High Pressure Res., 2004, 24, 391-396.

[8] A. Katrusiak, Acta Cryst., 2008, A64, 135-148.

[9] H. Ahsbahs, Z. Kristallogr., 2004, 219, 305-308.

[10] D. R. Allan, R. Miletich and R. J. Angel, Rev. Sci. Instrum., 1996, 67, 840-842.

[11] R. Boehler, Rev. Sci. Instrum., 2006, 77, 2006-2008.

[12] T. Kenichi and N. Satoshi, Rev. Sci. Instrum., 2003, 74, 3017-3020.

[13] D. J. Dunstan, Rev. Sci. Instrum., 1989, 60, 3789-3795.

[14] G. J. Piermarini, S. Block, J. D. Barnett and R. A. Forman, J. Appl. Phys., 1975, 46, 2774-2780.

[15] H. K. Mao, J. Xu and P. M. Bell, J. Geophys. Res., 1986, 91, 46734676.

[16] R. Miletich, D. R. Allan and W. F. Kuhs, Reviews Miner. Geochem., 2000, 41, 445-519.

[17] P. M. Oger, I. Daniel and A. Picard, BBA Proteins Proteom., 2006, 1764, 434-442.

[18] D. E. Graf, R. L. Stillwell, K. M. Purcell and S. W. Tozer, High Pressure Res., 2011, 31, 533-543.

[19] C. A. Cameron, PhD thesis, University of Edinburgh, 2014.

[20] J. M. Besson, R. J. Nelmes, G. Hamel, J. S. Loveday, G. Weill and S. Hull, Physica B, 1992, 180-181, 907-910. 
[21] J. Binns, K. V. Kamenev, G. J. McIntyre, S. A. Moggach and S. Parsons, IUCrJ, 2016, 3, 1-12.

[22] E. V. Boldyreva, Acta Cryst., 2008, A64, 218-231.

[23] M. A. Neumann, J. van de Streek, F. P. A. Fabbiani, P. Hidber and O. Grassmann, Nat. Commun., 2015, 6, 7793.

[24] S. Saouane and F. P. A. Fabbiani, Cryst. Growth Des., 2015, 15, $3875-3884$.

[25] F. P. A. Fabbiani, G. Buth, D. C. Levendis and A. J. Cruz-Cabeza, ChemComm, 2014, 50, 1817-1819.

[26] F. P. A. Fabbiani, G. Buth, B. Dittrich and H. Sowa, CrystEngComm, 2010, 12, 2541-2550.

[27] F. P. A. Fabbiani, D. C. Levendis, G. Buth, W. F. Kuhs, N. Shanklandd and H. Sowa, CrystEngComm, 2010, 12, 2354-2360.

[28] F. P. A. Fabbiani and C. R. Pulham, Chem. Soc. Rev., 2006, 35, 932-942.

[29] H. Tomkowiak, A. Olejniczak and A. Katrusiak, Cryst. Growth Des., 2013, 13, 121-125.

[30] M. Andrzejewski, A. Olejniczak and A. Katrusiak, Cryst. Growth Des., 2011, 11, 4892-4899.

[31] A. Olejniczak and A. Katrusiak, Cryst. Growth Des., 2011, 11, 2250-2256.

[32] B. F. Johnston, W. G. Marshall, S. Parsons, A. J. Urquhart and I. D. H. Oswald, J. Phys. Chem. B, 2014, 118, 4044-4051.

[33] A. J. Graham, D. R. Allan, A. Muszkiewicz, C. A. Morrison and S. A. Moggach, Angew. Chem. Int. Ed., 2011, 50, 11138-11141.

[34] F. P. A. Fabbiani, D. R. Allan, W. I. F. David, A. J. Davidson, A. R. Lennie, S. Parsons, C. R. Pulham, J. E. Warren, R. David, I. F. William, J. Alistair, A. R. Lennie, S. Parsons, C. R. Pulham and J. E. Warren, Cryst. Growth Des., 2007, 7, 1115-1124.

[35] S. A. Moggach, D. R. Allan, S. J. Clark, M. J. Gutmann, S. Parsons, C. R. Pulham and L. Sawyer, Acta Cryst., 2006, B62, 296-309.

[36] S. A. Moggach, W. G. Marshall and S. Parsons, Acta Cryst., 2006, 62, 815-825. 
[37] F. P. A. Fabbiani, D. R. Allan, S. Parsons and C. R. Pulham, Acta Cryst., 2006, B62, 826-842.

[38] V. S. Minkov, S. V. Goryainov, E. V. Boldyreva and C. H. Görbitz, J. Raman Spectrosc., 2010, 41, 1748-1758.

[39] E. V. Boldyreva, J. Mol. Struct., 2003, 647, 159-179.

[40] E. V. Boldyreva, T. P. Shakhtshneider, H. Ahsbahs, H. Sowa and H. Uchtmann, J. Therm. Anal. Calorim., 2002, 68, 437-452.

[41] D. Seoung, Y. Lee, C.-C. Kao and T. Vogt, Chem. Eur. J., 2013, 19, 10876-10883.

[42] Y. Lee, T. Vogt, J. A. Hriljac, J. B. Parise, J. C. Hanson and S. J. Kim, Nature, 2002, 420, 485-489.

[43] R. J. Angel, N. L. Ross, I. G. Wood and P. A. Woods, Phase Transit., 1992, 39, 13-32.

[44] A. Katrusiak, Acta Cryst., 2004, A60, 409-417.

[45] R. J. Angel, J. Appl. Cryst., 2004, 37, 486-492.

[46] A. Dawson, D. R. Allan, S. Parsons and M. Ruf, J. Appl. Cryst., 2004, 37, 410-416.

[47] P. Dera and A. Katrusiak, J. Appl. Cryst., 1999, 32, 510-515.

[48] Bruker-AXS, SAINT+ Integrating Engine v8.18C, Bruker-AXS, Madison, Wisconsin, USA, 2011.

[49] CRYSALIS PRO v171.36.28, Rigaku Oxford Diffraction, Tokyo, Japan, 2012.

[50] S. Parsons, SHADE, The University of Edinburgh, Edinburgh, United Kingdom, 2005.

[51] G. M. Sheldrick, SADABS v2008-1, Bruker-AXS, Madison, Wisconsin, USA, 2008.

[52] G. M. Sheldrick, TWINABS v2008-1, Bruker-AXS, Madison, Wisconsin, USA, 2008.

[53] R. H. Blessing, Cryst. Rev., 1987, 1, 3-58.

[54] G. M. Sheldrick, XPREP v2012-1, Bruker-AXS, Madison, Wisconsin, USA, 2012.

[55] G. M. Sheldrick, Acta Cryst., 2015, C71, 3-8.

[56] B. J. Alder and T. E. Wainwright, J. Chem. Phys., 1959, 31, 459466. 
[57] J. C. Phillips, R. Braun, W. Wang, J. Gumbart, E. Tajkhorshid, E. Villa, C. Chipot, R. D. Skeel, L. Kalé and K. Schulten, J. Comput. Chem., 2005, 26, 1781-1802.

[58] M. J. Abraham, T. Murtola, R. Schulz, S. Páll, J. C. Smith, B. Hess and E. Lindah, SoftwareX, 2015, 1-2, 19-25.

[59] D. Frenkel and B. Smit, Understanding Molecular Simulation: From Algorithms to Applications, Academic Press, 2001.

[60] M. P. Allen, Computational Soft Matter: From Synthetic Polymers to Proteins, Lecture Notes, John von Neumann Institute for Computing, Jülich, 2004, pp. 1-28.

[61] J. G. Kirkwood, J. Chem. Phys., 1935, 3, 300-313.

[62] G. M. Torrie and J. P. Valleau, J. Comput. Phys., 1977, 23, 187199.

[63] S. Kumar, J. M. Rosenberg, D. Bouzida, R. H. Swendsen and P. A. Kollman, J. Comput. Chem., 1992, 13, 1011-1021.

[64] A. Laio and M. Parrinello, Proc. Nat. Acad. Sci. USA, 2002, 99, $12562-12566$.

[65] W. F. van Gunsteren and A. E. Mark, J. Chem. Phys., 1998, 108, 6109-6116.

[66] W. F. van Gunsteren, J. Dolenc and A. E. Mark, Curr. Opin. Struct. Biol., 2008, 18, 149-153.

[67] A. Nemkevich, H. B. Bürgi, M. A. Spackman and B. Corry, Phys. Chem. Chem. Phys, 2010, 12, 14916-14929.

[68] G. M. Lombardo, G. Portalone, M. Colapietro, A. Rescifina and F. Punzo, J. Mol. Struct., 2011, 994, 87-96.

[69] O. Guvench, E. Hatcher, R. M. Venable, R. W. Pastor and A. D. MacKerell, J. Chem. Theory Comput., 2009, 5, 2353-2370.

[70] N. Schmid, A. P. Eichenberger, A. Choutko, S. Riniker, M. Winger, A. E. Mark and W. F. Van Gunsteren, Eur. Biophys. J., 2011, 40, 843-856.

[71] W. L. Jorgensen, D. S. Maxwell and J. Tirado-Rives, J. Am. Chem. Soc., 1996, 118, 11225-11236.

[72] N. Hansen and W. F. Van Gunsteren, J. Chem. Theory Comput., 2014, 10, 2632-2647. 


\section{Formation of hydrates at high}

pressure

\subsection{Introduction}

CSP is the prediction of the crystal structure of a material based on the chemical diagram of its constituents. ${ }^{1}$ Several computer programs have been developed for this task. ${ }^{2}$ CSP is the subject of extensive research nowadays, as it provides another approach, complimentary to experimental research, to problems which are of great financial interest for certain industries. For instance, polymorph selection, a common problem in the pharmaceutical industry, ${ }^{3-5}$ can be optimised if both in silico and experimental polymorph screening results are available. Similarly, cocrystal and solvate screening will also benefit from accurate CSP. It can also be used to calculate physicochemical properties of materials without the need of crystallisation, with the consequential economic saving. Despite all its advantages, CSP has proven so challenging as to be described as hopelessly difficult. ${ }^{6}$ 
In the field of molecular materials, CSP has proven successful on predicting the structure of crystals containing molecules which are either small or exhibit limited flexibility. ${ }^{2}$ Recent results show that certain approaches are also able to tackle the prediction of larger molecules ${ }^{7}$ and multicomponent systems, such as solvates and cocrystals. ${ }^{8}$ The rate of success on predicting the structure of hydrates is acceptable, ${ }^{2,9-12}$ although the problem remains challenging, because the number of candidate structures that need to be evaluated grows exponentially with the number of chemical species in the crystal.

Data mining strategies have been suggested as a way to reduce the number of candidates and optimise the search. ${ }^{2}$ Data mining is based on the definition of structural predictors, which are evaluated over large datasets - the Cambridge Structural Database (CSD) for instance - by means of statistics, artificial intelligence and machine learning techniques. The predictors are then used to limit the search space to the most probable structures. Descriptors can be based on statistical distributions of structures ${ }^{13}$ or physical properties, like intermolecular distances and geometries,${ }^{10,14}$ packing densities, ${ }^{10}$ or space groups. ${ }^{15}$ Actual accuracy of CSP based on data mining for solvate formation is around $75 \% .{ }^{13}$ CSP will benefit from better structural descriptors, which in turn rely on the continuous expansion and study of the CSD.

The biggest challenge of CSP is that it is becoming increasingly successful at computing accurate crystal energy landscapes, without being able to asses whether the lowest energy form will crystallise at all. Phase stability can be assessed by lattice and free energy calculations, ${ }^{16}$ which are mainly limited by computational power, while modelling crystallisation is a more complex process which largely relies on kinetics, for which few models are available. ${ }^{17}$ With regard to hydrates of organics molecules, substantial exploration of the CSD has been performed, in order to better understand how they form, and expecting that the gained knowledge will help to predict crystal structures along with the likelihood of their crystallisation.

The first surveys of the CSD showed that water tends to be a stronger H-bond acceptor than donor, ${ }^{18}$ that H-bonded networks tend to use 
all available donors and acceptors, ${ }^{19}$ and that formation of $\mathrm{H}$ bonds is related to the ratio of donors and acceptors in a given molecule and the weight of the molecule. ${ }^{20}$ More recently, a systematic analysis of the geometry of H-bonded water molecules in organic crystals found a mild correlation between their geometry and their chemical environment. ${ }^{21,22}$ Although such an observation is not useful for prediction of hydrate formation, it led to a deeper analysis of chemical environments in hydrate structures, revealing that certain functional groups promoted hydration. ${ }^{23,24}$ The last work of Infantes et al. on this topic showed that hydrate formation depends on the number of potential contacts for both donors and acceptors - its sum and its difference - in a molecule, rather than a simple donor-acceptor ratio; and on the polarity of the surface of the molecule, rather than its weight. ${ }^{25}$ The validity of these hypotheses has been tested with the purpose of prediction of hydrate formation, although it has been found to be less convenient than comparison of excess free energies of mixture between water and the organic molecule. ${ }^{26}$

Van de Streek and Motherwell suggested a different approach, based on the comparison of the molecular volume of a molecule in a hydrate and an anhydrate structure. ${ }^{27}$ The study found that, generally, larger organic molecules tend to use water as a space filler, based on the fact that the molecular volume for such molecules is larger in the anhydrate than in the hydrate. The same observation had already been made on the basis of molecular shape and hydration number. ${ }^{28}$ Molecular volumes for smaller molecules tend to be larger in the hydrate, meaning that water is incorporated in the structure for its H-bonding capabilities, causing a loss of close packing. Other factors, such as molecular flexibility or number of H-bond donors and acceptors, were also explored, but the authors recognised that none of the measured parameters have robust predictive capabilities.

In this chapter, volumetric differences between hydrates and anhydrates are analysed for hydrates which have been studied at high pressure. Examples of such analysis already exist in the literature ${ }^{29-32}$ but here a new rationale to determine pressure-dependent volumes for water molecules is explored and the study is extended to all high-pressure 
hydrates present in the CSD. This study stems from the observation that hydrate formation at high pressure is somewhat counterintuitive: on the one hand high pressure promotes efficient packing arrangements, which minimise molecular volumes, while on the other small molecules in hydrates generally exhibit larger molecular volumes than in the corresponding anhydrates. Analysing these differences can expose trends that can lead to a better understanding of hydrate crystal structures and hence be useful for subsequent CSP studies.

\subsection{Experimental}

\subsubsection{List of structures determined at high pressure}

The following terms are widely used in this text and require a clear definition:

- Determination: any ambient- or high-pressure (AP or HP) entry in the CSD with no restrictions in temperature.

- Structural set: group of entries with the same chemical composition. A structural set contains all determinations of all polymorphs of a substance, regardless of the pressure.

- Redetermination: any entry of a given structure having the same chemical composition, irrespective of the temperature and pressure at which it was measured.

- Main component: all the chemical entities in the chemical formula of a structure, except for water molecules.

- Anhydrate-hydrate (A-H) set: super-group of structures with a common main component and any variable number of water molecules.

A search of the CSD (v5.36 with updates to May 2015) was performed to find all the A-H sets of organic molecular substances which include at least one HP determination.

First, all HP entries were extracted from the CSD. The program Conquest (v1.17) ${ }^{33}$ was used to find all entries containing information in the pressure field, limiting the search to organic substances and 
entries free of errors. This search returns not only HP entries, but also $\mathrm{AP}$ ones for which a value of $0.0001 \mathrm{GPa}$ has been given in the pressure field. Those entries were removed. The query field organic limits the output to entries which do not contain transition metals, although it includes structures containing alkaline and alkaline earth metals. Additional search criteria were added to remove such structures from the output, because they tend to form aquo-complexes rather than hydrates.

Second, each determination was exchanged by the structural set in which it is contained. The structural formula of the main component of each determination was used as a search query. The resulting list contained, for each substance found in the first step, all the AP and HP determinations of its anhydrate and hydrate forms. Such search also yielded cocrystals, salts, and solvates of the main component, which were removed, unless an A-H set existed for the cocrystal, salt, or solvate. Stereoisomerism plays an important role in crystal packing, hence isomers of the same main component were always considered to form independent structural sets.

Determinations fulfilling any of the following criteria were removed from the list, as they were not suitable for this study:

- Hydration number not reported.

- Deuterated structures. Accounting for isotope effects, which are known to cause volume changes, ${ }^{34}$ was out of the scope of this study.

- No pressure value stated, instead simply determined at high pressure stated in the pressure field.

- Wrong or uncertain chemical formula, unit-cell volume, number of molecules per unit cell, space group, temperature, or pressure. The correctness of uncertain structures was checked in a case-by-case basis by referring to the original publication.

For the purpose of this study, the presence of deposited 3D coordinates was not a requirement, and a determination was deemed to be acceptable provided its unit-cell volume and number of chemical formulas per unit cell were reported. Nevertheless, structural sets for which no determina- 
tion has deposited 3D coordinates were removed, as it was not possible to check for potential errors. Additionally, an A-H set was removed entirely if, after applying the previous criteria, all the determinations of the hydrate forms or all the HP determinations were removed.

The final list contained 1602 entries divided among 194 structural sets. Of those sets, 55 corresponded to the 23 substances which form hydrates (Appx. A). The list of A-H sets can be further divided into three sub-lists (Appx. B):

- In situ-formed hydrates: sets in which at least one of the hydrates has been reported to form at high pressure. This list contains 7 sets.

- Compressed hydrates: sets in which hydrates existing at ambient pressure have been subject of compression studies. This list contains 6 sets.

- AP hydrates: sets for which the anhydrate, but not the hydrates, has been studied under pressure. This list contains 10 sets.

Three other structures needed to be added manually to the list of in situformed hydrates: ${ }^{t} \mathrm{BA}$ (reported in Ch. 6), parabanic acid (the pressure of the determinations was not recorded in the CSD, but on the corresponding publication ${ }^{35}$ ), and 5,6-dimethylbenzimidazole (dMBzIm, published ${ }^{32}$ although not available in the version of the CSD used).

\subsubsection{Compressibility of lattice water}

A new list was compiled from the hydrates, at either AP or HP, contained in the lists of in situ-formed hydrates and compressed hydrates. Molecular volumes were calculated as the volume within the Hirshfeld surface of a molecule $\left(V_{H}\right)$. A Hirshfeld surface defines the region around a molecule inside which the electron density of the molecule exceeds that of any neighbouring molecule. ${ }^{36}$ For each determination, $V_{H}$ of each entity of the chemical formula was computed using CRYstalExplorer. ${ }^{37}$ All $V_{H}$ were normalised to $293 \mathrm{~K}$ - the most frequent temperature recorded 
for HP determinations - using Hofmann's model: ${ }^{38}$

$$
V_{H}(293)=V_{H}(T)[1-\bar{\alpha}(T-293)]
$$

Where $V_{H}(293)$ is the estimated molecular volume at $293 \mathrm{~K} ; V_{H}(T)$ is the molecular volume at the temperature $T$ of the determination; and $\bar{\alpha}$ is the average thermal expansion coefficient determined by Hofmann, $0.95 \pm 0.03 \cdot 10^{-4} \mathrm{~K}^{-1}$.

$V_{H}$ were employed as a fast and reliable way to partition the unit-cell volume among all the chemical constituents of the crystal, albeit due to the nature of the Hirshfeld surface partitioning scheme, a small portion of the unit-cell volume remains unattributed (see §2.3.2 for further discussion). The unattributed volume of each determination $\left(V_{H, n t t}\right)$ was calculated as:

$$
V_{H, n t t}=V_{Z}-\sum V_{H, n w a t}-\sum V_{H, w a t}
$$

Where $V_{Z}$ is the volume of a chemical formula, obtained by dividing the unit-cell volume by $Z ; V_{H, \text { nwat }}$ is the molecular volume of a molecule other than water; and $V_{H \text {,wat }}$ is the molecular volume of a lattice water molecule. $V_{H, n t t}$ was used to estimate the significance level during the analysis of the results $(\S 2.3 .2)$.

$V_{H}$ of lattice water molecules were plotted in a $P V$ diagram and a Murnaghan equation of state ${ }^{39}$ was fitted to the data using EOSFIT7: ${ }^{40}$

$$
V_{H}=V_{H 0}\left(1+\frac{B_{0}^{\prime}}{B_{0}} P\right)^{-1 / B_{0}^{\prime}}
$$

Where $V_{H}$ is the molecular volume of a lattice water molecule at a pressure $P ; V_{H 0}$ is the corresponding volume at ambient pressure; $B_{0}$ is the bulk modulus at ambient pressure, in GPa; and $B_{0}^{\prime}$ is the pressure derivative of $B$. Only determinations below $5 \mathrm{GPa}$ were used, as this equation does not properly reproduce the compression of lattice water at higher pressures (§2.3.1). The fitted equation was later used to estimate the volume of lattice water at any pressure. 


\subsubsection{Pressure-volume diagrams of anhydrate-hydrate sets}

For each A-H set, all the possible phases were identified, and each determination assigned to its corresponding phase. For each determination, the molecular volume excluding water $\left(V_{\text {excl }}\right)$ was calculated. For hydrates, it is calculated as:

$$
V_{e x c l}=V_{Z}-N V_{H, w a t}
$$

Where $N$ is the number of water molecules in a chemical formula and $V_{H, w a t}$ is the molecular volume of a water molecule at the pressure of the determination, obtained from the fitted Murnaghan equation of state. For anhydrates, this volume corresponds simply to $V_{Z}$.

When several redeterminations of the same phase at the same pressure existed, a representative one was chosen according to the following criteria, in the stated order: ${ }^{41}$

- If there are four or more redeterminations, $V_{\text {excl }}$ of the representative structure must not differ considerably from the median $V_{\text {excl }}$ of all the redeterminations. This filters out redeterminations with poorly determined unit-cell parameters. If there are less than four redeterminations, it is not possible to ensure that $V_{\text {excl }}$ is free from errors.

- Redeterminations with deposited 3D coordinates are preferred, as they are helpful when checking for errors in the structure. Whenever a phase has a single determination, it is accepted even if no 3D coordinates are available.

- Determinations at room temperature are preferred over those at low temperature, as most HP structures are determined at $293 \mathrm{~K}$. This limits the errors introduced by the temperature normalisation.

- The redetermination with the lowest $R$ factor is preferred. 


\subsection{Results and discussion}

\subsubsection{Compressibility of lattice water}

Fig. 2.1 shows the compressibility of lattice water molecules in organic crystals, measured by means of Hirshfeld surfaces. Numerical data are provided in Appx. B. A Murnaghan equation (Eq. 2.3) was fitted to the experimental data from the CSD, obtaining the following parameters:

- $V_{H 0}=22.37 \AA^{3}$

- $B_{0}=7.92 \mathrm{GPa}$

- $B_{0}^{\prime}=3.91$

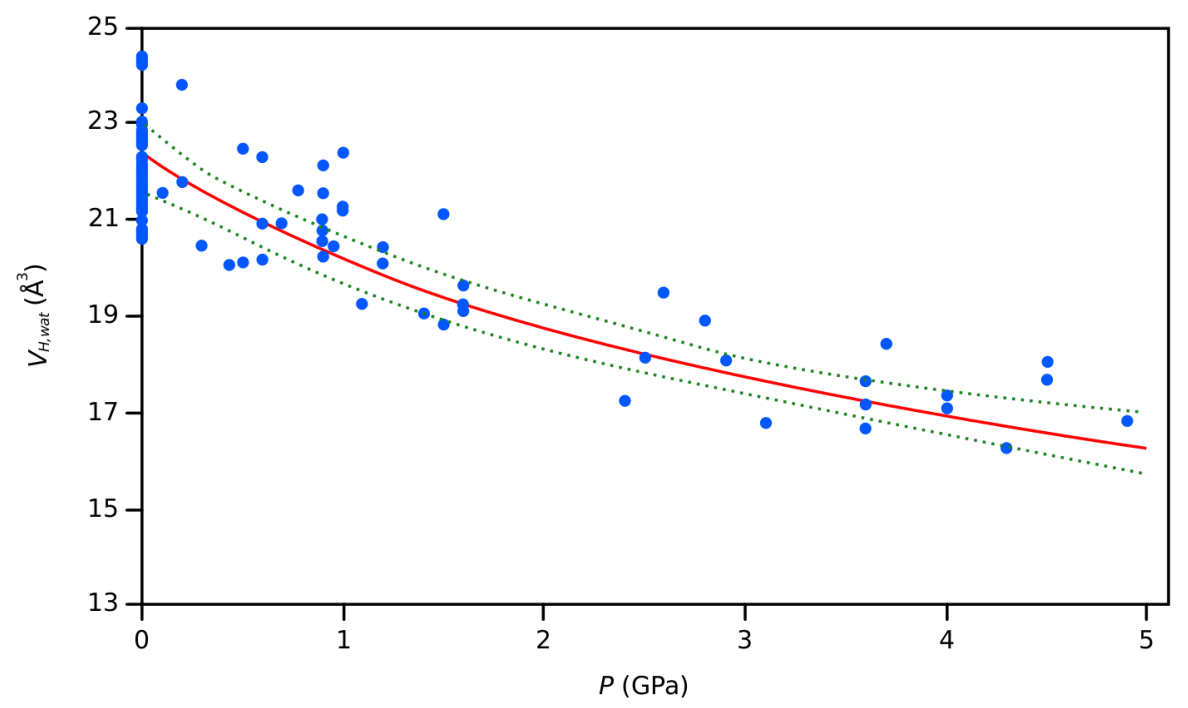

Figure 2.1: Compressibility of lattice water molecules in organic crystals, up to $5 \mathrm{GPa}$. The blue spots correspond to experimental molecular volumes within Hirshfeld surfaces. The red curve is the Murnaghan equation of state fitted to the data. The two green dotted curves correspond to the maximum and minimum uncertainties of the fit at the $2 \sigma$ level.

The Murnaghan equation of state is used for its simplicity, although it is deemed appropriate only to model small compressions for which $V / V_{H 0}>0.9 .^{42}$ For larger compressions the value of the fitted parameters $\left(V_{H 0}, B_{0}\right.$, and $\left.B_{0}^{\prime}\right)$ might not be representative of the physical properties of the system. For the compression of lattice water such condition is only fulfilled up to $2 \mathrm{GPa}$, with $V / V_{H 0} \approx 0.75$ at $5 \mathrm{GPa}$. The aim of this study is to provide a simple, yet reasonably accurate, way of estimating the volume of a lattice water molecule at a certain pressure, for which an equation which provides a good fit to the experimental data is more important than the physical meaning of its parameters. It 
has been considered that, from a mere mathematical perspective, the fit up to $5 \mathrm{GPa}$ provides a better representation of the system. If the Murnaghan equation is fitted only to data below $2 \mathrm{GPa}$, a linear equation provides a considerably better fit. The average residual sums of squares (RSS) are 1.414 and 0.644, for the Murnaghan and linear fit respectively. The use of the Murnaghan equation is discouraged for such data, while the use of a linear fit misses the pressure dependence of the compressibility. A Murnaghan equation of state fitted to all data up to $5 \mathrm{GPa}$ exhibits an average RSS of 0.769 , acceptable if compared with that of the linear fit in the smaller pressure range. In addition, it allows to explore a larger range of pressures, a critical factor considering the limited number of available structures of hydrates at high pressure. Data over $5 \mathrm{GPa}$ have not been considered, as there are only a few determinations available and their addition results in a considerable loss of quality in the fit.

In previous studies, the molecular volume of water in the liquid state or in ice VI (below and above 1.0 GPa, respectively) has been used as the volume of lattice water in organic structures. ${ }^{29-32}$ Although a good approximation, it has been considered that the use of experimentally measured volumes, by means of Hirshfeld surfaces in this case, provides a more physically meaningful approach.

\subsubsection{Assessment of errors}

Several different sources of error can be identified in this study. Some of them originate from the structures in the CSD: accuracy of the refined structures, unit-cell errors, and uncertainties in the measurement of pressures. Other errors stem from the use of Hirshfeld surfaces to compute molecular volumes and the equation of state used to estimate the volume of water molecules at different pressures. A comprehensive error model has not been included, as the level of complexity required to address all the different sources combined is not justified given the limited amount of data employed in the study. Instead, each source of error is discussed in this section and a framework in which to analyse the results is set up. 
The first source of error are the structures taken from the CSD. Structures are checked and errors are usually addressed upon deposition in the database, although there can be small errors, like misplaced hydrogen atoms or poorly refined disorder, leading to inaccurate geometries, which would affect the calculation of the molecular volumes. The refinement and molecular geometry of each structure used in this study was not checked on a case-by-case basis. Mogul ${ }^{43}$ provides a tool that allows for quick comparison of the geometry of a structure against the rest of the CSD, but considerably user input is still required to analyse the results. A completely automated geometry check would be desirable to evaluate large datasets. After filtering potentially problematic structures according to the criteria established in $§ 2.2 .3$, remaining errors are assumed to be small. Similarly, possible errors in the reported unit-cell parameters and pressure values are also assumed to be small, as there is no possibility to perform independent checks on these.

The second source of error originates from the use of Hirshfeld surfaces. Hirshfeld's model attempts to partition the electron density of a molecule among all its atoms ${ }^{44}$ and has later been adapted to partition the electron density of a unit cell among all its molecules. ${ }^{45}$ For a given molecule, a Hirshfeld surface defines the region around it inside which the electron density of the molecule exceeds that of any neighbouring molecule. Such a surface guarantees maximum proximity to the neighbouring molecules without an overlap. ${ }^{36}$ Because Hirshfeld surfaces are based on the partition of the spherically averaged electron density, rather than the unit-cell volume, they do not achieve a full partition of the volume. It is estimated that about $5 \%$ of the unit-cell volume is not attributed to any molecule. ${ }^{46}$ As a result, only differences in $V_{\text {excl }}$ between the anhydrate and the hydrate which are larger than the unattributed volume can be considered significantly different. For the structures included in this study, the average $V_{H, n t t}$ is $7.01 \pm 0.41 \AA^{3}$ (at the $95 \%$ level of confidence).

An alternative scheme for the partition of the unit-cell volume has been proposed by Hofmann, ${ }^{38}$ although its application in this study was not satisfactory. Said model proposes the use of averaged atomic 
volumes, determined by directly partitioning the unit-cell volume over all atoms in the structure, across the entire CSD. This approach was used by Van de Streek, ${ }^{27}$ to directly compute the packing efficiency of hydrates when compared to anhydrates. However, convergence to the appropriate atomic volumes requires a very large experimental dataset - the volumes obtained by this method for infrequent elements are not accurate. The number of high-pressure structures available in the CSD, for instance, is too small to compute the pressure dependence of atomic volumes. Molecular volumes determined from the partition of the electron density are better suited for small datasets. Some models can produce a complete partition, without empty volume, as proposed in Quantum Theory of Atoms in Molecules, ${ }^{47}$ but require charge-density grade refinements, ${ }^{36,48}$ which usually are not readily available.

Table 2.1: Value, variance, covariance, and correlation coefficient of the parameters of the Murnaghan equation fitted to the data of the compression of lattice water.

\begin{tabular}{lr}
\hline $\boldsymbol{V}_{\mathbf{0}}$ & 22.37 \\
$\boldsymbol{B}_{\mathbf{0}}$ & 7.92 \\
$\boldsymbol{B}_{\mathbf{0}}^{\prime}$ & 3.91 \\
$\boldsymbol{V a r}\left(\boldsymbol{V}_{\mathbf{0}}\right)$ & 0.15 \\
$\boldsymbol{V a r}\left(\boldsymbol{B}_{\mathbf{0}}\right)$ & 6.88 \\
$\boldsymbol{V a r}\left(\boldsymbol{B}_{\mathbf{0}}^{\prime}\right)$ & 3.20 \\
$\boldsymbol{C o v}\left(\boldsymbol{V}_{\mathbf{0}}, \boldsymbol{B}_{\mathbf{0}}\right)$ & -0.71 \\
$\boldsymbol{C o v}\left(\boldsymbol{V}_{\mathbf{0}}, \boldsymbol{B}_{\mathbf{0}}^{\prime}\right)$ & 0.31 \\
$\boldsymbol{C o v}\left(\boldsymbol{B}_{\mathbf{0}}, \boldsymbol{B}_{\mathbf{0}}^{\prime}\right)$ & -4.34 \\
$\boldsymbol{C o r r}\left(\boldsymbol{V}_{\mathbf{0}}, \boldsymbol{B}_{\mathbf{0}}\right)$ & -0.71 \\
$\boldsymbol{C o r r}\left(\boldsymbol{V}_{\mathbf{0}}, \boldsymbol{B}_{\mathbf{0}}^{\prime}\right)$ & 0.46 \\
$\boldsymbol{C o r r}\left(\boldsymbol{B}_{\mathbf{0}}, \boldsymbol{B}_{\mathbf{0}}^{\prime}\right)$ & -0.92 \\
\hline
\end{tabular}

The third source of error corresponds to the estimation of the volume of the water molecules. The uncertainty on the estimated volumes, $\operatorname{Var}(V)$, is determined by error propagation on the Murnaghan equation (Eq. 2.3):

$$
\begin{gathered}
\operatorname{Var}\left(V_{H}\right)=\left(\frac{\partial V_{H}}{\partial V_{H 0}}\right)^{2} \operatorname{Var}\left(V_{H 0}\right)+\left(\frac{\partial V_{H}}{\partial B_{0}}\right)^{2} \operatorname{Var}\left(B_{0}\right)+ \\
\left(\frac{\partial V_{H}}{\partial B_{0}^{\prime}}\right)^{2} \operatorname{Var}\left(B_{0}^{\prime}\right)+\frac{\partial V_{H}}{\partial V_{H 0}} \frac{\partial V_{H}}{\partial B_{0}} \operatorname{Cov}\left(V_{H 0}, B_{0}\right)+ \\
\frac{\partial V_{H}}{\partial V_{H 0}} \frac{\partial V_{H}}{\partial B_{0}^{\prime}} \operatorname{Cov}\left(V_{H 0}, B_{0}^{\prime}\right)+\frac{\partial V_{H}}{\partial B_{0}} \frac{\partial V_{H}}{\partial B_{0}^{\prime}} \operatorname{Cov}\left(B_{0}, B_{0}^{\prime}\right)
\end{gathered}
$$

Where $\operatorname{Var}\left(V_{H 0}\right), \operatorname{Var}\left(B_{0}\right), \operatorname{Var}\left(B_{0}^{\prime}\right), \operatorname{Cov}\left(V_{H 0}\right), \operatorname{Cov}\left(B_{0}\right)$, and $\operatorname{Cov}\left(B_{0}^{\prime}\right)$ are the variances and covariances of the three parameters fitted by the equation (Table 2.1). The correlations between these parameters where measured as Pearson product-moment correlation coefficients (Eq. 2.6). Said correlations are -0.71 for the pair $V_{H 0}, B_{0} ; 0.46$ for $V_{H 0}, B_{0}^{\prime}$; and -0.92 for $B_{0}, B_{0}^{\prime}$. With such large correlations, the use of covariances (2.6) in the error propagation cannot be neglected. The partial derivatives needed for the propagation of uncertainties are as follows:

$$
\frac{\partial V_{H}}{\partial V_{H 0}}=\left(1+\frac{B_{0}^{\prime}}{B_{0}} P\right)^{-1 / B_{0}^{\prime}}
$$




$$
\begin{gathered}
\frac{\partial V_{H}}{\partial B_{0}}=\frac{V_{H 0} P}{B_{0}^{2}}\left(1+\frac{B_{0}^{\prime}}{B_{0}} P\right)^{\left(-1 / B_{0}^{\prime}\right)-1} \\
\frac{\partial V_{H}}{\partial B_{0}^{\prime}}=-\frac{V_{H 0} P}{B_{0} B_{0}^{\prime}}\left(1+\frac{B_{0}^{\prime}}{B_{0}} P\right)^{\left(-1 / B_{0}^{\prime}\right)-1}+ \\
\frac{V_{H 0}}{B_{0}^{\prime 2}}\left(1+\frac{B_{0}^{\prime}}{B_{0}} P\right)^{-1 / B_{0}^{\prime}} \ln \left(1+\frac{B_{0}^{\prime}}{B_{0}} P\right)
\end{gathered}
$$

Combining Eqs. 2.7, 2.8, and 2.9 in Eq. 2.5 the uncertainties can be computed in all the pressure range. Said uncertainties, at the $2 \sigma$ level (95\% of confidence), are plotted in Fig. 2.1 as dotted green lines. The maximum uncertainty in all the pressure range is less than $1 \AA^{3}$, hence the error originating from the volume estimation can be neglected if compared with that coming from the use of Hirshfeld surfaces.

The estimation of the volume of water molecules could be considered unnecessary, as such volumes can be measured directly for each crystal structure, avoiding the uncertainty associated with any fitting. However, there are three main reasons for proceeding with an estimation:

- The use of estimated volumes from a fit to 75 experimental observations is likely to compensate for isolated errors in certain structures, which may arise if a single experimental volume is used.

- $V_{H}$ calculated with CRYSTALExPLORER have no uncertainty associated with them. That is compensated by the use of $V_{H, n t t}$ and the uncertainty of the estimation from the fit to the Murnaghan equation.

- An equation of state for the compressibility of water in molecular crystals is interesting for its predictive power. This may find applications in CSP, for instance in the calibration of the intra- and intermolecular potentials. This study also serves as a test of the validity of such an equation of state.

\subsubsection{Pressure-volume diagrams of anhydrate-hydrate sets}

Fig. 2.2 contains the $P V$ diagrams for all in situ-formed and compressed hydrates. Numerical data corresponding to these diagrams are provided 
Figure 2.2: Comparison between the molecular volumes, measured as $V_{H}$, of the anhydrate forms (in orange, full icons, different polymorphs labelled in Roman numerals or Greek letters, as defined in the original literature) and the hydrate forms (in blue, empty icons, labelled by their hydration numbers) of different materials. For structures belonging to a compression study, volumes at different pressures are linked by a solid line. For hydrates, $V_{Z}$ and $V_{\text {excl }}$ are plotted (above and below, respectively), linked by vertical dotted lines.
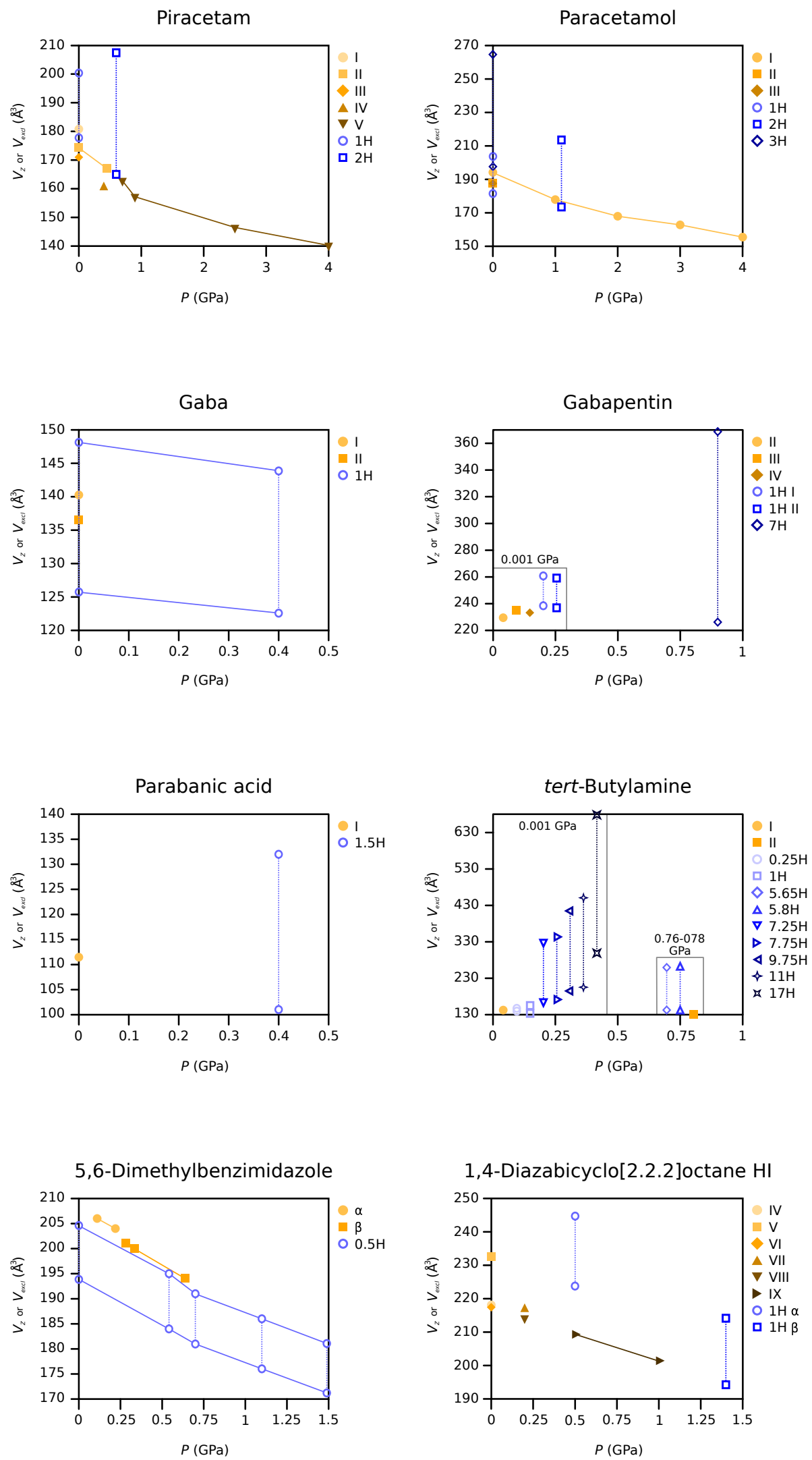

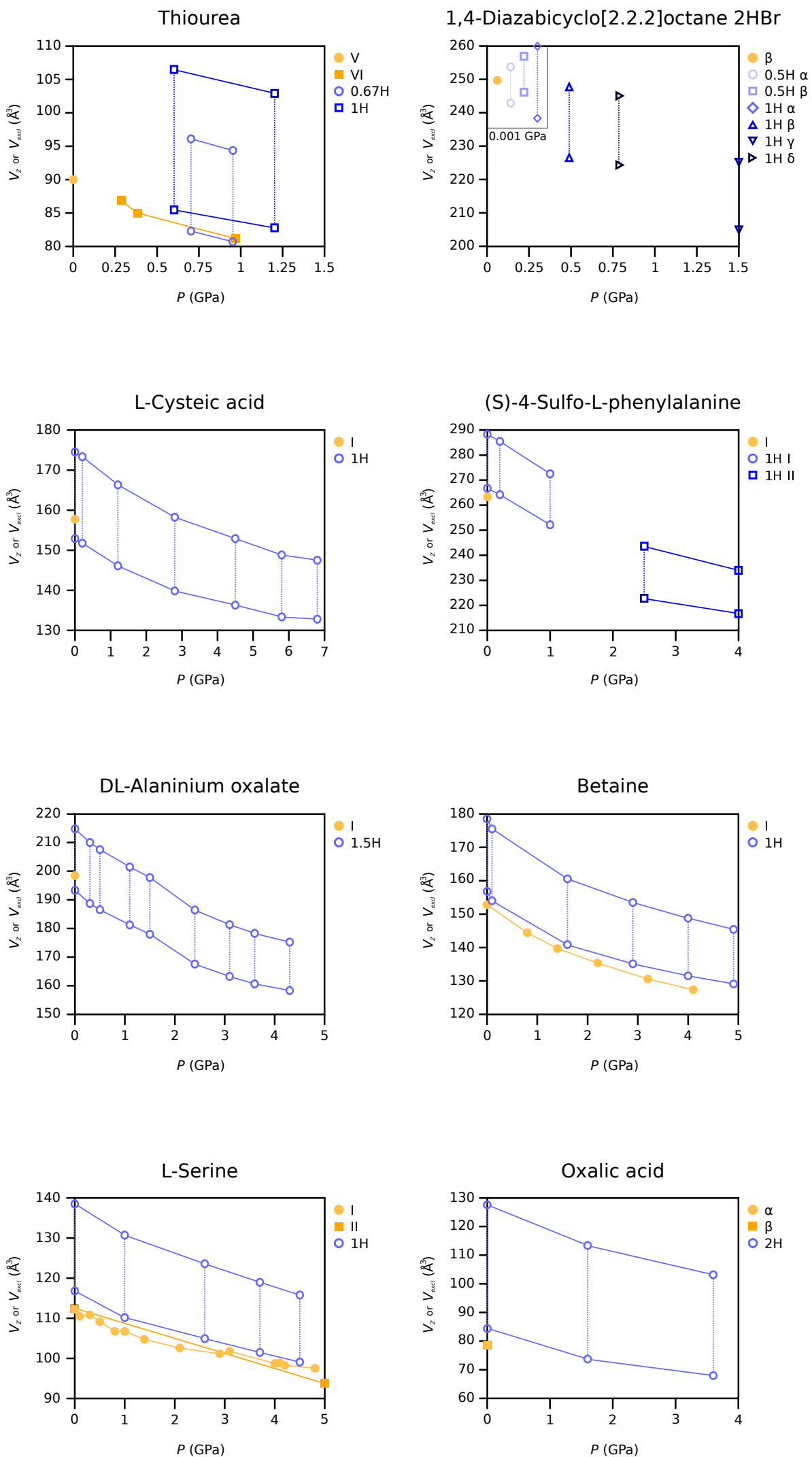
in Appx. C, were data from the group of AP hydrates are also provided. For hydrates, $V_{Z}$ and $V_{\text {excl }}$ are plotted, although only $V_{\text {excl }}$ should be compared with $V_{Z}$ of the anhydrate. $V_{Z}$ of the hydrates are given to provide a reference for the total volume of each substance.

The analysis of the plots yields two general remarks:

- $V_{Z}$ is generally larger for hydrates than for anhydrates. This was an expected result, as $V_{Z}$ of a hydrate includes the volume of the water molecules.

- For the majority of structures, both in situ-formed and compressed, $V_{\text {excl }}$ of a hydrate tends to be larger than $V_{Z}$ of an anhydrate, although differences are usually smaller than the significance level established in the previous section, $7.01 \pm 0.41 \AA^{3}$. The introduction of additional $\mathrm{H}$ bonds involving water molecules in the hydrate promotes a loss of close packing, which increases $V_{\text {excl }},{ }^{27}$ albeit this loss cannot be too large, otherwise the structure would probably not crystallise at high pressure in the first place.

The only case in this study in which both $V_{Z}$ and $V_{\text {excl }}$ of the hydrate are smaller than $V_{Z}$ of the anhydrate is dMBzIm. ${ }^{32}$ The hydrate of this compounds can be crystallised only at high pressure (although it can be recovered to ambient pressure and kept in such conditions for long periods of time). Zielinski et al. argue that this is not a case of special behaviour of the hydrate, but rather of the anhydrate, which exhibits a loosely packed structure. In this case, water can be incorporated into the structure in already-available space, resulting in a more efficient packing.

The opposite effect- $V_{\text {excl }}$ significantly larger for the hydrate at high pressure - is observed for 1,4-diazabicyclo[2.2.2] octane (dabcoHI) and ${ }^{t} \mathrm{BA}$. In the case of dabcoHI, ${ }^{30} V_{\text {excl }}$ for the $\alpha$ monohydrate is $14.4 \AA^{3}$ larger than that of phase IX at the same pressure. With such difference, the anhydrate may be expected to be the more favoured structure at high pressure. Two alternatives exist to explain the formation of the hydrate. First, the volume reduction still takes place during the experiment, although in the PTM (aqueous solution in this case) rather than in the crystal itself. This consideration may be generally relevant for materials which can form very dense solutions. Densities inside the DAC were 
not measured in the study. Second, the hydrate, unfavoured in terms of volume, is considerably more stable from an energy point of view, e.g. through the formation of a more efficient H-bonded network promoted at high pressure. According to the authors the latter is the most likely explanation, although no evidence was provided. Furthermore, kinetic effects can also play a role in the formation of the hydrate, but were not investigated

${ }^{t} \mathrm{BA}$ (Ch. 6) is the only clathrate among the structures considered in this study. The 17-hydrate, obtained at ambient pressure and low temperature, exhibits a $V_{\text {excl }}$ of $299.1 \AA^{3}$, twice larger than that of the anhydrate phase I. This is unsurprising, as all the small cages in the clathrate are empty, and the resulting space available is distributed over the guest molecules according to the partition scheme used herein (§2.3.2). The rest of the ambient-pressure ${ }^{t} \mathrm{BA}$ clathrates (7.25-, 7.75-, 9.75-, and 11-hydrate) behave in a similar manner. In contrast, $V_{\text {excl }}$ for the two high-pressure clathrates (5.65- and 5.8-hydrate) are only 12.2 and $9.6 \AA^{3}$ larger, respectively, than the volume of the anhydrate phase II, crystallised at a similar pressure. Although the difference in volume is larger than that found in most other examples, it is very small compared to the AP clathrates, exemplifying how efficient packing is enforced at high pressures.

For the remainder of the structures analysed, $V_{\text {excl }}$ of the hydrate is either smaller or not significantly larger than $V_{Z}$ of the anhydrate at similar pressures. Other interesting features might be identifiable; however, a larger number of structures would be necessary to attempt further analysis.

\subsection{Conclusions and outlook}

Crystallisation is a complex process that is influenced by many different factors. Packing efficiency and energy stabilisation are two of such factors which are commonly used to systematise crystallisation outcome. Except for materials with a substantial amount of void space in their anhydrous crystal structure, hydration tends to promote looser packings, 
which can be compensated with the formation of stabilising $\mathrm{H}$ bonds between organic molecules and lattice water. This study is focussed on the formation of hydrates at high pressure, conditions under which packing efficiency gains more importance, and proposes an analysis based on molecular volumes.

An equation of state for the compressibility of lattice water has been determined. Previous comparisons of the volumes of organic molecules in different hydration states have resorted to liquid water or ice VI to estimate the molecular volume of lattice water. In spite of the uncertainty associated to the fit, the approach proposed here is advantageous, as it is more likely to reflect the behaviour of water in crystals of organic structures.

The number of structures of hydrates at high pressure is still very limited, but most of the examples show that the volume of a formula unit in a crystal - after removing the volume occupied by water molecules - is smaller or not significantly larger than the same volume in an anhydrate phase at the same pressure. This may indicate that energy contributions can easily compensate any loss in packing efficiency caused by addition of water in the structure. Only three exceptions to this behaviour have been found. Both ${ }^{t} \mathrm{BA}$ and dabcoHI exhibit molecular volumes in the hydrate phases larger than in the anhydrate one. This effect can be easily explained in the case of ${ }^{t} \mathrm{BA}$ based on the formation of hydrate clathrates. The behaviour of dabcoHI poses a bigger challenge, and as for now there is not a satisfactory explanation for the formation of the HP hydrate. The opposite effect is observed in dMBzIm, where the hydrate is smaller than the anhydrate, even before removing the volume of water molecules. This is explained by a rather loose packing of the anhydrate.

Based on the results of this study, it would be desirable that all reports of high-pressure crystal structures include compression studies and molecular volumes measured by means of Hirshfeld surfaces, as to facilitate comparative studies. More experimental data will allow for a more comprehensive study, which in turn is likely to reveal more trends of hydrate formation at high pressure, from which structural chemistry and CSP can greatly benefit. Notwithstanding, volume considerations, 
although very important at high pressure, are only one of many contributions to hydrate formation. Further studies, considering lattice energies and kinetic effects, will also be needed to deepen our understanding of hydrate formation under high-pressure conditions. 


\section{References}

[1] S. L. Price, Chem. Soc. Rev., 2014, 43, 2098-2111.

[2] D. A. Bardwell, C. S. Adjiman, Y. A. Arnautova, E. Bartashevich, S. X. M. Boerrigter, D. E. Braun, A. J. Cruz-Cabeza, G. M. Day, R. G. Della Valle, G. R. Desiraju, B. P. Van Eijck, J. C. Facelli, M. B. Ferraro, D. Grillo, M. Habgood, D. W. M. Hofmann, F. Hofmann, K. V. J. Jose, P. G. Karamertzanis, A. V. Kazantsev, J. Kendrick, L. N. Kuleshova, F. J. J. Leusen, A. V. Maleev, A. J. Misquitta, S. Mohamed, R. J. Needs, M. A. Neumann, D. Nikylov, A. M. Orendt, R. Pal, C. C. Pantelides, C. J. Pickard, L. S. Price, S. L. Price, H. A. Scheraga, J. van de Streek, T. S. Thakur, S. Tiwari, E. Venuti and I. K. Zhitkov, Acta Cryst., 2011, B67, 535-551.

[3] J. Bernstein, Polymorphism in Molecular Crystals, Oxford Univ. Press, 2007.

[4] A. Y. Lee, D. Erdemir and A. S. Myerson, Annu. Rev. Chem. Biomol. Eng., 2011, 2, 259-280.

[5] A. J. Cruz-Cabeza, S. M. Reutzel-Edens and J. Bernstein, Chem. Soc. Rev., 2015, 44, 8619-8635.

[6] T. S. Thakur, R. Dubey and G. R. Desiraju, Annu. Rev. Phys. Chem., 2015, 66, 21-42.

[7] M. A. Neumann, J. van de Streek, F. P. A. Fabbiani, P. Hidber and O. Grassmann, Nat. Commun., 2015, 6, 7793.

[8] E. Gibney, Nature, 2015, 527, 20-21.

[9] B. P. van Eijck and J. Kroon, Acta Cryst., 2000, B56, 745.

[10] A. T. Hulme and S. L. Price, J. Chem. Theory Comput., 2007, 3, $1597-1608$.

[11] K. M. Anderson, G. M. Day, M. J. Paterson, P. Byrne, N. Clarke and J. W. Steed, Angew. Chem. Int. Ed., 2008, 47, 1058-1062.

[12] D. E. Braun, P. G. Karamertzanis and S. L. Price, Chem Comm, 2011, 47, 5443-5445.

[13] K. Takieddin, Y. Z. Khimyak and L. Fábián, Cryst. Growth Des., 2015, 16, 70-81. 
[14] D. W. M. Hofmann and J. Apostolakis, J. Mol. Struct., 2003, 647, 17-39.

[15] A. J. Cruz Cabeza, E. Pidcock, G. M. Day, W. D. S. Motherwell and W. Jones, CrystEngComm, 2007, 9, 556-560.

[16] J. Nyman and G. M. Day, CrystEngComm, 2015, 17, 5154-5165.

[17] J. D. Dunitz, ChemComm, 2003, 545-548.

[18] G. A. Jeffrey and H. Maluszynska, Acta Cryst., 1990, B46, 546-549.

[19] M. C. Etter, Acc. Chem. Res., 1990, 23, 120-126.

[20] G. R. Desiraju, J. Chem. Soc., Chem. Commun., 1991, 6, 426-428.

[21] L. Infantes and S. Motherwell, CrystEngComm, 2002, 4, 454-461.

[22] A. L. Gillon, N. Feeder, R. J. Davey and R. Storey, Cryst. Growth Des., 2003, 3, 663-673.

[23] L. Infantes, J. Chisholm and S. Motherwell, CrystEngComm, 2003, 5, 480-486.

[24] L. Infantes and W. D. S. Motherwell, ChemComm, 2004, 1166-1167.

[25] L. Infantes, L. Fábián and W. D. S. Motherwell, CrystEngComm, $2007, \mathbf{9}, 65-71$.

[26] Y. A. Abramov, CrystEngComm, 2015, 17, 5216-5224.

[27] J. van de Streek and S. Motherwell, CrystEngComm, 2007, 9, 55-64.

[28] C. H. Görbitz and H.-P. Hersleth, Acta Cryst., 2000, B56, 526-534.

[29] M. Andrzejewski, A. Olejniczak and A. Katrusiak, Cryst. Growth Des., 2011, 11, 4892-4899.

[30] A. Olejniczak and A. Katrusiak, Cryst. Growth Des., 2011, 11, $2250-2256$.

[31] H. Tomkowiak, A. Olejniczak and A. Katrusiak, Cryst. Growth Des., 2013, 13, 121-125.

[32] W. Zieliński and A. Katrusiak, CrystEngComm, 2015, 17, 54685473.

[33] I. J. Bruno, J. C. Cole, P. R. Edgington, M. Kessler, C. F. Macrae, P. McCabe, J. Pearson and R. Taylor, Acta Cryst., 2002, B58, 389397.

[34] V. S. Kogan, Sov. Phys. Usp., 1963, 5, 951-975.

[35] F. P. A. Fabbiani, D. R. Allan, W. G. Marshall, S. Parsons, C. R. Pulham and R. I. Smith, J. Cryst. Growth, 2005, 275, 185-192. 
[36] M. A. Spackman and D. Jayatilaka, CrystEngComm, 2009, 11, 1932 .

[37] S. Wolff, D. Grimwood, J. McKinnon, M. Turner, D. Jayatilaka and M. Spackman, CRYSTAL EXPLORER v3.1, University of Western Australia, 2012.

[38] D. W. M. Hofmann, Acta Cryst., 2002, B57, 489-493.

[39] F. Murnaghan, Am. J. Math., 1937, 49, 235-260.

[40] R. J. Angel, J. Gonzalez-Platas and M. Alvaro, Z. Kristallogr., 2014, 229, 405-419.

[41] J. van de Streek, Acta Cryst., 2006, B62, 567-579.

[42] R. J. Angel, High-Pressure Crystallography, Springer Science \& Bussiness Media, 2004, ch. 1.2, pp. 21-36.

[43] I. J. Bruno, J. C. Cole, M. Kessler, J. Luo, W. D. S. Motherwell, L. H. Purkis, B. R. Smith, R. Taylor, R. I. Cooper, S. E. Harris and A. G. Orpen, J. Chem. Inf. Comput. Sci., 2004, 44, 2133-2144.

[44] F. L. Hirshfeld, Theor. Chim. Acta, 1977, 44, 129-138.

[45] M. A. Spackman and P. G. Byrom, Chem. Phys. Lett., 1997, 267, $215-220$

[46] J. J. McKinnon, A. S. Mitchell and M. A. Spackman, Chem. Eur. J., 1998, 4, 2136-2141.

[47] R. Bader, Atoms in Molecules - A Quantum Theory, Oxford Univ. Press, 1990.

[48] C. Gatti, V. R. Saunders and C. Roetti, J. Chem. Phys., 1994, 101, 10686-10696. 
CHAPTER 3

\section{Hydrates of $\boldsymbol{\beta}$-cyclodextrin at high pressure}

\subsection{Introduction}

At ambient pressure, $\beta$-CD (Fig. 3.1) hydrates incorporate a variable and non-stoichiometric number of water molecules, ranging from 9.4 to 12.3. Despite their variable water content, all hydrates belong to the same polymorph, named form I in this work. The lattice parameters were first reported by Hamilton et al. in $1968^{1}$ to belong to space group $P 2_{1}$, $a=10.31(2) \AA, b=20.86(1) \AA, c=15.2(09) \AA$, and $\beta=109.29(15)^{\circ}$. The hydration number was estimated at twelve water molecules by density measurements. These observations were subsequently confirmed by X-ray single-crystal diffraction; ${ }^{2,3}$ the refined structure exhibited a large degree of disorder in the water molecules, and clearly indicated that the $\beta$-CD molecule adopts a round shape. Such observations evidenced that different CDs, namely $\alpha$ - and $\beta$-CD, behaved rather differently in the solid state. While $\alpha$-CD was known to adopt a round shape when forming 
inclusion complexes and a slightly distorted one in the hydrated state; ${ }^{4}$ $\beta-\mathrm{CD}$ was consistently characterised as round in crystals of either the hydrate or any inclusion compound. ${ }^{5,6}$ This difference suggested that complexation could take place via different mechanisms depending on the size of the CD. ${ }^{3}$ Water molecules in $\beta$-CD form I, particularly those located inside the cavity, were not only disordered, but also deemed to form an inefficient H-bonded network. A similar situation is encountered in hydrates of $\alpha$-CD, albeit it is partially compensated by the conformational change of the macrocycle. This is one of the reasons why CDs are such efficient hosts in the formation of inclusion complexes; they have a natural tendency to displace water from the interior of the cavity (in favour of another guest) to the intermolecular spaces, where it can contribute towards more efficient $\mathrm{H}$ bonds. ${ }^{2}$

Figure 3.1: Chemical diagram of $\beta$-CD (right) and an isolated glucose unit (left). Each atom is given a label $m_{-} n$, indicating that it is the $m^{\text {th }}$ atom of the $n^{\text {th }}$ glucose unit.
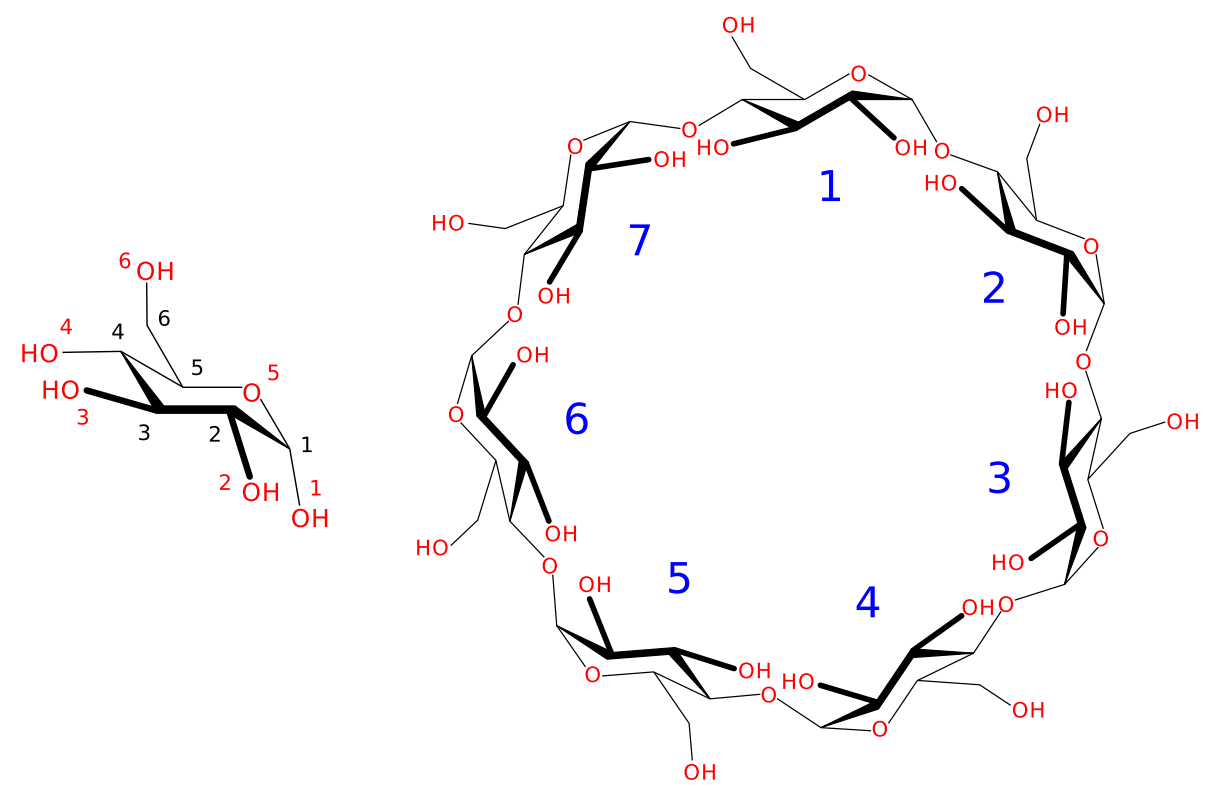

To further explore the behaviour of $\beta$-CD form I, a hydration study was carried out by Steiner and Koellner. ${ }^{7}$ It was found that $\beta$-CD undergoes a reversible dehydration process - from 12.3 to 9.4 water molecules, without a significant change in lattice constants - in response to changes in the humidity of the environment surrounding the crystal. The structure of the hydrate is based on a herringbone-type packing of $\beta$-CD molecules (Fig. 3.2), which lacks of channels that could allow water to circulate through the crystal. Based on packing consideration and 
neutron-diffraction observations, ${ }^{8}$ Steiner and coworkers postulated that the hydration/dehydration process could take place through an $\mathrm{H}$ bond flip-flop mechanism. In such mechanism, H-bonds flip between two configurations, causing a concerted chain reorganisation across the crystal able to alternatively open and close certain regions of the structure in a fast manner, moving water molecules along.

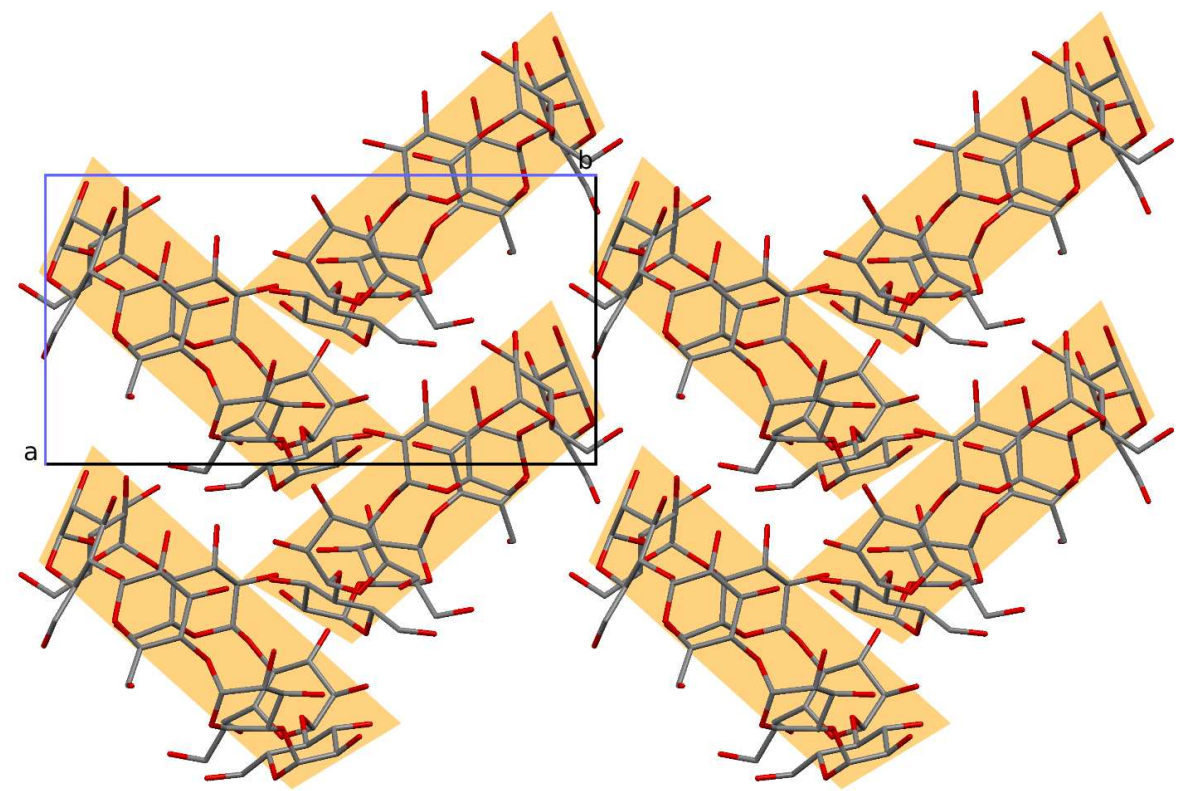

Figure 3.2: Herringbone packing of $\beta$-CD form I. Projection perpendicular to the $c$ axis. Trapeziums are used as a simplified way of representing the outline of projected $\beta$-CD molecules and to show the packing arrangement more clearly. $\mathrm{H}$ atoms, water molecules, and disorder have been omitted for clarity.

In this chapter, two new hydrates of $\beta$-CD, named forms II and III, crystallised in situ at high pressure, are reported. Both forms exhibit structural parameters and packing types that have already been described in the literature, although they are substantially different from the classic herringbone motif associated with hydrates and complexes incorporating small guests. ${ }^{9}$ The change in packing type can be linked to the super-hydration exhibited by both forms, a feature that seems to be characteristic of $\beta$-CD at high pressure. ${ }^{10}$ Super-hydration induced by pressure is known to occur in certain zeolites; ${ }^{11,12}$ it is also known that MOFs can accommodate large quantities of solvent under pressure. ${ }^{13}$ While hydrate and solvent formation of organic molecules can be enhanced under high pressure $(\S 1.1 .2), \beta-\mathrm{CD}$ is, to the best of our knowledge, the first example of a purely organic molecule that exhibits super-hydration. The concept of super-hydration is not unambiguously defined in the literature; hence for the scope of this work a super-hydrate 
is defined as a crystal structure that incorporates a substantially larger number of water molecules per formula unit, compared to other hydrates of the same molecule, and which cannot be described as a water clathrate or semi-clathrate.

Because of the high degree of disorder of the water network in forms II and III and the limitations imposed by the use of a DAC, the hydration numbers cannot be accurately determined. The present chapter elaborates on the determination of the water content and its implications on the crystallisation of forms II and III.

\section{$3.2 \quad$ Experimental}

\subsubsection{Crystal growth}

Hydrates of $\beta$-CD were produced by following the in situ high-pressure crystallisation procedure outlined in $§ 1.1 .2$. Several crystallisation experiments were performed and three crystals (named $\mathbf{A}, \mathbf{B}$ and $\mathbf{C}$ ) were ultimately measured by X-ray diffraction. Crystals $\mathbf{A}$ and $\mathbf{B}$ corresponded to form II at 0.93 and $0.92 \mathrm{GPa}$, respectively, while crystal $\mathbf{C}$ corresponded to form III at $0.72 \mathrm{GPa}$.

Single crystals of form I were produced from an aqueous saturated solution of $\beta$-CD, prepared by dissolving $\sim 40 \mathrm{mg}$ of the compound (Sigma-Aldrich, used as received; solubility at $298 \mathrm{~K}$ is $18.5 \mathrm{mg} / \mathrm{mL}$ ) in $\sim 1 \mathrm{~mL}$ of hot $(\sim 343 \mathrm{~K})$ demineralised water and then cooling down to room temperature. Subsequent to crystallisation, both crystals and the solution were employed for the high-pressure experiments.

Crystal $\mathbf{A}$ was grown in a square DAC (Appx. I.1) equipped with an Inconel 718 gasket $250 \mu \mathrm{m}$ thick pre-indented to a thickness of $170 \mu \mathrm{m}$ and with a hole $300 \mu \mathrm{m}$ in diameter. Given the small size of the sample chamber in the DAC and the relatively low solubility of $\beta$-CD (if compared with other $\mathrm{CDs}$ ), the amount of $\beta$-CD present in the chamber when loading the DAC with the solution only was not sufficient to grow a crystal of a suitable size for X-ray diffraction. This problem was overcome thanks to the inverse solubility of form I with pressure; that is, crystals dissolve, rather than grow, upon increasing pressure. A cer- 
tain amount of crystals of form I were loaded in the cell along with the saturated aqueous solution (Fig. 3.3a). Pressure increase resulted in the dissolution of the crystals, in turn increasing the amount of material in solution (Fig. 3.3b-e). Further increase in pressure produced a new crystalline phase - form II-above 0.90 GPa (pressure value depending on amount of $\beta$-CD inside the DAC).

Experimental observations showed that form II tends to grow polycrystalline (Fig. 3.3f) and that it is very insoluble at the pressure of crystallisation. Because of this, obtaining a single crystal of form II from its polycrystalline precipitate by temperature cycling is impractical, as it requires very high temperatures. In addition, it was observed that the dissolution of the polycrystalline form II always left residual particles in the sample chamber (Fig. 3.3g-i), which acted as nucleation points during recrystallisation on cooling, leading inevitably to the growth of several crystals. The origin of the residue is unknown, although it is suspected that, due to its excellent complex-formation capabilities, commercial $\beta$-CD might contain impurities which precipitate at high pressure. The amount of residue was always very small and difficult to recover once the DAC was opened, hence identification of its chemical composition was not possible. Based on these observations, it was concluded that polycrystalline precipitation of form II should be avoided.

The procedure finally used to grow crystal A consisted in increasing pressure gradually while monitoring the dissolution of the crystals loaded at the beginning of the experiment. Above 0.60 GPa, pressure was further increased in less than 0.05 GPa steps and the DAC was left to equilibrate for several hours in between each step. At $0.93 \mathrm{GPa}$, the crystals of form I dissolved completely and form II grew as a single crystal, which was measured by X-ray diffraction (Fig. 3.3j). A precise relationship of pressure steps and waiting times cannot be given, because it depends on the total amount of $\beta$-CD present in the sample chamber, which was not quantified, and the temperature at which the experiment is conducted (293-298 $\mathrm{K}$ in this case).

The other two crystals were grown following a similar procedure. Crystal B was grown in a triangular DAC (Appx. I.2) equipped with 
Figure 3.3: Crystallisation of $\beta$-CD form II (Crystal A). a) DAC loaded with crystals of form I and saturated solution. b-e) Dissolution as pressure increases. f) Crystallisation of form II in polycrystalline form. $\mathbf{g}, \mathbf{h})$ Isobaric dissolution of the crystals of form II upon heating. i) Residue left after the dissolution of the polycrystalline form II. j) Single crystal of form II at $0.93 \mathrm{GPa}$.
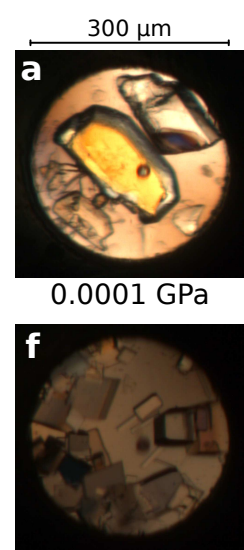

$0.90 \mathrm{GPa}$

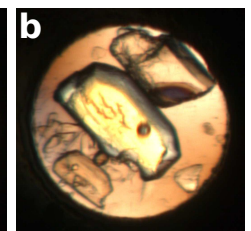

$0.22 \mathrm{GPa}$

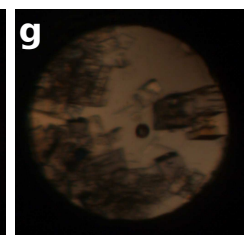

$0.90 \mathrm{GPa}$

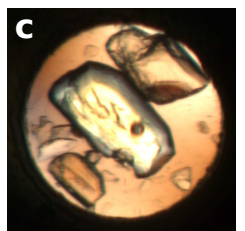

$0.40 \mathrm{GPa}$

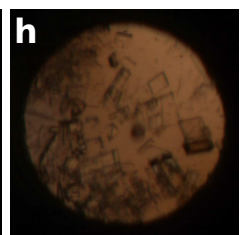

$0.90 \mathrm{GPa}$

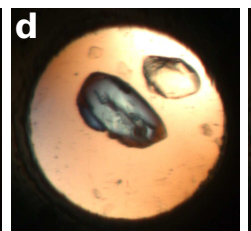

$0.68 \mathrm{GPa}$

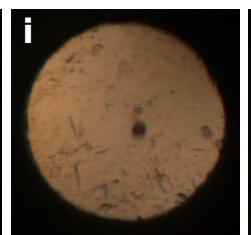

$0.90 \mathrm{GPa}$

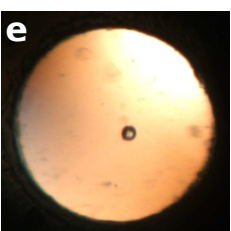

$0.81 \mathrm{GPa}$

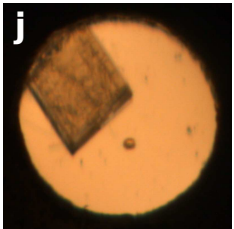

$0.93 \mathrm{GPa}$

a $200 \mu \mathrm{m}$ thick gasket pre-indented to $160 \mu \mathrm{m}$ with a hole $320 \mu \mathrm{m}$ in diameter. This crystal was grown and measured at 0.92 GPa (Fig. 3.4a). Crystal $\mathbf{C}$ was prepared in a 90/100 DAC (see Appx. I.3 for a technical description of the cell and further information on its use) with gasket of thickness $200 \mu \mathrm{m}$ pre-indented to $120 \mu \mathrm{m}$ and with a hole $270 \mu \mathrm{m}$ in diameter. This crystal (Fig. 3.4b), obtained and measured at $0.72 \mathrm{GPa}$, turned out to be a different HP phase - form III - despite not showing any optical difference (colour, morphology, etc.) with respect to form II.

Figure 3.4: a) Single crystal of $\beta$-CD form II at 0.92 GPa. b) Single crystal of $\beta$-CD form III at $0.72 \mathrm{GPa}$.
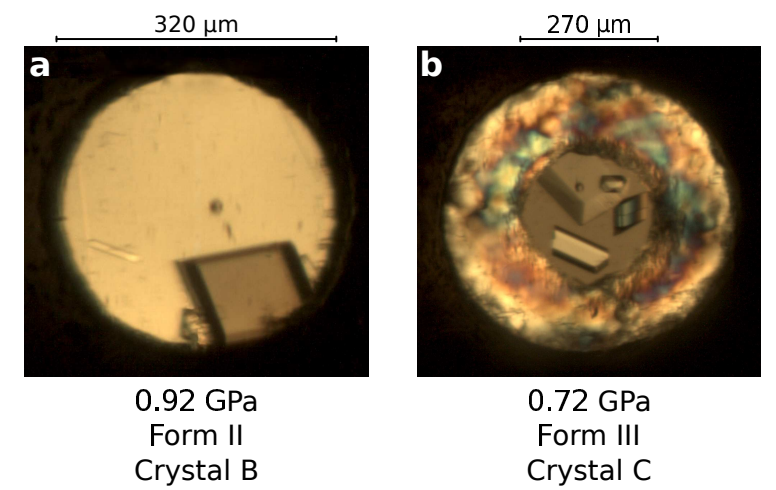

\subsubsection{X-ray data collection and processing}

Data on crystal A were collected at beamline F1 at Doris (DESY) on a Huber four-circle diffractometer, with $1^{\circ} \phi$ scans at two different values of $\chi\left(0\right.$ and $\left.90^{\circ}\right)$. Exposure time was $80 \mathrm{~s}$. Radiations of 0.5 and $0.65 \AA$ with a beam size of $1 \times 1 \mathrm{~mm}$ was used. Sample-detector distances were 200 and $130 \mathrm{~cm}$ for diffraction with 0.5 and $0.65 \AA$ radia- 
tion, respectively. The detector was a MAR165 CCD. Before integration, information in frame headers was updated with $\mathrm{CMH}$ and frames were converted from MAR to Bruker-Nonius Smart format using M2B. ${ }^{14}$ The frames were finally converted into SFRM format using the tools included in Apex2. ${ }^{15}$

Crystals $\mathbf{B}$ and $\mathbf{C}$ were measured at the P02.2 beamline at PetraIII (DESY) on a one-circle diffractometer. A $5 \times 2 \mu \mathrm{m}$ beam of $0.28962 \AA$ wavelength was used. The collection strategy consisted of $1^{\circ} \phi$ scans with the DACs mounted on the goniometer head in three different orientations $\left(60^{\circ}\right.$ rotations around the direction of the beam). Exposure time was $1 \mathrm{~s}$. Two detectors were used for these experiments: a MAR345 image plate and a Perkin-Elmer XRD 1621 amorphous-silicon flat panel. MAR frames were converted to SFRM format directly in APEX2. ESPERANTO frames from the Perkin-Elmer detector were converted into MAR format first using CrysAlis Pro, ${ }^{16}$ and then to SFrm format in Apex2.

All data were integrated following the strategy outlined in $§ 1.1 .3$.

\subsubsection{Structure refinement}

The structure of form II, determined from crystal A, was at first considered a pseudo-merohedral monoclinic twin on the basis of unexpectedly high $R$ factors. The data could be integrated in two possible monoclinic lattices, $P$ and $C$, both resembling an orthorhombic $C$ lattice, due to $a \approx c$ in the first case and $\beta \approx 90^{\circ}$ in the second. Due to the limited data available, it was not possible to elucidate the correct symmetry and the structure could be refined in space groups $P 2_{1}, C 2$, or $C 222_{1}$. Maximum resolution of the data was only $1.4 \AA$ with a completeness of $44 \%$ and a redundancy of 1.2 in space group $P 2_{1}$ and 2 in $C 2$. Crystal $\mathbf{B}$ provided data with a resolution up to $1.1 \AA$, which was sufficient to compare the refinement in the three possible space groups and determine that the orthorhombic one provides the best refinement. Data from crystals A and $\mathbf{B}$ were not merged. See discussion in §3.3.1 for more information.

Both forms II and III, from crystals $\mathbf{B}$ and $\mathbf{C}$ respectively, were refined following the same strategy. Models of $\beta$-CD molecules, taken from structures with the same lattice constants and space groups, were used 
as a starting point. The model for form II was provided by Saouane, ${ }^{10}$ while the model for form III corresponds to the structure deposited as WISREV $^{17}$ in the CSD. Full-matrix least-squares refinement against $F^{2}$ was performed in SHELXL v2014/ $7,{ }^{18}$ using the GUI SHELXLE. ${ }^{19}$ All ordered non-H atoms of the CD molecules were refined anisotropically, with their atomic displacement parameters (ADPs) subject to similarity restraints. Disordered atoms of the CD molecules - the primary hydroxy group (O6) in some of the glucose units - and all the water molecules were refined isotropically. Restraints were applied to all bonds, angles and torsion angles, with the exception of the torsion angles involving the atom $\mathrm{O6}$, which is known to be conformationally flexible. Restraints were automatically generated on the GRADE WeB SERVER. ${ }^{20}$ In addition, the geometry of all the glucose rings was restrained to be similar. The large number of restraints helps to compensate for the low data-to-parameter ratio of the high-pressure data and is justified on the basis that $\beta$-CD molecules exhibit the same conformation in all their structures - apart from the orientation of the $\mathrm{O} 6$ groups - hence the restraints do not bias the model. ${ }^{5,6} \mathrm{H}$ atoms of the $\beta$-CD molecule were placed geometrically and constrained using the riding model. No $\mathrm{H}$ atoms have been refined for water molecules. For further crystallographic data see Table 3.1.

An important consideration during the refinement of $\beta$-CD hydrates is the placement of water molecules. When considering the large solventaccessible volume in their structures and the unexpectedly low density for materials at 0.92 and $0.72 \mathrm{GPa}$, both forms II and III appear to contain a large amount of disordered water molecules, considerably larger than form I, which cannot be completely refined due to their extensive disorder and the limitations of high-pressure data. Refinement of disordered solvent is likely to cause a decrease in the $R$ factor, due to the increase in the number of refined parameters, ${ }^{22}$ even when the atomic positions do not match the electron density. To avoid over-fitting of the refined model, macromolecular crystallography (which faces problems similar to those of the high-pressure samples in this study: low-resolution and large solvent volume) makes use of the $R_{\text {free }}$ factor. ${ }^{23}$ 


\begin{tabular}{|c|c|c|}
\hline & Form II & Form III \\
\hline \multicolumn{3}{|l|}{ Crystal data } \\
\hline Chemical formula & $\mathrm{C}_{42} \mathrm{H}_{70} \mathrm{O}_{35} \cdot 25.5 \mathrm{H}_{2} \mathrm{O}$ & $\mathrm{C}_{42} \mathrm{H}_{70} \mathrm{O}_{35} \cdot 16.25 \mathrm{H}_{2} \mathrm{O}$ \\
\hline Molecular mass $\left(\mathrm{g} \mathrm{mol}^{-1}\right)$ & 1594.38 & 1427.73 \\
\hline Temperature $(\mathrm{K})$ & $298(2)$ & $298(2)$ \\
\hline Pressure (GPa) & $0.92(5)$ & $0.72(5)$ \\
\hline Crystal system & orthorhombic & triclinic \\
\hline Space group & $C 222_{1}$ & $P 1$ \\
\hline$a(\AA)$ & $19.7458(6)$ & $17.8952(19)$ \\
\hline$b(\AA)$ & $24.0901(6)$ & $15.4889(12)$ \\
\hline$c(\AA)$ & $32.980(5)$ & $15.5473(12)$ \\
\hline$\alpha\left({ }^{\circ}\right)$ & & $101.061(7)$ \\
\hline$\beta\left(^{\circ}\right)$ & & $113.078(9)$ \\
\hline$\gamma\left({ }^{\circ}\right)$ & & $101.440(8)$ \\
\hline$V\left(\AA^{3}\right)$ & $15688(2)$ & $3708.0(6)$ \\
\hline$Z$ & 8 & 8 \\
\hline Crystal size (mm) & $\sim 0.11 \times 0.10 \times 0.06$ & $\sim 0.09 \times 0.07 \times 0.05$ \\
\hline \multicolumn{3}{|l|}{ Data collection } \\
\hline Radiation wavelength $(\AA)$ & 0.28962 & 0.28962 \\
\hline Absorption correction & \multicolumn{2}{|c|}{ Semi-empirical (using $I$ meas.), SADABS ${ }^{21}$} \\
\hline$T_{\min }, T_{\max }$ & $0.8811,1.0000$ & $0.9586,1.0000$ \\
\hline Number of $[I>2 \sigma(I)]$ reflections: & & \\
\hline - measured & 29976 & 12992 \\
\hline - independent & 3977 & 7684 \\
\hline - observed & 3781 & 5113 \\
\hline Completeness (\%) & 64.7 & 73.2 \\
\hline$(\sin \theta / \lambda)_{\max }\left(\AA^{-1}\right)$ & 0.45 & 0.45 \\
\hline$R_{\text {int }}(\%)$ & 4.35 & 8.26 \\
\hline \multicolumn{3}{|l|}{ Refinement } \\
\hline$R\left[F^{2}>2 \sigma\left(F^{2}\right)\right](\%)$ & 9.46 & 12.82 \\
\hline$w R\left(F^{2}\right)(\%)$ & 24.85 & 32.06 \\
\hline$S$ & 1.203 & 1.112 \\
\hline Number of reflections & 3977 & 7684 \\
\hline Number of parameters & 876 & 1583 \\
\hline Number of restraints & 2194 & 5313 \\
\hline$\Delta \rho_{\min }, \Delta \rho_{\max }\left(\mathrm{e} \AA^{-3}\right)$ & $-0.390,0.492$ & $-0.443,0.510$ \\
\hline
\end{tabular}

Table 3.1: Crystallographic data and refinement parameters of $\beta$-CD forms II and III.

The $R$ factor is defined as:

$$
R=\frac{\sum_{h, k, l}\left\|F_{o b s}(h, k, l)-F_{c a l c}(h, k, l)\right\|}{\sum_{h, k, l}\left|F_{o b s}(h, k, l)\right|}
$$

Where $F_{o b s}(h, k, l)$ and $F_{c a l c}(h, k, l)$ are the observed and calculated structure factor amplitudes, respectively. $R_{\text {free }}$ is defined in the exact same way, but is computed using a set of reflections which are not used in the refinement. The rest of the reflections, against which the model is refined, allow to compute $R_{w o r k}$, which is equivalent to $R$.

For the purpose of this study, every tenth reflection has been moved to the $R_{\text {free }}$ set using XPREP. ${ }^{24}$ Refining in this way, only water molecules 
which provide a decrease in both $R_{\text {work }}$ and $R_{\text {free }}$ have been included in the model. An increase in $R_{\text {free }}$ after the introduction of certain water molecules is an indication that the electron density in the corresponding region is too smeared to model the water content. None of the $R$ factors changed significantly with the occupancies of the water molecules, which were set to either $25,50,75$, or $100 \%$ on the basis of the relative values of their isotropic ADPs.

This strategy was used until all possible water molecules were located. Then, several cycles of refinement against the full dataset were performed until convergence. This type of refinement evidences that the exact water content of forms II and III of $\beta$-CD cannot be determined by means of single-crystal X-ray diffraction. Several approaches to estimate the water content in this structures are discussed in the following section.

\subsection{Results and discussion}

\subsubsection{Twinning in $\beta$-cyclodextrin hydrate form II}

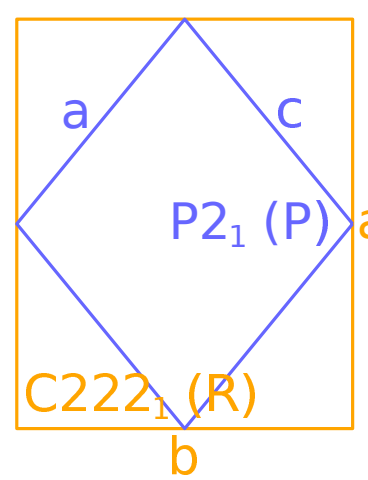

Figure 3.5: Relationship between the monoclinic $P 2_{1}$ (P setting, blue) and the orthorhombic $C 222_{1}$ (R setting, orange) unit cells of $\beta$-CD form II.

To address the possible existence of pseudo-merohedral twinning in the structure of $\beta$-CD form II, diffraction data were first indexed and integrated in the three possible settings (Table 3.2). The three settings are mutually related, as both space groups $P 2_{1}(\mathbf{P})$ and $C 2(\mathbf{Q})$ are subgroups of $C 222_{1}(\mathbf{R})$. Data statistics after integration, determined using XPREP, are summarised in Table 3.3. XPREP was also used to determine whether any of the monoclinic settings should be transformed into the orthorhombic unit cell (Table 3.4). The following matrices can be used to transform between the different settings (Fig. 3.5):

$$
\begin{array}{ll}
(\mathbf{P} \rightarrow \mathbf{R}) \quad P 2_{1} \rightarrow C 222_{1} & \left(\begin{array}{ccc}
1 & 0 & 1 \\
1 & 0 & -1 \\
0 & 1 & 0
\end{array}\right) \\
(\mathbf{Q} \rightarrow \mathbf{R}) \quad C 2 \rightarrow C 222_{1} & \left(\begin{array}{lll}
1 & 0 & 0 \\
0 & 1 & 0 \\
0 & 0 & 1
\end{array}\right)
\end{array}
$$


3.3. Results and discussion

\begin{tabular}{ccccccc}
\hline Setting & $\begin{array}{c}\text { Space } \\
\text { group }\end{array}$ & $\boldsymbol{a}(\AA)$ & $\boldsymbol{b}(\AA)$ & $\boldsymbol{c}(\AA)$ & $\boldsymbol{\beta}\left({ }^{\circ}\right)$ & $\boldsymbol{V}\left(\AA^{\mathbf{3}}\right)$ \\
\hline $\mathbf{P}$ & $P 2_{1}$ & $15.5933(4)$ & $32.995(4)$ & $15.5927(4)$ & $101.313(3)$ & $7866.6(10)$ \\
$\mathbf{Q}$ & $C 2$ & $19.7575(5)$ & $24.1014(7)$ & $32.983(5)$ & $90.013(5)$ & $15706(3)$ \\
$\mathbf{R}$ & $C 222_{1}$ & $19.7458(6)$ & $24.0901(6)$ & $32.980(5)$ & 90 & $15688(2)$ \\
\hline
\end{tabular}

Table 3.2: Possible settings for $\beta$-CD form II.

\begin{tabular}{cccccccc}
\hline Setting & $\begin{array}{c}\text { Max. res. } \\
(\AA)\end{array}$ & $\begin{array}{c}\text { Compl. } \\
(\boldsymbol{(})\end{array}$ & Red. & $\begin{array}{c}\text { Mean } \\
\boldsymbol{I}\end{array}$ & $\begin{array}{c}\text { Mean } \\
\boldsymbol{I} / \boldsymbol{\sigma}\end{array}$ & $\begin{array}{c}\boldsymbol{R}_{\text {int }} \\
(\boldsymbol{\%})\end{array}$ & $\begin{array}{c}\boldsymbol{R}_{\boldsymbol{\sigma}} \\
(\boldsymbol{\%})\end{array}$ \\
\hline $\mathbf{P}$ & 1.10 & 66.8 & 5.04 & 36.2 & 29.21 & 4.63 & 2.62 \\
$\mathbf{Q}$ & 1.10 & 65.2 & 5.02 & 35.2 & 25.74 & 5.05 & 2.93 \\
$\mathbf{R}$ & 1.10 & 66.9 & 9.38 & 37.0 & 36.46 & 4.95 & 2.13 \\
\hline
\end{tabular}

Table 3.3: Comparison of the data indicators after integrtion of $\beta$-CD form II in the three possible settings.

\begin{tabular}{c|ccc}
\hline Setting & Mean $\left|\boldsymbol{E}^{\mathbf{2}}-\mathbf{1}\right|$ & FOM & $\boldsymbol{R}_{\text {sym }}(\boldsymbol{\%})$ \\
\hline $\mathbf{P}$ & 0.772 & 0.002 & 3.7 \\
$\mathbf{Q}$ & 0.769 & 0.013 & 5.5 \\
$\mathbf{R}$ & 0.761 & 0.000 & 2.8 \\
\hline
\end{tabular}

Table 3.4: XPREP statistics for unit-cell transformations to setting $\mathbf{R}$ of the integrated data of $\beta$-CD form II.

Eq. 3.3 is a unity matrix, as the unit cell does not need to be transformed, only the $\beta$ angle should be constrained to $90^{\circ}$.

The three settings yield very similar statistics, however, $\mathbf{R}$ seems a better choice, as it provides low $R_{\text {int }}$ and $R_{\sigma}$-even considering the higher symmetry of the space group $C 222_{1}$ — but exhibits slightly larger mean intensity and intensity-to-noise ratio. Information in Table 3.4 also indicates that setting $\mathbf{R}$ should be the preferred choice, as both $\mathbf{P}$ and $\mathbf{Q}$ transformed to $\mathbf{R}$ exhibit larger $R_{\text {sym }}$ than $\mathbf{R}$ itself. Furthermore, the mean $\left|E^{2}-1\right|$ is close to the expected value for a non-centrosymmetric space group (ideally 0.736 ), while this value tends to differ in cases of twinning. Finally, given a choice of space groups belonging to different crystal systems and in the absence of twinning, it is good crystallographic practice to refine in the highest possible symmetry space group. ${ }^{25}$

A final test was performed by refining the structure in the $\mathbf{P}$ setting, with and without a twin law. This test was not performed with the $\mathbf{Q}$ setting, as it is the unlikeliest of the three possibilities. The $R$ factor of the refinement went from 11.21 to $16.06 \%$ upon the introduction of the twin law. Moreover, the final refinement in the $\mathbf{R}$ setting, space group $C 222_{1}$, provided the best refinement, and hence the possibility of twinning in the structure of $\beta$-CD form II was discarded. 


\subsubsection{Description of the structure of $\beta$-cyclodextrin form II}

$\beta$-CD form II, a 25.5 hydrate, crystallises in an orthorhombic unit cell with $a=19.7485(6) \AA, b=24.0901(6) \AA, c=32.980(5) \AA$, and space group $C 222_{1}$. Eight symmetry-related $\beta$-CD molecules are contained in a unit cell. The asymmetric unit consists of a single $\beta$-CD molecule and a disordered network of 25.5 water molecules. All glucose units within the $\beta$-CD molecule exhibit the same chair conformation. Five out of seven O6 groups are in (-)-gauche conformation, that is, pointing outwards the cavity, which is the preferred conformation. The remaining two O6 groups exhibit disorder between the $(-)$ - and $(+)$-gauche conformation, the latter being intended to stabilize water molecules inside the cavity by H-bond formation (Fig. 3.6). ${ }^{26}$ O6_5 has relative occupancies of 60 and $40 \%$ for the $(-)$ and $(+)$ conformations, respectively, while for O6_6 both conformations are equally occupied at $50 \%$. As encountered in most CD molecules, the seven O-glycosidic atoms (O4) are fairly coplanar. The root mean square (RMS) between the $\mathrm{O} 4$ atoms and their mean plane (O4 plane) is $0.020 \AA$.

Figure 3.6: Graphic definition of the $(+)$ and (-)-gauche conformations of the $\mathrm{O} 6$ groups in $\mathrm{CD}$ molecules as Newman projections of the $\mathrm{C} 5-\mathrm{C} 6$ bond. The O6 group points towards the cavity (positive O5-C5-C6-O6 torsion angle) in (+)-gauche conformation. In (-)-gauche conformation, which is the preferred one, the $\mathrm{O} 6$ group points outwards the cavity (negative O5-C5-C6-O6 torsion angle).
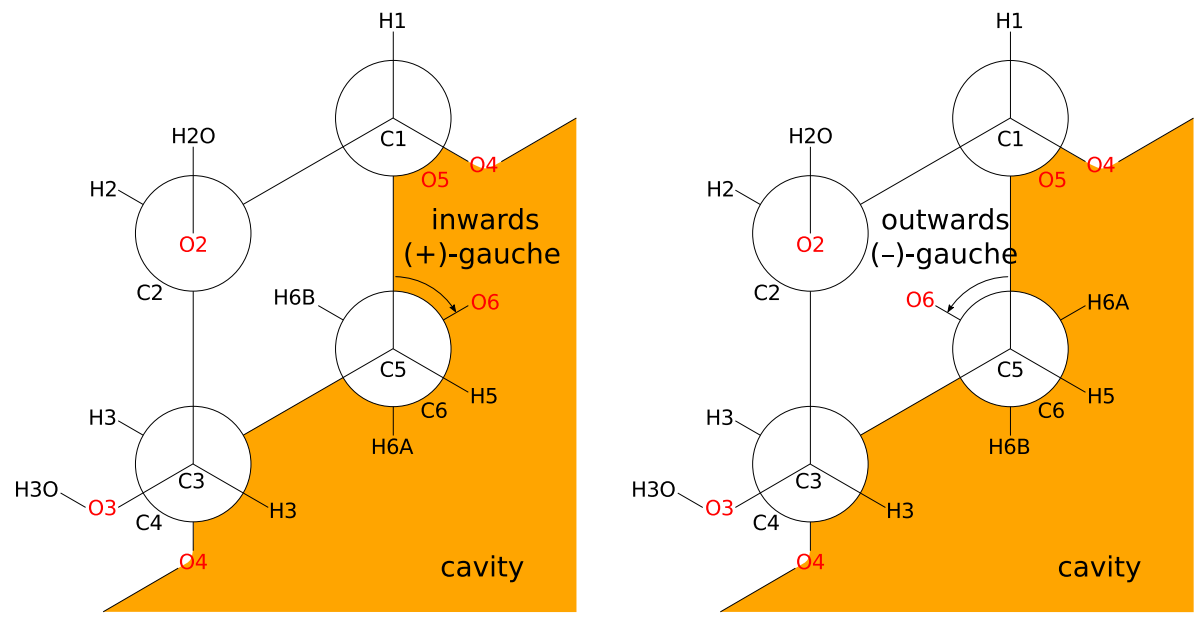

The main packing motif is a dimer of $\beta$-CD molecules (Fig. 3.7) opposed by their wider openings (O2-O3 side, usually termed as headto-head dimer). The two molecules in the dimer are related by a two-fold symmetry axis parallel to the $b$ axis of the unit cell and are linked by $\mathrm{H}$ bonds between the secondary hydroxy groups (O2 and O3). Both 
molecules are close enough (the mean plane of the atoms O2 and O3 of each molecule are separated $\sim 2.6 \AA$ ) to form a close barrel-like cavity. These dimers arrange themselves in screw channels along the $c$ axis (Fig. 3.8), thanks to the formation of $\mathrm{H}$ bonds between the $\mathrm{O} 6$ groups of two adjacent dimers, involving water molecules situated directly on top and below the dimer. The space in between dimers can be described as a wedge with an opening angle of $\sim 22^{\circ}$ (defined by the mean plane of the $\mathrm{C} 6$ atoms of each molecule). The dimers are separated $\sim 2.5 \AA$ at the closest point and $\sim 6.2 \AA$ at the furthest. From the perspective of the water molecules, the structure is better described by the combination of two different channels, one defined by the screw channel running trough the cavities of the $\beta$-CD molecules, and another defined by the space in between four adjacent screw channels (Fig. 3.9). Water molecules are likely to move along the channels, and thanks to the existence of the wedges in between $\beta$-CD dimers, they can also move from one channel to another.

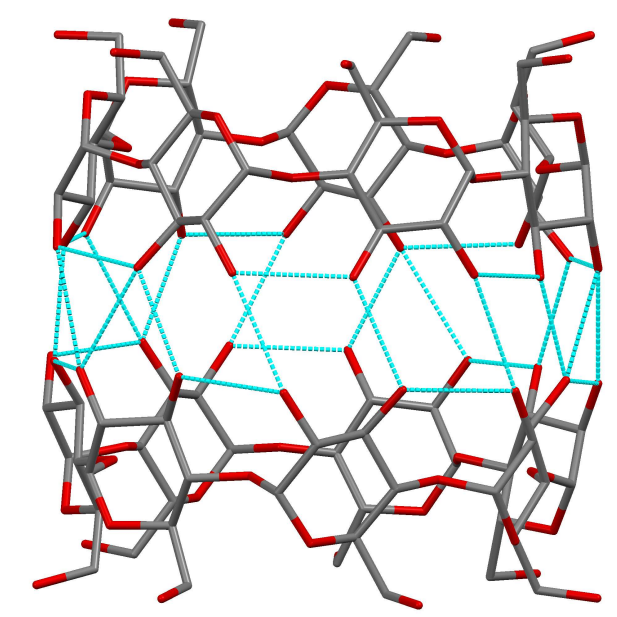

Figure 3.7: Head-to-head dimer of $\beta$-CD molecules in form II. H atoms, water molecules, and disorder have been omitted for clarity. $\mathrm{H}$ bonds between $\mathrm{O} 2$ and $\mathrm{O} 3$ groups are represented as dashed blue lines.

The packing arrangement in structures of $\beta$-CD host-guest complexes crystallising in the same space group than form II and with similar unit-cell parameters is usually referred to as a chessboard ${ }^{6}$ this particular pattern becomes apparent when the structure of the hydrate form II is projected perpendicularly to the $a$ axis (Fig. 3.10). The screw-channel packing, which is characteristic of certain $\beta$-CD structures crystallising in space group $P 22_{1}$, can also be observed in the chessboard $C 222_{1}$ 
Figure 3.8: Screwchannel packing arrangement characteristic of structures of $\beta$-CD crystallising in space group $C 222_{1}$, exemplified here for hydrate form II. Projection perpendicular to the diagonal of the $a b$ face. A channel inside $\beta$-CD cavities is represented in blue. Wedges connecting the channels inside and outside $\beta$-CD molecules are depicted in red. $\mathrm{H}$ atoms, water molecules, and disorder have been omitted for clarity.

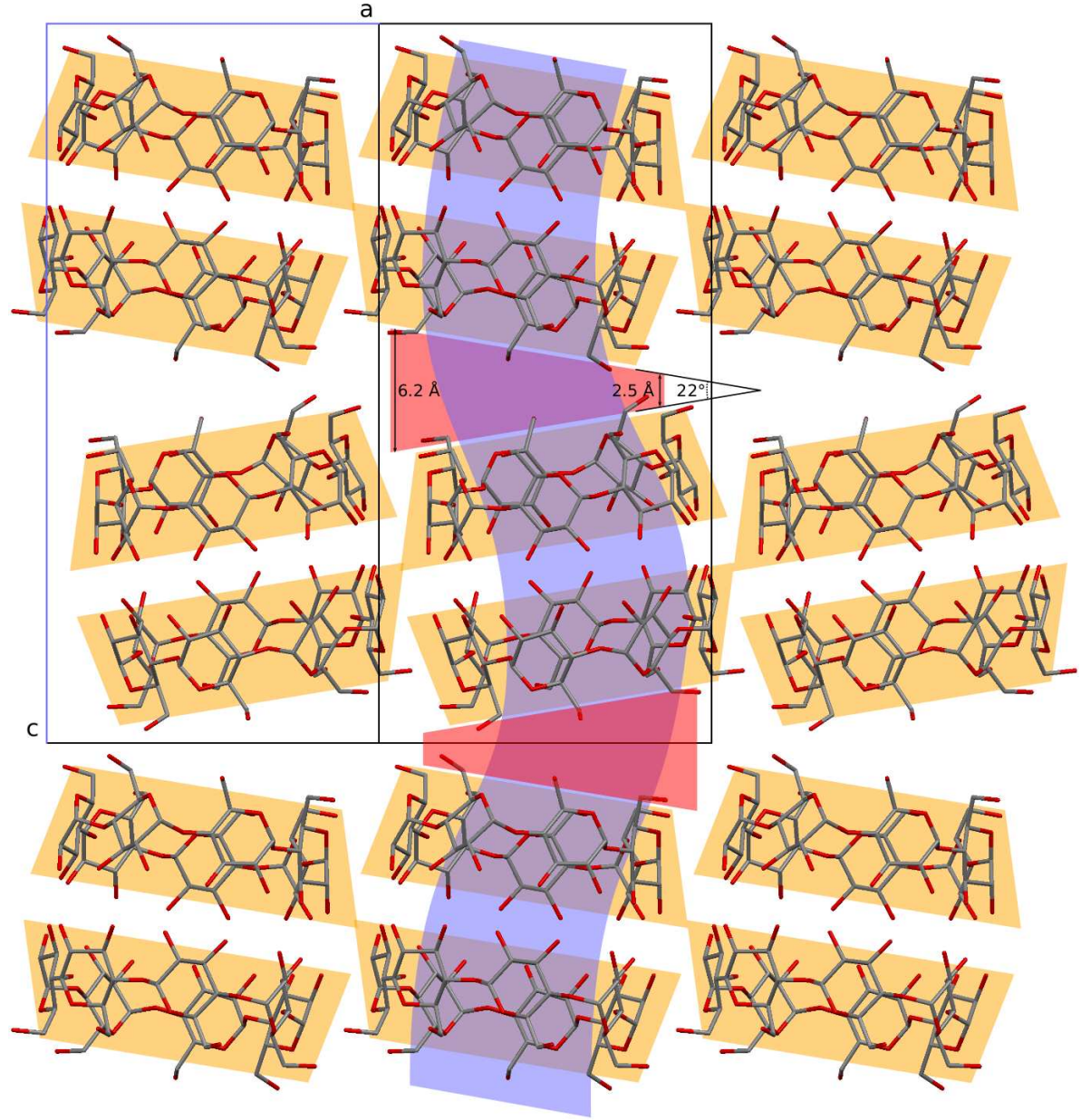

structures, in a projection perpendicular to de diagonal of the $a b$ face. These and other considerations can be found in a thorough discussion of the packing types in $\beta$-CD inclusion complexes by Saouane. ${ }^{10}$ Such discussion stemmed from the structural analysis of form $\mathrm{V}$ of $\beta$-CD-paracetamol complex, also crystallised in situ at high pressure, which is isostructural with $\beta$-CD hydrate form II reported herein. The main conclusion of the study is that the structure of $\beta$-CD.paracetamol form $\mathrm{V}$, and by extension that of $\beta$-CD hydrate form II, is slightly different than that of the rest of the $C 222_{1}$ complexes, albeit having the same unit-cell constants. In particular, some details of the packing arrangement are closer to those of the structures crystallised in space group $P 2_{1}$. In fact, the screw channels in form II hydrate are as narrow as those in the $P 2_{1}$ structures, but they are oriented like those in the $C 222_{1}$ structures. The relationship between the packing in both space groups is so 


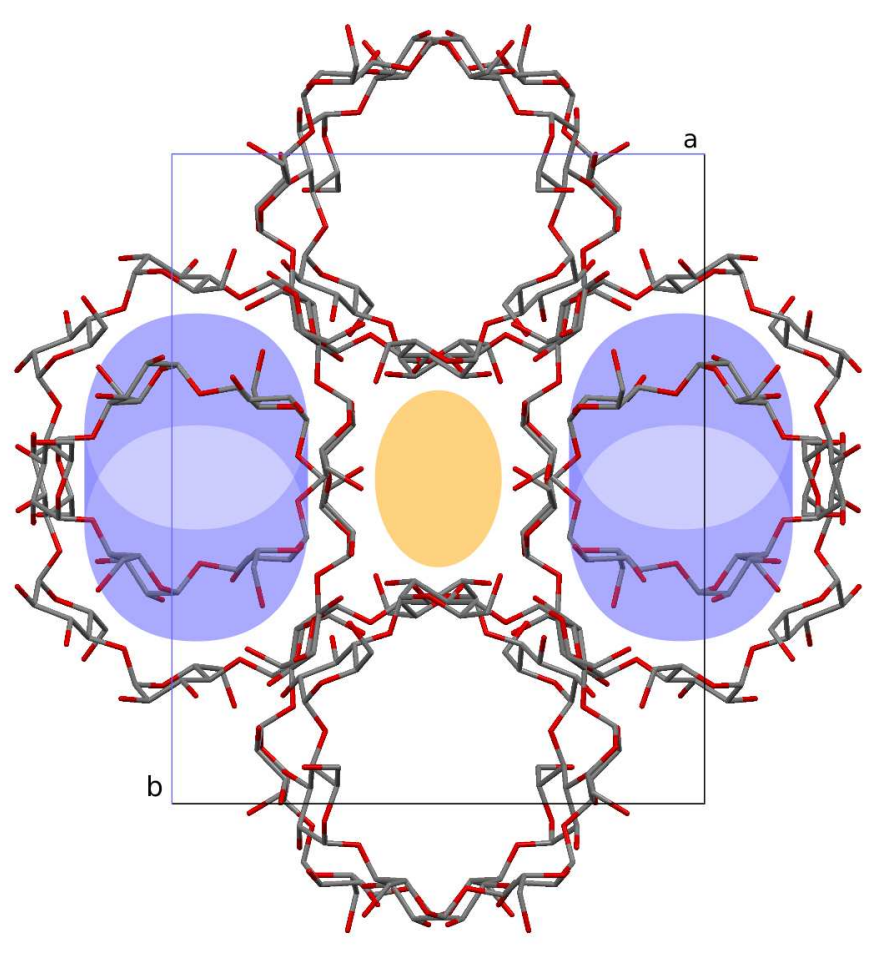

Figure 3.9: View of the channels in the structure of $\beta$-CD hydrate form II, along the axes of the channels. Cavity channels are depicted in blue, while intermolecular channels are depicted in orange.

close that it is possible to refine the structure in both $P 2_{1}$ and $C 222_{1}$, as discussed in $\S 3.3 .1$.

A possible reason for the particular packing arrangement observed in the hydrate form II and the paracetamol complex form $\mathrm{V}$ is that it allows to maximise the void volume in the structure, hence allowing for a greater water content. In order to test this hypothesis, solvent accessible volumes have been calculated using PLATON ${ }^{27}$ in all $P 2_{1}$ and $C 222_{1}$ structures, after removing water and guest molecules (Table 3.5). The calculation shows that voids per $\beta$-CD molecule in $P 2_{1}$ structures are, on average, $58.4 \AA^{3}$ smaller than in $C 222_{1}$ structures, and these are in turn $70.6 \AA^{3}$ smaller than those in the two HP structures. Such differences in void volume can account for a minimum of 2.6 and 3.2 additional water molecules, respectively. Although this is a significant difference, the change in packing alone cannot explain the super-hydration observed in the hydrate form II.

Another interesting observation is that $\beta$-CD hydrate form II and $\beta$-CD.paracetamol form $\mathrm{V}$ are isostructural and that they both show the same super-hydration effect. The Crystal Packing Similarity module 
Figure 3.10: Chessboard packing type characteristic of structures of $\beta$-CD in space group $C 222_{1}$, here exemplified with hydrate form II. Projection perpendicular to the $a$ axis. $\mathrm{H}$ atoms, water molecules, and disorder have been omitted for clarity.

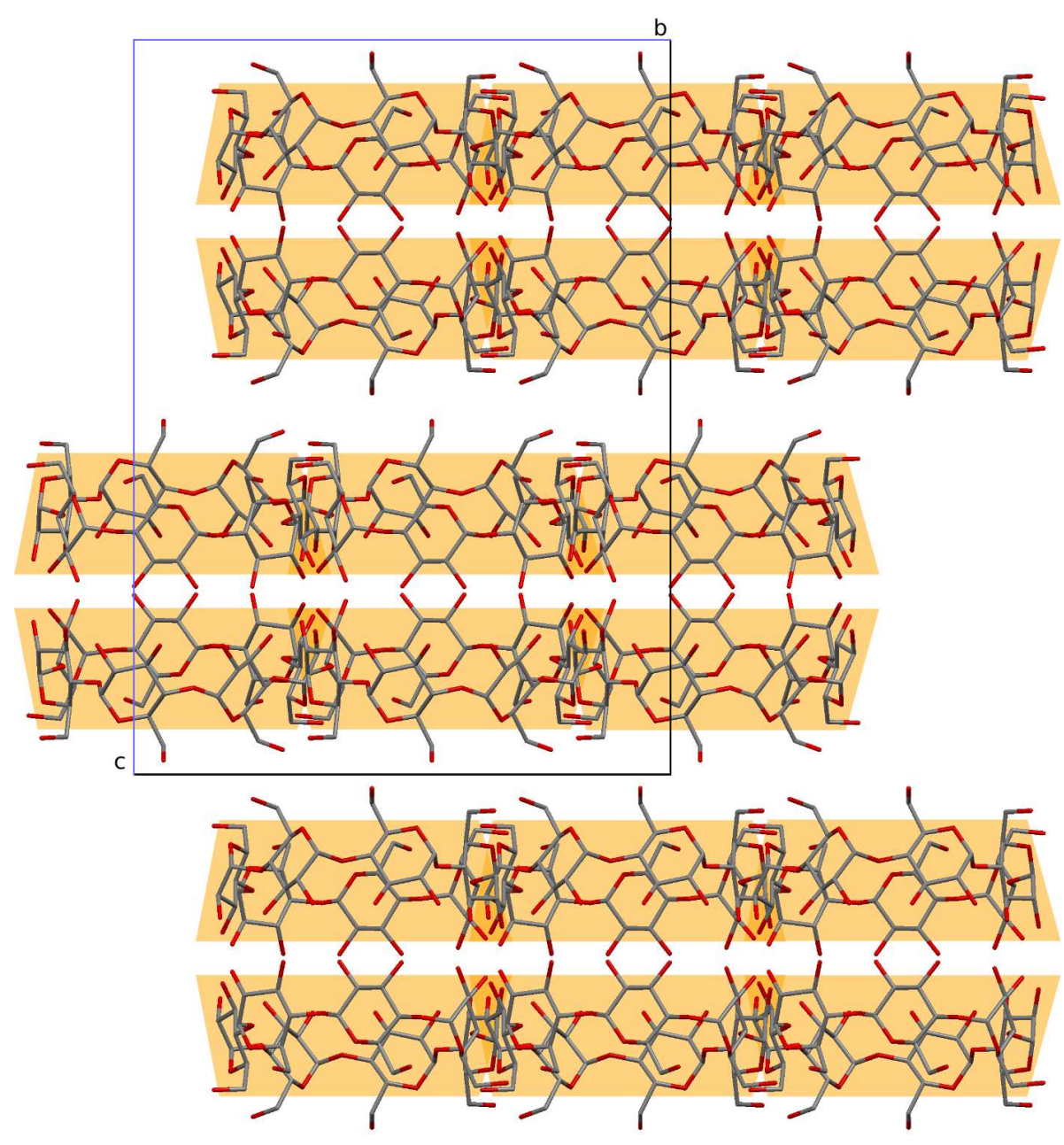

implemented in MERCURY yields an RMS difference of $0.078 \AA$ between the cores of the two structures (the core is defined as the $\beta$-CD molecule after $\mathrm{O} 2, \mathrm{O} 3, \mathrm{C} 5$, and $\mathrm{O} 6$ atoms are removed, along with water and guest molecules). In this case, the presence of the guest does not seem to alter the structure of the hydrate, which can be explained by the low occupancy of the guest, $30 \%$.

\subsubsection{Description of the structure of $\beta$-cyclodextrin form III}

Form III of $\beta$-CD, which is a 16.25 hydrate, crystallises in a triclinic unit cell with lattice parameters $a=17.8952(19) \AA, b=15.4889(12) \AA$, $c=15.5473(12) \AA, \alpha=101.061(7)^{\circ}, \beta=113.078(9)^{\circ}, \gamma=101.440(8)^{\circ}$, space group $P 1$. The are two independent $\beta$-CD molecules in the unit 
3.3. Results and discussion

\begin{tabular}{|c|c|c|c|c|}
\hline Space group & Ref. code & $V_{Z}\left(\AA^{3}\right)$ & $V_{Z, \text { void }}\left(\AA^{3}\right)$ & $V_{Z, v o i d}(\%)$ \\
\hline \multirow{3}{*}{ HP $C 222_{1}$} & Hydrate form II & 1958.6 & 816.1 & 41.7 \\
\hline & \multirow[t]{2}{*}{ Paracetamol form V } & 1961.0 & 828.5 & 42.3 \\
\hline & & $\mu \pm \sigma$ & $822.3 \pm 8.8$ & $42.0 \pm 0.4$ \\
\hline \multirow{8}{*}{$C 222_{1}$} & BOSZUF $^{28}$ & 1867.1 & 732.1 & 39.2 \\
\hline & BOTBAO $^{28}$ & 1889.9 & 748.9 & 39.6 \\
\hline & DUYVID 29 & 1879.1 & 741.0 & 39.4 \\
\hline & FALDUS ${ }^{30}$ & 1928.5 & 785.1 & 40.7 \\
\hline & LACTAL $^{31}$ & 1925.3 & 784.1 & 40.7 \\
\hline & MEGQUK $^{32}$ & 1872.4 & 732.5 & 39.1 \\
\hline & \multirow[t]{2}{*}{ WEZSIE $^{33}$} & 1869.9 & 738.1 & 39.5 \\
\hline & & $\mu \pm \sigma$ & $751.7 \pm 23.2$ & $39.8 \pm 0.7$ \\
\hline \multirow{6}{*}{$P 2_{1}$} & BEGWEQ $^{34}$ & 1819.7 & 657.6 & 36.1 \\
\hline & BOTBES $^{28}$ & 1819.7 & 670.5 & 36.8 \\
\hline & FODBIK $^{35}$ & 1880.8 & 725.9 & 38.6 \\
\hline & XEYDIQ $^{36}$ & 1871.6 & 722.8 & 38.6 \\
\hline & YOJHUC $^{37}$ & 1842.4 & 689.6 & 37.4 \\
\hline & & $\mu \pm \sigma$ & $693.3 \pm 30.6$ & $37.5 \pm 1.1$ \\
\hline
\end{tabular}

Table 3.5: Solvent accessible volumes per $\beta$-CD molecule in structures of $\beta$-CD which share lattice parameters with form II, calculated with PLATON $^{27}$

The list of structures has been taken from Saouane, ${ }^{10}$ removing structures for which not all $\mathrm{H}$ atoms have been placed. Bond distances involving $\mathrm{H}$ atoms have been elongated to neutron values using MERCURY ${ }^{38}$ prior to the calculation of $V_{\text {void }}$.

cell, whose conformation follows the same pattern as described before, with most of the O6 groups in (-)-gauche conformation. Only three O6 groups exhibit disorder. O6_13 and O6_26 both exhibit an occupancy of $70 \%$ for the $(-)$ conformation and $30 \%$ for the $(+)$. O6_22 shows relative occupancies of 60 and $40 \%$ for the $(-)$ and $(+)$ conformations, respectively. Both $\beta$-CD molecules in the unit cell are planar, with an RMS of the $\mathrm{O} 4$ atoms with respect to the $\mathrm{O} 4$ plane of 0.028 and $0.022 \AA$ for each molecule.

The same head-to-head dimer observed in other $\beta$-CD structures is repeated in this case. Even in the absence of symmetry elements relating the two $\beta$-CD molecules in the $P 1$ structure, the two components of the dimer are parallel, as evidenced by the $0.41^{\circ}$ dihedral angle between the the two O4 planes. The core dimers of both forms II and III overlap with an RMS of $0.081 \AA$. The packing arrangement in form III consists of layers of dimers perpendicular to the $a$ axis separated by layers of disordered water and displaced laterally with respect to the layers above and below (Fig. 3.11). This packing type is usually called intermediate. ${ }^{6}$

To check whether high pressure has any observable effect on the structure, a comparison between the packing of form III and 45 other structures of $\beta$-CD complexes crystallising in space group $P 1$ with sim- 
Figure 3.11: Intermediate packing arrangement of $\beta$-CD hydrate form III. Projection perpendicular to the $b$ axis. $\mathrm{H}$ atoms, water molecules, and disorder have been omitted for clarity.

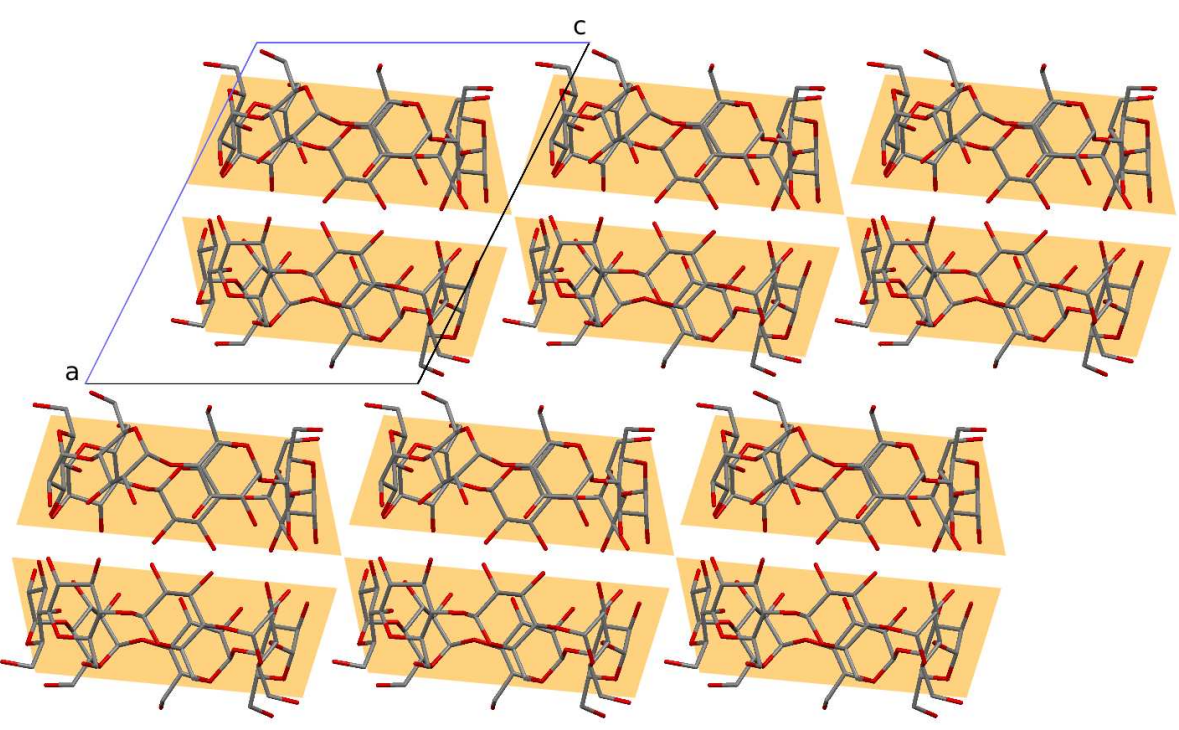

ilar unit-cell parameters has been performed with the Crystal Packing Similarity module implemented in MERCuRY, using only the core $\beta$-CD structure. Based on the RMS values obtained from the comparison (Table 3.6), form III can be considered identical to most other related $\beta$-CD structures. Only two exceptions have been found: GUMxIX, ${ }^{39}$ which exhibits the shortest of all the reduced $a$ axes (14.9878 $\AA$ against an average of $15.35 \pm 0.09 \AA)$ and also the smallest of the reduced $\alpha$ angles $\left(109.51^{\circ}\right.$ against an average of $\left.113.1 \pm 5^{\circ}\right)$; and OFOWAI, which exhibits the smallest of the reduced $\beta$ angles $\left(96.882^{\circ}\right.$ against an average of $\left.99.2 \pm 5^{\circ}\right)$ and a lack of coplanarity of the $\mathrm{O} 4$ atoms (although this was not reported as a special feature of the structure).

Similarly to what was observed in form II, the fact that this form is grown at high pressure does not seem to alter its internal structure with respect to similar structures, but still causes a dramatic rise in the hydration number.

\subsubsection{Hydration numbers of forms II and III of $\beta$-cyclodextrin}

To better understand the hydration of $\beta$-CD under high pressure, the criteria outlined in Ch. 2 have been applied. $V_{\text {excl }}$ for all three forms of $\beta$-CD are calculated in Table 3.7. There are 20 different determinations of the hydrate form I of $\beta$-CD recorded in the CSD (Appx. D). It has a 
3.3. Results and discussion

\begin{tabular}{|c|c|c|c|c|c|c|c|}
\hline Ref. code & Match $^{a}$ & RMS & $\mathbf{P X R D}^{b}$ & Ref. code & Match & RMS & PXRD \\
\hline AGAZIR 40 & 15 & 0.403 & 0.995 & FODBEG $^{35}$ & 15 & 0.420 & 0.996 \\
\hline AGAZOX 40 & 15 & 0.372 & 0.998 & GOTMOT $^{41}$ & 15 & 0.376 & 0.997 \\
\hline AGAZUD 40 & 15 & 0.505 & 0.994 & GUMXIX $^{39}$ & 12 & 1.511 & 0.979 \\
\hline AJUVAC 42 & 15 & 0.456 & 0.995 & HAHVES 43 & 15 & 0.378 & 0.997 \\
\hline ANAXAP 44 & 15 & 0.475 & 0.996 & HAKPUG 45 & 15 & 0.414 & 0.998 \\
\hline BIDMOQ $^{46}$ & 15 & 0.415 & 0.995 & HEGXUM 47 & 15 & 0.393 & 0.993 \\
\hline BOGCAB $^{48}$ & 15 & 0.396 & 0.998 & ICIYAW 49 & 15 & 0.371 & 0.998 \\
\hline BOSZEP 28 & 15 & 0.451 & 0.996 & LONGIE $^{50}$ & 15 & 0.464 & 0.993 \\
\hline BOsZIT $^{28}$ & 15 & 0.427 & 0.997 & MASBIR $^{51}$ & 15 & 0.445 & 0.996 \\
\hline BOszOz $^{28}$ & 15 & 0.382 & 0.998 & MASBOX 51 & 15 & 0.453 & 0.995 \\
\hline BURXOC 52 & 15 & 0.356 & 0.994 & NILQUV 53 & 15 & 0.479 & 0.996 \\
\hline $\mathrm{CACPOM}^{54}$ & 15 & 0.378 & 0.994 & OFOWAI ${ }^{55}$ & 9 & 1.000 & 0.993 \\
\hline CUPYOC 56 & 15 & 0.440 & 0.997 & OFOWIQ $^{57}$ & 15 & 0.378 & 0.997 \\
\hline DIFHOP 58 & 15 & 0.440 & 0.990 & TAFZIK 59 & 15 & 0.478 & 0.996 \\
\hline DOCVUM0 $4^{60}$ & 15 & 0.448 & 0.993 & TEJHAR 61 & 15 & 0.390 & 0.995 \\
\hline DOCVUM0 $6^{62}$ & 15 & 0.463 & 0.994 & UJEFEV 63 & 15 & 0.466 & 0.997 \\
\hline DOCVUM0 62 & 15 & 0.450 & 0.995 & UKAGOC 64 & 15 & 0.406 & 0.995 \\
\hline DOCVUM08 62 & 15 & 0.386 & 0.997 & VOFJIK 65 & 15 & 0.421 & 0.997 \\
\hline DOCVUM09 62 & 15 & 0.378 & 0.998 & VOQDOU 60 & 15 & 0.395 & 0.994 \\
\hline DOCVUM $10^{62}$ & 15 & 0.384 & 0.998 & VOQDUA 66 & 15 & 0.457 & 0.990 \\
\hline DOCVUM $11^{62}$ & 15 & 0.399 & 0.998 & WISREV $^{17}$ & 15 & 0.445 & 0.994 \\
\hline EYAQAX 67 & 15 & 0.486 & 0.996 & WISRIZ $^{17}$ & 15 & 0.391 & 0.994 \\
\hline EYAQEB ${ }^{67}$ & 15 & 0.436 & 0.997 & & & & \\
\hline
\end{tabular}

$a$ Out of 15 molecules.

${ }^{b}$ Degree of similarity between the PXRD patterns of both forms compared.

hydration number of 12 , which is representative of the amount of water that the structure contains if no dehydration occurs. ${ }^{7}$

Fig. 3.12 shows the $P V$ diagram for $\beta$-CD hydrates (see $\S 2.3 .3$ for a general description of the diagram). $V_{\text {excl }}$ for the two super-hydrates obtained at high pressure, forms II and III, are considerably larger than that of form I. This is an unexpected behaviour, as molecular volumes in structures obtained in situ at high pressure tend to be either similar or smaller than their AP counterparts (Ch. 2). Furthermore, the super-hydration could be explained as a way to increase the density of the crystal, but this does not seem to be the case here.

The hydration numbers of forms II and III are underestimated from the refinement, because part of the electron density is too smeared to be modelled, which leads to larger $V_{\text {excl }}$. Volume considerations have been

\begin{tabular}{lccc}
\hline Form & I & II & III \\
\hline $\boldsymbol{P}(\mathbf{G P a})$ & 0.0001 & 0.9 & 0.72 \\
$\boldsymbol{V}_{\text {cell }}\left(\AA^{\mathbf{3}}\right)$ & 3072.509 & 15688 & 3708 \\
$\boldsymbol{Z}$ & 2 & 8 & 2 \\
$\boldsymbol{V}_{\boldsymbol{Z}}$ & 1536.254 & 1961 & 1854 \\
$\boldsymbol{N}$ & 12 & 25.5 & 16.25 \\
$\boldsymbol{V}_{\boldsymbol{H}, \boldsymbol{w a t}}\left(\AA^{\mathbf{3}}\right)$ & 268.44 & 519.2 & 336.3 \\
$\boldsymbol{V}_{\text {excl }}\left(\AA^{\mathbf{3}}\right)$ & 1267.82 & 1442 & 1518 \\
\hline
\end{tabular}

Table 3.7: Molecular volume of $\beta$-CD excluding water in forms I, II and III. 
Figure 3.12: $P V$ diagram of $\beta$-CD hydrates. Empty icons represent $V_{Z}$ (higher) and $V_{\text {excl }}$ (lower). Solid icons correspond to $V_{\text {excl }}$ calculated with the minimum estimation of $N$. Solids icons with orange edge correspond to $V_{\text {excl }}$ calculated with the maximum estimation of $N$. All the points corresponding to the same form are linked by a vertical dotted line, thinner for the estimated volumes. A red dashed line indicating $V_{\text {excl }}$ of form I at ambient pressure has been included as a reference.

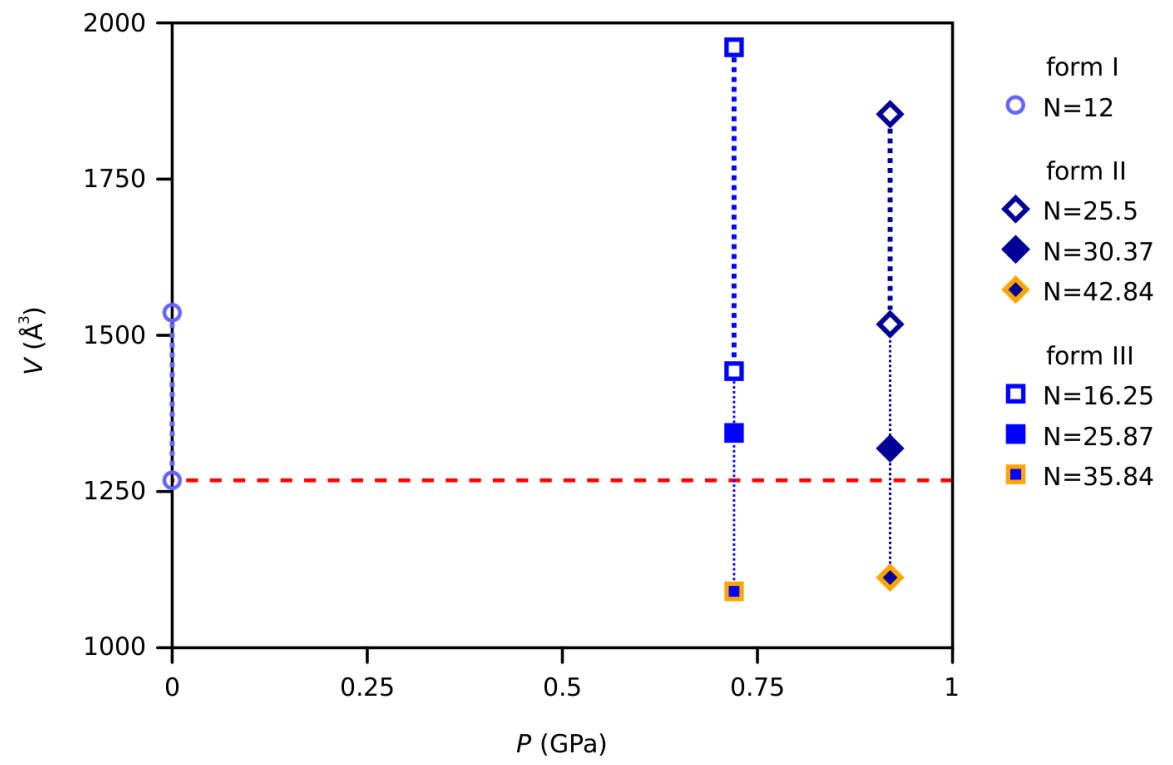

used to provide a better estimation of the hydration numbers. After removing all the water content from both structures and normalising the bonds involving $\mathrm{H}$ atoms to neutron distances, the solvent accessible volumes $\left(V_{\text {void }}\right)$ of the structures have been calculated with PLATON. ${ }^{27}$ $V_{\text {void }}$ is defined as the space inside a surface drawn $1.2 \AA$ away from the van der Waals surface of the CD molecules. The hydration number can be determined from the molecular $V_{\text {void }}\left(V_{\text {void,Z }}\right)$ applying the following equation, assuming that all the available space is filled with water molecules:

$$
N=\frac{V_{v o i d, Z}}{V_{w a t}}
$$

Where $V_{\text {wat }}$ is the volume of a single water molecule. There are indications that the behaviour of the water molecules inside the cavity of the $\beta$-CD molecule is different than those of the molecules in the intermolecular space. ${ }^{7}$ Together with the observation that $\beta$-CD molecules are rigid, it is sensible to consider that water molecules inside the cavity might not compress as much as those outside. Hence, minimum and maximum possible $V_{\text {wat }}$ have been considered to obtain a range of possible hydration numbers. The maximum $V_{w a t}$, leading to the minimum $N$, is $28.67 \AA^{3}$, which is the average volume of a water molecule in the structure of form I ( $V_{\text {void }, Z}=344.05 \AA^{3}$, occupied by 12 water molecules), assuming that there is no compression of the lattice water. This volume 
3.3. Results and discussion

\begin{tabular}{l|cccc}
\hline Form & \multicolumn{3}{c}{ II } & \multicolumn{2}{c}{ III } \\
\hline $\boldsymbol{P}(\mathbf{G P a})$ & \multicolumn{3}{|c}{0.92} & \multicolumn{2}{c}{0.72} \\
$\boldsymbol{V}_{\text {void }, \boldsymbol{Z}}\left(\AA^{\mathbf{3}}\right)$ & \multicolumn{2}{|c}{870.85} & $\boldsymbol{N}$ & $\boldsymbol{V}_{\text {excl }}\left(\AA^{\mathbf{3}}\right)$ \\
\hline $\boldsymbol{V}_{\boldsymbol{w a t}}\left(\AA^{\mathbf{3}}\right)$ & $\boldsymbol{N}$ & $\boldsymbol{V}_{\text {excl }}\left(\AA^{\mathbf{3}}\right)$ & 25.87 & 1319 \\
\hline $28.67^{a}$ & 30.37 & 1344 & 35.84 & 1112 \\
20.33 and $20.70^{a}$ & 42.84 & 1090 & \\
\hline
\end{tabular}

Table 3.8: Hydration numbers $(N)$ of $\beta$-CD hydrates estimated from volume considerations.

${ }^{a}$ Average volume of a water molecule in the structure of form I.

${ }^{b}$ Determined from the compressibility curve of lattice water at 0.92 and $0.72 \mathrm{GPa}$, respectively.

is considerably larger than the average $22.37 \AA^{3}$ estimated in $\S 2.3 .1$, but is deemed more appropriate considering the particularities of $\beta$-CD. The minimum $V_{\text {wat }}$, leading to the maximum $N$ and determined from the compressibility curve of lattice water, is $20.33 \AA^{3}$ at $0.92 \mathrm{GPa}$ for form II and $20.70 \AA^{3}$ at $0.72 \mathrm{GPa}$ for form III. Estimated hydration numbers, and the values of $V_{\text {excl }}$ they lead to, can be found in Table 3.8.

The estimated $V_{\text {excl }}$ are also included in Fig. 3.12. The results indicate that the hydration numbers refined from X-ray diffraction data are underestimated. This can also be checked by dividing $V_{\text {void, } Z}$ by $N$ as determined during the refinement (Table 3.7), which gives $V_{\text {wat }}$ of 34.15 and $45.64 \AA^{3}$ for forms II and III, respectively, considerably larger than the maximum $V_{w a t}$ determined above. Nevertheless, considering the lower end of the range of estimated values for the hydration number yields $V_{\text {excl }}$ larger than that of form I. For the $\beta$-CD molecule to exhibit lower $V_{\text {excl }}$ in forms II and III than in form I it will be necessary to have hydration numbers larger than 32.30 and 26.60, respectively. Those numbers should still be larger to account for the fact that $V_{\text {excl }}$ of form I will be smaller at higher pressures. This last contribution cannot be estimated, as the compressibility curve of $\beta$-CD form I has not been measured. Based in similar experiences (§5.2.1), such an experiment is not straightforward, given that when using solvents such as water, methanol, or ethanol, form I dissolves with increasing pressure. The experiment would require finding a non penetrating, non interacting PTM and ensuring that the hydration number remains constant during compression.

With this information, two different scenarios can be suggested. The first option is that $\beta$-CD forms II and III exhibit in fact smaller $V_{\text {excl }}$ than 
form I. If this were the case, the hydration numbers would be severely underestimated in this study. It would be necessary to collect better data or provide better estimations for the hydration numbers and compare the resulting $V_{\text {excl }}$ with those in the compressibility curve of form I. This scenario seems unlikely given the observations made in §3.3.2. In the first place, form II shows the same unit-cell parameters than other structures of $\beta$-CD complexes at ambient pressure. This could indicate that the crystal does not compress, but rather acts as a storage of water, for the solution to increase its density as much as possible. The solution surrounding the crystal at $0.9 \mathrm{GPa}$ can increase its density by having most of its water H-bonded to $\beta$-CD molecules, hence decreasing their mobility. The excess water is then passed on to the crystal. Second, the analysis of void volumes suggests that either all structures of $\beta$-CD reported up to date have been refined with underestimated hydration numbers, or else the volume occupied by water molecules in such structures is larger than the one observed in crystals of hydrates of other organic substances (Table 3.9). The latter, more likely, might indicate a large availability of space which can be filled with extra water molecules to achieve the super-hydrated state without the need of compressing the structure of the crystal; or, if water molecules are less compressible than expected in $\beta$-CD crystals, that the hydration number, at least in form II, tends towards the lower end of the range given above.

Table 3.9: Average volume of lattice water molecules in $\beta$-CD structures, calculated from $V_{\text {void }}$ and $N$ determined from diffraction data (Table $3.5^{a}$ )

\begin{tabular}{|c|c|c|c|c|}
\hline $\begin{array}{l}\text { Space } \\
\text { group }\end{array}$ & Ref. code & $V_{Z, \text { void }}\left(\AA^{3}\right)$ & $N$ & $V_{w a t}\left(\AA^{3}\right)$ \\
\hline \multirow{4}{*}{$C 222_{1}$} & BOSZUF $^{28}$ & 426.9 & 13.5 & 31.6 \\
\hline & вотвАО $^{28}$ & 324.6 & 10.5 & 30.9 \\
\hline & DUYVID $^{29}$ & 292.0 & 10.1 & 28.9 \\
\hline & WEZSIE $^{33}$ & 364.5 & 10.0 & 36.4 \\
\hline \multirow{4}{*}{ HP $P 2_{1}$} & BEGWEQ $^{34}$ & 347.0 & 14.0 & 24.8 \\
\hline & BOTBES $^{28}$ & 289.4 & 11.3 & 25.7 \\
\hline & FODBIK $^{35}$ & 347.2 & 10.8 & 32.3 \\
\hline & YOJHUC $^{37}$ & 281.5 & 11.8 & 24.0 \\
\hline
\end{tabular}

a Differently from Table $3.5, V_{Z, \text { void }}$ are calculated here removing only water molecules, but leaving the guests. Structures FALDUS and MEGQUK have been removed because the content of their cavities has not been determined experimentally. Structures LACTAL and XEYDIQ have been removed because both yielded very large volumes for water molecules, probably due to a small guest which does not fill completely the cavity. In such situation the remaining void volume of the cavity is distributed over all the water molecules. 
In the second option, $V_{\text {excl }}$ for forms II and III are larger than that of form I. If this were the case, the density and the concentration of the solution surrounding crystals of forms II or III inside the sample chamber should be considerably larger than that of a saturated aqueous solution of $\beta-C D$ at ambient pressure. This is because the application of pressure leads invariably to a decrease in the volume of the system. If the HP crystals are not denser than their AP counterparts, the solution around them must be denser. An experiment to test this scenario has been designed but not carried out yet. The basic idea is to determine the hydration number of form II by measuring the mass of $\beta$-CD and water in the crystal. These parameters cannot be measured directly, but can be derived from the size of the crystals and the amount of solution and its density, which can be determined spectroscopically. The main problem of this method is the determination of the volume of the sample chamber, as its depth cannot be measured accurately. This can be overcome if the total mass of $\beta-\mathrm{CD}$ and water inside the sample chamber are known.

\subsection{Conclusions and outlook}

Two new hydrates of $\beta$-CD, with 25.5 and 16.25 water molecules per CD molecule, have been crystallised in situ at high pressure and their crystal structures determined and described. The results provide further evidence of the ability of high-pressure techniques to allow access to crystalline forms which cannot be observed by other means. The two structures provide for the first time evidence of super-hydration in a purely organic compound. Furthermore, the use of pressure has exposed the complexity of an otherwise well-characterised system.

Crystals of $\beta$-CD form I, a dodecahydrate, are known to undergo a reversible dehydration process, controlled by the humidity of their environment. The movement of water molecules through the crystal structure, hindered by the tightness of the herringbone packing, is made possible by a concerted conformational change of flip-flop $\mathrm{H}$ bonds across the crystal. Forms II and III, 25.5 and 16.25 hydrates, respectively, can be crystallised at 0.92 and $0.72 \mathrm{GPa}$ from $\beta$-CD aqueous saturated solu- 
tion. Both forms were obtained following an established crystallisation protocol, albeit form III crystallised serendipitously and more experiments are needed to make the crystallisation of this form reproducible, in contrast with form II, which can be obtained routinely.

Both forms can be considered super-hydrates and exhibit a rather different behaviour than form I. Unfortunately, single-crystal X-ray diffraction studies have not allowed to accurately determine the hydration numbers of these new forms. Notwithstanding this, the hydration numbers inferred from structure refinement allow to at least make a number of valid speculations. On the one hand, if the hydration numbers were indeed to be correct, both forms would be considerably less dense than the AP form I, which contravenes the usual behaviour observed for crystals obtained under high pressure, and would force the solution surrounding the crystals at high pressure to be extremely dense. On the other hand, if the crystals were to exhibit the expected density at their formation pressures, the hydration numbers should be considerably higher, emphasizing the super-hydration features of $\beta$-CD molecules. The collection of diffraction data on more specimens at higher resolutions, possibly combined with the use of low temperature (e.g. with a close-cycle cryostat), which should enable better modelling of the disorder, or the measurement of the densities of the solution inside the DAC along the crystallisation process, by spectroscopic techniques, might allow to determine more accurate hydration numbers and shed light on the formation and behaviour of both forms II and III, which in turn will provide a better understanding on how the hydration process occurs. 


\section{References}

[1] J. A. Hamilton, L. K. Steinrauf and R. L. Van Etten, Acta Cryst., 1968, B24, 1560-1562.

[2] K. Lindner and W. Saenger, Angew. Chem. Int. Ed., 1978, 17, 694695.

[3] K. Lindner and W. Saenger, Carbohydr. Res., 1982, 99, 103-115.

[4] W. Saenger, M. Noltemeyer, P. C. Manor, B. Hingerty and B. Klar, Bioorg. Chem., 1976, 5, 187-195.

[5] W. Saenger, Angew. Chem. Int. Ed., 1980, 19, 344-362.

[6] D. Mentzafos, I. M. Mavridis, G. Le Bas and G. Tsoucaris, Acta Cryst., 1991, B47, 746-757.

[7] T. Steiner and G. Koellner, J. Am. Chem. Soc., 1994, 116, 51225128.

[8] C. Betzel, W. Saenger, B. E. Hingerty and G. M. Brown, J. Am. Chem. Soc., 1984, 106, 7545-7557.

[9] W. Saenger and T. Steiner, Acta Cryst., 1998, A54, 798-805.

[10] S. Saouane, PhD thesis, Georg-August-Universität Göttingen, 2015.

[11] Y. Lee, J. A. Hriljac, T. Vogt, J. B. Parise and G. Artioli, J. Am. Chem. Soc., 2001, 123, 12732-12733.

[12] D. Seoung, Y. Lee, C.-C. Kao and T. Vogt, Chem. Eur. J., 2013, 19, 10876-10883.

[13] A. J. Graham, D. R. Allan, A. Muszkiewicz, C. A. Morrison and S. A. Moggach, Angew. Chem. Int. Ed., 2011, 50, 11138-11141.

[14] C. Paulmann, MARTOOLSCP, 2007.

[15] Bruker-AXS, APEX2, Bruker-AXS, Madison, Wisconsin, USA, 2007.

[16] CRYSALIS PRO v171.36.28, Rigaku Oxford Diffraction, Tokyo, Japan, 2012.

[17] S. Makedonopoulou and I. M. Mavridis, Acta Cryst., 2000, B56, 322-331.

[18] G. M. Sheldrick, Acta Cryst., 2015, C71, 3-8.

[19] C. B. Hübschle, G. M. Sheldrick and B. Dittrich, J. Appl. Cryst., 2011, 44, 1281-1284. 
[20] GRADE, http://grade.globalphasing.org.

[21] G. M. Sheldrick, SADABS v2008-1, Bruker-AXS, Madison, Wisconsin, USA, 2008.

[22] W. C. Hamilton, Acta Cryst., 1965, 18, 502-510.

[23] A. T. Brünger, Nature, 1992, 355, 472-475.

[24] G. M. Sheldrick, XPREP v2012-1, Bruker-AXS, Madison, Wisconsin, USA, 2012.

[25] D. Watkin, Acta Cryst., 1994, A50, 411-437.

[26] K. K. Chacko and W. Saenger, J. Am. Chem. Soc., 1981, 103, $1708-1715$.

[27] A. L. Spek, Acta Cryst., 2009, D65, 148-155.

[28] Y. L. Zhao, D. Benítez, I. Yoon and J. F. Stoddart, Chem. Asian J., 2009, 4, 446-456.

[29] G. D. Enright, K. A. Udachin and J. A. Ripmeester, CrystEngComm, 2010, 12, 1450-1453.

[30] L. Damodharan, V. Pattabhi and K. Nagarajan, Mol. Cryst. Liq. Crys., 2004, 423, 17-35.

[31] Y. Liu, Y. L. Zhao, H. Y. Zhang, X. Y. Li, P. Liang, X. Z. Zhang and J. J. Xu, Macromolecules, 2004, 37, 6362-6369.

[32] M. M. Bobek, G. Giester, H. Kählig and U. H. Brinker, Tetrahedron Lett., 2000, 41, 5663-5667.

[33] P. M. Dean, Aust. J. Chem., 2007, 60, 133-138.

[34] M. R. Caira, E. De Vries, L. R. Nassimbeni and V. W. Jacewicz, J. Incl. Phenom. Macrocycl. Chem., 2003, 46, 37-42.

[35] Y. Liu, Y.-L. Zhao, Y. Chen and D.-S. Guo, Org. Biomol. Chem., 2005, 3, 584-591.

[36] M. Ceborska, M. Asztemborska and J. Lipkowski, Chem. Phys. Lett., 2012, 553, 64-67.

[37] T. Cappadona, L. Daniels and T. Siddiquee, Appl. Sci., 2012, 2, 773-779.

[38] C. F. Macrae, I. J. Bruno, J. A. Chisholm, P. R. Edgington, P. McCabe, E. Pidcock, L. Rodriguez-Monge, R. Taylor, J. van de Streek and P. A. Wood, J. Appl. Cryst., 2008, 41, 466-470. 
[39] N. V. Sule, A. Ugrinov, S. Mallik and D. K. Srivastava, Biochim. Biophys. Acta, 2015, 1850, 141-149.

[40] J. M. Alexander, J. L. Clark, T. J. Brett and J. J. Stezowski, Proc. Nat. Acad. Sci. USA, 2002, 99, 5115-5120.

[41] J. A. Fernandes, A. I. Ramos, P. R. Claro, F. A. Almeida Paz and S. S. Braga, CrystEngComm, 2015, 17, 937-946.

[42] M. R. Caira, E. J. C. de Vries and L. R. Nassimbeni, Chem. Commun., 2003, 2058-2059.

[43] X.-J. Shen, H.-L. Chen, F. Yu, Y.-C. Zhang, X.-H. Yang and Y.-Z. Li, Tetrahedron Lett., 2004, 45, 6813-6817.

[44] M. L. Singleton, J. H. Reibenspies and M. Y. Darensbourg, J. Am. Chem. Soc., 2010, 132, 8870-8871.

[45] E. Wang, G. Chen and H. Liu, Chin. J. Chem., 2009, 27, 2097-2101.

[46] M. B. Hursthouse, C. Z. Smith, M. Thornton-Pett and J. H. P. Utley, J. Chem. Soc., Chem. Commun., 1982, 881-882.

[47] A. Rontoyianni, I. M. Mavridis, E. Hadjoudis and A. J. M. Duisenberg, Carbohydr. Res., 1994, 252, 19-32.

[48] J. A. Hamilton and M. N. Sabesan, Acta Cryst., 1982, B38, 30633069 .

[49] S. M. Bruno, J. A. Fernandes, J. Marques, S. C. Neto, P. J. RibeiroClaro, M. Pillinger, F. A. Almeida Paz, M. P. M. Marques, S. S. Braga and I. S. Gonçalves, Eur. J. Inorg. Chem., 2011, 4955-4963.

[50] S. Makedonopoulou, A. Tulinsky and I. M. Mavridis, Supramol. Chem., 1999, 11, 73-81.

[51] T. J. Brett, J. M. Alexander and J. J. Stezowski, J. Chem. Soc., Perkin Trans. 2, 2000, 1105-1111.

[52] A. Paulidou, D. Maffeo, K. Yannakopoulou and I. M. Mavridis, CrystEngComm, 2010, 12, 517-525.

[53] Z. Fan, C.-H. Diao, M.-J. Guo, R.-J. Du, Y.-F. Song, Z.-L. Jing and M. Yu, Carbohydr. Res., 2007, 342, 2500-2503.

[54] S. Makedonopoulou and I. M. Mavridis, Carbohydr. Res., 2001, 335, 213-220.

[55] Z.-X. Yang, Y. Chen and Y. Liu, Carbohydr. Res., 2008, 343, 24392442. 
[56] N. R. Lien and J. R. Telford, Carbohydr. Res., 2009, 344, 26062608.

[57] T. Aree and N. Chaichit, Carbohydr. Res., 2008, 343, 2285-2291.

[58] F. Nishioka, I. Nakanishi, T. Fujiwara and K.-I. Tomita, J. Inclusion Phenom., 1984, 2, 701-714.

[59] T. Bojinova, H. Gornitzka, N. Lauth-de Viguerie and I. Rico-Lattes, Carbohydr. Res., 2003, 338, 781-785.

[60] J. L. Clark and J. J. Stezowski, J. Am. Chem. Soc., 2001, 123, 9880-9888.

[61] A. Rontoyianni and I. M. Mavridis, Acta Cryst., 1996, C52, 22772281.

[62] J. L. Clark, J. Peinado, J. J. Stezowski, R. L. Vold, Y. Huang and G. L. Hoatson, J. Phys. Chem. B, 2006, 110, 26375-26387.

[63] E. J. Wang, G. Y. Chen and C. R. Han, Acta Chim. Sinica, 2009, 67, 2527-2530.

[64] Y. Liu, Y.-L. Zhao, H.-Y. Zhang and H.-B. Song, Angew. Chem. Int. Ed., 2003, 42, 3260-3263.

[65] Y. Zhang, S. Yu and F. Bao, Carbohydr. Res., 2008, 343, 25042508 .

[66] J. L. Clark, B. R. Booth and J. J. Stezowski, J. Am. Chem. Soc., 2001, 123, 9889-9895.

[67] E. Wang, G. Chen and C. Han, Chin. J. Chem., 2011, 29, 617-622. 
CHAPTER 4

\section{$\boldsymbol{\beta}$-Cyclodextrin dimethylformamide 12.5 hydrate inclusion complex}

The contents of this chapter are published in R. Granero-García amd F. P. A. Fabbiani, Acta. Cryst., 2014, B70, 586-594. Only my personal contribution to the results presented in this paper are included here.

\subsection{Introduction}

Already in 1991 Mentzafos et al. showed that certain packing types of $\beta$-CD are associated with certain space groups and unit-cell parameters and presented a classification of the dimeric $\beta$-CD complexes according to this analysis ${ }^{1}$. In their seminal 1998 review article, Saenger and Steiner summarized the most likely packing types adopted by $\beta$-CD complexes depending on the size of the macrocycle and the nature of the guest. $^{2}$ When water or small guests are included, the preferred mode of packing of $\beta$-CD molecules follows a herringbone motif, whilst in the presence of bigger guests a channel arrangement is favoured. In the 
particular case of space group $P 2_{1}$, in which the here reported $\beta$-CD dimethylformamide (DMF) complex crystallizes, two types of packing are predominant: herringbone, associated with the unit-cell parameters $a \sim 15 \AA, b \sim 10 \AA, c \sim 21 \AA, \beta \sim 110^{\circ}$; and dimeric layers, associated with $a \sim 15 \AA, b \sim 32 \AA, c \sim 15 \AA, \beta \sim 102^{\circ}$. Exceptions exist where these packing types appear and the resulting unit-cell parameters are slightly different from the ones just outlined. The structure of $\beta$-CD-DMF belongs to one of these exceptions and shows the largest deviations observed thus far. This chapter presents a thorough analysis of the packing motifs of structurally related $\beta$-CD complexes using the structural similarity concept incorporated in the XPAC method. ${ }^{3}$

\subsection{Experimental}

\subsubsection{Crystal growth}

Crystals of the $\beta$-CD-DMF complex were grown by slow evaporation from an aqueous saturated solution of $\beta$-CD (Sigma-Aldrich, used as received) to which a few drops of DMF (Sigma-Aldrich, used as received) were added. The crystals grew in the shape of long, thin, transparent laths.

\subsubsection{X-ray diffraction analysis}

X-ray diffraction data were collected on one of these crystals at $120 \mathrm{~K}$ on a Bruker ApexII CCD diffractometer equipped with a Mo microsource $(\lambda=0.71073 \AA)$. The data collection strategy consisted of $\omega$ scans with scan steps of $0.3^{\circ}$ and exposure times of $60 \mathrm{~s}$ per frame. Data were integrated following the strategy outlined in $\S 1.1 .3$, without the high-pressure corrections, which were not needed in this case. The structure was solved using the integrated Patterson and direct methods procedure implemented in the program $\operatorname{PATSEE}^{4}$ and full-matrix least-squares refinement against $F^{2}$ was subsequently performed using SHELXL V $2013^{5}$ through the GUI SHELXLE. ${ }^{6}$

All non-H atoms were refined anisotropically, with the exception of some of the disordered atoms, namely: O60_1 in the $\beta$-CD macrocycle, 
the disordered atoms in the DMF molecule $\left(\mathrm{C} 2, \mathrm{C} 2{ }^{\prime}, \mathrm{N} 3, \mathrm{~N} 3^{\prime}, \mathrm{C} 5\right.$, and $\left.\mathrm{C} 5^{\prime}\right)$, and the $\mathrm{O}$ atoms of some of the water molecules (O9, $\mathrm{O}^{\prime}, \mathrm{O} 11$, O11', and O11"; Fig. 4.1). The geometry of all the glucose rings was restrained to be the same. $\beta$-CD $\mathrm{H}$ atoms were initially placed geometrically and constrained using the riding model; some positions were later improved by looking at difference Fourier maps. $\mathrm{H}$ atoms belonging to water molecules were located in Fourier difference maps when possible, their positions optimized according to the formation of possible $\mathrm{H}$ bonds and then refined subject to restraints. In the case of the water molecules labelled O10, O11 and O12 (with their respective disordered positions) $\mathrm{H}$ atoms could not be located. Site-occupancy factors were initially refined and subsequently fixed. An iterative refinement procedure of the highly correlated site-occupancy factors and displacement parameters using different starting models ensured refinement convergence and a reasonable model. A final $R$ factor of $5.62 \%$ was obtained. For further crystallographic data see Table 4.1.

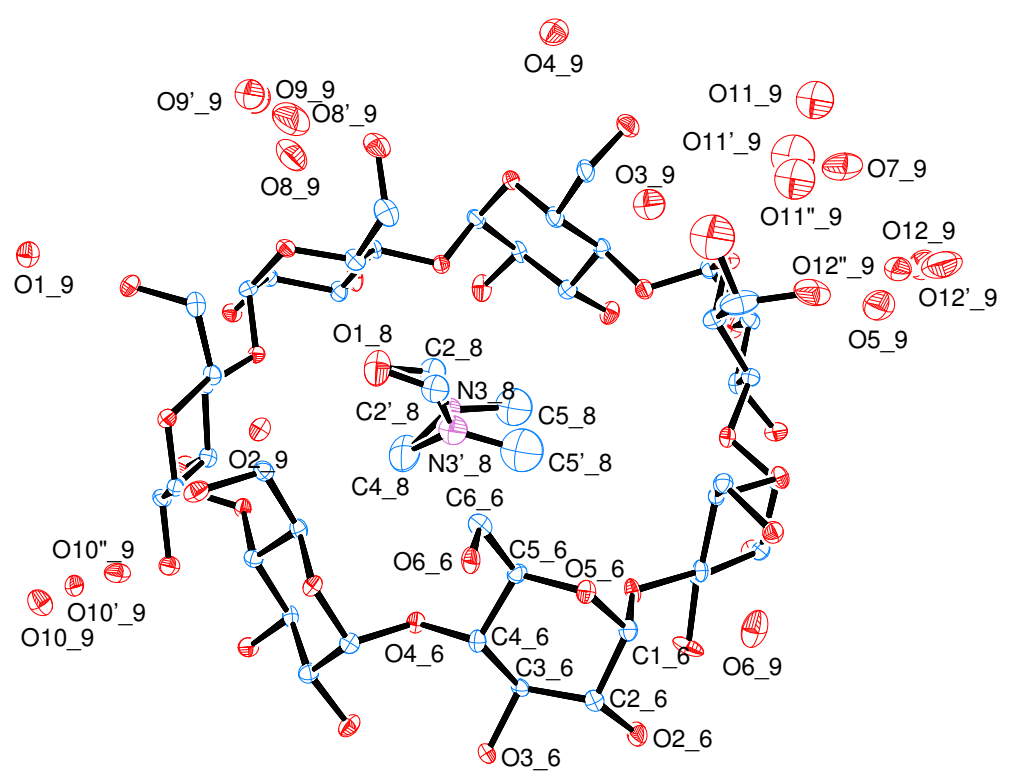

Figure 4.1: Chemical diagram of $\beta$-CD with numbering scheme. For simplicity only one of the glucose fragments is labelled.

\subsubsection{XPAC analysis}

A structure comparison among complexes of $\beta$-CD stored in the CSD (v5.34 with updates to May 2013) was carried out in an attempt to clarify the relationship between the unit-cell parameters, the crystal pack- 


\section{4. $\beta$-Cyclodextrin dimethylformamide 12.5 hydrate inclusion complex}

Table 4.1: Crystallographic data and refinement parameters of $\beta$-CDDMF $12.5 \mathrm{H}_{2} \mathrm{O}$.

\begin{tabular}{ll}
\hline Crystal data & \\
\hline Chemical formula & $\mathrm{C}_{42} \mathrm{H}_{70} \mathrm{O}_{35}-\mathrm{C}_{3} \mathrm{H}_{7} \mathrm{NO} \cdot 25.5 \mathrm{H}_{2} \mathrm{O}$ \\
Molecular mass $\left(\mathrm{g} \mathrm{mol}^{-1}\right)$ & 1433.27 \\
Temperature $(\mathrm{K})$ & $120(2)$ \\
Crystal system, space group & monoclinic, $P 2_{1}$ \\
$a, b, c(\AA)$ & $15.500(3), 9.794(2), 21.689(5)$ \\
$\beta\left({ }^{\circ}\right)$ & $90.138(3)$ \\
$V\left(\AA^{3}\right)$ & $3292.5(12)$ \\
$Z$ & 2 \\
Crystal size $(\mathrm{mm})$ & $\sim 0.2 \times 0.1 \times 0.05$ \\
\hline
\end{tabular}

\section{Data collection}

Radiation wavelength $(\AA)$

Absorption correction

$T_{\min }, T_{\max }$

Number of $[I>2 \sigma(I)]$ reflections

reflections:

- measured 38760

- independent 13449

- observed 11103

$(\sin \theta / \lambda)_{\max }\left(\AA^{-1}\right) \quad 0.626$

$R_{\text {int }}(\%) \quad 5.29$

\begin{tabular}{ll}
\hline Refinement & \\
\hline$R\left[F^{2}>2 \sigma\left(F^{2}\right)\right](\%)$ & 5.60 \\
$w R\left(F^{2}\right)(\%)$ & 13.00 \\
$S$ & 1.12 \\
Number of reflections & 13449 \\
Number of parameters & 959 \\
Number of restraints & 564 \\
$\Delta \rho_{\min }, \Delta \rho_{\max }\left(\mathrm{e} \AA^{-3}\right)$ & $-0.34,0.46$ \\
\hline
\end{tabular}

ing and the chemical composition of this type of material. The XPAC method ${ }^{3}$ was used for this purpose. This method is based on the comparison of a cluster of molecules built around a central one, for which a set of atomic positions can be freely defined by the user. Clusters for every structure to be compared are analysed in terms of all possible angles and interplanar angles between molecules in the clusters, on the basis of user-selected predefined atoms. Values are compared across all clusters and mean differences are output as a series of parameters. This methodology allows a quantitative comparison across a large number of structures to be performed in a very efficient way. Additionally, the method yields a dissimilarity index ${ }^{8}$ that can be used to quickly assess the degree of structural similarity. The method also identifies whether supramolecular constructs that are common to the compared structures exist, if any, and then determines their dimensionality (zero-, one-, twoor three-dimensional). 
Several authors presenting structures of $\beta$-CD inclusion complexes report parameters based on the mean plane for the seven glycosidic $\mathrm{O}$ atoms (O4 plane) in order to characterize molecular shape. ${ }^{9-15}$ Hence, these were the atoms also used to define molecules in the XPAC study, along with the carbon atoms linked to them (C1 and $\mathrm{C} 4$ of every glucose ring). This choice of atoms also allows to take into account slight distortions of the macrocycle. A subset of $38 \beta$-CD structures was obtained from the CSD. Only structures in the space group $P 2_{1}$, exhibiting the herringbone packing motif, with similar unit-cell parameters, and with deposited three-dimensional coordinates were chosen. A list of all the structures included in the XPAC calculation can be found in Table 4.2 (for complete information on unit-cell parameters see Appx. E). MUPNEQ01, whose structure was determined by powder diffraction, ${ }^{11}$ was found to fulfil all the prerequisites of the CSD search but was not included in the XPAC search because of the highly distorted geometry of the $\beta$-CD molecule. All XPAC calculations were performed with the program's default values.

\begin{tabular}{llll}
\hline ARUXIU $^{14}$ & DEWCOY $^{16}$ & MIFHAK $^{17}$ & UPULEX $^{18}$ \\
ARUXOA $^{14}$ & DIRVOP $^{19}$ & NUFVEQ $^{20}$ & VACZIJ $^{12}$ \\
BCDEXD03 $^{21}$ & GUXZOO $^{22}$ & NUFVIU $^{20}$ & VOJLIQ $^{23}$ \\
BCDEXD04 $^{21}$ & HAQNUK $^{24}$ & PIJGIY $^{25}$ & XEGTAF $^{26}$ \\
BCDEXD05 $^{21}$ & IZALEB $^{27}$ & PIJGOE $^{25}$ & YIYSII $^{28}$ \\
BCDEXD10 $^{9}$ & KIJSEC $^{29}$ & POBRON $^{21}$ & YIYSOO $^{28}$ \\
BOBPEN $^{30}$ & KOBRIC $^{10}$ & POBRON01 $^{31}$ & ZIGZIY $^{32}$ \\
BOBPIR $^{30}$ & KUTKOZ $^{33}$ & POVSIC $^{34}$ & -CD-DMF $^{35}$ \\
BUVSEQ $^{21}$ & LADBAV $^{15}$ & SUJDAD $^{35}$ & \\
DEBGOG $^{36}$ & LADQOX $^{37}$ & TUSHUL $^{38}$ & \\
\hline
\end{tabular}

Table 4.2: CSD reference codes for the $P 2_{1}$ structures compared with the XPAC method.

Reference codes in bold correspond to structures in which the $a$ axis is longer than the $c$ axis. In all cases the $b$ axis is the shortest one, with the exception of the reference code in italics (XEGTAF), which exhibits a unit cell $a=10.601(4) \AA, b=26.402(9) \AA$, $c=15.586(6) \AA, \beta=93.145(6)^{\circ}$.

\subsection{Results and discussion}

\subsubsection{Structure description}

The $\beta$-CD macrocycle does not show any remarkable distortion when compared with other structures available in the CSD. One of the primary hydroxyl groups (O6 group) is disordered between two positions 
with partial occupancies of 91 and $9 \%$, respectively. This type of disorder appears in a third of all the reported $\beta$-CD complexes. All glucose rings within the $\beta$-CD macrocycle adopt a chair conformation according to the Cremer-Pople parameters. ${ }^{39}$ The relative orientations of the glucose rings with respect to the macrocycle have been analysed in terms of angles between mean planes, in accordance with previous reports. ${ }^{10-12,12,15}$ The macrocycle is defined by the $\mathrm{O} 4$ plane and each glucose is defined by the mean plane of the atoms $\mathrm{O} 4 \_n, \mathrm{C} 4 \_n, \mathrm{C} 1 \_n$ and $\mathrm{O} 4_{-}(n+1)$. The distances between any of the $\mathrm{O} 4$ atoms and the $\mathrm{O} 4$ plane is never larger than $0.168(3) \AA$. Only glucose 3 is found to be significantly more tilted after comparison of tilting angles across all $\beta$-CD structures. All O6 groups exhibit (-)-gauche conformation, that is, pointing towards the outside of the cavity. ${ }^{2}$ A heptagon can be defined where the vertices coincide with the seven $\mathrm{O} 4$ atoms, with inner angles ranging from 120.82(9) to $136.8(10)^{\circ}$, in agreement with $128.57^{\circ}$ for the regular polygon. See Table 4.3 for all geometric parameters.

Table 4.3: Geometric parameters (calculated with PlAtON $^{40}$, in $\AA$ and ${ }^{\circ}$ ) of $\beta$-CD-DMF.

\begin{tabular}{|c|c|c|c|c|}
\hline Glucose ring & 1 & 2 & 3 & 4 \\
\hline$Q^{a}$ & $0.567(5)$ & $0.584(5)$ & $0.592(5)$ & $0.550(5)$ \\
\hline $\boldsymbol{\theta}^{b}$ & $7.4(5)$ & $4.0(5)$ & $3.1(5)$ & $5.4(5)$ \\
\hline \multirow[t]{2}{*}{ O4 torsion angles ${ }^{c}$} & $119.0(4)$ & $99.4(4)$ & $101.4(4)$ & $115.2(4)$ \\
\hline & $111.2(4)$ & $131.8(4)$ & $108.4(4)$ & $154.7(4)$ \\
\hline Tilt angle $^{d}$ & $17.3(2)$ & $5.6(2)$ & $31.8(2)$ & $9.6(2)$ \\
\hline O4 deviation ${ }^{e}$ & $-0.146(4)$ & $0.153(4)$ & $0.039(4)$ & $-0.168(3)$ \\
\hline \multirow[t]{2}{*}{ O1 torsion angles ${ }^{f}$} & $55.1(6), 168(3)$ & $50.2(5)$ & $53.5(6)$ & $51.7(6)$ \\
\hline & $-65.3(6), 48(3)$ & $-69.5(5)$ & $-65.0(5)$ & $-69.7(5)$ \\
\hline
\end{tabular}

\begin{tabular}{|c|c|c|c|}
\hline Glucose ring & 5 & 6 & 7 \\
\hline$Q^{a}$ & $0.575(5)$ & $0.554(5)$ & $0.566(5)$ \\
\hline $\boldsymbol{\theta}^{b}$ & $3.9(5)$ & $4.5(5)$ & $4.1(5)$ \\
\hline \multirow[t]{2}{*}{ O4 torsion angles ${ }^{c}$} & $102.7(4)$ & $120.2(4)$ & $104.0(4)$ \\
\hline & $123.4(4)$ & $118.6(4)$ & $142.3(4)$ \\
\hline Tilt angle $^{d}$ & $9.8(2)$ & $21.4(2)$ & $6.9(2)$ \\
\hline O4 deviation de $^{e}$ & $0.082(4)$ & $0.068(3)$ & $-0.028(4)$ \\
\hline \multirow[t]{2}{*}{ O1 torsion angles ${ }^{f}$} & $56.1(5)$ & $62.0(6)$ & $52.1(5)$ \\
\hline & $-62.4(5)$ & $-59.3(5)$ & $-68.4(4)$ \\
\hline
\end{tabular}

${ }^{a}$ Cremer-Pople puckering amplitude.

${ }^{b}$ Index to measure the deviation from the ideal chair conformation (ideal value: $\theta=0$ )

${ }^{c}$ Torsion angles C4_n-O $4 \_n-\mathrm{C} 1 \_(n-1)-\mathrm{O} 5 \_(n-1)$ and C3_n-C4_n-O $4 \_n-\mathrm{C} 1 \_(n-1)$.

${ }^{d}$ Defined as the angle formed by the $\mathrm{O} 4$ plane and the mean plane of the atoms O4_n, C4_n, C1_n and O4_ $(n+1)$.

e Distance from the atom O4_n to the O4 plane.

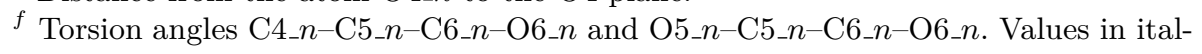
ics correspond to the minor disordered component. 
The DMF molecule is located inside the cavity of the $\beta$-CD molecule. An $\mathrm{H}$ bond (O2_2-H2O_2..O $\left.1 \_8\right)$ links a DMF molecule (H-bond acceptor) and a neighbouring $\beta$-CD related to the central host molecule by translation along the $b$ axis. The centres of mass of the host and guest molecules almost coincide and differ only by $0.977 \AA$. The DMF molecule is almost equally disordered over two positions - refined relative occupancies are 56(2) and 46(2)\%. Even allowing for some motion, as indicated by the ADPs, the carbonyl group remains in its position and acts as a pivot point.

The unit formed by $\beta$-CD and DMF is surrounded by 12.5 molecules of water. Of them, six are not disordered and show full occupancy; one (O7) has an occupancy of 50\%; two (O8 and O9) are fully occupied, but disordered between two positions each (relative occupancies of 60 and $40 \%$ and 70 and $30 \%$, respectively); the rest are unevenly distributed along two types of channels: one filled with O10 and the other filled with $\mathrm{O} 11$ and $\mathrm{O} 12$, each of them disordered between three positions (Fig. 4.1). All the water molecules are located in the space left between $\beta$-CD molecules and form a net of $\mathrm{H}$ bonds; there are no water molecules occupying the macrocycle cavity.

\subsubsection{Structure comparison}

Of the 38 structures analysed with XPAC (Table 4.2), 15 show a unit cell with dimensions $a \sim 15 \AA, b \sim 10 \AA, c \sim 21 \AA$, and $\beta \sim 110^{\circ}$. Another 19 structures are described with different settings, in which the $a$ and $c$ axes are interchanged. In addition, two other structures-DIRVOP and POVSIC - show a considerably smaller monoclinic angle of $\sim 102^{\circ}$, which is still much larger than the $90.138(3)^{\circ}$ found in the $\beta$-CD-DMF structure reported here. Another structure - XEGTAF- showing further differences in the unit-cell parameters was included in the study. XEGTAF shows a long unique axis of 26.402(9) $\AA$ and a small $\beta$ angle of $93.145(6)^{\circ}$. Since XPAC compares only the arrangements of molecules independently of the space group they crystallize in or their unit cells, consideration of these structures for the comparison is still possible and meaningful. The presence of redeterminations of the same compound accounts for 
a different number of water molecules, which may influence the crystalline packing. Variable water content is characteristic of $\beta$-CD structures, where water molecules have a high mobility thanks to flip-flop $\mathrm{H}$ bonds ${ }^{21}$.

Of all the structures considered when using a unified unit-cell setting, $\beta$-CD-DMF exhibits the longest $b$ and $c$ axes, while the $a$ axis is the shortest one. This inclusion compound also exhibits the largest unit-cell volume. For this compound, unit-cell parameters have been checked for different crystals at different temperatures; five crystals from three crystallization batches were tested and no significant variation of unit-cell parameters as a function of temperature was recorded. Full data collection was not performed and hence no comments on the possible variation of water content can be made. For most of the compounds reported up to date, a description of the crystal structure is usually limited to the identification of the characteristic herringbone packing along the unique axis. In some cases ${ }^{10,19,36}$ the position of a $\beta$-CD unit is given relative to the unit-cell axes. The absence of an in-depth structure comparison has motivated the XPAC analysis presented here.

Table 4.4 shows a summary of the results of the XPAC analysis (for complete information refer to the supplementary material in R. GraneroGarcía amd F. P. A. Fabbiani, Acta. Cryst., 2014, B70, 586-594). The following terminology is introduced to accompany the interpretation of the results (Fig. 4.2). A strand defines a linear sequence of single $\beta$-CD molecules. A strand can be stacked along the $b$ axis or side-to-side along any of the other two axes, depending on the settings. A herringbone refers to two neighbouring stacked strands related by the screw operation along the $b$ axis. A plane consists of several parallel herringbones. The full structure is formed when several planes are stacked one on top of each other in the third crystallographic dimension. A similarity with three dimensions implies that the two structures match at the level of full structures. Two-dimensionality means that only planes match between the two structures. One-dimensionality refers to a match of herringbones or strands only. Zero-dimensionality implies that the match is reduced to isolated molecules, although zero-dimensionality has also been used in 
the literature to refer to a finite unit comprising two or more molecules.

All structures are characterized by the herringbone-type packing, as confirmed by inspection with any structure viewing program. The degree of similarity between structures can, however, be quantified further. On the basis of the XPAC calculations, the following observations can be made:

i In most cases packing differences are translated into substantial differences in the unit-cell parameters. There are exceptions to this: for DEWCOY, KOBRIC, or UPULEX the unit cells do not show any differences and the packing is still distinct (zero-dimensional similarity).

ii DIRVOP and POVSIC, which exhibit the shortest among the longest axis—20.118(2) and 20.042(6) $\AA$, respectively, against the mean value of 21.0(9) $\AA$ —and different $\beta$ angles-102.14(1) and 102.30(2) ${ }^{\circ}$, respectively, against a mean of $108(5)^{\circ}$ — exhibit three-dimensional similarity with each other (dissimilarity index of 1.9), two-dimensional similarity with $\beta$-CD-DMF, zero-dimensional similarity with DEWCOY, KOBRIC, UPULEX, and XEGTAF, and one-dimensional similarity with the remaining 31 structures.

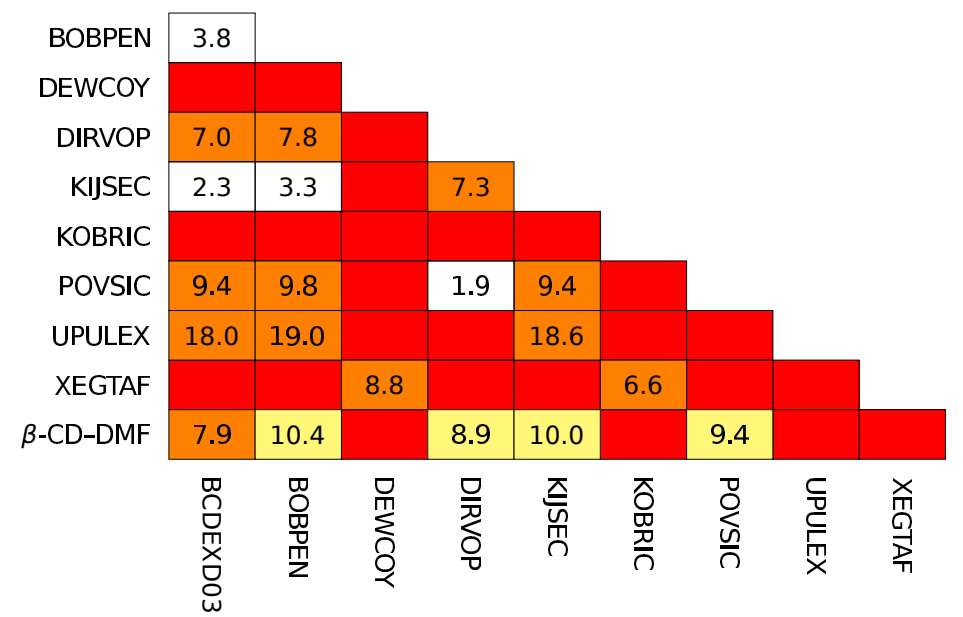

Table 4.4: Selected results from the comparisons performed with XPAC.

The dimensionality of structural similarity is colour coded in white (threedimensional), yellow (two-dimensional), orange (one-dimensional) or red (zerodimensional). The dissimilarity index is indicated for each comparison, except for the zero-dimensional cases, where no dissimilarity index is ouput. Large dissimilarity indices (like those for UPULEX) point to significant geometrical differences in the arrangement of $\beta$-CD molecules that are nevertheless still consistent with the overall herringbone-packing arrangement. Only results described in detail in $\S 4.3 .2$ of the text are shown. 
iii The one-dimensional match for DIRVOP always corresponds to a strand, while for povsic it is extended to a herringbone for 18 of the 31 structures. A visual inspection of the compared structures indicates that the herringbone motif is always preserved, but the distance between stacked strands can be different, increasing the dissimilarity. This could be ascribed to the nature of the guest. The guests molecules in DIRVOP and POVSIC are rather small molecules (hexamethylenetetramine and trans-cyclohexane-1,4-diol, respectively), which are unusually located towards the opening of the $\beta$-CD near the secondary alcohol groups (usually the guest is displaced towards the other side). A similar displacement of the guest is found in other structures (e.g. NUfVIU, PIJGIY, and PIJGOE), yet these do not show any significant packing differences. These observations point out, in contrast to previous reports, ${ }^{12}$ that a guest that is fully included in the cavity has no direct effect on the herringbone packing. It is rather the formation of the H-bonded network, and hence the location of hydration water molecules, which ultimately determines the separation between neighbouring herringbones. Previous studies proposed that the formation of $\mathrm{H}$-bonded networks in $\beta$-CD complexes is highly influenced by the guest and that guests that are similar in size and shape can lead to unrelated H-bonded networks, unit cells and space groups. ${ }^{24,41}$ This idea is supported by observations on XEGTAF (see vi).

Figure 4.2: Schematic explanation of terminology used in the text. The strand corresponds to the motif enclosed in the red area, the herringbone corresponds to the blue area and the plane to the green area.

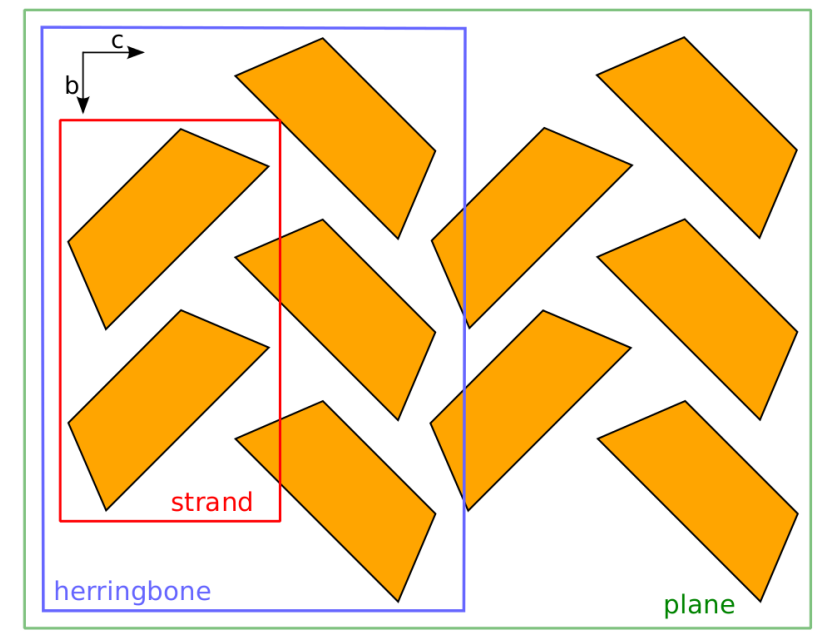


iv One-dimensional similarity is observed between UPULEX and most of the structures. However, the reasons for the one-dimensional similarity are of a different nature compared with DIRVOP and POVSIC. This is confirmed by the fact that pairs UPULEX-DIRVOP and UPULEXPOVSIC show zero-dimensional similarity. The reason is a different tilt of the $\beta$-CD molecule with respect to the unit-cell axes.

v Amongst structures with similar unit-cell parameters, DEWCOY and KOBRIC show zero-dimensional similarity with all other structures with the exception of XEGTAF (two-dimensional similarity), despite XEGTAF's long monoclinic axis. In the case of DEWCOY, the match is through a stacked strand along the shortest axis, while for KOBRIC it is a side-to-side strand. This exceptional difference might be produced by a different type of guest, which is $\mathrm{KOH}$ in KOBRIC. $\mathrm{K}^{+}$ is not included in the cavity. All other structures, with the exception of BOBPEN, have neutral molecules as guests. In BOBPEN, the guest is $\mathrm{HI}$, but $\mathrm{I}^{-}$is located inside the cavity, emulating the organic inclusion complexes, and it does not exhibit differences in packing. This explanation does, however, not hold for DEwCOY, which shows a rather big guest partially outside the cavity. Other structures have this same feature and show no difference in packing.

vi XEGTAF, which has a different unit cell, does only match with DEWCOY and KOBRIC (one-dimensional similarity). In this case the guest is quite large so that approximately half of it is located outside the cavity, resulting in the elongation of the $b$ axis. These observations were reported originally by Fan et al. ${ }^{26}$ and a similar explanation was given for the elongation of the $b$ axis in KOBRIC. ${ }^{10}$

vii The results of the packing similarity analysis of the $\beta$-CD-DMF structure can be partially interpreted using a combination of the observations i-vi above. The structure exhibits the smallest $\beta$ angle, $90.138(3)^{\circ}$, the shortest among the short axes, 9.794(2) $\AA$, and also the shortest among the middle-length axes, 15.500(3) $\AA$. This is the only case where differences in terms of unit-cell parameters are found in axes that are not the longest one. The XPAC method determines that the packing similarity for the $\beta$-CD-DMF complex with most 
of the structures is one-dimensional, in agreement with the observations for the other two cases with a different $\beta$ angle (DIRVOP and POVSIC). It shows zero-dimensional similarity with the previously described outliers (DEWCOY, KOBRIC, UPULEX and XEGTAF), in line with the considerations for POVSIC and DIRVOP (see ii and iii). Indeed the match with both POVSIC and DIRVOP is two-dimensional. Two-dimensional similarity is also observed with BOBPEN and KIJSEC; however, we could not find an obvious explanation for this.

\subsection{Conclusions and outlook}

A new complex of $\beta$-CD with DMF has been crystallized and its structure determined by single-crystal X-ray diffraction. The structure exhibits the herringbone-type packing that appears frequently for these complexes, but has significant differences in both the unit-cell parameters and the relative distances and angles of the packed $\beta$-CD molecules.

A detailed comparison among 38 structurally related $\beta$-CD complexes has been carried out using the XPAC method in an attempt to find the origin of the packing differences and similarities. It is now clear that the herringbone packing is maintained in all the structures and differences arise from the relative orientation of $\beta$-CD molecules in the herringbone motif, that is, along the longest axis, but never in the other two (with the exception of XEGTAF). However, it cannot be said that differences in packing are always reflected in different unit-cell parameters, whereas the opposite is always true. The conformation of the $\beta$-CD molecule is not significantly different in the crystal structures considered in this study; hence differences in packing are independent of $\beta$-CD geometry.

The results of the XPAC analysis also confirm previous observations that the guest molecule does not directly influence the crystal packing, since differences in guest type and position within the cavity appear in structures with both zero- and three-dimensional similarity. It seems that the position of water molecules and the H-bonded network, which might also be influenced by the H-bonding capability of the guest, play 
an important structure-directing role. Such an analysis has not been carried out in this study, but this theory could be tested in the future. In this context, Steiner et al., based on the observation that the position of hydration water molecules located outside the cavity of the $\beta$-CD molecule is invariant in six structures, reported that it is the packing that determines the position of the water molecules. ${ }^{33} \mathrm{~A}$ similar observation was reported by Gessler et al.; however, in this case it was found that the guest molecule is likely to be located in such a way that possible $\mathrm{H}$-bond donor or acceptor atoms are located in the same position than the water molecules displaced during the inclusion process. ${ }^{25}$ Fan et al. have also reported that structural characteristics of $\beta$-CD inclusion complexes arise from a relation between guest/solvent type and position, and H-bonded network formation. ${ }^{26}$ Rationalisation of the structure complexity of $\beta$-CD inclusion complexes continues to be an area of intense research activity. 


\section{References}

[1] D. Mentzafos, I. M. Mavridis, G. Le Bas and G. Tsoucaris, Acta Cryst., 1991, B47, 746-757.

[2] W. Saenger and T. Steiner, Acta Cryst., 1998, A54, 798-805.

[3] T. Gelbrich and M. B. Hursthouse, CrystEngComm, 2005, 7, 324336.

[4] E. Egert and G. M. Sheldrick, Acta Cryst., 1985, A41, 262-268.

[5] G. M. Sheldrick, Acta Cryst., 2015, C71, 3-8.

[6] C. B. Hübschle, G. M. Sheldrick and B. Dittrich, J. Appl. Cryst., 2011, 44, 1281-1284.

[7] G. M. Sheldrick, SADABS v2008-1, Bruker-AXS, Madison, Wisconsin, USA, 2008.

[8] F. P. A. Fabbiani, B. Dittrich, A. J. Florence, T. Gelbrich, M. B. Hursthouse, W. F. Kuhs, N. Shankland and H. Sowa, CrystEngComm, 2009, 11, 1396-1406.

[9] K. Lindner and W. Saenger, Carbohydr. Res., 1982, 99, 103-115.

[10] P. Charpin, I. Nicolis, F. Villain, C. de Rango and A. W. Coleman, Acta Cryst., 1991, C47, 1829-1833.

[11] M. M. Pop, K. Goubitz, G. Borodi, M. Bogdan, D. J. A. De Ridder, R. Peschar and H. Schenk, Acta Cryst., 2002, B58, 1036-1043.

[12] T. Aree and N. Chaichit, Carbohydr. Res., 2002, 337, 2487-2494.

[13] T. Aree and N. Chaichit, Carbohydr. Res., 2008, 343, 2285-2291.

[14] T. Aree, B. Schulz and G. Reck, J. Incl. Phenom. Macro., 2003, 47, 39-45.

[15] A. Paulidou, K. Yannakopoulou and I. M. Mavridis, J. Incl. Phenom. Macro., 2010, 68, 297-303.

[16] E. J. Wang, Z. X. Lian and J. Cai, Carbohydr. Res, 2007, 342, $767-771$.

[17] M. Crisma, R. Fornasier and F. Marcuzzi, Carbohydr. Res., 2001, 333, $145-151$.

[18] P. Guo, Y. Su, Q. Cheng, Q. Pan and H. Li, Carbohydr. Res., 2011, 346, 986-990.

[19] K. Harata, Bull. Chem. Soc. Jpn., 1984, 57, 2596-2599. 
[20] T. Aree and N. Chaichit, Supramol. Chem., 2009, 21, 384-393.

[21] T. Steiner and G. Koellner, J. Am. Chem. Soc., 1994, 116, 51225128.

[22] J. L. Wang, F. M. Miao, W. H. Zhou and S. K. Ma, Chin. J. Chem., 2002, 20, 358-361.

[23] Y. Zhang, S. Yu and F. Bao, Carbohydr. Res., 2008, 343, 25042508.

[24] Y. M. Zhang, Z. X. Yang, Y. Chen, F. Ding and Y. Liu, Cryst. Growth Des., 2012, 12, 1370-1377.

[25] K. Gessler, T. Steiner, G. Koellner and W. Saenger, Carbohydr. Res., 1993, 249, 327-344.

[26] Z. Fan, C. Diao, H. Song, Z. Jing, M. Yu, X. Chen and M. Guo, J. Org. Chem., 2006, 71, 1244-1246.

[27] M. K. Grachev, I. A. Senyushkina, G. I. Kurochkina, K. A. Lysenko, L. K. Vasyanina and E. E. Nifant'ev, Russi. J. Org. Chem., 2010, 46, 1506-1510.

[28] T. Steiner, G. Koellner, K. Gessler and W. Saenger, J. Chem. Soc., Chem. Commun., 1995, 511-512.

[29] Y. V. Lisnyak, A. V. Martynov, V. N. Baumer, O. V. Shishkin and A. V. Gubskaya, J. Incl. Phenom. Macro., 2007, 58, 367-375.

[30] K. Lindner and W. Saenger, Carbohydr. Res., 1982, 107, 7-16.

[31] L. Damodharan, V. Pattabhi and K. Nagarajan, Mol. Cryst. Liq. Crys., 2004, 423, 17-35.

[32] T. Steiner and W. Saenger, J. Chem. Soc., Chem. Commun., 1995, 2087-2088.

[33] T. Steiner, G. Koellner and W. Saenger, Carbohydr. Res., 1992, 228, 321-332.

[34] T. Steiner and W. Saenger, J. Chem. Soc., Perkin Trans. 2, 1998, 2, 371-378.

[35] R. W. Seidel and B. B. Koleva, Acta Cryst., 2009, E65, o3162o3163.

[36] K. Harata, K. Uekama, M. Otagiri, F. Hirayama and Y. Ohtani, Bull. Chem. Soc. Jpn., 1985, 58, 1234-1238. 
[37] G. Kurokawa, M. Sekii, T. Ishida and T. Nogami, Supramol. Chem., 2004, 16, 381-384.

[38] J. Y. Li, D. F. Sun, A. Y. Hao, S. H. Y. and S. J., Carbohydr. Res., 2010, 345, 685-688.

[39] D. Cremer and J. A. Pople, J. Am. Chem. Soc., 1975, 97, 13541358.

[40] A. L. Spek, Acta Cryst., 2009, D65, 148-155.

[41] Y. L. Zhao, D. Benítez, I. Yoon and J. F. Stoddart, Chem. Asian J., 2009, 4, 446-456. 


\section{Hydration of $\boldsymbol{\alpha}$-cyclodextrin at}

\section{high pressure}

\subsection{Introduction}

$\alpha$-CD hydrates have been the object of extensive research; four different forms (named I to IV) have been characterised over the course of 25 years. All of them belong to the space group $P 2_{1} 2_{1} 2_{1}$ and their unit cells, although distinct, hold a reasonable resemblance. In spite of this, the four forms exhibit three different crystal packings (forms I and III exhibit the same packing arrangement) and slightly different conformations of the macrocycle. This latter feature was revealed to be the key factor in determining the complex-formation capabilities of $\alpha$-CD.

The structure of form I was originally reported by Manor and Saenger in $1974,{ }^{1}$ and subsequently redetermined by neutron diffraction by Klar et al. ${ }^{2}$ This form is easily obtained by recrystallisation from a saturated aqueous solution and is characterised by the herringbone packing motif. ${ }^{3}$ In form I, $\alpha$-CD exists as an hexahydrate, with two water molecules 
crystallising inside the macrocycle cavity and four in the intermolecular space.

What set this structure apart from any other CD structure known at the time was a different conformation of the $\alpha$-CD molecule (Fig. 5.1). $\mathrm{CD}$ molecules in all other $\alpha$ - and $\beta$-CD complexes, including hydrates of the latter, exhibit a similar conformation, in which the macrocycle is round and all the glucose rings are equaly tilted with respect to the equatorial plane. One of the glucose rings of $\alpha$-CD in form I is considerably more tilted than the rest, which causes the macrocycle to adopt a tense conformation. ${ }^{1,4}$ This tense conformation was deemed as a high-energy state, resembling the conformation of $\alpha$-CD in aqueous solution. In the solid state the conformation is stabilised by the formation of $\mathrm{H}$ bonds between two of the primary hydroxy groups (O6 groups) and the water molecules inside the cavity, which requires said groups to be facing towards the cavity - $(+)$-gauche conformation - a situation energetically less favourable than facing outwards-(-)-gauche conformation. ${ }^{5}$ Additionally, the distance between secondary hydroxy groups (O2 and O3) is larger in $\alpha$ - than in $\beta$-CD, which weakens the extended $\mathrm{H}$ bonded network of the $\alpha$-CD structure, ${ }^{6}$ allowing for the observed distortion.

Figure 5.1: Molecule of $\alpha$-CD in its tense conformation from the structure of form I at $0.20 \mathrm{GPa}$ (\$5.3.1). Water molecules outside the cavity and $\mathrm{H}$ atoms have been removed for clarity. The two O6 groups in the $(+)$-gauche conformation have been labelled; O6_1 adopts both $(+)$ and $(-)$ conformations with a $50 \%$ probability. Possible $\mathrm{H}$ bonds between $\mathrm{O} 6$ groups and the water molecules inside the cavity have been plotted as blue dashed lines. It can be seen how glucose ring 5 is tilted with respect to the rest of the glucose rings.

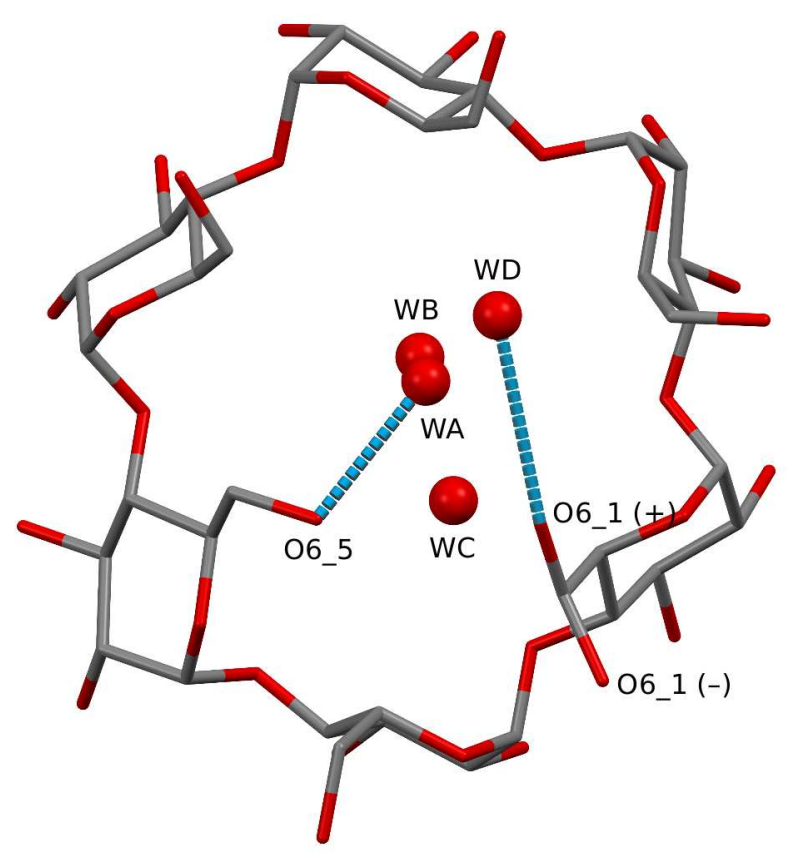


Form $\mathrm{II}^{7}$ co-precipitates with form I, albeit it only represents a $2 \%$ of the crystallisation outcome and its formation is not well understood. It is also an hexahydrate, although the distribution of the water molecules is different, namely five molecules are located outside the cavity and one inside. In the absence of other guests the cavity is partially filled by an O6 group from a neighbouring molecule, which is made possible via a distortion of the herringbone packing. Despite the changes in the packing motif, the conformation of the $\alpha$-CD molecules is nearly identical in both forms I and II.

Form III ${ }^{5}$ was obtained while attempting to crystallise a complex of $\alpha$-CD with barium cations, which instead led to a 7.25-hydrate. In this structure, there are five water molecules in the intermolecular spaces and 2.57 inside the cavity of the macrocycle. In this case, the herringbone packing is identical to the one observed in form I, but the conformation of the $\alpha$-CD molecule differs, as it is round in form III, with all the O6 groups in (-)-gauche conformation.

The last form, IV ${ }^{8}$ was obtained in a similar manner than form III, while trying to crystallise complexes of $\alpha$-CD with organic molecules containing alkylic chains. The stoichiometry corresponds to an 11-hydrate, with up to 7 molecules inside the cavity, a value which is very likely to be overestimated. The conformation of the $\alpha$-CD molecules in form IV is also round, although the packing is of the brick type, unrelated to the herringbone type observed in the other three forms. ${ }^{3}$

The distinct conformation of $\alpha$-CD in the hydrate forms I and II was used by Saenger et al. to explain inclusion-complex formation in $\alpha$-CD by the induced-fit mechanism. ${ }^{4}$ According to this mechanism, characteristic of enzymatically-catalysed reactions, ${ }^{9} \alpha$-CD exists in solution in its tense form, with a low occupancy of the cavity, similar to the one observed in crystal structures. In the presence of a guest of suitable size, water, which is not fully coordinated by $\mathrm{H}$ bonds, easily leaves the cavity allowing the formation of an inclusion complex. The existence of both forms III and IV confirms the induced-fit mechanism, as the $\alpha$-CD molecule in the round shape filled with more than two water molecules is a likely intermediate state during the complexation process. 
The induced-fit mechanism explains how inclusion takes place, but not why; the driving forces of the inclusion process have been matter of a thourough debate. ${ }^{10}$ Van der Waals and hydrophobic interactions seem to be the major driving forces, while electrostatic interactions and hydrogen bonding mainly affect the final conformation of the complex.

Prior to the commencement of the work reported here, we showed that by recrystallising $\alpha$-CD form I at $0.65 \mathrm{GPa}$ it was possible to increase the number of water molecules inside the cavity by 0.8 , similarly to what is observed in form III, albeit while retaining the tense conformation of the macrocycle. Such structure had been previously predicted by computational means. ${ }^{11}$ The inclusion of extra water was accompanied by a change in the conformation of an O6 group, from 100\% (+) to $50: 50 \%(+):(-)$, and in turn by a noticeable increase of the solventaccessible volume of the $\alpha$-CD cavity. This chapter presents a deeper crystallographic insight into the compression of $\alpha$-CD form I, showing that the inclusion of additional water can occur in the solid state, without the need of recrystallisation, and attempts to provide a new mechanism for the inclusion of water by means of MD simulations.

\section{$5.2 \quad$ Experimental}

\subsubsection{Crystal growth and compression study}

Crystals of hydrate form I of $\alpha$-CD were first grown at ambient conditions by slow evaporation of a saturated aqueous solution of $\alpha$-CD

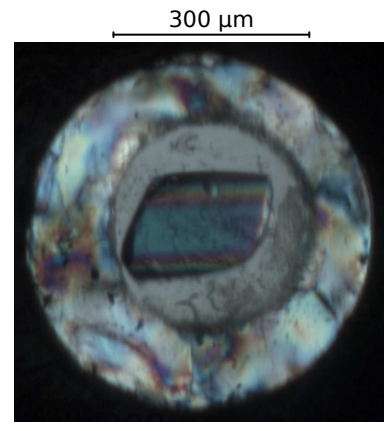

Figure 5.2: Single crystal of $\alpha$-CD form I at $0.20 \mathrm{GPa}$.
(Sigma-Aldrich, used as received; solubility at $298 \mathrm{~K}$ is $145 \mathrm{mg} / \mathrm{mL}$ ). A crystal grown under these conditions was loaded into a $90 / 100^{\circ} \mathrm{DAC}$ (see Appx. I.3 for a technical description of the cell and further information on its use) along with its saturated solution and directly compressed to $0.20 \mathrm{GPa}$. The DAC was equipped with a brass gasket of thickness $200 \mu \mathrm{m}$ pre-indented to $120 \mu \mathrm{m}$ and with a hole $300 \mu \mathrm{m}$ in diameter. $\alpha$-CD exhibits a similar behaviour than $\beta$-CD, dissolving upon increasing pressure (§3.2.1); ${ }^{12}$ although dissolution occurs as early as $0.20 \mathrm{GPa}$, the final size of the crystal was acceptable for data collection (Fig. 5.2). 
The pressure range in which hydration takes places - from 0.0001 to $0.65 \mathrm{GPa}$ - has been explored using three different crystals, measured at 0.0001, 0.20, and 0.65 $\mathrm{GPa}^{12}$ (named St1, St2, and St3, respectively). To ensure that the differences observed between the lattice parameters of the three specimens are within the limits of reasonable experimental error, a compression study of $\alpha$-CD was also performed. A triangular DAC with $800 \mu \mathrm{m}$ diamond culets was used for this experiment (Appx. I.2). The cell was equipped with an Inconel 718 gasket pre-indented to a thickness of $160 \mu \mathrm{m}$ and sample chamber $495 \mu \mathrm{m}$ in diameter. Compression studies are usually performed by collecting X-ray diffraction data at different points on increasing pressure, but due to the dissolution of $\alpha$-CD, the study was performed on decompression. The DAC was loaded with a crystal grown at ambient pressure, as described above, together with the saturated aqueous solution and pressure was increased to 0.60 GPa; complete dissolution of the crystal was observed at $0.40 \mathrm{GPa}$. Polycrystalline material was obtained on decompression and a single crystal suitable for diffraction was obtained at $0.54 \mathrm{GPa}$ following the usual procedure of temperature cycling (§1.1.2). Data were collected on said specimen, and subsequently pressure was slowly released, collecting data at $0.40,0.33$, and $0.10 \mathrm{GPa}$.

\subsubsection{X-ray data collection and processing}

Data on the crystal at 0.20 GPa were collected at beamline F1 at Doris (DESY) on a Huber four-circle diffractometer, with a combination of $0.5^{\circ}$ $\omega$ and $\phi$ scans at two different values of $\chi\left(0\right.$ and $\left.120^{\circ}\right)$. Radiation of $0.5 \AA$ with a beam $1 \times 1 \mathrm{~mm}$ was used, exposing each frame for $40 \mathrm{~s}$. The detector was a MAR165 CCD. Information in frame headers were updated with CMH and frames were converted from MAR to Bruker-Nonius Smart format using M2B. ${ }^{13}$ A final conversion to SFRM format was performed with the tools included in Apex2. ${ }^{14}$

Data for the 0.0001 and $0.65 \mathrm{GPa}$ pressure points come from two further crystal specimens which had been measured previously, as explained above, and the resulting structures published. ${ }^{12}$ 
Data for the compression study were collected using a MAR345 image plate and an InCOATEC microfocus $(\mathrm{I} \mu \mathrm{S})$ source of $\mathrm{Ag}$ radiation $(0.56085 \AA)$. Each collection consisted of $0.5^{\circ} \phi$ scans with $60 \mathrm{~s}$ exposure time. The setup of this diffractometer only allowed for $\phi$ scans, which was compensated by recollecting data with the DAC rotated $120^{\circ}$ around the axis of the beam. MAR frames were directly converted to SFRM format using APEx2.

Data were integrated following the strategy outlined in $§ 1.1 .3$.

\subsubsection{Structure refinement of $\alpha$-cyclodextrin hydrate at $0.2 \mathrm{GPa}$}

At $0.2 \mathrm{GPa} \alpha$-CD does not undergo any major structural transformation apart from an increase in hydration number, hence a low-temperature determination of form I (CSD reference code: CHXAMH04 ${ }^{12}$ ) has been used as starting point for refinement. Full-matrix least-squares refinement against $F^{2}$ has been carried out using SHELXL v2014/7, ${ }^{15}$ through the GUI SHELXLE. ${ }^{16}$

All non-H atoms were refined anisotropically, with the exception of three out of eight water molecules, for which no $\mathrm{H}$ atoms could be determined. Two of these three water molecules have an occupancy of $20 \%$. Occupancy factors were initially refined and then fixed to the refined values. All bond distances and angles were restrained to target values automatically generated on the Grade Web Server. ${ }^{17}$ Torsion angles were restrained in a similar manner, excluding those involving $\mathrm{O}$, which are known to be conformationally flexible. In addition, the geometry of all the glucose rings was restrained to be the same. $\alpha$-CD $\mathrm{H}$ atoms were initially placed geometrically and constrained using the riding model; some positions were later improved by looking at difference Fourier maps. $\mathrm{H}$ atoms belonging to water molecules were located in Fourier difference maps when possible, their positions optimized according to the formation of possible $\mathrm{H}$ bonds and then refined subject to restraints. For further crystallographic data see Table 5.1. 


\begin{tabular}{ll}
\hline Crystal data & \\
\hline Chemical formula & $\mathrm{C}_{36} \mathrm{H}_{60} \mathrm{O}_{30} \cdot 6.4 \mathrm{H}_{2} \mathrm{O}$ \\
Molecular mass $\left(\mathrm{g} \mathrm{mol}^{-1}\right)$ & 1088.14 \\
Temperature $(\mathrm{K})$ & $298(2)$ \\
Pressure $(\mathrm{GPa})$ & $0.20(5)$ \\
Crystal system, space group & orthorhombic, $P 2_{1} 2_{1} 2_{1}$ \\
$a, b, c(\AA)$ & $14.8069(13), 33.970(3), 9.4140(8)$ \\
$V\left(\AA^{3}\right)$ & $4735.2(7)$ \\
$Z$ & 4 \\
Crystal size $(\mathrm{mm})$ & $\sim 0.20 \times 0.15 \times 0.10$ \\
\hline Data collection & \\
\hline Radiation wavelength $(\AA)$ & 0.5000 \\
Absorption correction & $\mathrm{Semi}-\mathrm{empirical}(\mathrm{using} I$ meas. $)$, SADABS ${ }^{18}$ \\
$T_{\text {min }}, T_{\text {max }}$ & $0.5898,0.7440$ \\
Number of $[I>2 \sigma(I)]$ reflections: & \\
- measured & 35544 \\
- independent & 3157 \\
- observed & 2913 \\
$(\sin \theta / \lambda)_{\text {max }}\left(\AA^{-1}\right)$ & 0.556 \\
$R_{\text {int }}(\%)$ & 4.65 \\
\hline Refinement & \\
\hline$R\left[F^{2}>2 \sigma\left(F^{2}\right)\right](\%)$ & 3.57 \\
$w R\left(F^{2}\right)(\%)$ & 9.08 \\
$S$ & 1.03 \\
Number of reflections & 3157 \\
Number of parameters & 1797 \\
Number of restraints & 691 \\
$\Delta \rho_{\min }, \Delta \rho_{\max }\left(\mathrm{e} \AA{ }^{-3}\right)$ & $-0.205,0.313$ \\
\hline
\end{tabular}

Table 5.1: Crystallographic data and refinement parameters of $\alpha-\mathrm{CD} \cdot 6.4 \mathrm{H}_{2} \mathrm{O}$.

\subsubsection{Molecular dynamics}

For a description of fundamental concepts in $\mathrm{MD}$, refer to $\S 1.2$; the present section contains only technical details on how the simulations were implemented. All the simulations in this chapter use the CHARMM (Chemistry at HARvard Macromolecular Mechanics) force field. ${ }^{19,20}$ Water molecules, either in the lattice or the bulk of the solvent, were parametrised as TIP3P. ${ }^{21}$ The force field has been used without any modification of atomic charges or force constants. The same force field has been used to minimise the energy of all the molecular systems prior to simulation. All simulations in this chapter have been performed using NAMD (v2.9). ${ }^{22}$

\subsubsection{Equilibrations of infinite crystals}

These simulations are simple equilibrations of an infinite lattice of $\alpha$-CD molecules (that is, without bulk solvent) let to evolve at constant pres- 
sure values. Four different equilibrations have been carried out, by simulating two different setups at two different pressures each. The two setups are termed AP and HP and are based on the crystal structures at 0.0001 and $0.65 \mathrm{GPa}$, respectively.
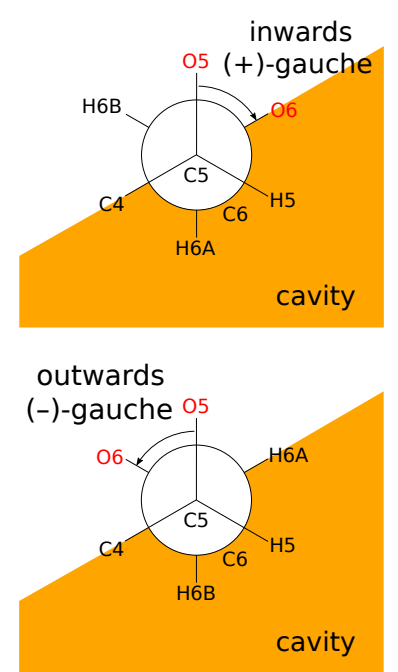

Figure 5.3: Definition of the $(+)$ - and (-)-gauche conformations of the O6 groups in CD molecules.

The nearly-cubic simulation box of the AP setup consists of six unit cells (two replicas on the $a$ axis, one on $b$, and three on $c$ ) of the AP crystal structure of $\alpha$-CD form I (CSD reference code: CHXAMH02 ${ }^{2}$ ). According to the crystal structure, O6_1 can adopt both (+)- and (-)-gauche conformations (Fig. 5.3); the likelihood of the latter being only $10 \%$. Such an small disorder was deemed to have no effect in the outcome of the simulation; hence it was not modelled in the setup. Lattice water in the crystal structure has been included in the setup; there are six water molecules per $\alpha$-CD molecule, two (termed WA and WB) located inside the cavity of the macrocycle and four (termed $\mathbf{W} \mathbf{1}, \mathbf{W} \mathbf{2}, \mathbf{W} 3$, and W4) in intermolecular spaces.

$\alpha$-CD form I at $0.65 \mathrm{GPa}$ also exhibits disorder of the O6_1 group, with the $(-)$ and $(+)$ conformations equally populated. Furthermore, there are two additional lattice water molecules per $\alpha$-CD molecule in the structure (termed $\mathbf{W C}$ and $\mathbf{W D}$ ), located inside the $\alpha$-CD cavity with an occupancy of only $40 \%$ each. The six water molecules W1-4, WA, and WB are also present in this structure at the same positions. The disorder is what distinguishes the HP structure from the AP one, hence it must be modelled in the setup. Because NAMD does not allow to model disorder by means of percentage populations in a unit cell, and rather requires fully occupied atoms which might or not be present in certain unit cells within a super cell, the simulation box of the HP setup was required to be larger than that of the AP setup (smallest setups were preferred whenever possible to reduce the computational cost of the calculations). The simulation box of the HP setup is composed of ten unit cells (two replicas on the $a$ axis, one on $b$, and five on $c$ ) of the HP structure of $\alpha$-CD form I determined at $0.65 \mathrm{GPa}$ (CSD reference code: REGPAW $^{12}$ ), which accounts for $40 \alpha$-CD molecules and 320 water positions, 240 of which are fully occupied (W1-4, WA, and WB) and 80 partially filled with only 32 water molecules (WC and WD). By 
random selection, the conformation of the $06-1$ group was made $(+)$ in half the $\alpha$-CD molecules and (-) in the other half. From the latter, 16 positions WC and 16 WD (out of 20 each) were filled with water. This distribution of disordered features is in agreement with the hypothesis that the O6_1 needs to change from the $(+)$ to the $(-)$ conformation for the additional water to access the cavity of the macrocycle. For a complete description on how the disorder has been reproduced, see Appx. F.

Both setups were simulated in the NPT ensemble at constant pressures of 0.0001 and $0.65 \mathrm{GPa}$. The simulation of each setup as its respective pressure, $\mathrm{AP}$ at $0.0001 \mathrm{GPa}$ and $\mathrm{HP}$ at $0.65 \mathrm{GPa}$, are intended to reproduce the behaviour of the crystal in real conditions. The other two simulations, with the pressures interchanged, allow to control for model bias (§5.3.2.1). Further details on how the simulations were performed can be found in Table 5.2 .

\begin{tabular}{ll}
\hline Simulation length & $100 \mathrm{~ns}$ \\
Time step & $1 \mathrm{fs}$ \\
Frame output & every 2000 steps $(2 \mathrm{ps})$ \\
Number of frames & 50000 \\
Minimisation & 100 steps $(0.1 \mathrm{ps})$ \\
Force field & CHARMM \\
PBC & yes \\
Ensemble & $N P T$ \\
Temperature control & Langevin \\
Temperature & $298 \mathrm{~K}$ \\
Pressure control & Langevin (not flexible) \\
Pressure & 0.0001 or $0.65 \mathrm{GPa}$ \\
Treatment of interactions & \\
- Covalent & first and second neighbours \\
- Mixed & fourth neighbours \\
- Coulomb and Van der Waals & fifth and higher neighbours (PME) \\
Interactions cutoff & $12 \AA$ \\
Smoothing after cutoff & $10 \AA$ \\
Treatment of bonds with H atoms & rigid \\
\hline
\end{tabular}

Table 5.2: Details of the equilibrations carried out on infinite crystals.

The trajectories obtained from these simulations serve a double purpose. On the one hand, they are simple enough to test the performance of the force field at the simulation conditions. On the other hand, they provide and insight on the pressure dependence of the dynamics of the O6_1 group in the absence of bulk solvent.

A Python script has been written to analyse the evolution of the disorder of the $\mathrm{O} 6$ groups during the trajectories. Although the group 
of interest is $06 \_1$, the analysis was applied to all O6 groups, to ensure that the entire structure was properly reproduced by the simulation. The script loops over all frames, identifying all $\mathrm{O} 6$ groups of all $\alpha$-CD molecules in a frame and establishing their conformations, either $(+)$ or (-)-gauche by means of the torsion angle around the bond $\mathrm{C} 5-\mathrm{C} 6$. (Fig. 5.3).

Once the conformation of all O6 groups is known, a histogram of the torsion angles is created for each group in the simulation box to identify the equilibrium positions, which correspond to the maxima of the histograms. Apart from the expected $(+)$ and $(-)$ conformations, a third local maximum is seen in many histograms and was identified as an intermediate state in the conformational change between $(+)$ and $(-)$, based on shorter residence times (evaluated visually as area of the histogram). Each equilibrium position of each O6 was defined by the mean value of the torsion angle plus and minus $3 \sigma$ calculated from a normal curve fitted to the corresponding maximum of the histogram. The script loops over the complete list of torsion angles, assigning them to the corresponding conformation of each O6 group. Torsion angles outside equilibrium positions are rejected as outliers - they correspond to transition conformations. The total residence time of each O6 group in each conformation can be calculated from the number of torsion angles assigned to it. Finally, residence times in each conformation of O6 groups corresponding to the same glucose ring of all $\alpha$-CD molecules in the simulation box were combined and expressed as percentages, which is equivalent to the usual expression of disorder in crystal structures (space and time average).

The script can function in an entirely automatic way, but graphic output is provided to permite user input in cases where more than three maxima existed in the histogram. Nevertheless, such cases were rare and statistically not significant.

\subsubsection{Equilibrations of molecular clusters in water}

These equilibrations follow the same pattern described in the previous section: two setups, AP and HP, simulated both at 0.0001 and $0.65 \mathrm{GPa}$, 
although the setups employed were different. The boxes in this set of simulations consisted of a finite cluster of $\alpha$-CD molecules submerged in liquid water, in an attempt to replicate the behaviour of a crystal surrounded by bulk solvent. PBC were still in use to avoid surface effects on the borders of the box. ${ }^{23}$ The molecular clusters need to be larger than the ones described in the previous section, to accurately describe the properties of the crystal, while still keeping the amount of molecules to a minimum. Both AP and HP clusters were built from the setups described in the previous section, by doubling the number of unit cells repeated along the $a$ axis from two to four. Such clusters contain at least four layers of $\alpha$-CD along each axis; in this way there is a surface of $\alpha$-CD molecules surrounding a small core of two layers of molecules, which cannot be directly influenced by the solvent. Although much larger clusters would be desirable, these were not feasible due to computational limitations. The disordered features of the HP cluster were remodelled, following the same criteria outlined previously, to ensure a random distribution over the entire cluster (Appx. G).

Each cluster was completely surrounded by a $12 \AA$-thick layer of water, containing over 29000 water molecules. The simulations were performed as described in Table 5.2, although the minimisation time was increased to $20 \mathrm{ps}$ (the system used in the previous section was already close to the energy minimum, while that is not the case here) and the simulation length was limited to $50 \mathrm{~ns}$ (to reduce consumption of computational resources), producing only 25000 frames.

The aim of these simulations was to study the effects of hydrostatic pressure applied on the crystal via bulk solvent. By analysing the disorder of the O6 groups and comparing the results with those obtained from the crystal without bulk solvent, it would be possible to determine whether the disorder is caused by pressure alone or if it requires the presence of solvent. Furthermore, inclusion events, that is, water molecules moving from the bulk solvent into the crystal, could in principle occur and be monitored, which would allow to quantify the hydration process undergone by the crystal. 
The disorder of the O6 groups in the trajectories of these simulations was analysed in the same way explained in the previous section. In addition, the evolution of the water content of the $\alpha$-CD cavities was also measured with a custom-made PYTHON script. In a given frame, the script first identifies all the $\alpha$-CD molecules by means of their six O-glycosidic atoms (O4 atoms); it then finds the centre of mass of said atoms (which is a computationally-efficient approximation to the real centre of mass of each molecule) and finally creates a virtual sphererepresenting the $\alpha$-CD cavity - with centre at the centre of mass and radius equal to the average distance between the centre and the $\mathrm{O} 4$ atoms (calculated dynamically) minus the Van der Waals radius of an $\mathrm{O}$ atom $(1.5 \AA)$. In the next step, the script loops over all water molecules in the frame and creates a list of the water content of each sphere, by comparing the coordinates of the sphere and the water molecules. The script repeats this process for each frame of the trajectory to output a graph of the evolution of the water content of each $\alpha$-CD during the simulation length. The evolution is calculated as a moving average of the instantaneous water content in each frame. Finally, results for each independent $\alpha$-CD molecule are averaged, similarly to the space average observed in an unit cell obtained by X-ray diffraction.

\subsubsection{Umbrella sampling}

The simulations described in the two previous sections aimed at determining properties of the hydration process which $\alpha$-CD undergoes under pressure from the point of view of geometric parameters - position of O6 groups and water molecules - but do not allow derivation of energy values, mainly due to insufficient sampling of intermediate states between the equilibrium positions. Umbrella sampling is a type of steered MD simulation which overcomes the sampling problem and allows for easy determination of Gibbs free energy curves along known paths (§1.2.3). In the context of this work, umbrella sampling was used to determine the energy curve of the conformational change of O6 groups. 
Three different energy curves of a single O6_1 group were derived from umbrella sampling calculations:

- US1: AP setup at $0.0001 \mathrm{GPa}$

- US2: HP setup at $0.65 \mathrm{GPa}$

- US3: AP setup at $0.65 \mathrm{GPa}$

All setups in this section correspond to those described in \$5.2.4.1; the energy curves correspond to an O6 group deep within the crystal which feels no effects from the bulk solvent. The calculations follow the same principle stated previously: to replicate the behaviour of the corresponding stable form at ambient- and high-pressure conditions (US1 and US2), plus an additional reference calculation to control for model bias (US3).

Each calculation is composed of 120 independent simulations, $1.5 \mathrm{~ns}$ in length each. The rest of the simulation parameters were identical to those in Table 5.2 (frame output was increased to every 5000 steps, producing only 300 frames for visual inspection; generation of energy curves does not rely on frame output). In each simulation the torsion angle $\mathrm{C} 4-\mathrm{C} 5-\mathrm{C} 6-\mathrm{O} 6$ of the $\alpha-\mathrm{CD}$ unit 1 was set to a different value and restrained by a harmonic potential with force constant $2 \mathrm{~kJ} / \mathrm{mol}$, effectively restraining the angle to rotate within $\pm 4^{\circ}$ of its initial position. The angle was set to $180^{\circ}$ in the first simulation, and subsequently moved by $3^{\circ}$ to complete a full $360^{\circ}$ rotation in 120 simulations. Because the degree of rotation allowed by the harmonic potential is $\pm 4^{\circ}$, but the central position is changed only $3^{\circ}$ in every simulation, there is a certain degree of overlap of the sampled space between contiguous simulations, which is necessary to compute reliable energy curves. Stronger potentials are usually not desirable, as they would excessively restrict the flexibility of the torsion angle, limiting the overlap between simulations and forcing to perform more of them, with the consequent increase in computational cost. Weaker potentials tend to be a better choice, as they allow for wider sampling in each simulation, which in turn allows to space out the potentials reducing the number of simulations needed; albeit for this particular system it was found that the torsion 
angle skewed towards certain values if weaker potentials were used. The weighted histogram analysis method, as implemented in wHAM, ${ }^{24}$ was used to derive the energy curves from the simulations.

\subsubsection{Metadynamics}

In the same way that umbrella sampling has been used to estimate the energy change associated with the conformational change of O6_1, metadynamics calculations were implemented to determine the energy cost of incorporating a water molecule into the cavity of an $\alpha$-CD molecule. The main difference with respect to the previous sections is that, while the path followed by the torsion angle on its rotation can be easily described, the path followed by a water molecule during an inclusion event remains unknown. Metadynamics, although very time consuming, provides a way to measure the Gibbs free energy landscape of a complete unit cell, from which the minimal energy path that a molecule can follow can be extracted $(\$ 1.2 .3)$.

A single $400 \mathrm{~ns}$ simulation was performed at $0.65 \mathrm{GPa}$, based on the HP setup described in §5.2.4.1. The rest of the simulation parameters were identical to those in Table 5.2. 3D Gaussian potentials, simply referred as hills $-0.5 \mathrm{~kJ} / \mathrm{mol}$ in height and $0.4 \AA$ in width in all directions were applied to the position of the water molecule $\mathbf{W C}$ of $\alpha$-CD unit 10 (to define the unit cell to be explored in the centre of the simulation box) every 1 ps, forcing the molecule to move continuously across the unit cell. The energy calculation was limited to a single unit cell inside the simulation box by creating soft potential walls (force constant of $200 \mathrm{~kJ} / \mathrm{mol}$ ) $1 \AA$ away of the unit cell faces, which do not allow the target water molecule to cross through them. 1 A-thick soft walls were used to ensure that the target molecule gently bounces back inside the limited region rather suffering an abrupt change in trajectory, which might cause errors when computing energies near the walls. Well-tempered metadynamics was used, that is, the Gaussian hills gradually decreased in size as the simulation progressed. This generates a complete, albeit low resolution, energy landscape in a relatively short time, while the rest of the simulation length is devoted to increase the level of detail to produce more 
accurate energies, as opposed to mapping each area at high resolution before moving to the next one. Well-tempered dynamics offers two main advantages: it is possible to stop the simulation at any time and still obtain results, and it provides a simple way to monitor the convergence of the calculation, by looking at the moving average of the height of the Gaussian hills, which tends to zero (Fig. 5.4).

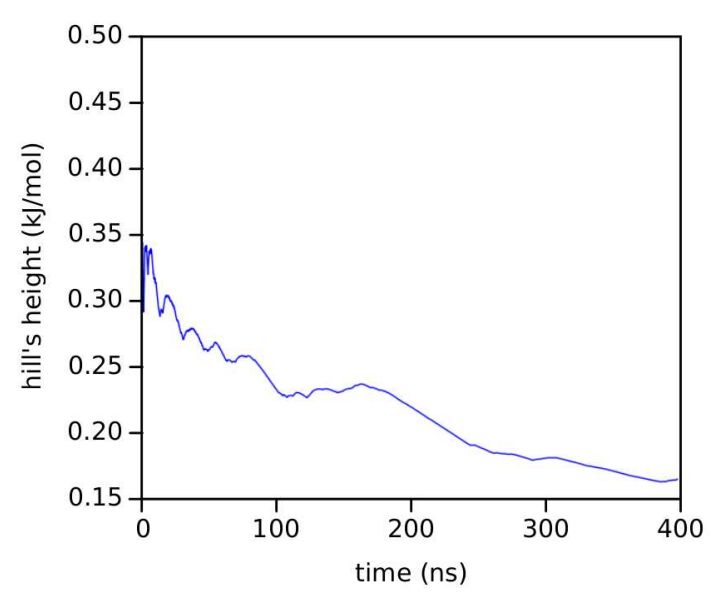

Figure 5.4: Convergence of the metadynamics calculation. Hill's height decreases rapidly at the beginning, halving in $100 \mathrm{~ns}$. Decrease over 300 ns occurs very slowly. Convergence has not been reached after $400 \mathrm{~ns}$; however, qualitative results can be considered accurate at this point.

Free energies are derived from the value of the potential applied in each step and the position in which it is applied, measured as the position of the target molecule relative to its initial position. Therefore, it is exceedingly important to avoid any rotation or drift of the system inside the simulation box. For an infinite crystal, as it is the case here, this can be achieved by fixing the position of a single atom. Fixing atoms can bias the results, and for this reason the fixed atom $\left(\mathrm{C} 1 \_1\right.$ of $\alpha$-CD unit 1) has been chosen outside the unit cell in which the calculation is performed.

NAMD has no implementation to use atomic coordinates as targets of energy calculations. PLUMED (v2.1.1), ${ }^{25}$ a plugin which implements such functionality, was used in combination with NAMD for this simulation. SUM_HILLS, a tool integrated in PLUMED, was used to process the trajectory into a free energy landscape. To represent the 3D energy landscape the sampled space has been divided in to voxels (cubes $0.14 \AA$ in linear length), each of them being characterised by its position within a 3 D grid and energy values. A PYTHON script implementing MAYAVI ${ }^{26}$ has been written to create a coloured $3 \mathrm{D}$ representation of the energy 
landscape from the SUM_HILLS output. The 3D landscape can only be accessed electronically, and for the purpose of $2 \mathrm{D}$ representation the script allows to produce slices of the landscape.

\subsection{Results and discussion}

\subsubsection{Crystallographic evidence for hydration}

Crystallographic results are based on three structures of $\alpha$-CD at three different pressures:

- St1: at 0.0001 GPa (CSD reference code: CHXAMH02 ${ }^{2}$ )

- St2: obtained by direct compression to $0.20 \mathrm{GPa}$ of a crystal grown under ambient-pressure conditions, as described in $\S 5.2 .1$

- St3: obtained by in-situ crystallisation at 0.65 GPa (CSD reference code: REGPAW ${ }^{12}$ )

The three structures were determined from different crystals, hence a compression study was performed to check whether the lattice parameters were consistent across all structures. Lattice parameters of all structures can be found in Table 5.3; Fig. 5.5 shows the results in graphic form. Axes $a$ and $c$ compress under the application of pressure, while axis $b$ elongates; overall, the unit-cell volume decreases. The variation of lattice parameters follows a similar trend for all experiments, with the exception of the $b$ axis, which is longer at $0.55 \mathrm{GPa}$ (from the compression study) than at 0.65 (from the in situ crystallisation experiment). This could be due to the fact that these pressure points were measured with different crystals, or could also be due to an experimental error because of the very low completeness of the $0.55 \mathrm{GPa}$ dataset (31\%). Nevertheless, given that the rest of the parameters follow similar trends, even when coming from different crystals, it is considered that the results coming from different crystals are consistent.

A comparison of the packing of $\mathbf{S t 1}, \mathbf{S t 2}$, and $\mathbf{S t 3}$, using the Crystal Packing Similarity module in MerCury ${ }^{27}$ (using only the core $\alpha$-CD structure, without $\mathrm{O} 2, \mathrm{O} 3, \mathrm{C} 6$, and $\mathrm{O} 6$ atoms) shows that the three structures are very similar. The RMS between St1 and St2 is 0.097, 
5.3. Results and discussion
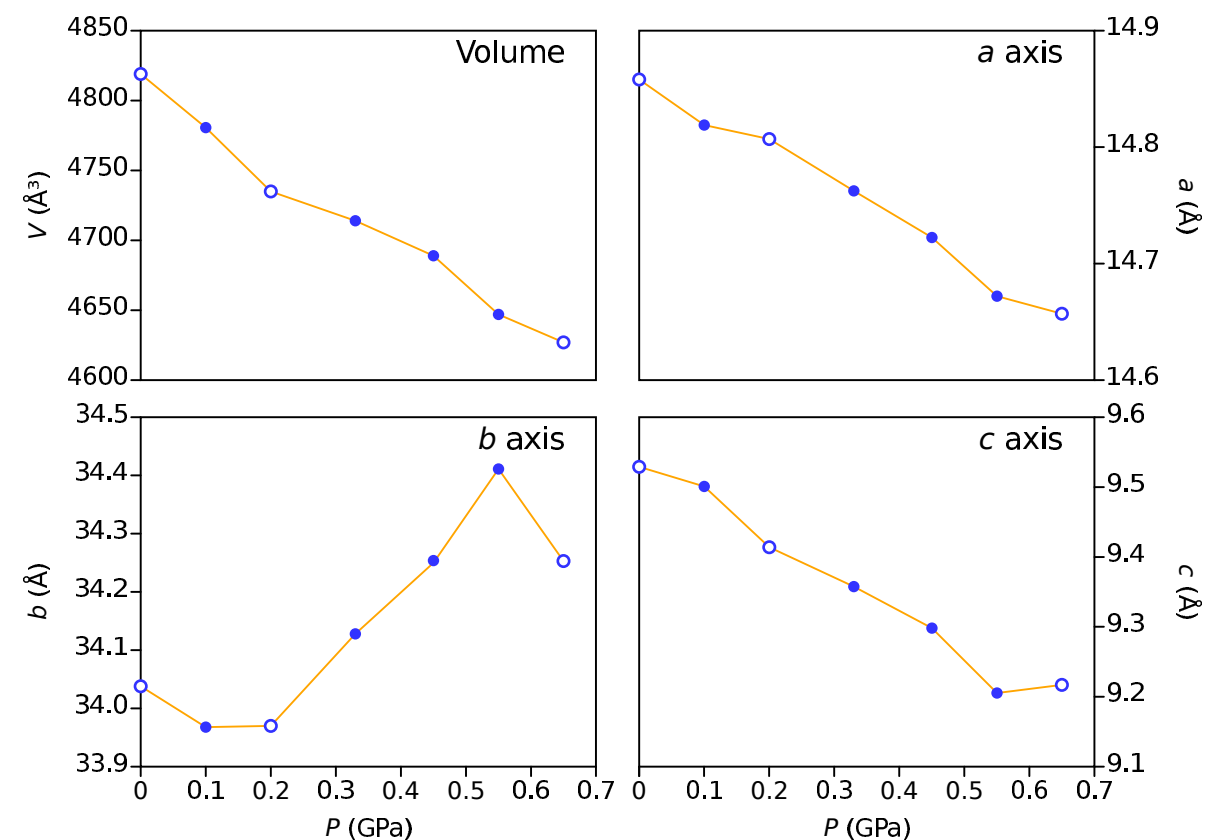

between $\mathbf{S t 1}$ and $\mathbf{S t 3}$ is 0.289, and between $\mathbf{S t 2}$ and $\mathbf{S t 3}$ is 0.196; the small difference can be attributed to the effects of pressure. In any case, the 15 molecules constituting the cluster used in the comparison match across the three structures.

The hydration process undergone by $\alpha$-CD under pressure is characterised by the following (see also Table 5.4 and Fig. 5.6):

- The water content in the cavity of the $\alpha$-CD molecules remains constant at 2 molecules (WA and WB) up to $0.10 \mathrm{GPa}$. Above this pressure, the water content gradually increases up to 2.8 molecules over $0.55 \mathrm{GPa}$. Inclusion of water molecules takes place via two partially-occupied positions (WC and WD), with similar fractional coordinates observed across all structures.

- The water content outside the cavity, 4 water molecules per $\alpha$-CD

\begin{tabular}{lccccc}
\hline Structure & $\boldsymbol{P}(\mathbf{G P a})$ & $\boldsymbol{a}(\AA)$ & $\boldsymbol{b}(\AA)$ & $\boldsymbol{c}(\AA)$ & $\boldsymbol{V}\left(\AA^{\mathbf{3}}\right)$ \\
\hline $\mathbf{S t 1}^{2}$ & 0.0001 & $14.858(4)$ & $34.038(10)$ & $9.529(2)$ & $4819(2)$ \\
$\mathbf{S t 2}^{12}$ & 0.20 & $14.8069(13)$ & $33.970(3)$ & $9.4140(8)$ & $4735(1)$ \\
St3 $^{12}$ & 0.65 & $14.657(5)$ & $34.253(14)$ & $9.217(4)$ & $4627(3)$ \\
\hline \multirow{3}{*}{ Compression $^{*}$} & 0.10 & $14.819(2)$ & $33.968(3)$ & $9.501(1)$ & $4782(2)$ \\
& 0.33 & $14.762(2)$ & $34.128(3)$ & $9.3576(11)$ & $4714(2)$ \\
& 0.45 & $14.722(2)$ & $34.254(4)$ & $9.2982(11)$ & $4689(2)$ \\
& 0.55 & $14.672(3)$ & $34.411(5)$ & $9.2053(16)$ & $4647(2)$ \\
\hline
\end{tabular}

Figure 5.5: Variation of the lattice parameters and unit-cell volume of $\alpha$-CD form I with pressure. Parameters corresponding to structures St1, St2, and St3 are indicated with an empty icon. Parameters corresponding to structures from the compression study, with a full icon. A line linking all icons has been included for visual reference only.
Table 5.3: Lattice parameters of $\alpha$-CD form I at different pressures. 
molecule, remains invariant during compression.

- The group O6_1 can adopt two conformations: $(+)$ and (-), pointing inwards and outwards the cavity, respectively. As the inclusion of water progresses, the $(-)$ conformation becomes increasingly populated, from $10 \%$ at $0.10 \mathrm{GPa}$ to $50 \%$ at $0.55 \mathrm{GPa}$.

- Changes in structure and hydration can occur in the solid state and do not require recrystallisation.

Table 5.4: Summary of the differences observed in $\alpha$-CD form I at different pressures.

\begin{tabular}{|c|c|c|c|c|c|}
\hline \multirow{2}{*}{ Structure } & \multirow{2}{*}{$P(\mathrm{GPa})$} & \multicolumn{2}{|c|}{ Water molecules $^{a}$} & \multicolumn{2}{|c|}{ O6_1 conformation $(\%)$} \\
\hline & & In & Out & $(+)$ & $(-)$ \\
\hline $\mathrm{St1}^{2}$ & 0.0001 & 2 & 4 & 90 & 10 \\
\hline St2 & 0.20 & 2.4 & 4 & 80 & 20 \\
\hline $\mathrm{St3}^{12}$ & 0.65 & 2.8 & 4 & 50 & 50 \\
\hline \multirow{4}{*}{ Compression } & 0.10 & 2 & 4 & 90 & 10 \\
\hline & 0.33 & 2.6 & 4 & 75 & 25 \\
\hline & 0.45 & 2.7 & 4 & 65 & 35 \\
\hline & 0.55 & 2.8 & 4 & 50 & 50 \\
\hline
\end{tabular}

${ }^{a}$ Number of water molecules located inside and outside of the $\alpha$-CD cavity.

Figure 5.6: Increase in the occupancy of WC and WD (blue) and the (-)-gauche conformation of O6_1 (orange) with pressure. Parameters corresponding to structures St1, St2, and St3 are indicated with an empty icon. Parameters corresponding to structures from the compression study, with a full icon. Straight lines are given for visual reference only.

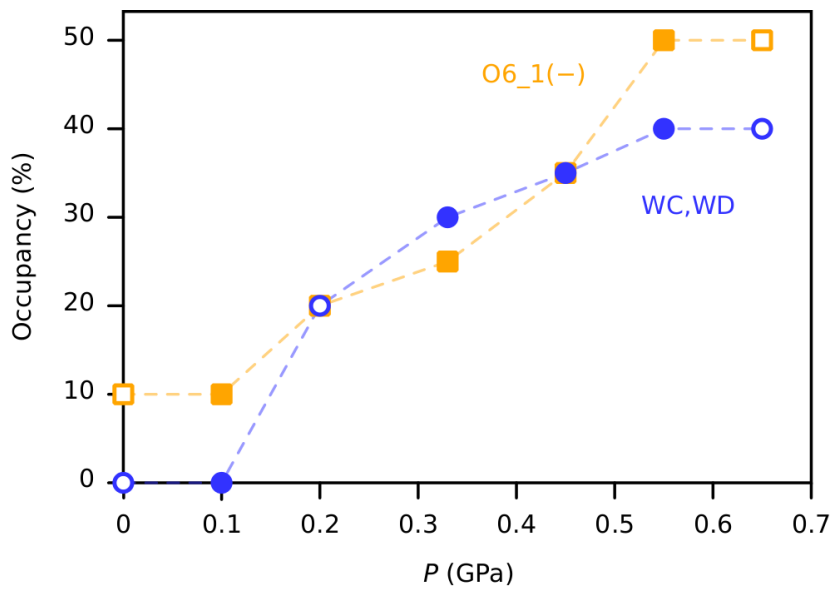

These observations evidence that the inclusion of water in the $\alpha$-CD cavities and the increased disorder of the $06 \_1$ group must be related to the increase of pressure. Previous observations showed that the displacement of the O6_1 group from the (+) to the (-) conformation increases the solvent-accessible volume of the cavity, however, that is not sufficient to explain how the inclusion happens, because there are no channels in the structure which could allow for the movement of water to reach the cavities. ${ }^{12}$ Three different mechanisms can be proposed to explain the hydration in the solid state: 
- Sequential, disorder-driven: the conformational change of O6_1 is caused by pressure, opening the cavity and allowing water to flow in. The opening of the cavities should happen in such a way that channels across the crystal are created.

- Sequential, inclusion-driven: pressure forces the water inside the crystalline structure and the molecules are accommodated in the $\alpha$-CD cavities; the conformation of O6_1 must change in order to allow the flow of water.

- Concerted: pressure causes both effects - water flow inside the crystal and O6_1 conformational change - at the same time, without said effects being related directly.

The likelihood of each mechanism is discussed in detail in the next section based on the results from the MD calculations.

\subsubsection{Hydration mechanism by molecular dynamics}

The validity of the results obtained by MD depends on the performance of the force field at the conditions under which each simulation is carried out, as introduced in $\$ 1.2 .4$. The results outlined in this section are in qualitative agreement with experimental values measured by single-crystal X-ray diffraction; however, a quantitative interpretation would not be appropriate, as numerical results from the simulations tend to be shifted with respect to the experimental observations. For instance, simulated unit-cell volumes can differ up to $\pm 0.5 \AA^{3}$ from the experimental ones and Gibbs free energy minima determined from umbrella sampling are systematically shifted (§5.3.2.3). These quantitative differences are likely to stem from the fact that the CHARMM force field has been used here without any modification, and the force constants probably need to be optimised for each specific pressure. Notwithstanding, these results are consistent in all simulations, as explained in the following sections. Such consistency is an argument in favour of the simulations here presented; although all conclusions are drawn from comparison of simulations performed in a similar fashion and the intention here is not to give absolute results. Differences between simulated and experimental results are discussed when required. 


\subsubsection{Equilibrations of infinite crystals}

The simulations of a crystal of $\alpha$-CD in the absence of bulk solvent allow to determine whether the conformational change undergone by 06_1 is triggered by pressure alone or if it requires the inclusion of additional water. Table 5.5 contains information on the relative occupancy of the (-)-gauche conformation of the $\mathrm{O} 6$ groups (pointing outwards the $\alpha$-CD cavity) determined from the four simulations, that is, two different initial setups at two pressures each, as described in §5.2.4.1.

Table 5.5: Relative occupancy of the (-) conformation of the $\mathrm{O} 6$ groups in the simulation of $\alpha$-CD infinite crystals and in the experimental crystal structures. Values approximated to the nearest integer (Appx. $\mathrm{H}$ for complete results).

\begin{tabular}{ccccc|cc}
\hline Setup & AP & HP & AP & HP & \multicolumn{2}{c}{ Crystal structures } \\
$\boldsymbol{P}(\mathbf{G P a})$ & 0.0001 & 0.65 & 0.65 & 0.0001 & $\mathbf{0 . 0 0 0 1} \mathbf{G P a}$ & $\mathbf{0 . 6 5} \mathbf{G P a}$ \\
\hline $\mathbf{0 6 \_ 1}$ & 0 & 48 & 11 & 45 & 0 & 50 \\
$\mathbf{0 6 \_ 2}$ & 99 & 99 & 95 & 99 & 100 & 100 \\
$\mathbf{0 6 \_ 3}$ & 0 & 2 & 0 & 4 & 0 & 0 \\
$\mathbf{0 6 \_ 4}$ & 99 & 99 & 99 & 99 & 100 & 100 \\
$\mathbf{0 6} \mathbf{5}$ & 95 & 97 & 99 & 98 & 100 & 100 \\
$\mathbf{0 6} \mathbf{6}$ & 99 & 99 & 97 & 99 & 100 & 100 \\
\hline
\end{tabular}

The largest change observed with respect to the initial state is an $11 \%$ increase in the disorder of $\mathrm{O}_{-} 1$ of the AP setup simulated at $0.65 \mathrm{GPa}$. This is considered not significant, as it is known that O6_1 can be disordered between the two gauche conformations up to $10 \%$ even at ambient pressure. ${ }^{2}$ The slight differences observed between the simulations of the same setup at different pressures evidence the absence of model bias. It is clear that the pressure at which the simulations are carried out does not have a direct effect on the disorder of the O6 groups, as these remain in a similar state as in the initial setup. This observation points to the sequential, inclusion-driven mechanism of hydration.

\subsubsection{Equilibrations of molecular clusters in water}

These simulations encountered two major problems at both ambient and high pressure: dissolution and movement of the cluster. Dissolution should be avoided, as it reduces the number of $\alpha$-CD molecules in the cluster which can be analysed at the end of the simulation. Furthermore, because dissolution is not reproducible in different simulations, it makes comparison of the results more difficult. Movements of the crystal, mainly rotation and drift, introduce unnecessary complications in 
the analysis of the trajectories (e.g.: redefinition of the reference frame of the crystal in every frame) and can cause instabilities if two clusters from adjacent simulations boxes get in touch. The latter can be avoided by using a simulation box sufficiently large to contain the full rotation of the cluster, at the cost of increasing the amount of bulk solvent and in turn the computational cost of the simulation. The observation of these two effects corresponds to a physically meaningful processes. It can only be controlled by constraining certain simulation parameters, albeit the number of constrains should be kept to a minimum, otherwise the simulated system might not be representative of a crystal.

In this case it was found appropriate to fix a single atom $\left(\mathrm{C} 1 \_1\right)$ of the eight $\alpha$-CD molecules at the corners of the cluster. This is enough to completely avoid movements of the cluster, and also greatly reduces dissolution, as this tends to happen in the corners, where CD molecules are more exposed to the solvent. As indicated in $§ 5.2 .4 .4$, by fixing a single atom per $\alpha$-CD molecule the flexibility of the macrocycle is not compromised. The use of this type of constraints poses an additional problem: because the volume of the cluster is virtually fixed, applying pressure on the system does not lead to the compression of the cluster; it is essentially treated like a very incompressible material. In any case, because of its very small size, compression of the cluster would be negligible when compared to that of the bulk solvent, for which no constraints were applied during the simulation.

Even after the introduction of the constraints, this set of simulations failed to reproduce experimental results. With respect to the O6 groups, it was observed that all of them underwent conformational change to a certain extent, independently of the pressure. Because the final results are an average of all the groups in the cluster, the analysis was repeated separately for the $\alpha$-CD molecules on the outer layer and the core of the cluster, showing that the conformational change only took place in the outer layer, while molecules of the central core remained unaltered.

When computing the number of water molecules inside the $\alpha$-CD cavities, it was found to be 2 at $0.0001 \mathrm{GPa}$ and 2.3 at $0.65 \mathrm{GPa}$. Expecting similar surface effects, the calculation was also performed sepa- 
rately on the outer and core layers of the cluster; here it was found that, while inclusion of water molecules does take place only at high pressure, it is limited to the surface of the crystal. If these simulations were to be scaled to the size of the crystals measured experimentally, a simple surface effect cannot account for an increase of the hydration number from 6 to 6.8. This set of simulations was discarded, on the basis that it could not reliably reproduce experimental results.

\subsubsection{Umbrella sampling}

Fig. 5.7 shows the three Gibbs free energy curves of O6_1 at different conditions calculated by umbrella sampling. All the curves exhibit three minima, with different relative stabilities. The minimum at $187^{\circ}$ corresponds to the $(+)$-gauche conformation, for which an experimental torsion angle of $170.46^{\circ}$ is measured at $0.0001 \mathrm{GPa}$ and $166.22^{\circ}$ at $0.65 \mathrm{GPa}$. The minimum at $53^{\circ}$ corresponds to the $(-)$ conformation, which exists at $0.65 \mathrm{GPa}$ with a torsion angle of $37.62^{\circ}$. There is an additional minimum at $282^{\circ}$, which due to its higher relative free energy can be considered as an intermediate state. The existence of this state has already been addressed in $§ 5.3 .2 .1$. There is a clear systematic difference of $\sim 15^{\circ}$ between the values determined from the simulation and those measured experimentally. The origin of this shift is currently unknown, however, the curves can be considered qualitatively correct, as discussed at the beginning of this section.

At 0.0001 GPa, from US1 (see §5.2.4.3 for definitions), the preferred conformation of O6_1, with the lowest Gibbs free energy, is (+)-gauche, that is, pointing towards the cavity. Although this is usually not the preferred orientation, it is favoured for this particular glucose ring to stabilise the water inside the $\alpha$-CD cavity by $\mathrm{H}$ bond formation. The (-) conformation is slightly higher in energy, $4.5 \mathrm{~kJ} / \mathrm{mol}$, and can also exist at ambient pressure, as known from the fact that a small degree of disorder in between the two conformations is observed in the crystal structure at $0.0001 \mathrm{GPa}$. Notwithstanding this, the energy barriers for the rotation are high enough to hinder the conformational change, that is, the disorder is static rather than dynamic. At 0.65 GPa, from US2, 


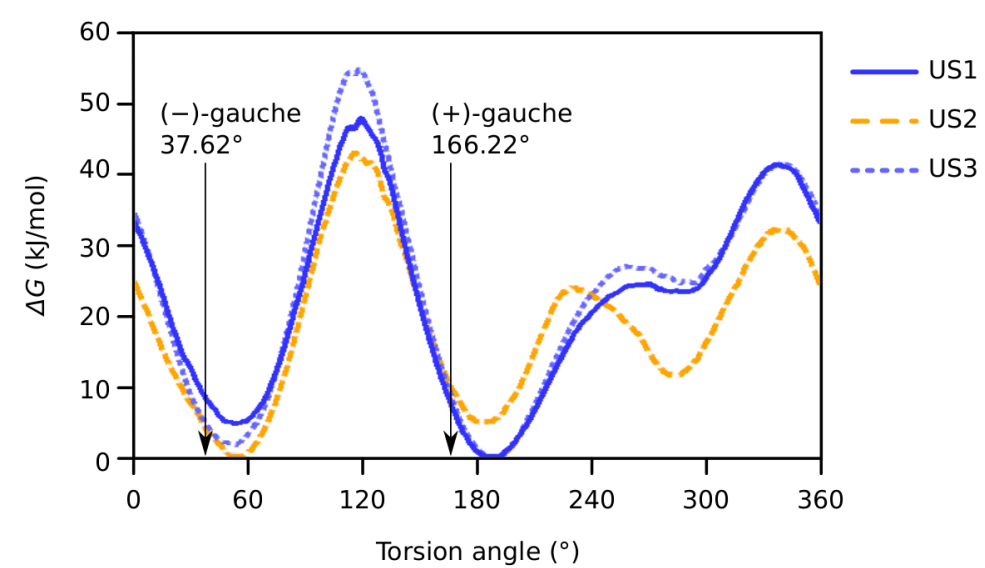

${ }^{a} 0^{\circ}$ corresponds to $\mathrm{C} 4-\mathrm{C} 5$ and $\mathrm{C} 6-\mathrm{O} 6$ bonds being eclipsed. The (-)-gauche conformation (O6 outwards the $\alpha$-CD cavity) corresponds to $\sim 60^{\circ}$. The $(+)$-gauche conformation (outwards) corresponds to $\sim 180^{\circ}$. Corresponding torsion angles in the crystal structure at $0.65 \mathrm{GPa}$ are given as a reference. For further information on how the angles are defined, see Appx. H.

the relative energies of the minima are inverted. The $(-)$ conformation is the absolute minimum at this conditions, with the $(+)$ conformation $4.9 \mathrm{~kJ} / \mathrm{mol}$ higher in energy. In addition, the intermediate state is stabilised by $11.5 \mathrm{~kJ} / \mathrm{mol}$ upon increasing pressure, which provides a route with substantially lower energy barriers (a maximum of $20.1 \mathrm{~kJ} / \mathrm{mol}$ ) through which the conformational change can occur.

The energy curve obtained from US3 coincides with that from US1, which indicates that the change in the energy curve is not caused by the application of pressure, but also by the presence of two additional water molecules, WC and WD, which were only present in the HP setup employed in US2. These observations point to the sequential, inclusiondriven mechanism of hydration, as it seems that the disorder of O6_1 is induced by the presence of water.

\subsubsection{Metadynamics}

The 3D relative Gibbs free energy landscape of the unit cell of $\alpha$-CD form I at $0.65 \mathrm{GPa}$, determined from the HP setup, cannot be shown on print and is only available electronically. A script has been written to explore the data and obtain $2 \mathrm{D}$ slices of the landscape ( $\$ 5.2 .4 .4)$. Fig. 5.8 shows slices parallel to the $b c$ plane at different positions in $a$.
Figure 5.7: Gibbs free energy curves for the rotation of $\mathrm{O} 6$ groups measured by the torsion angle $\quad \mathrm{C} 4-\mathrm{C} 5-\mathrm{C} 6-\mathrm{O} 6{ }^{a}$ US1: solid blue, AP setup at 0.0001 GPa. US2: dashed orange, HP setup at $0.65 \mathrm{GPa}$. US3: dotted light blue, AP setup at $0.65 \mathrm{GPa}$. 
Figure 5.8: Slices of the Gibbs free energy landscape of $\alpha$-CD form I at $0.65 \mathrm{GPa}$. Read from left to right (across the two pages) and top to bottom with increasing values of $a$. All slices are parallel to the $b c$ plane, with $b \in[0, b / 2]$ and $c \in[0, c]$ (origin at the bottom right corner), at every $0.42 \AA$ (three voxels) in $a$. Slices at $a$ below $0.00 \AA$ and over $14.80 \AA$ have been included because the calculation extends outside the unit cell due to the use of soft walls to limit the sampled space. The remaining half of the unit cell (along $b$ ) can be generated by symmetry. The approximate outline of the two $\alpha$-CD molecules present in the region (labelled 1 and 2) have been projected on each slice. Energy levels are colour-coded from 0 to $105 \mathrm{~kJ} / \mathrm{mol}$, being blue the lowest energy and red the highest.

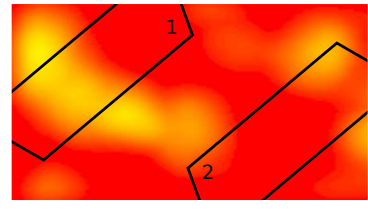

$a=-0.84 \AA$

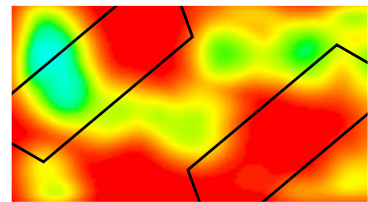

$a=1.68 \AA$

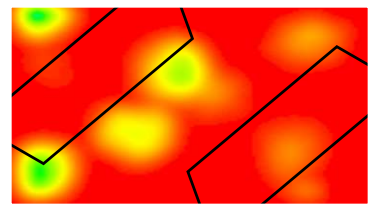

$a=4.20 \AA$

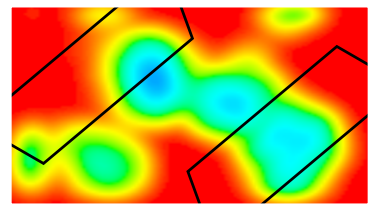

$a=6.73 \AA$

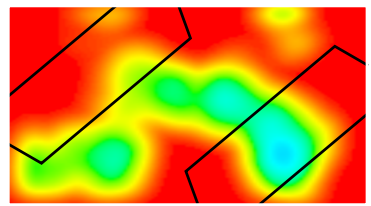

$a=9.25 \AA$

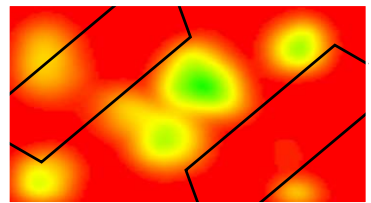

$a=11.77 \AA$

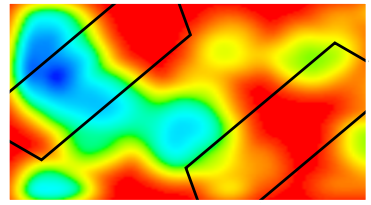

$a=14.30 \AA$

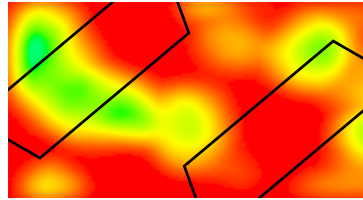

$a=-0.42 \AA$

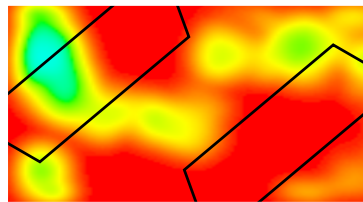

$a=2.10 \AA$

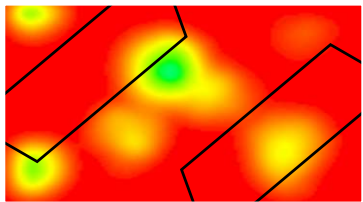

$a=4.63 \AA$

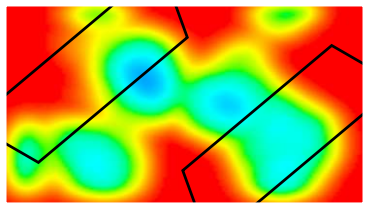

$a=7.15 \AA$

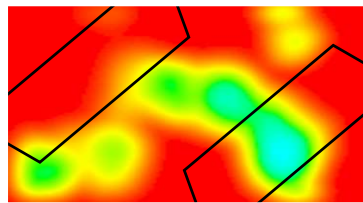

$a=9.67 \AA$

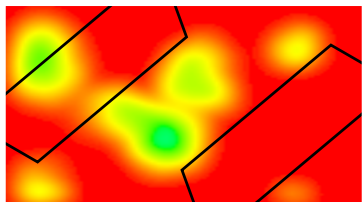

$a=12.19 \AA$

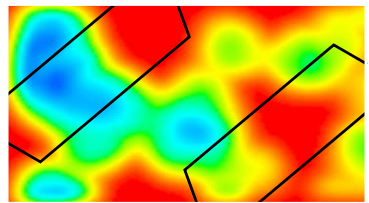

$a=14.72 \AA$

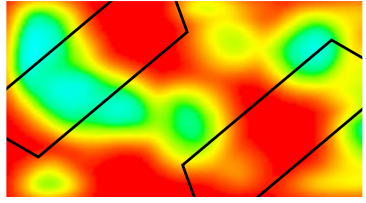

$a=0.00 \AA$

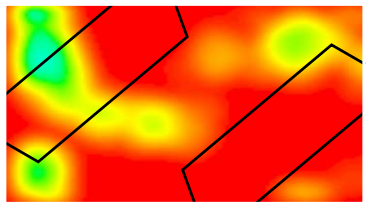

$a=2.52 \AA$

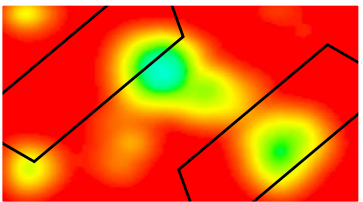

$a=5.05 \AA$

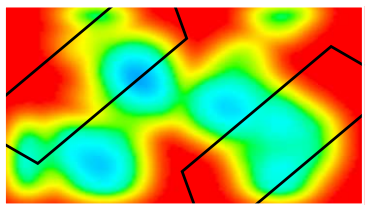

$a=7.57 \AA$

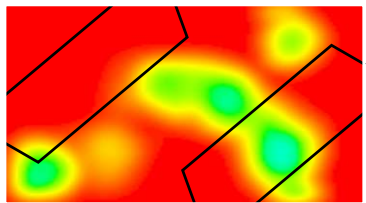

$a=10.09 \AA$

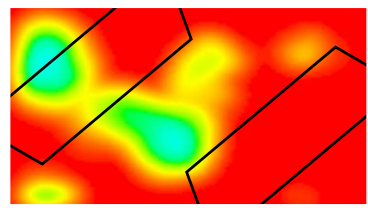

$a=12.61 \AA$

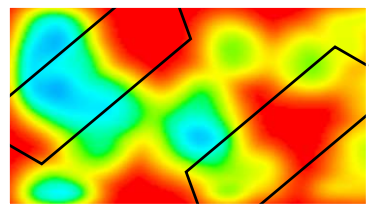

$a=15.14 \AA$ 


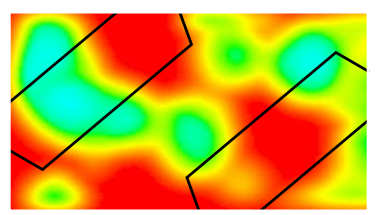

$a=0.42 \AA$

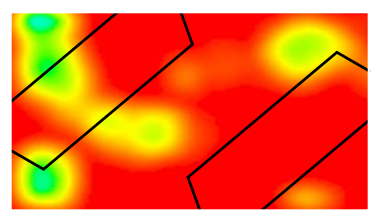

$a=2.94 \AA$

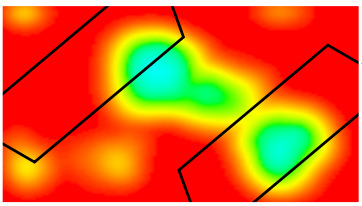

$a=5.47 \AA$

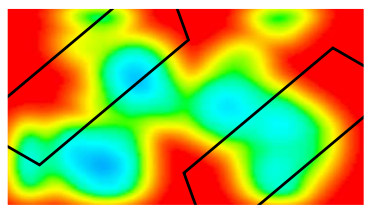

$a=7.99 \AA$

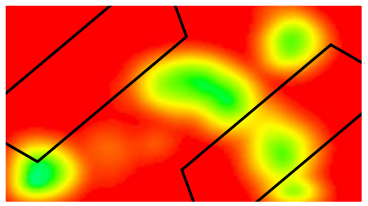

$a=10.51 \AA$

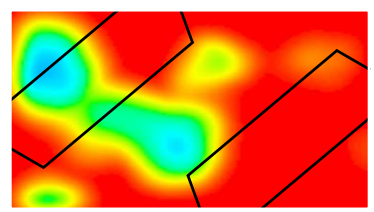

$a=13.03 \AA$

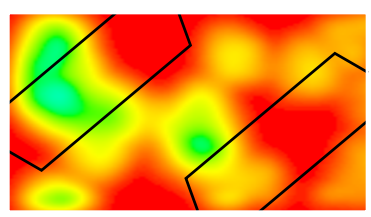

$a=15.56 \AA$

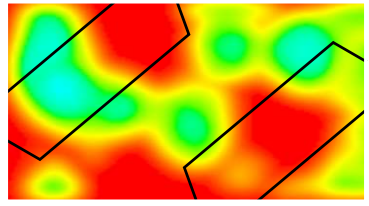

$a=0.84 \AA$

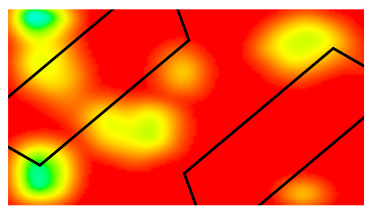

$a=3.36 \AA$

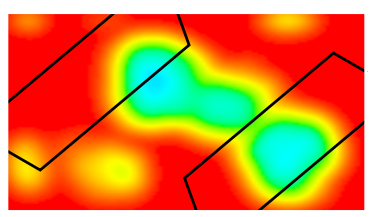

$a=5.89 \AA$

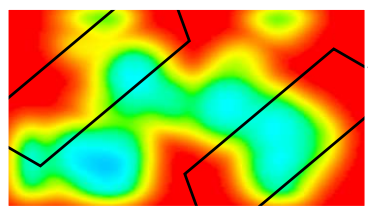

$a=8.41 \AA$

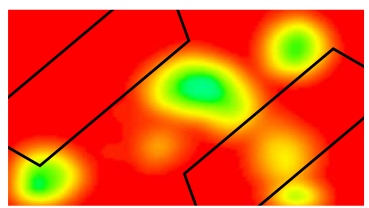

$a=10.93 \AA$

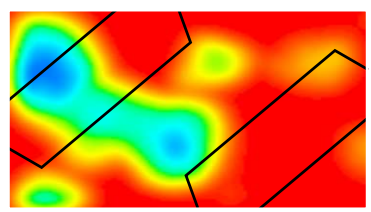

$a=13.45 \AA$

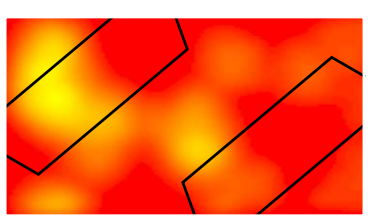

$a=15.98 \AA$

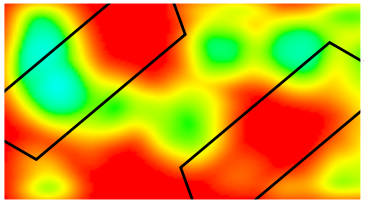

$a=1.26 \AA$

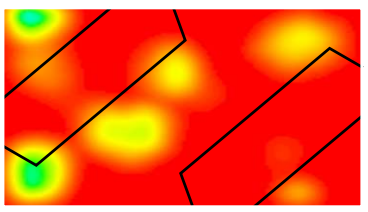

$a=3.78 \AA$

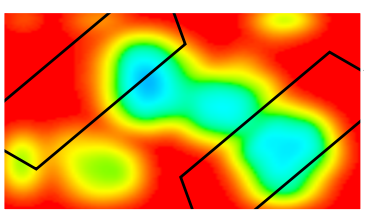

$a=6.31 \AA$

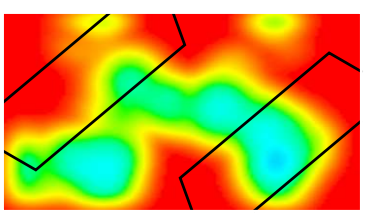

$a=8.83 \AA$

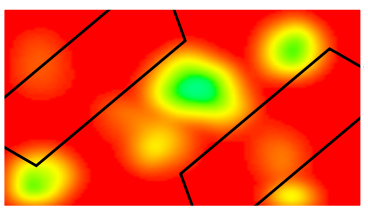

$a=11.35 \AA$

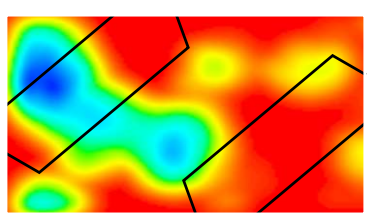

$a=13.88 \AA$

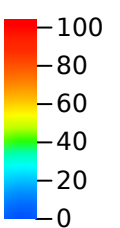

$\Delta \mathrm{G}(\mathrm{kJ} / \mathrm{mol})$ 
The two symmetry-related $\alpha$-CD molecules ( $\mathbf{1}$ and $\mathbf{2}$ in Fig. 5.8) which are almost entirely contained in the space of the unit cell defined in the figure have been also represented.

The energy maps show that the lowest energy regions in the crystal structure, depicted in dark blue, correspond to the interior of the cavities and the intermolecular spaces, were lattice water molecules are located. The highest energies, depicted in red, are associated with regions in which atoms from the $\alpha$-CD molecule cross through the plotted slice. It can be seen between $a=5.47 \AA$ and $a=9.67 \AA$ that there is an area, with energies below $50 \mathrm{~kJ} / \mathrm{mol}$, connecting the cavities of both macrocycles. A water molecule could potentially follow a path across this area to move between the cavities. Further exploration of the geometry of the path, paying special attention to the arrangement of the O6 groups in its vicinity, shows that O6_1 of molecule 1, which exhibits (+)-gauche conformation, effectively blocks the path.

Movement of a water molecule from the intermolecular space into an $\alpha$-CD cavity consists of a destabilising component - breaking the intermolecular interactions involving the water molecule and the group O6_1, which has to rotate to allow for the movement - and an stabilising component-formation of new interactions. Typically, moderate $\mathrm{H}$ bonds, as those seen in the structures of $\alpha$-CD, exhibit energies between 20 and $60 \mathrm{~kJ} / \mathrm{mol},{ }^{28}$ which are in the same order of magnitude as the process being described. For instance, the $P V$ work per $\alpha$-CD at $0.65 \mathrm{GPa}$ is $32.5 \mathrm{~kJ} / \mathrm{mol}$, which could be sufficient to trigger H-bond rearrangement. In the case of the group O6_1, rotation and rearrangement of $\mathrm{H}$ bonds have been estimated at $38.6 \mathrm{~kJ} / \mathrm{mol}$ by umbrella sampling (in two steps, see Fig. 5.7). Other interactions might play a role too, but are likely to contribute in a lesser extent.

\subsubsection{High-pressure hydration mechanism}

Based on the determination of the crystal structure of $\alpha$-CD at three different pressures and the results from MD presented above, it is possible to postulate a sequential, inclusion-driven mechanism for the hydration of an $\alpha$-CD form I crystal induced by high pressure, clearly distinct from 
the induced-fit mechanism which operates at ambient conditions. ${ }^{4}$

In both mechanisms, $\alpha$-CD molecules start in the tense conformation: the overall shape of the macrocycle resembles a distorted circle, with one glucose ring tilted with respect to the others, and two O6 groups in the less-preferred (+)-gauche conformation (Fig. 5.9a). At ambient pressure, the presence of a guest of suitable size causes the conformation to change from tense to round (Fig. 5.9b); in certain cases this allows the inclusion of additional water (Fig. 5.9c). Eventually, the water is displaced in favour of the guest, with the $\alpha$-CD remaining in the round conformation.

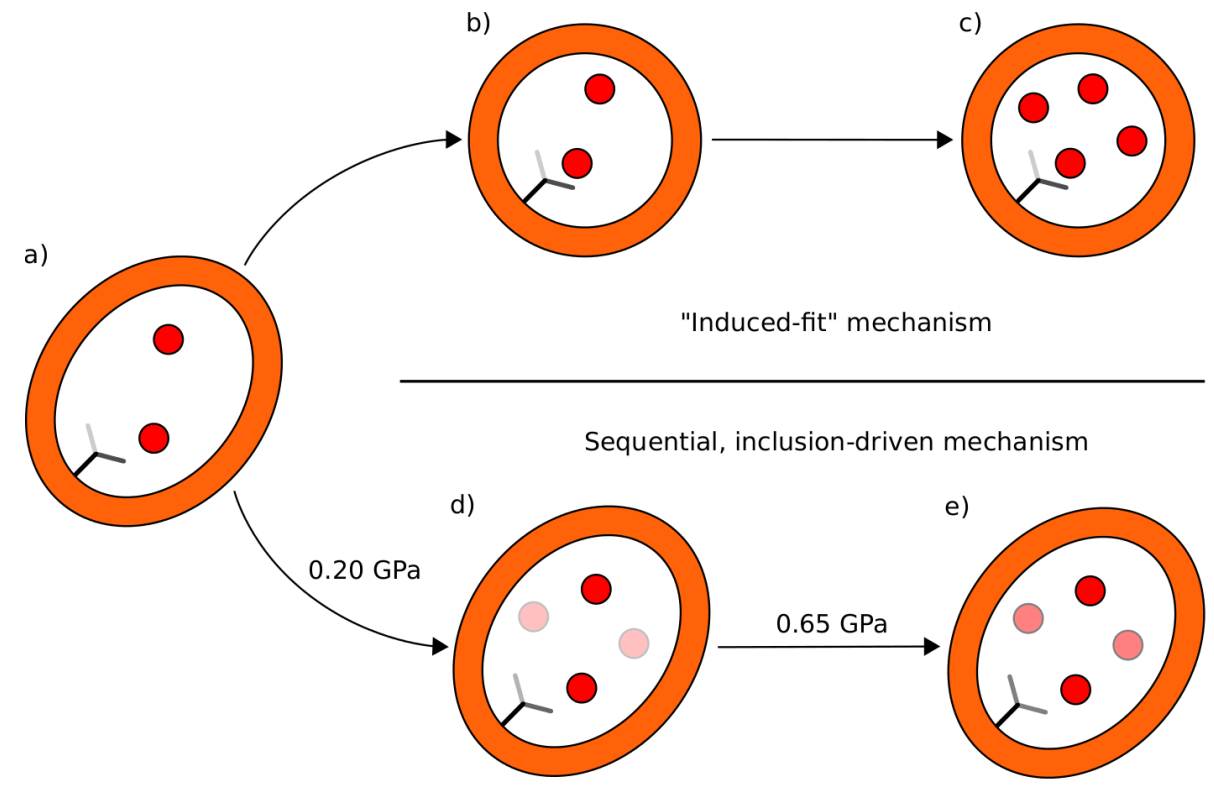

Figure 5.9: Sketch of the two possible inclusion mechanisms operating on $\alpha$-CD form I. a) Initial state, tense conformation. b) $\alpha$-CD changes to the round conformation in the presence of a suitable guest. c) A higher water content in the cavity is possible after the conformational change. d) Upon application of pressure, 0.4 additional water molecules are included in the $\alpha$-CD cavity maintaining the tense conformation, albeit the O6_1 group is partially rotated outwards the cavity. e) 0.8 water molecules are included at $0.65 \mathrm{GPa}$, with O6_1 populating equally

If pressure is applied on form I, bulk water molecules are pushed into the crystal. Overall, water inclusion and crystal volume reduction exboth $(+)$ and $(-)$ conformations.

plain why crystals of $\alpha$-CD dissolve in water upon compression, but given the right conditions (that is, recrystallisation on decreasing pressure) water can be accommodated inside the cavities of the $\alpha$-CD molecules. The process takes place in the solids state, that is, hydration is a continuous process and the structures reported here at different pressures represent different hydration states of the same structure and not different hydrates. In other words, water inclusion is a gradual process that occurs without distorting the overall packing of the crystal structure or 
the conformation of individual $\alpha$-CD molecules, requiring only the conformational change of the O6_1 group, which allows for the movement of water in the absence of natural channels in the crystal and for larger cavity volumes (Fig. 5.9d,e). Energy calculations from MD simulations also support this proposal.

\subsection{Conclusions and outlook}

A series of single-crystal X-ray diffraction experiments on $\alpha$-CD hydrate form I have been undertaken. The crystallographic study shows that a gradual uptake of water occurs upon compression, with the number of water molecules occupying the CD cavity increasing from 2 at ambient pressure to 2.8 at $0.65 \mathrm{GPa}$. Moreover, it clarifies with respect to previous studies that this continuous process takes place in the solid state and does not require in-situ recrystallization. A change in the conformation of one of the hydroxy groups of the macrocycle (O6) accompanies the change in hydration. By means of MD simulations, a feasible mechanism which explains all the features observed in the crystal structures at different pressures has been proposed. Such a mechanism, which is different from the induced-fit mechanism via which $\alpha$-CD forms inclusion complexes in solution, considers that water is first pushed into the crystal upon application of pressure, and then travels through the crystal thanks to temporary opening of channels via a conformational change of the O6 groups.

This study provides an example of the two radically different inclusion mechanisms that take place either in solution or in the solid state. One of these mechanisms is only accessible when pressure is applied to the system, highlighting the importance of exploring this thermodynamic variable for getting the complete picture. In this respect, $\alpha$-CD is an ideal model system to study these phenomena using both diffraction and MD methods. For instance, pressure-induced denaturation of proteins is believed to occur via a similar process - penetration of water molecules into the protein hydrophobic core ${ }^{29,30}$ _although the exact mechanism operating at the molecular level is still not fully understood. 
To the best of our knowledge, combined high-pressure MD and diffraction studies have been performed only on a handful of proteins; this is probably because proteins are by nature considerably challenging to study (both computationally and experimentally); in this respect, focussing on smaller, more amenable model systems, such as CDs, may provide information which could be extrapolated to better understand the behaviour of complex biomolecules.

Enough evidence has been provided to support the new sequential, inclusion-driven hydration mechanism of $\alpha$-CD at high pressure; however, further high-pressure studies would be desirable to fully understand the hydration process, as this can shed light on similar molecular-level processes occurring in other materials. The simulations performed thus far provide indirect evidence in support of one particular mechanism. While the inclusion event does happen during the MD simulations it has not been possible to actually isolate it, probably because it occurs in the time frame between the actual outputs: in order to explore the mechanism long simulations are needed (for instance, to ensure convergence and reproducibility); however, given the enormous amount of data that has been produced, frequent outputs are simply unfeasible. As a next step, shorter simulations focussing on the minimal energy path that water molecules can follow inside the crystal should be performed. This will imply to shift the focus from the cavities to the intermolecular spaces, which have not been considered in this study because their water content does not vary. It is likely that the number of water molecules inside said spaces does in fact change, but their movements cannot be observed by X-ray diffraction. Another aspect to consider in future research is the possible correlation between the high-pressure hydration mechanism and the observation that $\alpha$-CD crystals dissolve in water as pressure is increased. Supposing that the inclusion of water destabilises the crystal, it would be interesting to know if it is possible to include more than 2.8 water molecules per $\alpha$-CD cavity without destroying the crystal. Understanding the dissolution behaviour might help in understanding if and under which conditions high-pressure complexation of small organic molecules might take place in solution. 


\section{References}

[1] P. C. Manor and W. Saenger, J. Am. Chem. Soc., 1974, 96, 36303639 .

[2] B. Klar, B. Hingerty and W. Saenger, Acta Cryst., 1980, B36, 11541165.

[3] W. Saenger and T. Steiner, Acta Cryst., 1998, A54, 798-805.

[4] W. Saenger, M. Noltemeyer, P. C. Manor, B. Hingerty and B. Klar, Bioorg. Chem., 1976, 5, 187-195.

[5] K. K. Chacko and W. Saenger, J. Am. Chem. Soc., 1981, 103, $1708-1715$.

[6] W. Saenger, Nature, 1979, 279, 343-344.

[7] K. Lindner and W. Saenger, Acta Cryst., 1982, B38, 203-210.

[8] R. Puliti, C. A. Mattia and L. Paduano, Carbohydr. Res., 1998, $310,1-8$

[9] D. E. Koshland, The Enzymes, Vol. I, Academic Press, 1959, p. 305.

[10] L. Liu and Q. X. Guo, J. Incl. Phenom. Macrocycl. Chem., 2002, 42, $1-14$.

[11] H. C. Georg, K. Coutinho and S. Canuto, Chem. Phys. Lett., 2005, 413, 16-21.

[12] R. Granero-García, F. J. Lahoz, C. Paulmann, S. Saouane and F. P. A. Fabbiani, CrystEngComm, 2012, 14, 8664-8670.

[13] C. Paulmann, MARTOOLSCP, 2007.

[14] Bruker-AXS, APEX2, Bruker-AXS, Madison, Wisconsin, USA, 2007.

[15] G. M. Sheldrick, Acta Cryst., 2015, C71, 3-8.

[16] C. B. Hübschle, G. M. Sheldrick and B. Dittrich, J. Appl. Cryst., 2011, 44, 1281-1284.

[17] GRADE, http://grade.globalphasing.org.

[18] G. M. Sheldrick, SADABS v2008-1, Bruker-AXS, Madison, Wisconsin, USA, 2008.

[19] O. Guvench, S. N. Greene, G. Kamath, J. Brady, R. M. Venable, R. W. Pastor and A. D. MacKerell, J. Comput. Chem.2, 2008, 29, $2543-2564$ 
[20] O. Guvench, E. Hatcher, R. M. Venable, R. W. Pastor and A. D. MacKerell, J. Chem. Theory Comput., 2009, 5, 2353-2370.

[21] W. L. Jorgensen and J. D. Madura, J. Am. Chem. Soc., 1983, 105, 1407-1413.

[22] J. C. Phillips, R. Braun, W. Wang, J. Gumbart, E. Tajkhorshid, E. Villa, C. Chipot, R. D. Skeel, L. Kalé and K. Schulten, J. Comput. Chem., 2005, 26, 1781-1802.

[23] M. P. Allen, Computational Soft Matter: From Synthetic Polymers to Proteins, Lecture Notes, John von Neumann Institute for Computing, Jülich, 2004, pp. 1-28.

[24] A. Grossfield, WHAM: the weighted histogram analysis method, version 2.0.9, http://membrane.urmc.rochester.edu/content/wham.

[25] G. A. Tribello, M. Bonomi, D. Branduardi, C. Camilloni and G. Bussi, Comp. Phys. Comm., 2014, 185, 604-613.

[26] P. Ramachandran and G. Varoquaux, Comput. Sci. Eng., 2011, 13, $40-51$.

[27] C. F. Macrae, I. J. Bruno, J. A. Chisholm, P. R. Edgington, P. McCabe, E. Pidcock, L. Rodriguez-Monge, R. Taylor, J. van de Streek and P. A. Wood, J. Appl. Cryst., 2008, 41, 466-470.

[28] T. Steiner, Acta Cryst., 2002, E41, 49-76.

[29] M. D. Collins, G. Hummer, M. L. Quillin, B. W. Matthews and S. M. Gruner, Proc. Nat. Acad. Sci. USA, 2005, 102, 16668-16671.

[30] P. Das and S. Matysiak, J. Chem. Phys. B, 2012, 116, 5342-5348. 



\section{High pressure study of the} water-tert-butylamine system

\subsection{Introduction}

Alkylamine hydrates are one of the three types of water inclusion compounds according to Jeffrey's classification. ${ }^{1}$ Although closely related to gas hydrates, there is a major distinction: alkylamine guests are bonded to the H-bonded water network through their amine groups. The existence of these interactions causes the structures of this type of compounds to be more complex than that of the gas hydrates, to the extent that a systematic classification is not possible. Overall, alkylamine hydrates resemble gas hydrates with partially open cages, and are usually referred as semi-clathrates.

Among all alkylamines, ${ }^{t} \mathrm{BA}$ has attracted considerable interest, because its 9.75-hydrate, whose crystal structure was determined in $1967,{ }^{2}$ has long been considered to be the only true clathrate in this family of compounds. The structure of this hydrate is in fact the only known ex- 
ample of a gas hydrate type VI structure. ${ }^{3} \mathrm{~A}$ second hydrate of ${ }^{t} \mathrm{BA}$, a 7.25-hydrate, was reported in $1995 .{ }^{4}$ It exhibits a complex structure formed by a combination of eight different cages, all of them with open edges and faces distorted with respect to regular polyhedra. The crystal structure of six other phases of the water $-{ }^{t} \mathrm{BA}$ system have been recently reported, ${ }^{5}$ showing the existence of three other semi-clathrates (7.75-, 11-, and 17-hydrate), two simple hydrates (with 0.25 and 1 water molecules per ${ }^{t} \mathrm{BA}$ molecule) and the solid form of pure ${ }^{t} \mathrm{BA}$.

A comparison of the densities of all ${ }^{t} \mathrm{BA}$ hydrates shows that a maximum of $1.076 \mathrm{~g} / \mathrm{cm}^{3}$ is expected for a hypothetical 4.32-hydrate. Crystallisation, by means of solvent evaporation or cold gas flow and infrared laser heating techniques, seems to be hindered in the high-density region. In this chapter the crystal structure of two new hydrates are reported, with 5.65 and 5.8 water molecules per ${ }^{t} \mathrm{BA}$ molecule, respectively. Both hydrates were obtained by means of in-situ high-pressure crystallisation. These two new structures provide a further insight into the water $-{ }^{t} \mathrm{BA}$ system and contribute to explain how more compact packing arrangements can be achieved in this type of structures.

In addition, a new anhydrate phase of ${ }^{t} \mathrm{BA}$, characterised by extensive internal disorder, is reported. The low-temperature phase ${ }^{5}$ has been termed here form I, while the newly discovered HP phase is labelled form II. The interactions between ${ }^{t} \mathrm{BA}$ molecules and the disorder in the structure have been studied by MD. Such a study shows that form II is an orientational glass, ${ }^{6,7}$ a structure in which the position of the molecules within the unit cell are related by symmetry, while their orientations are unrelated.

\subsection{Experimental}

\subsubsection{Screening overview}

All samples referred in this chapter were prepared by mixing appropriate volumes of ${ }^{t} \mathrm{BA}$ (Sigma-Aldrich, used as received) with demineralised water to achieve certain molar ratios in $1 \mathrm{~mL}$ of solution. The high volume of solution, especially when compared to the size of the sample chamber 
of a DAC, is justified by the high volatility of ${ }^{t} \mathrm{BA}$. The quantities of ${ }^{t} \mathrm{BA}$ needed to prepare the mixtures poorer in the amine would easily evaporate if they were smaller than the ones used here. For the same reason, all mixtures were prepared and kept cold during the screening, to avoid a change in composition due to the evaporation of ${ }^{t} \mathrm{BA}$.

All the crystallisation experiments were performed in a similar manner. The ${ }^{t} \mathrm{BA}$ :water mixtures were loaded in the DAC and compressed at room temperature until crystallisation occurred. Mixtures richer in ${ }^{t} \mathrm{BA}$ were loaded in the DAC at $263 \mathrm{~K}$, to reduce the volatility of the sample, and brought to room temperature once the DAC was sealed. Subsequently, temperature and pressure cycling were performed to obtain single crystals large enough for diffraction.

In their description of the 9.75-hydrate of ${ }^{t} \mathrm{BA}, \mathrm{McMullan}$ et al. ${ }^{8}$ indicated that crystals were obtained from a 1:8 molar mixture of ${ }^{t} \mathrm{BA}$ :water. The same mixture yielded crystals of a different phase upon compression, based on unit-cell comparison, as crystal quality was not sufficient for a complete structural determination. Mixtures 1:7 and 1:9 were also tried and consistently yielded crystals of the same material, corresponding to a new phase, a 5.8-hydrate, which tends to crystallise in the form of several long thick needles, all stacked in the same direction. Diffraction from such samples occurs in the form of very broad Bragg spots limited to low resolution only, which makes structure determination challenging. Eventually, a suitable single crystal was grown from a 1:7 sample and the structure could be completely determined.

Another new phase, a 5.65-hydrate, was grown from a 1:6 mixture. The last mixture to be tested was 1:3; however, none of the crystals obtained were suitable for X-ray diffraction due to their small size. Over a certain pressure, single crystals stopped growing and instead new crystallisation occurred in the form of thin needles. Unfortunately, no single crystal could be grown from this material and the identity of this phase remains unknown.

The following sections contain further information on each of the crystals which were measured. 


\subsection{5-Hydrate}

\subsubsection{Crystal growth}

$450 \mu \mathrm{m}$

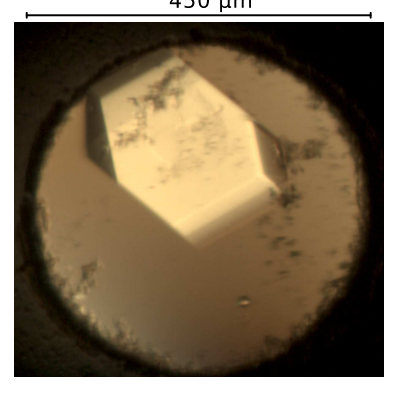

Figure 6.1: Single crystal of ${ }^{t} \mathrm{BA} 5.65$-hydrate at $0.76 \mathrm{GPa}$.

A 1:6 mixture of ${ }^{t}$ BA:water was loaded in a triangular DAC (Appx. I.2) equipped with an Inconel 718 gasket pre-indented to $160 \mu \mathrm{m}$ thickness with a $450 \mu \mathrm{m}$ diameter hole. Crystallisation on direct compression was observed at $0.65 \mathrm{GPa}$. At this pressure crystals melt very fast and grow back very slowly, hence temperature cycling is not appropriate. Pressure was increased to $0.76 \mathrm{GPa}$ to decrease dissolution speed, and a single crystal was obtained by mild temperature cycling in the range $330-350 \mathrm{~K}$ (Fig. 6.1).

\subsubsection{X-ray data collection and processing}

X-ray data were collected on said crystal at 0.76 GPa on a Bruker ApexII CCD diffractometer equipped with a Mo microsource $(\lambda=0.71073 \AA)$. The data collection strategy consisted of $\omega$ and $\phi 0.30^{\circ}$ scans with the DAC in three different orientations (rotations of $120^{\circ}$ around the beam axis). Exposure time was $40 \mathrm{~s}$. Data were integrated following the strategy outlined in §1.1.3.

\subsubsection{Structure refinement}

The structure was solved using the charge flipping method implemented in SUPERFLIP ${ }^{9}$ and subsequently refined by full-matrix least squares against $F^{2}$ using SHelXL v2014/7, ${ }^{10}$ through the GUI ShelXle. ${ }^{11}$ For the ${ }^{t} \mathrm{BA}$ molecules, non-H atoms were refined anisotropically with their ADPs subject to similarity restraints, unless very disordered, in which case isotropic refinement was preferred. Restraints on bond lengths and angles were used to ensure proper geometry. $\mathrm{H}$ atoms of methyl groups were placed in idealised positions and refined riding on their parent atoms. For methyl groups lying on special positions, $\mathrm{H}$ atoms were placed similarly, but their coordinates were later constrained to the special position when needed and spare symmetry equivalents were removed; positions were no longer refined but kept riding on the corresponding carbon atom. $\mathrm{H}$ atoms were placed only in one of the symmetry-independent 
amine groups; positions were located through electron density maps and then kept riding on the nitrogen atom. The rest of the amine groups were disordered and $\mathrm{H}$ atoms could not be placed. In water molecules, all oxygen atoms were located through Fourier difference maps and refined anisotropically, unless residing on special positions. Each oxygen atom was assigned four half-occupied $\mathrm{H}$ atoms in the direction of the vertices of a tetrahedron, which coincide with the direction of the $\mathrm{H}$ bonds in the structure. The positions of the $\mathrm{H}$ atoms were refined constrained to move along with the oxygen atoms they are attached to. A final $R$ factor of $6.54 \%$ was obtained. For further crystallographic data see Table 6.1.

\subsubsection{8-Hydrate}

\subsubsection{Crystal growth}

A crystal was grown by loading a sample of the 1:7 mixture ${ }^{t} \mathrm{BA}$ :water in a DAC (Appx. I.2) equipped with a $200 \mu \mathrm{m}$ gasket pre-indented to a thickness of $160 \mu \mathrm{m}$ and a sample chamber $300 \mu \mathrm{m}$ in diameter. Upon fast compression, crystallisation occurred at $0.80 \mathrm{GPa}$ in the form of fine needles (Fig. 6.2a). Such material melts easily during temperature cycling, at the same time that single crystals start to form (Fig. 6.2b). Finally, two single crystals were obtained inside the sample chamber at $0.61 \mathrm{GPa}$ (Fig. 6.2c).

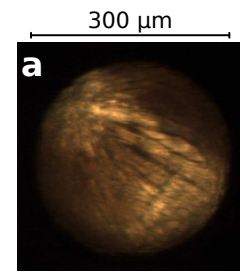

$0.80 \mathrm{GPa}$

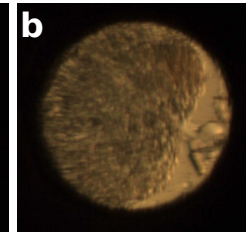

$0.87 \mathrm{GPa}$

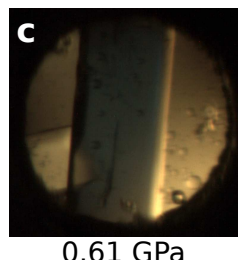

$0.61 \mathrm{GPa}$
Figure 6.2: Crystals of ${ }^{t}$ BA 5.8-hydrate. a) Thin needles obtained by fast compression. b) Needles and small single crystals coexisiting at $0.87 \mathrm{GPa}$ after temperature cycling. c) Two single crystals at $0.61 \mathrm{GPa}$.

\subsubsection{X-ray data collection and processing}

Data were collected on a MAR345 image plate using an INCOATEC microfocus $(\mathrm{I} \mu \mathrm{S})$ source of Ag radiation (0.56085 $\AA$ ). Data collection strategy consisted of $0.5^{\circ} \phi$ scans at three different orientations of the DAC (rotations of 90 and $120^{\circ}$ around the beam axis). Frames collected in this 
detector were converted from MAR to SFRM format by using the tools included in ApEx2. ${ }^{12}$ Data were integrated following the strategy outlined in $\S 1.1 .3$.

Reflections originating from the two crystals in the sample chamber could be indexed in the frames. The best integration procedure consisted in indexing both crystals and integrating according to the two possible lattices. Subsequently, TwinABs ${ }^{13}$ was used to apply absorption correction and to extract only the reflections corresponding to the largest crystal to proceed with the refinement of the structure. Each run was processed individually and all data was finally merged with SORTAV. ${ }^{14}$

\subsubsection{Structure refinement}

The structure was solved using SUPERFLIP ${ }^{9}$ and subsequently refined by full-matrix least squares against $F^{2}$ using SHELXL v2014/7, ${ }^{10}$ through the GUI ShelXle. ${ }^{11}$ For ${ }^{t}$ BA molecules, non-H atoms were refined isotropically. Restraints on bond lengths and angles were used to ensure proper geometry. $\mathrm{H}$ atoms of methyl groups were placed in idealised positions and refined riding on their parent atom. Each nitrogen atom was assigned three partially-occupied $\mathrm{H}$ atoms in the directions of the vertices of a tetrahedron, which coincide with the directions of the $\mathrm{H}$ bonds in the structure. The positions of the $\mathrm{H}$ atoms were refined at first subject to restraints and then constrained to move along with the nitrogen atoms they are attached to, in order to reduce the number of parameters. For water molecules, all oxygen atoms were located through Fourier difference maps and refined anisotropically. Each oxygen atom was assigned four half-occupied $\mathrm{H}$ atoms in a similar manner than for the amine groups. A final $R$ factor of $10.49 \%$ was obtained. For further crystallographic data see Table 6.1.

\subsubsection{Pure tert-butylamine form II}

\subsubsection{Crystal growth}

Pure ${ }^{t} \mathrm{BA}$ was loaded in a square DAC (Appx. I.1) equipped with a steel gasket $230 \mu \mathrm{m}$ thick and a sample chamber $280 \mu \mathrm{m}$ in diameter. Loading 
6.2. Experimental

\begin{tabular}{|c|c|c|}
\hline & 5.65-hydrate & 5.8-hydrate \\
\hline \multicolumn{3}{|l|}{ Crystal data } \\
\hline Chemical formula & $\mathrm{C}_{4} \mathrm{H}_{11} \mathrm{~N} \cdot 5.65 \mathrm{H}_{2} \mathrm{O}$ & $\mathrm{C}_{4} \mathrm{H}_{11} \mathrm{~N} \cdot 5.8 \mathrm{H}_{2} \mathrm{O}$ \\
\hline Molecular mass ( $\mathrm{g} \mathrm{mol}^{-1}$ ) & 174.92 & 177.63 \\
\hline Temperature (K) & $298(2)$ & $298(2)$ \\
\hline Pressure (GPa) & $0.76(5)$ & $0.61(5)$ \\
\hline Crystal system & orthorhombic & monoclinic \\
\hline Space group & Cmca & $P 2_{1} / n$ \\
\hline$a(\AA)$ & $17.2082(6)$ & $12.5091(13)$ \\
\hline$b(\AA)$ & $15.6395(6)$ & $22.213(3)$ \\
\hline$c(\AA)$ & $30.9066(8)$ & $18.766(2)$ \\
\hline$\beta\left(^{\circ}\right)$ & & $92.985(3)$ \\
\hline$V\left(\AA^{3}\right)$ & $8317.8(5)$ & $5207.1(10)$ \\
\hline$Z$ & 32 & 20 \\
\hline Crystal size (mm) & $\sim 0.25 \times 0.20 \times 0.10$ & $\sim 0.30 \times 0.20 \times 0.10$ \\
\hline \multicolumn{3}{|l|}{ Data collection } \\
\hline$\overline{\text { Radiation wavelength }(\AA)}$ & 0.71073 & 0.56085 \\
\hline Absorption correction & SADABS $^{15}$ & TWINABS $^{13}$ \\
\hline$T_{\min }, T_{\max }$ & $0.7439,0.6459$ & $0.7447,0.9330$ \\
\hline \multicolumn{3}{|l|}{ Number of $[I>2 \sigma(I)]$ reflections: } \\
\hline - measured & 15326 & 7547 \\
\hline - independent & 1532 & 3189 \\
\hline - observed & 1101 & 2071 \\
\hline Completeness (\%) & 91.3 & 79.4 \\
\hline$(\sin \theta / \lambda)_{\max }\left(\AA^{-1}\right)$ & 0.30 & 0.38 \\
\hline$R_{\text {int }}(\%)$ & 3.78 & 7.21 \\
\hline \multicolumn{3}{|l|}{ Refinement } \\
\hline$R\left[F^{2}>2 \sigma\left(F^{2}\right)\right](\%)$ & 6.54 & 10.49 \\
\hline$w R\left(F^{2}\right)(\%)$ & 20.06 & 20.86 \\
\hline$S$ & 1.131 & 1.111 \\
\hline Number of reflections & 1532 & 3189 \\
\hline Number of parameters & 208 & 386 \\
\hline Number of restraints & 31 & 70 \\
\hline$\Delta \rho_{\min }, \Delta \rho_{\max }\left(\mathrm{e} \AA^{-3}\right)$ & $-0.244,0.320$ & $-0.229,0.370$ \\
\hline
\end{tabular}

Table 6.1: Crystallographic data and refinement parameters of ${ }^{t} \mathrm{BA}$ and 5.8-hydrate.

174.92

orthorhombic

Cmca

$15.6395(6)$

$8317.8(5)$

32

$\sim 0.25 \times 0.20 \times 0.10$

Crystal size (mm)

Radiation wavelength $(\AA)$

Absorption correction

SADABS $^{15}$

TWINABS ${ }^{13}$

Number of $[I>2 \sigma(I)]$ reflections:

- measured

1532

91.3

0.30

$-0.229,0.370$

was carried out at $263 \mathrm{~K}$ to avoid evaporation of the sample. Solidification in the form of poly-crystalline material occurs at pressures above $0.60 \mathrm{GPa}$ (Fig. 6.3a). This new phase (named form II) is very responsive to changes in pressure and temperature, making the usual annealing procedure for crystallisation challenging. Eventually, a single crystal, occupying the entire sample chamber (Fig. 6.3b), was grown at $0.78 \mathrm{GPa}$. A second smaller crystal grew included into the first one, but could be neglected due to its limited scattering power. Single-crystalline growth of this phase is favoured at temperatures between 310 and $320 \mathrm{~K}$. At lower temperatures the growth is too fast and always results in poly-crystalline material; at higher temperatures the sample melts. 
Figure 6.3: Crystals of pure ${ }^{t}$ BA. a) Polycrystalline material at 0.63 GPa. b) Single crystals. Crystal $\mathbf{I}$ is colourless and occupies the entire sample chamber; crystal II is a small inclusion. c) Compressed single crystal at $1.07 \mathrm{GPa}$.

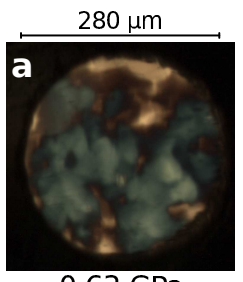

$0.63 \mathrm{GPa}$

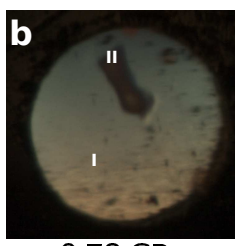

$0.78 \mathrm{GPa}$

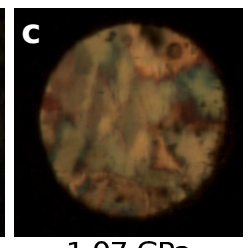

$1.07 \mathrm{GPa}$

X-ray diffraction data showed that this $\mathrm{HP}$ phase of ${ }^{t} \mathrm{BA}$ is very disordered at the molecular level. In an attempt to induce order in the structure, pressure was slowly increased up to $1.07 \mathrm{GPa}$, but the crystal always transitions to a non-diffracting state, as confirmed by X-ray diffraction (Fig. 6.3c). Isochoric thermal annealing under these conditions is not possible, because at such pressures the crystal does not melt (tested at temperatures up to $400 \mathrm{~K}$ ).

\subsubsection{2 $\mathrm{X}$-ray data collection and processing}

Data were collected following the strategy in $\S 6.2 .2 .2$, although with the DAC in a single orientation.

\subsubsection{Structure refinement}

Full-matrix least-squares refinement against $F^{2}$ was performed using SHELXL V2014 $/ 7,{ }^{10}$ through the GUI SHELXLE, ${ }^{11}$ on a solution obtained using SHELXT. ${ }^{16}$ The structure, belonging to space group $P 2_{1} / n$, is characterised by a high degree of disorder. The asymmetric unit contains a single molecule of ${ }^{t} \mathrm{BA}$ disordered over four possible orientations; the central carbon atom occupies a fixed position, but it is not possible to discern which one of the four vertices of the tetrahedral molecule is the nitrogen atom. This has been modelled by setting the likelihood of each terminal atom to be a carbon atom at $75 \%$ and to be a nitrogen atom at $25 \%$. $\mathrm{H}$ atoms were placed in idealised positions for the methyl groups and refined with the riding model. The $75 \%$ occupancy of the carbon atoms was applied to the $\mathrm{H}$ atoms too. Because nitrogen atoms lay in the exact same positions than carbon atoms, no $\mathrm{H}$ atoms were placed on them. Final $R$ factor was $11.73 \%$. For further crystallographic information see Table 6.4. 
All atoms were refined anisotropically; although the ADPs of the terminal atoms are over two times larger than that of the central atom, compared as equivalent isotropic displacement parameters. Such elongation, which occurs mainly in the directions normal to the covalent bonds, could be attributed to a certain degree of libration of the molecule, as if describing a sphere. Different options have been considered to model the libration as rotational disorder. In SHELXL, two partially occupied ${ }^{t} \mathrm{BA}$ molecules instead of one, both sharing the same central carbon atom, have been implemented. CRYSTALS ${ }^{17}$ offers the option of modelling electron density with more complex figures than ellipsoids; ${ }^{18}$ a sphere containing the four terminal atoms was implemented. In both cases there was an increase in the $R$ factor, hence the initial model was maintained, which indicates that full rotational disorder occurs only to a small extent, if at all.

\subsubsection{Molecular dynamics}

MD simulations on ${ }^{t} \mathrm{BA}$ form II have been performed to better understand the disorder and internal structure of this HP phase. For the purpose of the simulations, the disorder has been removed from the refined model, by making one of the four terminal atoms (position 2) a fully occupied nitrogen atom, and the other three atoms fully occupied carbon atoms. Methyl $\mathrm{H}$ atoms were replaced by a rigid methyl group, which is computationally less expensive, given that the structure is likely to be more influenced by the entire methyl group rather than by the individual atoms. Amine $\mathrm{H}$ atoms were geometrically placed using MERCURY, ${ }^{19}$ with bond distances elongated to neutron values, and included in the force field as individual species. The initial setup for the simulations consisted of an arrangement of 24 unit cells (four repetitions along $a$, two along $b$ and three along $c$ ), adding up to $96{ }^{t} \mathrm{BA}$ molecules. This arrangement constitutes the simulation box, which is subsequently put in $\mathrm{PBC}$ to simulate an infinite crystal and energy minimised prior to each simulation.

All simulations here described were performed in a similar manner using GROMACS (v5.0.3). ${ }^{20}$ At first, four different simulations where car- 
ried out to compare the performance of two different force fields with pressure. Each simulation was 2.5 ns in length, consisting of 2 fs steps, and was repeated 5 times, starting always from the same setup, to ensure complete sampling. The NPT ensemble was used in all cases, with the simulations performed at either 0.0001 or $0.78 \mathrm{GPa}$, the latter being the pressure at which form II was crystallised. Two force fields were employed: GROMOS with parameter set $54 \mathrm{~A} 7^{21}$ and OPLS-aa (Optimised Potentials for Liquid Simulations). ${ }^{22}$ The former is the default option in GROMACS, while the latter was specifically designed to reproduce the behaviour of organic liquids, hence it was expected to better suit the needs of this study. Two simulations were performed at $0.0001 \mathrm{GPa}$ using the two different force fields, and two more, also with different force fields, at $0.78 \mathrm{GPa}$.

As suggested by Nemkevich et al., ${ }^{23}$ force-field parameters must ensure that the properties derived from simulations agree with properties measured experimentally, which does not necessarily mean that force-field parameters themselves must agree with experimental parameters. Following this idea, the compressibility of ${ }^{t} \mathrm{BA}$ has been derived from appropriate simulations, to ensure consistency with the force field. Two NVT simulations were performed, one using the initial setup described previously, and another one in which the volume of the simulation box of said setup was enlarged by $1 \%$. The compressibility $(\beta)$ was calculated by measuring the changes in pressure in both simulations (Eq. 6.1). A compressibility of $0.21562 \mathrm{GPa}^{-1}$ was determined in this

$\beta=-\frac{1}{V_{0}} \frac{d V}{d P} \sim$ $-\frac{1}{V_{0}} \frac{\Delta V}{\Delta P}$ way. Further details about the simulation can be found in Table 6.2.

(6.1) After the first tests, the opLS force field was considered to better reproduce the properties of the system than GROMOs, although a re-parametrisation was needed for further improvement. According to Bernardes and Joseph, ${ }^{24}$ it is usually better to use quantum-mechanical charges to derive structural information from a simulation, while the default charges in OPLS are better suited for energy calculations. Although the final aim of these simulations is to obtain FESs, this is done here by measuring structural parameters, hence quantum-mechanical charges were preferred. Charges for each atom of the ${ }^{t} \mathrm{BA}$ molecule were 
6.2. Experimental

\begin{tabular}{ll}
\hline Simulation length & $2.5 \mathrm{~ns}$ \\
Time step & $2 \mathrm{fs}$ \\
Frame output & every 1000 steps $(2 \mathrm{ps})$ \\
Number of frames & 1250 \\
Minimisation & forces below $10.0 \mathrm{~kJ} / \mathrm{mol} / \mathrm{nm}$ \\
Force field & GROMOs $54 \mathrm{~A} 7$ and OPLS-aa \\
PBC & yes \\
Ensemble & $N P T$ \\
Temperature control & Nosé-Hoover \\
Temperature & $298 \mathrm{~K}$ \\
Pressure control & Parrinello-Rahman (isotropic) \\
Pressure & 0.0001 or $0.78 \mathrm{GPa}$ \\
Treatment of interactions & \\
- Covalent & $<9 \AA$ \\
- Coulomb and Van der Waals & $>9 \AA$ (PME) \\
Interactions cutoff & $10 \AA$ \\
Treatment of bonds with H atoms & rigid \\
\hline
\end{tabular}

Table 6.2: Details of the simulations of pure ${ }^{t} \mathrm{BA}$.

computed from a MP2/TZvP calculation in Gaussian03. ${ }^{25}$ Charges of $\mathrm{H}$ atoms belonging to the same type of functional group were averaged; then charges within the same methyl group were added together to make a single entity, as explained previously. Final charges used in the production runs are detailed in Table 6.3. Other parameters of the force field, which mainly control Van der Waals interactions, were left untouched. The reason behind this is that intermolecular interactions in MD simulations, which greatly affect the trajectory, are mainly governed by electrostatic forces, while the main role of Van der Waals interactions is to avoid the molecules clashing.

Pressure coupling was changed from isotropic to anisotropic, to allow for each dimension of the simulation box to compress independently. This function may fail to reproduce unit-cell volumes at high pressures, but it was a sound option in this particular case, based on the simplicity and homogeneity of the system, to allow for more realistic simulations (see $\S 5.3 .2$ for further discussion).

After implementing all these changes, the simulations at 0.0001 and $0.78 \mathrm{GPa}$ were repeated. Two other simulations, at 0.40 and $1.20 \mathrm{GPa}$, were are also performed, to further explore the behaviour of the system.

A Python script was written to analyse the trajectories and produce FESs. The orientation of the ${ }^{t} \mathrm{BA}$ molecules was chosen as an order parameter, in terms of which the trajectories could be expressed. The orientation of each ${ }^{t} \mathrm{BA}$ molecule in each frame is described by means

Table 6.3: MP2/TZVP charges calculated for each atom or group atoms of the ${ }^{t} \mathrm{BA}$ molecule. GROMOS and OPLS charges are given for reference.

\begin{tabular}{lr}
\hline \multicolumn{2}{l}{$\mathrm{MP} 2 /$ TZVP } \\
\hline $\mathrm{C}_{\text {central }}$ & 0.72710 \\
$\mathrm{CH}_{3}$ & -0.13957 \\
$\mathrm{~N}$ & -1.03307 \\
$\mathrm{H}_{\text {amine }}$ & 0.36234 \\
\hline GROMOS & \\
\hline $\mathrm{C}_{\text {central }}$ & 0.00000 \\
$\mathrm{CH}_{3}$ & 0.00000 \\
$\mathrm{~N}$ & -0.83000 \\
$\mathrm{H}_{\text {amine }}$ & 0.41500 \\
\hline OPLS & \\
\hline $\mathrm{C}_{\text {central }}$ & 0.18000 \\
$\mathrm{CH}_{3}$ & 0.00000 \\
$\mathrm{~N}$ & -0.90000 \\
$\mathrm{H}_{\text {amine }}$ & 0.36000 \\
\hline
\end{tabular}




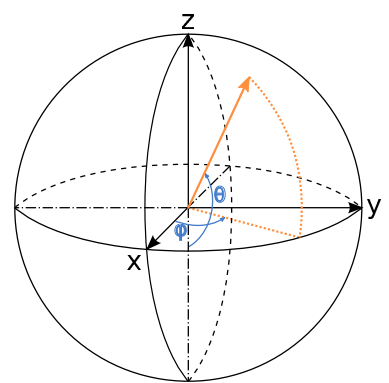

Figure 6.4: Graphic relationship between polar and Cartesian coordinates. $x, y$, and $z$ are the orthonormal axes corresponding to the monoclinic $a, b$, and $c$ axes.

$f_{\text {corrected }}=f \sin (\theta) / 2$

(6.2)

of the vector between the central carbon atom and the nitrogen atom (here termed the $\mathrm{CN}$ vector). These vectors are expressed in polar coordinates, $r$ being the length, $\varphi$ the angle contained in the horizontal plane and $\theta$ the angle in the vertical plane (Fig. 6.4). Polar coordinates are advantageous over Cartesian, because $r$ is essentially constant-vibrations of covalent bond lengths are not observed in these simulations - and otherwise not related to the orientation, hence the analysis can be performed in terms of $\varphi$ and $\theta$ only. By decreasing the number of geometrical parameters, the representation of FESs also becomes simpler. Notwithstanding this, polar coordinates are used only internally, the final results are presented as polar maps in Cartesian coordinates.

After recording all the $\mathrm{CN}$ vectors, a 3D histogram of the distribution of $(\varphi, \theta)$ pairs is built. The reference frame of such histogram is a sphere, based on the definition of the polar coordinates. Sampling over the surface of a sphere suffers from an entropic effect; the likelihood of a certain $\mathrm{CN}$ vector increases when moving from the poles towards the equator, because the area available at the pole is smaller than that available near the equator. This effect has been corrected according to Eq. 6.2.

Finally, the histogram is directly converted into a FES, setting the most frequent state as the ground energy state, according to §1.2.3. The 3D spherical FES is plotted in 2D as the polar aspect of an azimuthal equidistant projection, implemented in the MATPLOTLIB module of Python. ${ }^{26}$ In this type of projection, a sphere is mapped onto a plane parallel to the equator and tangent to the correspondent pole, in such a way that all distances and directions measured from the pole are correct, although other distances, angles, shapes, and sizes are not preserved. As a result, meridians are projected as straight lines from the centre of the projection and parallels are concentric circles equally spaced. This facilitates measuring positions in the projection by directly reading the axis. Although sizes and shapes are not true in the projection, distortion is relatively small, especially if a single hemisphere is projected at a time, as distortion increases away from the centre. 


\subsection{5 tert-Butylammonium carbonate}

\subsubsection{Crystal growth}

After completion of the experiments described in $\S 6.2 .1$, spare ${ }^{t} \mathrm{BA}$ :water samples were stored at $277 \mathrm{~K}$. After a certain period of time, several small transparent crystals had grown in the top half of the vials. Several of those crystals were analysed, to find that they correspond to the carbonate salt of ${ }^{t} \mathrm{BA}$, whose structure, to the best of our knowledge, has not been reported.

\subsubsection{X-ray data collection and processing}

$\mathrm{X}$-ray diffraction data were collected at $150 \mathrm{~K}$ on one of those crystals on a Bruker ApexII CCD diffractometer equipped with a Mo microsource $(\lambda=0.71073 \AA)$. A hemisphere data collection strategy was used, with scan steps of $0.5^{\circ}$ and exposure times of $60 \mathrm{~s}$ per frame. Data were integrated following the strategy outlined in $\S 1.1 .3$, omitting the corrections related to high-pressure samples.

\subsubsection{Structure refinement}

The structure was solved using SHELXs ${ }^{27}$ and subsequently refined by full-matrix least squares against $F^{2}$ using SHELXL v2014/7, ${ }^{10}$ through the GUI ShelXLE. ${ }^{11} \mathrm{~A}$ final $R$ factor of $3.15 \%$ was achieved. For further crystallographic information see Table 6.4.

\subsection{Results and discussion}

\subsubsection{Structure description}

The two hydrates of ${ }^{t} \mathrm{BA}$ reported here, with hydration numbers 5.65 and 5.8, are semi-clathrates, as the ${ }^{t} \mathrm{BA}$ molecules are both enclosed and interacting with the caged water network. Such interaction, by means of $\mathrm{H}$ bonds between the amine group and water molecules, causes two types of defects: substitution, when an amine group replaces a water molecule from the caged network, and intercalation, when the amine group forms 
Table 6.4: Crystallographic data and refinement parameters of pure ${ }^{t} \mathrm{BA}$ and ${ }^{t} \mathrm{BA}^{+}$carbonate.

\begin{tabular}{lll}
\hline & pure ${ }^{\boldsymbol{t}} \mathbf{B A}$ & ${ }^{\boldsymbol{t}} \mathbf{B A}^{+}$carbonate \\
\hline Crystal data & & \\
\hline Chemical formula & $\mathrm{C}_{4} \mathrm{H}_{11} \mathrm{~N}$ & $\left(\mathrm{C}_{4} \mathrm{H}_{12} \mathrm{~N}\right)_{2} \mathrm{CO}_{3}$ \\
Molecular mass $\left(\mathrm{g} \mathrm{mol}^{-1}\right)$ & 73.14 & 208.30 \\
Temperature $(\mathrm{K})$ & $298(2)$ & $150(2)$ \\
Pressure $(\mathrm{GPa})$ & $0.78(5)$ & 0.0001 \\
Crystal system & monoclinic & trigonal \\
Space group & $P 2_{2} 1 / n$ & $R \overline{3} c$ \\
$a(\AA)$ & $5.6799(4)$ & $6.3122(12)$ \\
$b(\AA)$ & $10.1457(9)$ & $6.3122(12)$ \\
$c(\AA)$ & $9.2680(12)$ & $53.215(11)$ \\
$\beta\left({ }^{\circ}\right)$ & $100.611(8)$ & 90 \\
$V\left(\AA^{3}\right)$ & $524.95(9)$ & $1836.2(8)$ \\
$Z$ & 4 & 6 \\
Crystal size $(\mathrm{mm})$ & $\sim 0.15 \times 0.14(\varnothing)$ & $\sim 0.15 \times 0.10 \times 0.05$ \\
\hline
\end{tabular}

\begin{tabular}{lll}
\hline Data collection & \multicolumn{2}{l}{ ( } \\
\hline Radiation wavelength $(\AA)$ & 0.71073 & 0.71073 \\
Absorption correction & Semi-empirical & (using $I$ meas.), SADABS ${ }^{15}$ \\
$T_{\min }, T_{\max }$ & $0.8530,1.0000$ & $0.6233,0.7454$ \\
Number of $[I>2 \sigma(I)]$ reflections: & & \\
- measured & 2529 & 3926 \\
- independent & 288 & 416 \\
- observed & 219 & 354 \\
Completeness $(\%)$ & 69.7 & 97.9 \\
$(\sin \theta / \lambda)_{\max }\left(\AA^{-1}\right)$ & 0.45 & 0.60 \\
$R_{\text {int }}(\%)$ & 1.60 & 2.49 \\
\hline Refinement & & \\
\hline$R\left[F^{2}>2 \sigma\left(F^{2}\right)\right](\%)$ & 11.73 & 3.15 \\
$w R\left(F^{2}\right)(\%)$ & 19.72 & 8.13 \\
$S$ & 1.514 & 1.052 \\
Number of reflections & 288 & 3926 \\
Number of parameters & 50 & 25 \\
Number of restraints & 10 & 0 \\
$\Delta \rho_{\min }, \Delta \rho_{\max }\left(\mathrm{e} \AA^{-3}\right)$ & $-0.084,0.160$ & $-0.181,0.244$ \\
\hline
\end{tabular}

$\mathrm{H}$ bonds with two neighbouring water molecules, forcing them apart and elongating the cage edge. As a result, cages in these semi-clathrates are considerably distorted from regular polyhedra.

\subsubsection{5.65-hydrate}

${ }^{t}$ BA 5.65-hydrate crystallises at $0.76 \mathrm{GPa}$ in an orthorhombic unit cell with parameters $a=17.2082(6) \AA, b=15.6395(6) \AA, c=30.9066(8) \AA$, space group $C m c a$. There are four sites in the asymmetric unit occupied by ${ }^{t} \mathrm{BA}$ molecules, of which only one is fully occupied; the other three lie on special positions, hence containing only a fragment of the ${ }^{t} \mathrm{BA}$ molecule. Each molecule is contained in a different water cage (named $\mathbf{C 1}$ to $\mathbf{C 4}$ ), with no empty cages in the structure (Fig. 6.5). 
Cage $\mathbf{C 1}$ contains the only fully-occupied ${ }^{t} \mathrm{BA}$ molecule of the asymmetric unit, which does not exhibit disorder. The cage exhibits a feature common to other semi-clathrates: the poles are formed by rather regular faces, although some of the faces along the equator are irregular, and their sides are longer than H-bond distances, due to intercalation defects.

Cage C2, and the ${ }^{t} \mathrm{BA}$ molecule it contains, are divided into two symmetric parts by a mirror plane. Similarly to $\mathbf{C} \mathbf{1}$, there are open edges at the equator due to the presence of amine groups $\mathrm{H}$ bonded to water molecules belonging to the cage.

Only a quarter of cage C3 lies inside the asymmetric unit, the rest of it being generated by a mirror plane and a two-fold rotation axis perpendicular to it. The corresponding ${ }^{t} \mathrm{BA}$ molecule is fully generated by the mirror plane, while the rotation axis creates another entire molecule in a different orientation and position, leading to disorder. All the edges of this cage exhibit lengths corresponding to $\mathrm{H}$ bonds, but there are substitutional defects; two water molecules are affected by the disorder of the ${ }^{t} \mathrm{BA}$ and each one can coexist with only one of the two disordered orientations. The lack of intercalation defects allows for a cage with more regular faces.

Cage $\mathbf{C 4}$ resembles $\mathbf{C 3}$, in that is also generated by a mirror plane and a two-fold axis, although the ${ }^{t} \mathrm{BA}$ molecule inside this cage is only orientationally (and not positionally) disordered because the central carbon atom lies directly on the inversion center-where the rotation axis intersects the mirror plane. In this case both substitutional and intercalation defects are present.

\subsubsection{5.8-hydrate}

The 5.8-hydrate of ${ }^{t} \mathrm{BA}$ crystallises at $0.61 \mathrm{GPa}$ in a monoclinic cell with parameters $a=12.5091(13) \AA, b=22.213(3) \AA, c=18.766(2) \AA$, $\beta=92.985(3)^{\circ}$, space group $P 2_{1} / n$. It is also a semi-clathrate and can be described in terms of five different cages (named D1 to D5), each containing a fully occupied, although sometimes disordered, ${ }^{t} \mathrm{BA}$ molecule (Fig. 6.6). 
Figure 6.5: Top: Perspective view along the $a$ axis of the asymmetric unit of ${ }^{t} \mathrm{BA}$ 5.65-hydrate. ${ }^{t} \mathrm{BA}$ molecules are represented as grey spheres. $\mathrm{H}$ bonds between water molecules are plotted as solid lines, coloured to differentiate the four cages. Cages have been expanded out of the asymmetric unit for clearer representation. Bottom: four independent water cages in the structure of ${ }^{t} \mathrm{BA} 5.65$ hydrate. ${ }^{t} \mathrm{BA}$ molecules are depicted by solids lines, colour-coded according to the atom type $(\mathrm{C}$ in dark grey, $\mathrm{N}$ in blue, and $\mathrm{H}$ in light grey). $\mathrm{H}$ bonds between water molecules making up the cages are represented by thick lines, solid at the front and dashed at the back to give a sense of perspective, each cage coloured differently. Cage edges longer than $\mathrm{H}$ bond distances are depicted as thin dashed lines. $\mathrm{H}$ bonds between amine groups and water molecules are shown as thin red dashed lines. Mirror planes are presented as semi-transparent yellow planes; two-fold rotation axis are shown as green lines; cage vertices corresponding to water molecules affected by substitutional defects are highlighted as orange circles.
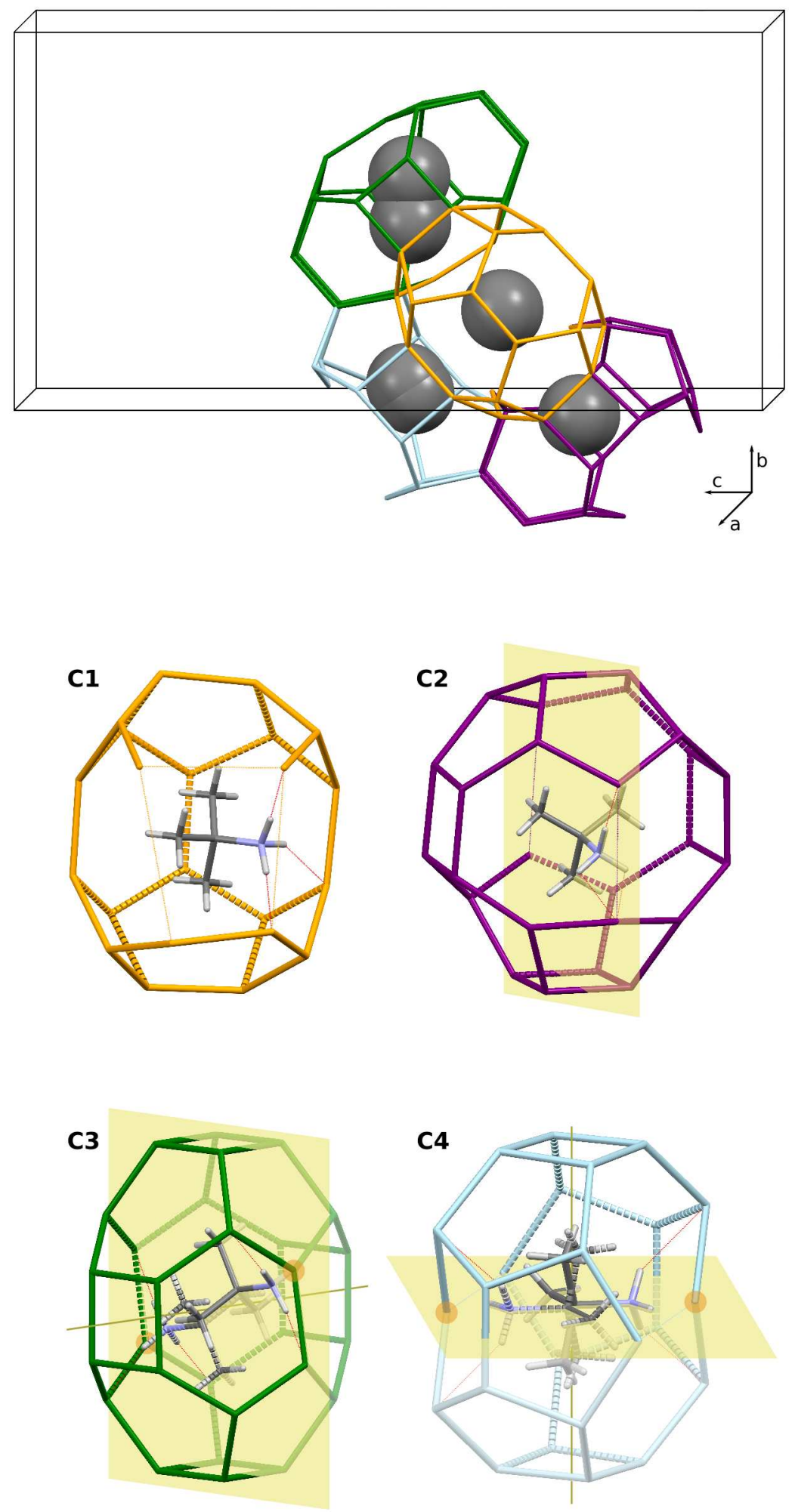


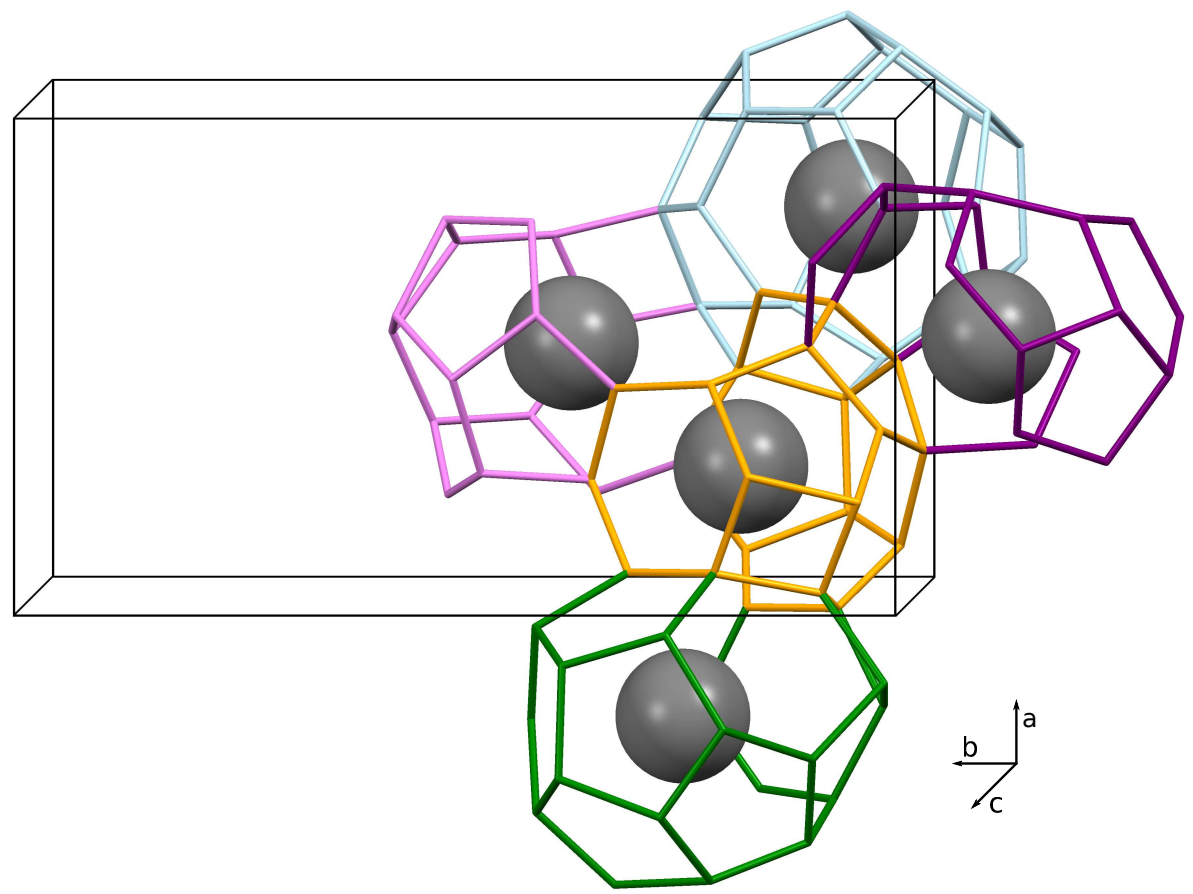

Figure 6.6: Top: Perspective view along the $c$ axis of the asymmetric unit of ${ }^{t} \mathrm{BA}$ 5.8-hydrate. ${ }^{t} \mathrm{BA}$ molecules are represented as grey spheres. $\mathrm{H}$ bonds between water molecules are plotted as solid lines, coloured to differentiate the five cages. Cages have been expanded out of the asymmetric unit for clearer representation. Bottom: five independent water cages in the structure of ${ }^{t} \mathrm{BA}$ 5.8-hydrate. ${ }^{t} \mathrm{BA}$ molecules are depicted by solids lines, colour-coded according to the atom type $(\mathrm{C}$ in dark grey, $\mathrm{N}$ in blue, and $\mathrm{H}$ in light grey). $\mathrm{H}$ bonds between water molecules making up the cages are represented by thick lines, solid at the front and

D1

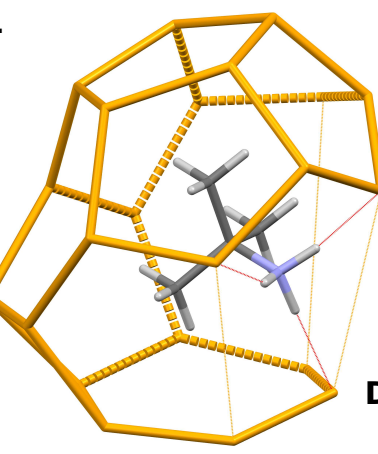

D3

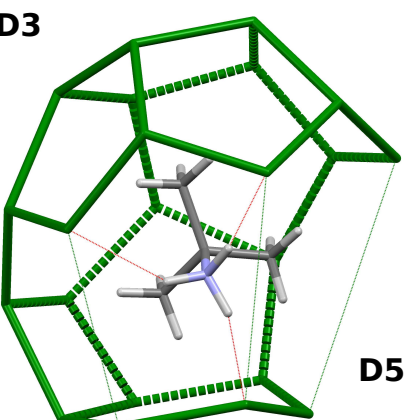

D4

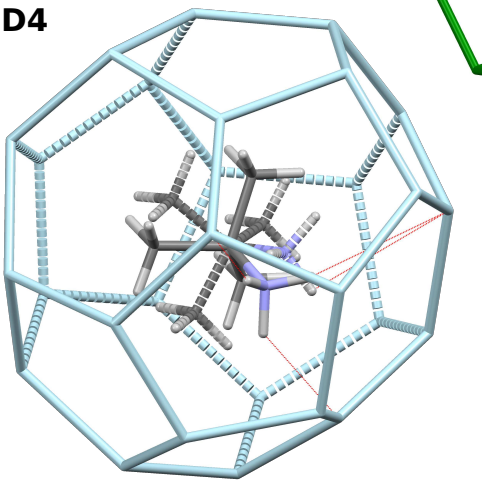

D2

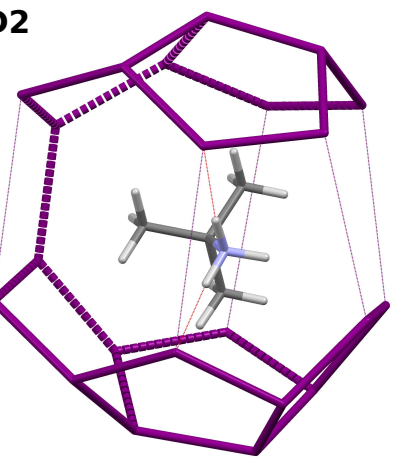

D5

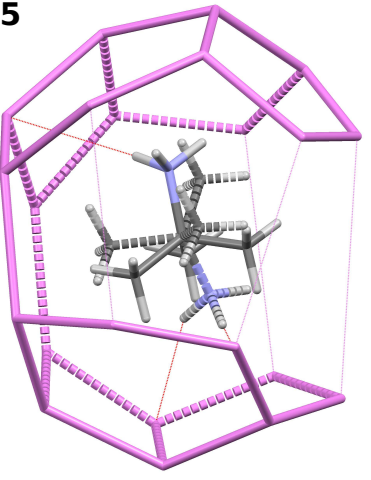

dashed at the back to give a sense of perspective, each cage coloured differently. Cage edges longer than $\mathrm{H}$ bond distances are depicted as thin dashed lines. $\mathrm{H}$ bonds between amine groups and water molecules are shown as thin red dashed lines. 
Cage D1 exhibits a feature similar to many of the cages described for the previous structure: the poles are formed by faces close to a regular polygon, while the cage is open around the equator due to the intercalation of the amine group. This feature is present in all of the cages, with the exception of $\mathbf{D} 4$.

The effect of intercalation defects in cage D2 is more dramatic, as there is a single $\mathrm{H}$ bond linking both poles of the cage, with the rest of the edges being considerably larger.

Cage D3 is very similar to D1.

Cage D4 is the largest of all, which allows for a ${ }^{t} \mathrm{BA}$ molecule to be contained inside without causing defects. All edges correspond to $\mathrm{H}$ bonds, based on their lengths, although this does not imply that the cage is closed, as not all edges can coexist with the $\mathrm{H}$ bonds formed between the amine group and the water network. The ${ }^{t} \mathrm{BA}$ molecule inside this cage is equally disordered between two possible orientations.

Cage D5 resembles D2, as there are only two $\mathrm{H}$ bonds linking both poles, although in this case the ${ }^{t} \mathrm{BA}$ molecule is disordered inside the cage. One orientation is populated $80 \%$ of the time, while the other only $20 \%$.

\subsubsection{Pure tert-butylamine form II}

The structure of ${ }^{t} \mathrm{BA}$ form II, crystallised at $0.78 \mathrm{GPa}$, has already been introduced in $\S 6.2 .4 .3$. The unit cell, with parameters $a=5.6799(4) \AA$, $b=10.1457(9) \AA, c=9.2680(12) \AA, \beta=100.611(8)^{\circ}$, space group $P 2_{1} / n$, contains four symmetry-equivalent ${ }^{t} \mathrm{BA}$ molecules. Molecules have been described to be disordered between four possible orientations, with an equal likelihood of the nitrogen atom being in each of the four vertices of the tetrahedral geometry. Based on single-crystal X-ray diffraction only, it is not possible to determine whether the molecules are in fact disordered, or if the data simply does not allow to distinguish between carbon and nitrogen atoms. Complete rotational freedom of the ${ }^{t} \mathrm{BA}$ molecules is another possibility, although the refinement does not support it. 
The packing can be described by layers of ${ }^{t} \mathrm{BA}$ molecules stacked along the $c$ axis (Fig. 6.7). Each molecule is surrounded by another six in the same layer, and three other above and below. The separation between molecules, measured as distance between the central carbon atoms, varies between 5.342 and $6.329 \AA$, with an average value of $5.785 \AA$.

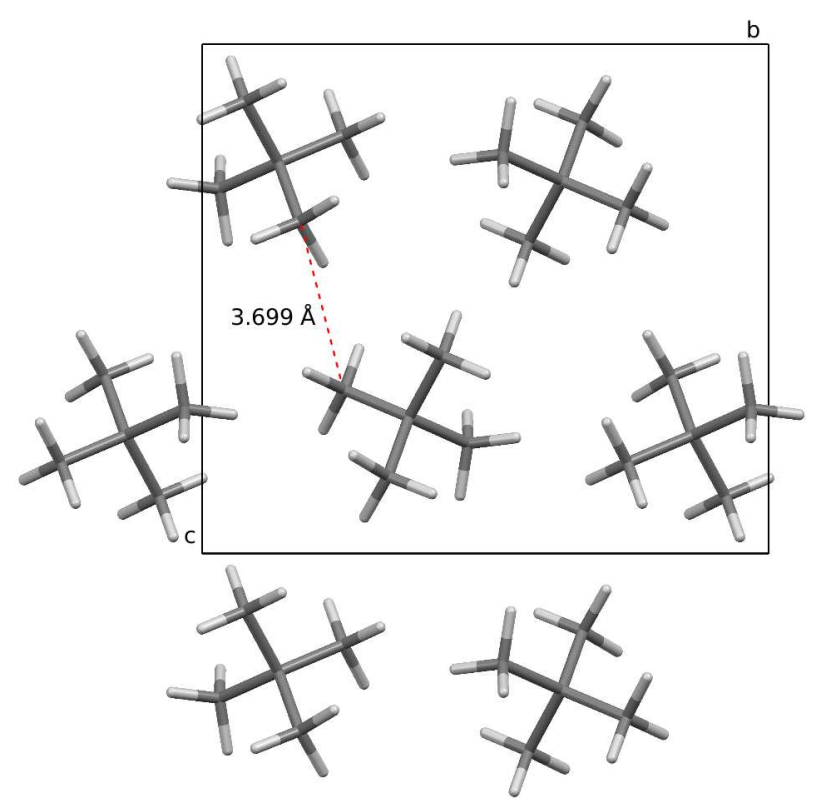

Figure 6.7: Packing arrangement of ${ }^{t} \mathrm{BA}$ form II at $0.78 \mathrm{GPa}$, view along the $a$ axis. Three layers, parallel to the $a b$ plane, have been depicted. Molecules in the top and bottom layer are aligned with the intermolecular spaces in the middle layer, resembling spheres close packing. The shortest possible $\mathrm{H}$ bond in the structure (as nitrogen-nitrogen distance) is indicated by a dashed red line.

Further analysis shows that the shortest nitrogen-nitrogen interaction is $3.699 \AA$, allowing only for weak $\mathrm{H}$ bonds. ${ }^{28}$ Nevertheless, the presence of such $\mathrm{H}$ bonds might be sufficient to limit the rotational freedom of the molecules to a certain set of orientations. A deeper study of the disorder in this structure, based on MD simulations, is presented in $\S 6.3 .3$.

\subsubsection{4 tert-Butylammonium carbonate}

The crystal structure of the unhydrated ${ }^{t} \mathrm{BA}^{+}$carbonate has a trigonal unit cell with parameters $a=6.3122(12) \AA$ and $c=53.215(11) \AA$, space group $R \overline{3} c$. The assymmetric unit contains a sixth of a formula unit: a third of a ${ }^{t} \mathrm{BA}^{+}$cation and a sixth of a carbonate anion. The structure consists of sandwich layers - anions surrounded above and below by cations - parallel to the $a b$ face of the unit cell (Fig. 6.8). Each carbon- 
Figure 6.8: Layered packing of ${ }^{t} \mathrm{BA}^{+}$carbonate, view along the $a$ axis. Layers are parallel to the $a b$ plane and are stacked along the $c$ axis. Only a third of the cell has been represented. The orange areas correspond to regions governed by $\mathrm{H}$ bonds or ionic interactions. The blue area corresponds to the region governed by London dispersion forces.

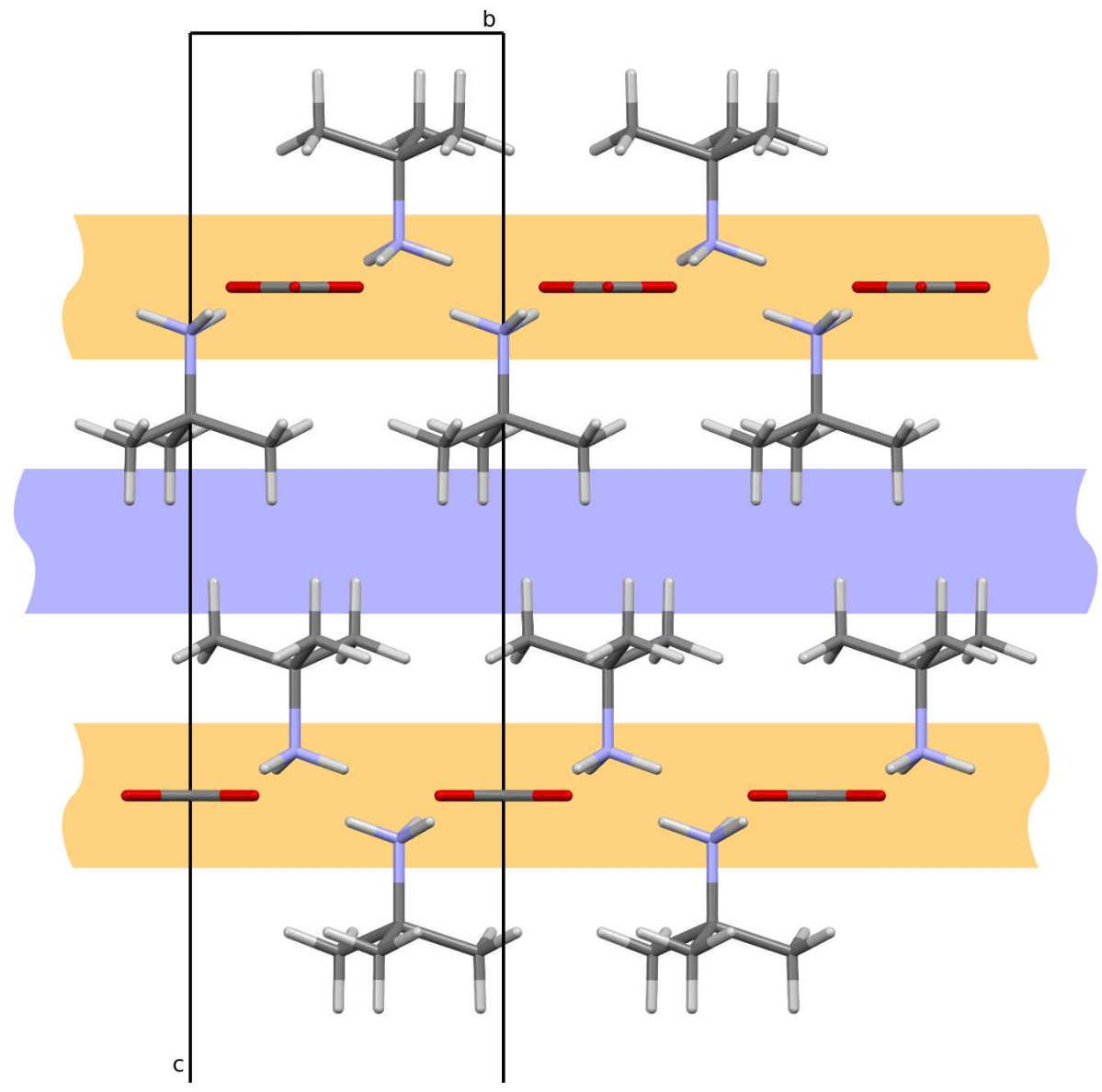

ate anion is linked to six ${ }^{t} \mathrm{BA}^{+}$units, two per oxygen atom, through the corresponding amine groups; the relative degree of covalent and ionic character of these links has not been evaluated. The orientation of the cations is such that the carbon-nitrogen bond is perfectly perpendicular to the plane containing the carbonate anions, which is in turn perfectly parallel to the $a b$ plane of the unit cell. Sandwich layers are stacked along the $c$ axis held together by London dispersion forces between the tert-butyl groups.

The crystallisation ${ }^{t} \mathrm{BA}^{+}$carbonate is remarkable, as crystals grew out of solution, on the top half of vials containing ${ }^{t} \mathrm{BA}$ : water mixtures stored at $277 \mathrm{~K}$, without any source of carbonate intendedly added to the medium. The most reasonable explanation is that ${ }^{t} \mathrm{BA}$ accumulated in the vapour phase inside the close vial, due to its high vapour pressure $\left(0.388 \mathrm{~atm}\right.$ at $\left.20^{\circ} \mathrm{C}\right)$. Then an acid-base reaction must have occurred with the $\mathrm{CO}_{2}$ naturally occurring in the air trapped inside the vial. 
${ }^{t} \mathrm{BA}^{+}$carbonate formed as a result of this reaction and crystallised attached to the walls of the vials, favoured by the low temperatures. The natural amount of $\mathrm{CO}_{2}$ is in fact sufficient to grow small crystals, as observed here. This process is not unknown, as it constitutes the basis for industrial $\mathrm{CO}_{2}$ removal from gas streams. ${ }^{29}$

\subsubsection{Crystalline phases in the water-tert-butylamine system}

There are eleven known crystalline phases in the water ${ }^{t} \mathrm{BA}$ system, including two anhydrous forms and the three structures reported in this work (Table 6.5). Six of these structures were reported by Dobrzycki et al., along with a relationship for the variation of density with the hydration number of each phase. ${ }^{5}$ Such relationship takes the form of an inverted Morse potential:

$$
\rho(N)=s-d_{e}\left(1-\mathrm{e}^{-a\left(N-r_{e}\right)}\right)^{2}
$$

Were $\rho$ is the density of each crystalline phase $N$ (denoted by its hydration number), normalised with respect to ice $\mathrm{Ih}, s$ is $1.076 \mathrm{~g} / \mathrm{cm}^{3}, d_{e}$ is $0.163 \mathrm{~g} / \mathrm{cm}^{3}, a$ is 0.179 , and $r_{e}$ is 4.32 . Values for the four parameters $s$, $d_{e}, a$, and $r_{e}$ were obtained by fitting the equation to the experimental densities. ${ }^{5}$ The validity of the proposed relationship was confirmed by its ability to predict the densities of the tert-butanol water clathrates. ${ }^{5}$

An important observation by Dobrzycki et al. is the existence of a region of hydration numbers between 1 and $7^{1 / 4}$ for which no crystal structures could be obtained. The maximum density, predicted for a potential 4.32-hydrate, falls in this region, hence structures with hydration numbers between 4 and 5 are expected to show the most efficient packings. It was stated that "this gap should almost be expected because it represents a fuzzy guest/host transition area without nucleation/crystal growth", ${ }^{5}$ based on the possible interactions between water molecules in the structure. For the lowest hydration numbers, water molecules cannot interact between them, as they are located in the intermolecular spaces in the packing of ${ }^{t} \mathrm{BA}$ molecules. In theory, as the hydration number in- 
Table 6.5: Crystalline phases of the water $-{ }^{t} \mathrm{BA}$ system.

Table 6.6: Parameters of Eq. 6.3, as determined originally by Dobrzycki et al. (top) and redetermined in this work (bottom). $s$ and $d_{e}$ in $\mathrm{g} / \mathrm{cm}^{3}, a$ and $r_{e}$ are dimensionless.

\begin{tabular}{lr}
\hline $\boldsymbol{s}$ & $1.076(11)$ \\
$\boldsymbol{d}_{\boldsymbol{e}}$ & $0.163(22)$ \\
$\boldsymbol{a}$ & $0.179(19)$ \\
$\boldsymbol{r}_{\boldsymbol{e}}$ & $4.32(31)$ \\
\hline $\boldsymbol{s}$ & $1.079(9)$ \\
$\boldsymbol{d}_{\boldsymbol{e}}$ & $0.166(22)$ \\
$\boldsymbol{a}$ & $0.179(21)$ \\
$\boldsymbol{r}_{\boldsymbol{e}}$ & $4.31(33)$ \\
\hline
\end{tabular}

\begin{tabular}{|c|c|c|c|c|c|}
\hline$N$ & Structure type & $\boldsymbol{T}$ or $\boldsymbol{P}^{b}$ & $\begin{array}{c}\rho \\
\left(\mathrm{g} / \mathbf{c m}^{3}\right)\end{array}$ & $\begin{array}{l}\text { Norm. } \rho \\
\left(\mathrm{g} / \mathrm{cm}^{3}\right)\end{array}$ & Authors \\
\hline 0 & Anhydrate & $133 \mathrm{~K}$ & 0.860 & 0.857 & Dobrzycki et al. ${ }^{5}$ \\
\hline 0 & Anhydrate & $0.78 \mathrm{GPa}$ & 0.925 & - & This work \\
\hline 0.25 & Simple hydrate & $273 \mathrm{~K}$ & 0.884 & 0.884 & Dobrzycki et al. ${ }^{5}$ \\
\hline 1 & Simple hydrate & $173 \mathrm{~K}$ & 0.975 & 0.975 & Dobrzycki et al. ${ }^{5}$ \\
\hline 5.65 & Semi-clathrate & $0.76 \mathrm{GPa}$ & 1.117 & 1.059 & This work \\
\hline 5.8 & Semi-clathrate & $0.61 \mathrm{GPa}$ & 1.133 & 1.086 & This work \\
\hline 7.25 & Semi-clathrate & $248 \mathrm{~K}$ & $1.038^{c}$ & 1.041 & Stäben and Mootz ${ }^{4}$ \\
\hline 7.75 & Semi-clathrate & $203 \mathrm{~K}$ & 1.035 & 1.041 & Dobrzycki et al. ${ }^{5}$ \\
\hline 9.75 & Semi-clathrate ${ }^{a}$ & $271 \mathrm{~K}$ & $1.005^{c}$ & 1.010 & McMullan et al. ${ }^{2}$ \\
\hline 11 & Semi-clathrate & $250 \mathrm{~K}$ & 1.004 & 1.014 & Dobrzycki et al. ${ }^{5}$ \\
\hline 17 & Semi-clathrate & $243 \mathrm{~K}$ & 0.932 & 0.940 & Dobrzycki et al. ${ }^{5}$ \\
\hline
\end{tabular}

${ }^{a}$ Although formally a semi-clathrate, this structure does not exhibit permanent defects in the water network, hence it resembles a true clathrate.

${ }^{b}$ Structures for which a temperature is given were obtained at ambient pressure. Structure for which a pressure is given were obtained at ambient temperature.

${ }^{c}$ From the redeterminations by Dobrzycki et al. rather than the original author.

creases, the water network changes from this $0 \mathrm{D}$ state (0.25-hydrate) to chains (1D, mono-hydrate), layers (2D), and finally cages (3D, 7.25- and higher hydrates). In practice, considering the whole continuum, a layered water network has only been observed for tert-butanol hydrates. ${ }^{30}$ For ${ }^{t} \mathrm{BA}$, a $2 \mathrm{D}$ arrangement has not been observed; the gap in hydration numbers probably exists because the jump from a $2 \mathrm{D}$ to a $3 \mathrm{D}$ arrangement requires a considerable increase in water molecules.

The densities of the two high-pressure semi-clathrates reported in this work have been normalised (§6.3.2.1) and the relationship between the density and the hydration number has been recalculated using FITYK $^{31}$ (Fig. 6.9). The new parameters for Eq. 6.3 are in Table 6.6, along with the original parameters. Overall, only a small change is observed, meaning that the two new structures behave as expected.

The use of high pressure shows that it is possible to obtain crystal structures which are closer to the maximum possible density. Nevertheless, the transition between simple hydrates and clathrate-like structures is still unclear. Without neglecting the idea of the stability of the structure associated with the water network proposed by Dobrzycki et al., the 5.65- and 5.8-hydrates point towards a more complex scenario, in which a 3D water network still exists at low hydration numbers, but cannot be interpreted without considering the intercalation of amine groups. 


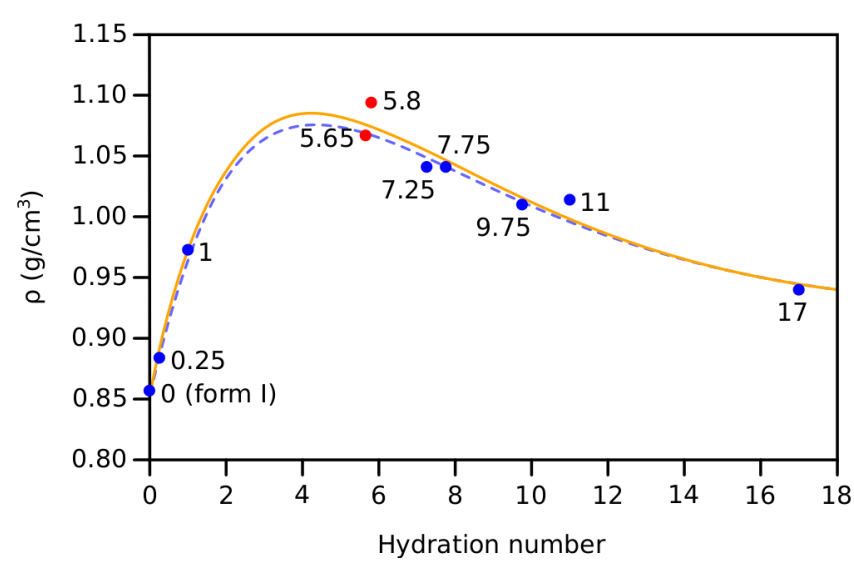

Form II of pure ${ }^{t} \mathrm{BA}$ has not been included in this calculation, because there is not an appropriate reference against which to normalise its density at ambient pressure.

\subsubsection{Density normalisation}

The densities of the structures determined by Dobrzycki et al. ${ }^{5}$ (Table 6.5) were normalised to $173 \mathrm{~K}$ taking non-deuterated ice $\mathrm{Ih}$ as a reference. Unit cell volumes at each temperature of interest were calculated using the $V-T$ equation of state determined by Röttger et al., ${ }^{32}$ normalisation factors $f_{T}$ were calculated according to Eq. 6.4, and then the densities were normalised according to Eq. 6.5.

A similar procedure was applied to normalise the densities of the high-pressure 5.65- and 5.8-hydrates to ambient pressure. A quadratic expression for the pressure dependence of the density of non-deuterated ice Ih was proposed by Gagnon et al. based on Brillouin spectroscopy data collected at $237.65 \mathrm{~K}$ up to $0.28 \mathrm{GPa} .{ }^{33}$ Densities were calculated using this relationship, they were converted into volumes afterwards, and all volumes were finally normalised to $173 \mathrm{~K}$ :

$$
\begin{gathered}
\rho(173)=f_{T} \rho(T) \Rightarrow \frac{m}{V(173)}=f_{T} \frac{m}{V(T)} \Rightarrow \\
V(173)=\frac{1}{f_{T}} V(T)
\end{gathered}
$$

Normalisation factors calculated according to Eq. 6.6 have been computed using these data (Table 6.7).
Figure 6.9: Variation of density with the hydration number of the different crystalline phases of the ${ }^{t} \mathrm{BA}$ :water system. Each data point is labelled with the corresponding hydration number; the two HP semi-clathrates are highlighted in red. The orange curve corresponds to the recalculated relationship; the dashed blue curve to the original calculation. ${ }^{5}$

$$
\begin{gathered}
f_{T}=\frac{V(T)}{V(173)} \\
\rho(173)=f_{T} \rho(T) \\
f_{P}=\frac{V(P)}{V(0)}
\end{gathered}
$$


6. High pressure study of the water-tert-butylamine system

Table 6.7: Normalisation factors calculated from data corresponding to non-deuterated ice $\mathrm{Ih}$ at $237.65 \mathrm{~K}$.

\begin{tabular}{cccccc}
\hline $\boldsymbol{P}(\mathbf{G P a})$ & $\boldsymbol{\rho}\left(\mathrm{g} / \mathbf{c m}^{3}\right)$ & $\boldsymbol{V}\left(\AA^{3}\right)$ & $\boldsymbol{f}_{\boldsymbol{T}}$ & $\boldsymbol{T}$-norm. $\boldsymbol{V}\left(\AA^{3}\right)$ & $\boldsymbol{f}_{\boldsymbol{P}}$ \\
\hline 0.00 & 0.9228 & 129.67 & 1.0076 & 128.69 & \\
0.61 & 0.9783 & 122.32 & 1.0076 & 121.40 & 0.943 \\
0.76 & 0.9897 & 120.91 & 1.0076 & 119.99 & 0.932 \\
\hline
\end{tabular}

The normalisation factors in Table 6.7 have been used for all purposes in this study. Nevertheless, acknowledging the inherent risk of extrapolating the polynomial curve from 0.28 to 0.61 and $0.76 \mathrm{GPa}$, two tests have been performed to ensure that the previous procedure performs appropriately.

A $P-V$ equation of state was proposed by Strässle et al. for deuterated ice Ih at $145 \mathrm{~K} .{ }^{34}$ These data cannot be used to compute the normalisation factors in this study, because the compressibilities of deuterated and non-deuterated ice Ih are slightly different and the isotope effect has not been evaluated at the pressures used in this study. ${ }^{35}$ Notwithstanding, the isotope effect is expected to be small and the $P$ $V$ relationship for the deuterated compound takes the form of a Murnaghan equation of state, which can be extrapolated more reliably than a quadratic equation. Normalisation factors were calculated using these data, to check that they do not differ considerably from those calculated previously. This indicates that the extrapolation on the quadratic equation does not introduce large errors. The same procedure as before was used (Eqs. 6.7 and 6.6), although the step involving the densities was not required. The normalisation factors obtained were indeed very close to the ones obtained from non-deuterated ice Ih (Table 6.8). See Fig. 6.10 for a comparison of both equations of state.

As second test, a quadratic equation of the same form as proposed by Gagnon et al. was fitted to 11 data points generated from the Murnaghan $P-V$ equation of state. Both the quadratic and Murnaghan equations overlap below $0.5 \mathrm{GPa}$, but diverge at higher pressures, albeit just slightly (Fig. 6.10). Translating this observation into the data for deuterated ice Ih, it is safe to assume that, in the absence of a more robust equation, the error in the normalisation factors due to the extrapolation of the quadratic equation should be small. 
6.3. Results and discussion

\begin{tabular}{ccccc}
\hline $\boldsymbol{P}(\mathbf{G P a})$ & $\boldsymbol{V}\left(\AA^{3}\right)$ & $\boldsymbol{f}_{\boldsymbol{T}}$ & $\boldsymbol{T}$-norm. $\boldsymbol{V}\left(\AA^{3}\right)$ & $\boldsymbol{f}_{\boldsymbol{P}}$ \\
\hline 0.00 & 128.18 & 1.0035 & 127.73 & \\
0.61 & 121.69 & 1.0035 & 121.27 & 0.949 \\
0.76 & 120.43 & 1.0035 & 120.01 & 0.940 \\
\hline
\end{tabular}

Table 6.8: Normalisation factors calculated from data corresponding to deuterated ice Ih at $237.65 \mathrm{~K}$.

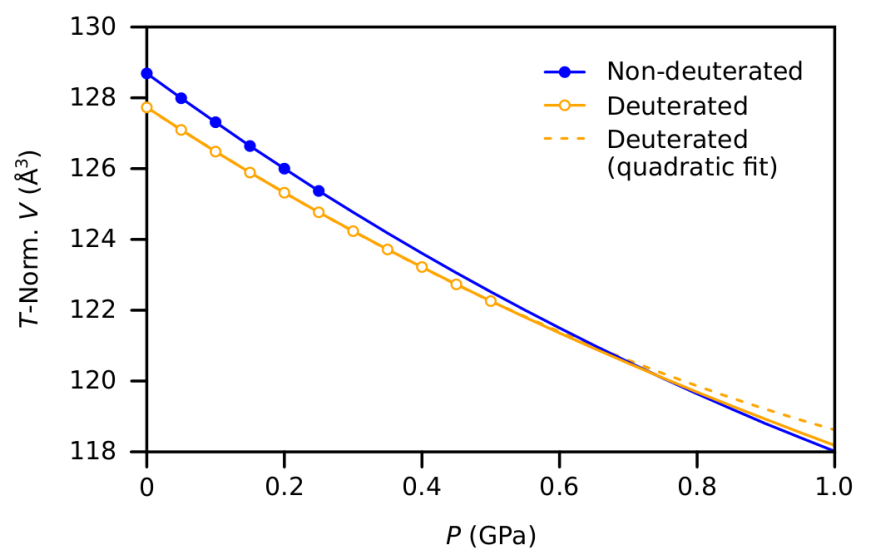

Figure 6.10: Compressibility data of deuterated and non-deuterated ice Ih. Data points are represented in the interval for which data is available (up to $0.28 \mathrm{GPa}$ for non-deuterated ice and up to $0.5 \mathrm{GPa}$ for deuterated ice). The curves are drawn to $1.0 \mathrm{GPa}$ as solid lines to show the crossing point at $0.75 \mathrm{GPa}$. The quadratic fit of the data for deuterated ice is shown as a dashed line.

\subsubsection{Computational interpretation of pure tert-butylamine form II}

Simulations were initially performed using two different force fields, GROMOS and OPLS. The latter was considered a better choice, and after modifying the atomic charges, was used in the production runs. Each simulation was repeated five times, at the exact same conditions and starting from the same setup. Fig. 6.11 shows a zenithal view of the FESs obtained with the three different force fields, after combining the five repeated simulations. Lighter areas correspond to energy minima, while darker areas should be interpreted as energy barriers. As explained in $§ 6.2 .4 .4,{ }^{t} \mathrm{BA}$ molecules are orientationally disordered, but there is no indication that their position inside the unit cell changes significantly.
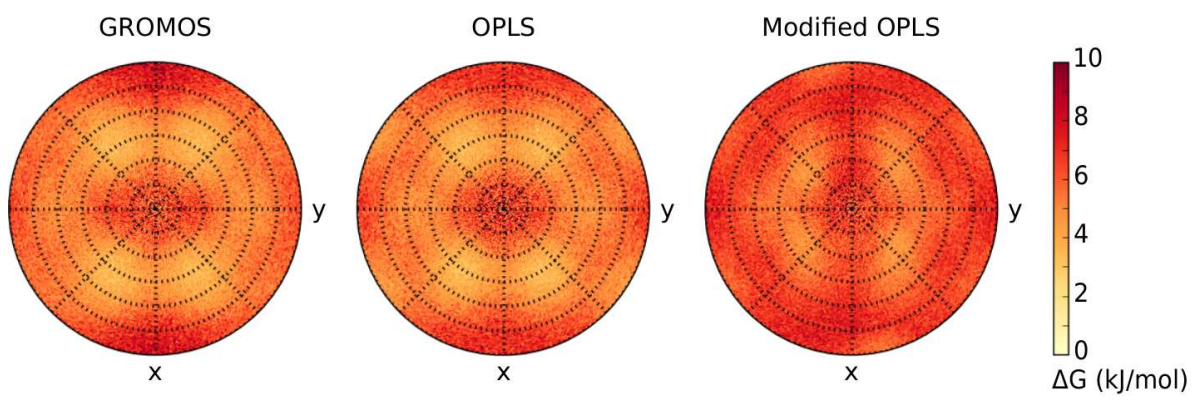

Figure 6.11: FESs of ${ }^{t} \mathrm{BA}$ form II at $0.78 \mathrm{GPa}$, view along $z$, calculated from five simulations, using three different force fields, GROMOS, OPLS and modified OPLS. 
Therefore, it is possible to interpret these graphs as the projection of a hemisphere, with the central carbon atom of a ${ }^{t} \mathrm{BA}$ molecule sitting at the centre of the sphere and the amine being potentially located at any of the low energy regions. The results from GROMOs and OPLS are nearly identical, while the use of the modified opLs force field suggests a considerably more restricted set of possible orientations. Fig. 6.12 shows similar FESs, obtained from just one simulation instead of five, using either GROMOS or OPLS as a force field. The latter produces a better sampling of the high-energy orientations, while points in the graph obtained using GROMOs are more concentrated at the low-energy regions. Although this difference is compensated by computing the graphs from several simulations, OPLS was chosen as it has the potential advantage of generating more detailed FESs.

Figure 6.12: FESs of ${ }^{t} \mathrm{BA}$ form II at $0.78 \mathrm{GPa}$, view along $z$, calculated from a single simulation, using two different force fields, GROMOS and OPLS.
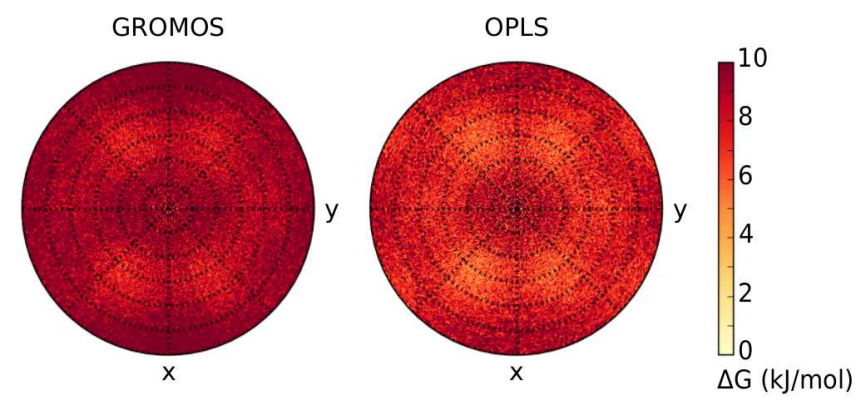

The FES obtained from the simulations at $0.78 \mathrm{GPa}$ using the modified OPLS force field is presented in Fig. 6.13. The results indicate that the amine group is preferentially located in eight different orientations. Energy barriers between different orientations vary between 6 and $9 \mathrm{~kJ} / \mathrm{mol}$. Weak $\mathrm{H}$ bonds as observed in this structure are expected to exhibit energies in the order of less than $20 \mathrm{~kJ} / \mathrm{mol},{ }^{28}$ hence dynamic disorder in the solid state cannot be discarded on the basis of these results.

A reconstruction of the eight possible positions in fractional coordinates is shown in Fig. 6.14, superimposed with the four possible positions obtained from X-ray diffraction for comparison. Although each position is represented by a single point, it covers in fact a small area, $15^{\circ}$ wide in all directions. Given that the radius of the sphere in which the ${ }^{t} \mathrm{BA}$ molecules are inscribed is $1.47 \AA$ (average length of the carbon-nitrogen 

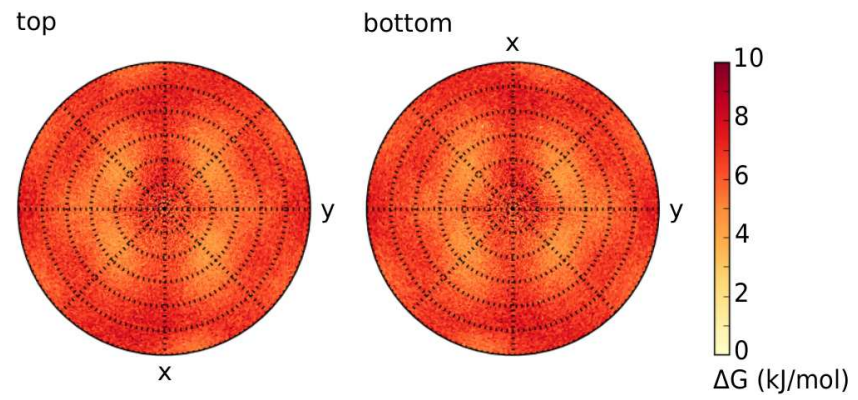

Figure 6.13: FESs of ${ }^{t} \mathrm{BA}$ form II at $0.78 \mathrm{GPa}$, view along $z$ from above and below, calculated from five simulations using the modified OPLS force field.

bonds), those $15^{\circ}$ correspond to a circle perpendicular to the carbonnitrogen bond $0.768 \AA$ in diameter. Each $\mathrm{N}$ atom in the crystal structure can be associated to a different preferred position from the simulation, with distances varying from 0.236 to $0.787 \AA$, which indicates a good agreement between both structures. The four additional positions predicted by MD probably correspond to the disorder that cannot be modelled from the experimental data. In fact, if all ${ }^{t} \mathrm{BA}$ molecules within the unit cell were to be reoriented as to match one of the eight possible orientations determined from MD, nitrogen-nitrogen distances between neighbouring molecules will range between 2.5 and $3.5 \AA$, resulting in stronger $\mathrm{H}$ bonds.

FESs determined from all the molecules inside the simulation box allow to recreate an average structure, but do not inform about its dynamic behaviour. The same analysis script was applied to individual molecules within a simulation; Fig. 6.15 shows FESs for three independent molecules (all the molecules were inspected and show similar behaviour). Differently from previous FESs, sampling from just a single molecule is notably restricted to specific areas, which vary from molecule to molecule. This indicates that each ${ }^{t} \mathrm{BA}$ molecule exhibits a single preferred orientation, with a certain degree of libration $\left(\sim 15^{\circ}\right.$ in
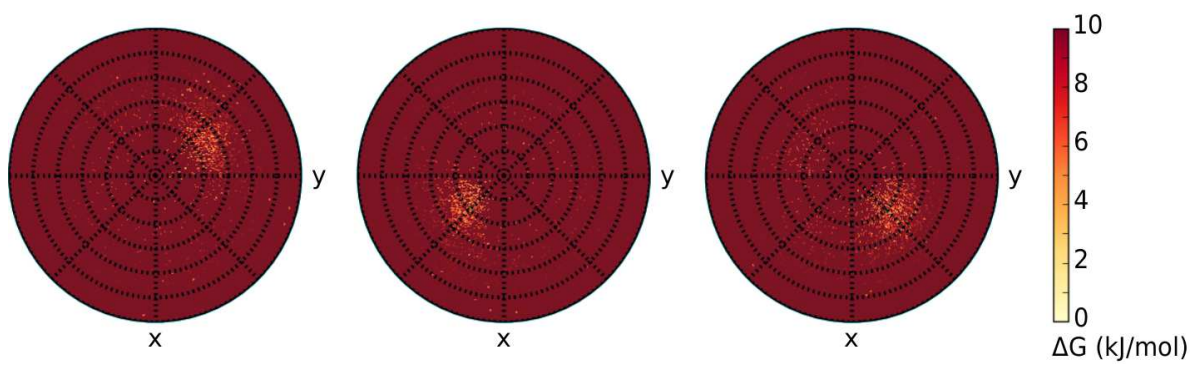

Figure 6.15: FESs of ${ }^{t} \mathrm{BA}$ form II at $0.78 \mathrm{GPa}$, zenithal view along $z$, calculated for three different individual molecules of the simulation box. 
all directions), that is, there is no dynamic disorder in the structure. Reorentation occurs, as evidenced by spots scattered outside the preferred areas, but can be considered a rare event. Based on this, the structure of ${ }^{t}$ BA form II can be catalogued as an orientational glass, ${ }^{6,7}$ that is, only the central carbon atom of each ${ }^{t} \mathrm{BA}$ molecule is subject to the space group symmetry $\left(P 2_{1} / n\right)$, while each molecule is orientated differently. This successfully explains the concerns during the refinement expressed in $\S 6.2 .4 .3$.

Another aspect of the structure addressed in $\S 6.2 .4 .1$ concerns the compression of a crystal of ${ }^{t} \mathrm{BA}$ over $0.78 \mathrm{GPa}$, which inevitably leads to loss of crystal quality. In order to check the effect of compression, two additional simulations were performed at 0.40 and 1.20 GPa. FESs, averaged for all molecules over five simulations, obtained at the four different pressures, are reported in Fig. 6.16. At ambient pressure all orientations are equally likely, that is, the material is in its liquid state, as expected. As pressure increases, preferred orientations become more and more restricted. At $1.20 \mathrm{GPa}$ the eight preferred orientations for the amine group have become four (half of them are visible in the graphs, the other half are visible in the remaining hemisphere, which is symmetric and has not been represented here) and are slightly shifted with respect to the lower pressures. Despite such preferred orientations being lower in energy than those at lower pressure, there is a large connected region at $\sim 5 \mathrm{~kJ} / \mathrm{mol}$ around them, which indicates that the degree of orientational disorder is even larger than before. The most likely explanation is that at this pressure ${ }^{t} \mathrm{BA}$ molecules get closer, and due to increasing repulsive interactions not all molecules can reorient into the most favourable orientation. This increased degree of internal disorder might also explain why it was not possible to observe X-ray diffraction from samples compressed over $1 \mathrm{GPa}$.

\subsection{Conclusions and outlook}

The crystal structures of three new solid phases of ${ }^{t} \mathrm{BA}$ have been determined, increasing the total number of known phases in the water $-{ }^{t} \mathrm{BA}$ 

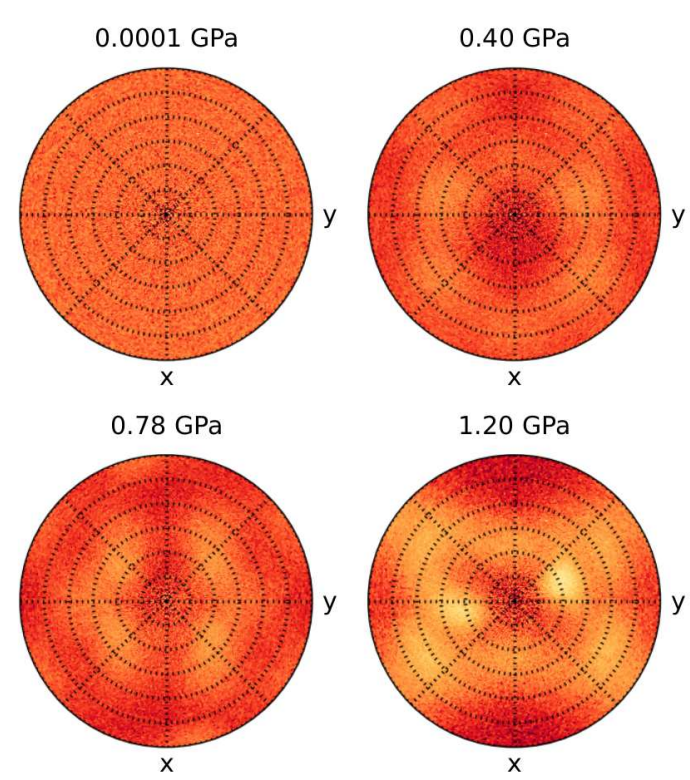

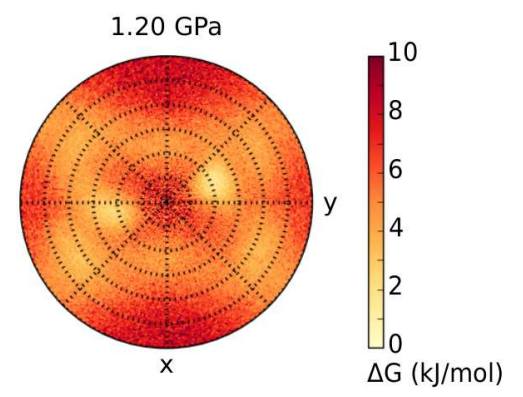

system to ten. Two of these phases are semi-clathrate structures, in which water molecules form an irregular network of cages that host ${ }^{t} \mathrm{BA}$ molecules. The importance of these semi-clathrates stems from the fact that they exhibit the highest density among all the phases, very close to the predicted maximum density of the system. On the basis of experiments conducted at ambient pressure and low temperature, it was previously considered that crystal growth in this high-density region was hindered due to the impossibility of forming a $2 \mathrm{D}$ water network which could host the ${ }^{t} \mathrm{BA}$ molecules. By means of in-situ high-pressure crystallisation it has been shown that crystallisation in said region is in fact possible, considerably reducing the size of the gap in which a $2 \mathrm{D}$ arrangement of water molecules might appear.

The structure of a new polymorph of pure ${ }^{t} \mathrm{BA}$ has also been reported, showing that cooling and compression of the neat substance lead to two different phases. The HP form II has been characterised as an orientational glass. Single-crystal X-ray diffraction allows to determine the overall symmetry of the crystal, that is, the position of the molecules in the unit cell, but information on molecular orientation cannot be derived due to extensive disorder. MD simulations have been successfully employed to identify the most likely orientations and intermolecular contacts, although this information cannot be directly input in the 
refinement, because the average unit cell obtained from the simulated structure is considerably more detailed than what can be determined from the experimentally-measured electron density. Rather than simply averaging all positions, it should be possible to model an arrangement of ${ }^{t} \mathrm{BA}$ molecules in which the most favourable intermolecular interactions are present, which could be a better match to the experimental data. For this, it would be necessary to extract from the simulated trajectory all possible pairs of molecules and calculate the energy of the interaction between the molecules in each pair. The PIXEL method for energy calculations would be particularly well suited for this problem, as it determines not only the total energy of the interaction, but also the magnitude of the Coulombic, polarisation, dispersion, and repulsion contributions. It is expected in this case that the dispersion and repulsion contributions to the intermolecular contacts might be as important as the Coulombic contribution.

In order to fully understand the water- ${ }^{t} \mathrm{BA}$ system, a complete systematic screening of the crystallisation by means of pressure of mixtures of different composition should be carried out, as in the present work certain mixtures have been crystallised but could not be characterised. The use of powder X-ray diffraction and Raman spectroscopy might contribute towards this end. Adding more information to the phase diagram, in the form of a density curve in this case, would provide a further insight into the formation of the amine water clathrates which could potentially be used to improve computational models. 


\section{References}

[1] G. A. Jeffrey, J. Inclusion Phenom., 1984, 1, 211-222.

[2] R. K. McMullan, G. A. Jeffrey and T. H. Jordan, J. Chem. Phys., 1967, 47, 1229-1234.

[3] G. A. Jeffrey, Inclusion Compounds, Vol. I, Academic Press, 1984, pp. $135-190$.

[4] D. Stäben and D. Mootz, J. Incl. Phenom. Mol., 1995, 22, 145-154.

[5] Ł. Dobrzycki, P. Taraszewska, R. Boese, M. K. Cyrański and S. A. Cirkel, Angew. Chem. Int. Ed., 2015, 54, 10138-10144.

[6] S. C. Abrahams and W. N. Lipscomb, Acta Cryst., 1952, 5, 93-99.

[7] J. C. W. Folmer, R. L. Withers, T. R. Welberry and J. D. Martin, Phys. Rev. B, 2008, 77, 1-10.

[8] R. K. McMullan, T. H. Jordan and G. A. Jeffrey, J. Chem. Phys., 1967, 47, 1218-1222.

[9] L. Palatinus and G. Chapuis, J. Appl. Cryst., 2007, 40, 786-790.

[10] G. M. Sheldrick, Acta Cryst., 2015, C71, 3-8.

[11] C. B. Hübschle, G. M. Sheldrick and B. Dittrich, J. Appl. Cryst., 2011, 44, 1281-1284.

[12] Bruker-AXS, APEX2, Bruker-AXS, Madison, Wisconsin, USA, 2007.

[13] G. M. Sheldrick, TWINABS v2008-1, Bruker-AXS, Madison, Wisconsin, USA, 2008.

[14] R. H. Blessing, Cryst. Rev., 1987, 1, 3-58.

[15] G. M. Sheldrick, SADABS v2008-1, Bruker-AXS, Madison, Wisconsin, USA, 2008.

[16] G. M. Sheldrick, Acta Cryst., 2015, 71, 3-8.

[17] P. W. Betteridge, J. R. Carruthers, R. I. Cooper, K. Prout and D. J. Watkin, J. Appl. Cryst., 2003, 36, 1487-1487.

[18] L. Schröder, D. J. Watkin, A. Cousson, R. I. Cooper and W. Paulus, J. Appl. Cryst., 2004, 37, 545-550.

[19] C. F. Macrae, I. J. Bruno, J. A. Chisholm, P. R. Edgington, P. McCabe, E. Pidcock, L. Rodriguez-Monge, R. Taylor, J. van de Streek and P. A. Wood, J. Appl. Cryst., 2008, 41, 466-470. 
[20] M. J. Abraham, T. Murtola, R. Schulz, S. Páll, J. C. Smith, B. Hess and E. Lindah, SoftwareX, 2015, 1-2, 19-25.

[21] N. Schmid, A. P. Eichenberger, A. Choutko, S. Riniker, M. Winger, A. E. Mark and W. F. Van Gunsteren, Eur. Biophys. J., 2011, 40, 843-856.

[22] W. L. Jorgensen, D. S. Maxwell and J. Tirado-Rives, J. Am. Chem. Soc., 1996, 118, 11225-11236.

[23] A. Nemkevich, H. B. Bürgi, M. A. Spackman and B. Corry, Phys. Chem. Chem. Phys, 2010, 12, 14916-14929.

[24] C. E. S. Bernardes and A. Joseph, J. Phys. Chem. A, 2015, 119, 3023-3034.

[25] M. J. Frisch, G. W. Trucks, H. B. Schlegel, G. E. Scuseria, M. A. Robb, J. R. Cheeseman, G. Scalmani, V. Barone, B. Mennucci, G. A. Petersson, H. Nakatsuji, M. Caricato, X. Li, H. P. Hratchian, A. F. Izmaylov, J. Bloino, G. Zheng, J. L. Sonnenberg, M. Hada, M. Ehara, K. Toyota, R. Fukuda, J. Hasegawa, M. Ishida, T. Nakajima, Y. Honda, O. Kitao, H. Nakai, T. Vreven, J. A. Montgomery, Jr., J. E. Peralta, F. Ogliaro, M. Bearpark, J. J. Heyd, E. Brothers, K. N. Kudin, V. N. Staroverov, R. Kobayashi, J. Normand, K. Raghavachari, A. Rendell, J. C. Burant, S. S. Iyengar, J. Tomasi, M. Cossi, N. Rega, J. M. Millam, M. Klene, J. E. Knox, J. B. Cross, V. Bakken, C. Adamo, J. Jaramillo, R. Gomperts, R. E. Stratmann, O. Yazyev, A. J. Austin, R. Cammi, C. Pomelli, J. W. Ochterski, R. L. Martin, K. Morokuma, V. G. Zakrzewski, G. A. Voth, P. Salvador, J. J. Dannenberg, S. Dapprich, A. D. Daniels, O. Farkas, J. B. Foresman, J. V. Ortiz, J. Cioslowski and D. J. Fox, GAUSSIAN09 revision A.02, Gaussian Inc., Wallingford CT, 2009.

[26] J. D. Hunter, Comput. Sci. Eng., 2007, 9, 99-104.

[27] G. M. Sheldrick, Acta cryst., 2008, A64, 112-22.

[28] T. Steiner, Acta Cryst., 2002, E41, 49-76.

[29] A. L. Kohl and R. B. Nielsen, Gas Purification, Gulf Publishing Company, 1997.

[30] D. Mootz and D. Stäben, Z. Naturforsch. B, 1993, 48, 1325-1330.

[31] M. Wojdyr, J. Appl. Cryst., 2010, 43, 1126-1128. 
[32] K. Röttger, A. Endriss, J. Ihringer, S. Doyle and W. F. Kuhs, Acta Cryst., 1994, B50, 644-648.

[33] R. E. Gagnon, H. Kiefte, M. J. Clouter and E. Whalley, J. Chem. Phys., 1988, 89, 4522-4528.

[34] T. Strässle, S. Klotz, J. S. Loveday and M. Braden, J. Phys. Condens. Matter, 2005, 17, S3029-S3033.

[35] A. Klapproth, E. Goreshnik, D. Staykova, H. Klein and W. F. Kuhs, Can. J. Phys., 2003, 81, 503-518. 

Appendices 


\section{A List of structural sets}

The following list contains all structural sets extracted from the CSD in which at least one determination has been recorded at high pressure. Reference code words and numbers have been separated to avoid unnecessary repetitions. The reference code $N N N N N N 00$ stands for $N N N N N N$; the reference code number 00 is given only to avoid confusion.

Structural set 1

HP BOLDIP 2223242526

AP BOLDIP 00101112131415161718 192021

Structural set 2

HP CLMETH 01020304

AP CLMETH 00

Structural set 3

HP FOXNUB 1112131415161718

AP FOXNuB 00010203040506070809 10

Structural set 4

HP MBRMET 1112131415

AP MBRMET 10

Structural set 5

HP NALCYS 19202122232425262728

AP NALCYS 00010210111213141516 1718

Structural set 6

HP ABUlit 0304050607

AP ABUlit 000102

Structural set 7

HP ABUMit 01

AP ABUMIT 00

Structural set 8

HP ABUMOZ 01

AP ABUMOZ 00

Structural set 9

HP ACETAC 0809

AP ACETAC 0001020307
Structural set 10

HP ACRLAC 0304

AP ACRLAC 000102

Structural set 11

HP ADAMAN 05

AP ADAMAN 00010203060708

Structural set 12

HP ADMNTB 02

AP ADMNTB 00010304

Structural set 13

HP AFUDEL 020304050607080910

AP AFUDEL 00011112

structural set 14

HP AWIHOE 1112

AP AWIHOE 00010203040506070809

$$
10
$$

Structural set 15

HP BAZGOY 05

AP BAZGOY 000106

structural set 16

HP BEDMIG 131415161718

AP BEDMIG 00010203040506070809 101112

Structural set 17

HP BENZEN 080910111213151617

AP BENZEN 00010218

\section{Structural set 18}

HP BOQCUF 1213

AP BOQCUF 00010203040506070809 1011 
Structural set 19

HP BOQQUT 030405060708

AP BOQQUT 000102

Structural set 20

HP BROFRM 0506

AP BROFRM 00

Structural set 21

HP BUQGUQ 0203

AP BUQGUQ 0001

Structural set 22

HP BZDMAZ 05060708091011121314 15161718192021222324 25262728

AP BZDMAZ 0001020304

Structural set 23

HP CARZIF 03040506070809

AP CARZIF 000102

Structural set 24

HP CILHIO 0708

AP CILHIO 00030405060910121314 15161719202122232426 27283132353637384041 424344

DATREV 01

Structural set 25

HP CLFORM 0102

AP CLFORM 0003

Structural set $\mathbf{2 6}$

HP CLPHOL 14

AP CLPHOL 0210111213

Structural set 27

HP COKCEL 00

Structural set 28

HP CTMTNA 010206

AP CTMTnA 00030405070809

Structural set 29

HP CUKCIU 03

AP CUKCIU 000102
Structural set 30

HP DANTEN 05060708091011

AP DANTEN 00010203041213141516

Structural set 31

HP YISBIM 0001

Structural set 32

HP DBRMET 11

AP DBRMET 10

Structural set 33

HP DCLBEN 10

AP DCLBEN 00010203040506070809 11

Structural set 34

HP DCLMET 1112

AP DCLMET 10

Structural set 35

HP DECWAJ 02060708091011121314

AP DECWAJ 0001030405

Structural set 36

HP DIMETH 11131415

AP DIMETH 0102030405061012

Structural set 37

HP DLALNI 09101112131415161718 192021

AP DLALNI 000102030405060708

Structural set 38

HP DLSERN 19202122232425262728 2930

AP DLSERN $\quad 00021112131415161718$ 313233

Structural set 39

HP DMETSO 040506

AP DMETSO 0001

Structural set 40

HP DOKJAP 02

AP DOKJAP 0001

Structural set 41

HP EFUMAU 03

AP EFUmau 000102 
Structural set 42

HP ETANOL 01

AP ETANOL 00

Structural set 43

HP ETEYAE 00

Structural set 44

HP ETEYEI 00

Structural set 45

HP ETEYIM 00

Structural set 46

HP FACRIK 020304

AP FACRIK 00010506070809

Structural set 47

HP FIGYID 01

AP FIGYID 00

Structural set 48

HP FOHCEK 00010203

AP FOHCEK 04

Structural set 49

HP FORMAC 04

AP FORMAC 0001

Structural set $\mathbf{5 0}$

HP FORMAM 0304

AP FORMAM 000102

Structural set 51

HP GEYREH 02

AP GEYREH 0001

Structural set 52

HP GLUTAM 040506070809

AP GLUTAM 00010203

Structural set 53

$\begin{array}{lll}\text { HP } & \text { GLutas } & 030405 \\ \text { AP } & \text { GLutas } & 0206\end{array}$
Structural set 54

HP GLYCIN $\quad 35363738394041424344$ 45464748495051525354 55565758596061626364 65666768727374757677 7879

AP GLYCIN $\quad 00010203040506070809$ 10111516171819202122 23242526272829303132 33346970718081828384 85868788899091929394 9596979899 DOLBIR $\quad 00010203040506$

Structural set $\mathbf{5 5}$

HP GOFPUN 000102

Structural set 56

HP GUHHAS 010203

AP GUHHAS 00

Structural set $\mathbf{5 7}$

HP Gummuw 040506070809

AP Gummuw 000102031011

Structural set $\mathbf{5 8}$

HP GHEGHUX 00

Structural set 59

HP HIXFIX 00

AP HIXFIX 01020305

Structural set 60

HP IMAzol 15161718192021222324 2526272930313233

AP IMAZOL 010203040506101314

Structural set 61

HP IODOFO 0304

AP IODOFO 000205

Structural set $\mathbf{6 2}$

HP IZOXOL $\quad 000102030405060708$

AP IZOXOL 09 
Structural set 63

HP KECYBU 0708

AP KECYBU 00020305061112131415 161718

Structural set 64

HP KETVEK 03

AP KETVEK 000102

Structural set 65

HP LALNIN 252627282930313233

AP LALNIN

00010312131415161718

192022232452535455

Structural set 66

HP LCYSTI 16171819

AP LCYSTI 101112131415

Structural set 67

HP LCYSTN 24252627

AP LCYSTN 02122122232829303132 33343536

Structural set 68

HP LENBOV 010203

AP LENBOV 04

Structural set 69

HP LETGIA 07080910

AP LETGIA 00010203040506

Structural set 70

HP MCBENZ 00

AP MCBENZ 0102

Structural set 71

HP MECHLF 131415

AP MECHLF 020304101112

Structural set 72

HP MESEFV 050611

AP MESEFV 00041314

Structural set 73

HP MIGPAU 010304050607

AP MIGPAU 0002

Structural set 74

HP MIMETH 121314

AP Mimeth 10
Structural set 75

HP NAGHOT 000203

AP NAGHOT 01

Structural set 76

HP NAPHTA 1219202122

AP NAPHTA 00020405060708091011

15161718232425262728

29303132333435363738

Structural set 77

HP NEBGUX 0102030405

Structural set 78

HP NIMRIK 0102030405

AP NIMRIK 00060708

Structural set 79

HP NIRJAA 02030405

AP NIRJAA 0001

Structural set 80

HP NIRJEE 020304050607

AP NIRJEE 0001

Structural set 81

HP NIRJII 020304050607

AP NIRJII 0001

Structural set 82

HP NTROMA 02030405060708091112

AP NTROMA 0001

Structural set 83

HP NUXJUM 020304

AP NUXJUM 0001

Structural set 84

HP OCRSOL 02

AP OCRSOL 0001

Structural set 85

HP OCUJOM 0002

AP ocuJom 01

QIFNIE 00

Structural set 86

HP OHIJAR 0001020304

Structural set 87

HP OMISIM 18 
Structural set 88

HP POGJOM 000102

Structural set 89

HP POHYIV 00

AP POHYIV 01

Structural set 90

HP PRONAC 0203

AP PRONAC 0001

Structural set 91

HP PYRDNO 1718192021

AP PYRDNO 101116

Structural set 92

HP PYRENE 0809

AP PYRENE 00010203040507

Structural set 93

HP PYRZOL 28293031323334353637 3940

AP PYRZOL 00010204050607080910 11121314151617181920 21232425262738

Structural set 94

HP QAMTUU 01

AP QAMTUU 00

Structural set 95

HP QAMVEG 01

AP QAMVEG 00

Structural set 96

HP QAMVEH 0102

AP QAMVEH 00

Structural set 97

HP QESGOL 0001

Structural set 98

HP QOSCUX 00

Structural set 99

HP QQQAUG 1011121314151617

AP QQQAUG 00010203040506070809 18
Structural set 100

HP QQQBNG 02

AP QQQBNG 0001

Structural set 101

HP QQQCIV 060708

AP QQQCIV 0001030405

Structural set 102

HP RITNOY 0001

AP RITNOY 02

Structural set 103

HP RIVDOQ 0203

AP RIVDOQ 0001

Structural set 104

HP ROMJuz 010304

AP ROMJuz 0002

Structural set 105

HP SACBAA 0001

Structural set 106

HP SALMID 0304050607

AP SALMID 000102

Structural set 107

HP SALOXM 040506070809

AP SALOXM 0001020310

Structural set 108

HP SUCROS 18192021222324252627 28293031323334353637 383940

AP SUCROs 00010304081112131415 1617

Structural set 109

HP SUVGEW 00

Structural set 110

HP SUVGIA 00010203

Structural set 111

HP TAURIN 07

AP TAURIN 01040506080910

Structural set 112

HP TIVWOL 00

AP TIVWOL 01 
Structural set 113

HP Uнuтон 00

Structural set 114

HP UREAXX 24

AP UREAXX 00010203040607080910

1112131415222325

Structural set 115

HP UTIXAX 0102

AP UTIXAX 00

Structural set 116

HP VOBJEB 0102

AP VOBJEB 00

Structural set 117

HP VOFVAN 2223242526

AP VOFVAN 00102021

Structural set 118

HP vuhtoi 000102

Structural set 119

HP VUHVEA 0001

Structural set 120

HP WANMUU 0102

AP WANMUU 00

Structural set 121

HP XAJMUS 00

Structural set 122

HP XAXCOQ 0001

AP XAXCOQ 0203

Structural set 123

HP XUDVOH 05

AP XUDVOH 0001020304

Structural set 124

HP YARZUN 010203

AP YARZUN 00

Structural set 125

HP YIHHON 030405060708091011

AP YIHHON 00021213141516171819 20

Structural set 126

HP YIQGAH 00010203
Structural set 127

HP YOLDAF 02030001

Structural set 128

HP yomXut 04050607

AP YOMXUT 03

Structural set 129

HP ZEFJAX 0001

Structural set 130

HP ZEFPAD 00010203

Structural set 131

HP ZEFPuX 0001

Structural set 132

HP ZZZPRO 02

AP ZZZPRO 000105060708

Structural set 133

HP Citsop 020304050607

AP CITSOP 0001

Structural set 134

HP BALNIN 030405

AP BALNIN 000102

Structural set 135

HP CERKIU 02

AP CERKIU 0001

Structural set 136

HP IBPRAC $\quad 05070809101112131415$

AP IBPRAC $\quad 00010203040616171819$

Structural set 137

HP KOWYEA 03040506070809101112 1314

AP KOWYEA 000102

Structural set 138

HP KUBMAX 0203

AP KUBMAX 0001

Structural set 139

HP KUDMON 0102

AP KUDMON 00 
Set anhydrate-hydrate 1

Structural set 140

HP BISMEV 040607080910

AP BISMEV 0001020305111213

Structural set 141

HP LIFNOE 00

Structural set 142

AP YAKWAJ 0001

Set anhydrate-hydrate 2

Structural set 143

HP BOBVIY 04050607

AP BOBVIY 00010203080910111213 141516

Structural set 144

HP EVUQAO 0001

Set anhydrate-hydrate 3

Structural set 145

HP CYSTEA 00

Structural set 146

HP CYSTAC 030405060708

AP CYSTAC 000102

Set anhydrate-hydrate 4

Structural set 147

HP EJIQEU 0102

AP EJIQEU 00

Structural set 148

AP AMTETZ 0001

Set anhydrate-hydrate 5

Structural set 149

HP ETDIAM 14151617181920

AP ETDIAM 01101213

Structural set 150

AP sowsus 00

Structural set 151

AP GIXDAS 00
Set anhydrate-hydrate 6

Structural set 152

HP GLYGLY 15161718

AP GLYGLY 00010203040506070813 14192021222324252628

Structural set 153

AP POTPET 00010203

Set anhydrate-hydrate 7

Structural set 154

HP HXACAN 09101112

AP HXACAN 00010203040506070813 14151617181920212223 24252627282930313233 34

Structural set 155

AP HUMJEE 00

Structural set 156

HP WAFNAT 00

Structural set 157

AP XOMWOL 00

Set anhydrate-hydrate 8

Structural set 158

AP VENLUW 00

Structural set 159

HP IFIZIG 0203040506070809

AP IFIZIG 0001

Set anhydrate-hydrate 9

Structural set 160

AP NELPUP 00

Structural set 161

HP IMEGIR 010203040506070809

AP IMEGIR 00 
Set anhydrate-hydrate 10

Structural set 162

HP WeMweq 0203040506

AP WEMWEQ 00010708091011121314 15

Structural set 163

HP KICCOO 03040506070809101112

AP $\mathrm{KICCOO} \quad 000102$

Set anhydrate-hydrate 11

Structural set 164

HP LSERIN 11121314151621264142 43444546474849

AP LSERIN $\quad 01020310171819202728$

Structural set 165

HP LSERMH 1112131516

AP LSERMH 101819

Set anhydrate-hydrate 12

Structural set 166

AP OXALAC 00010203040506070809 1011

Structural set 167

HP OXACDH 3637

AP OXACDH $\quad 00010304060709111213$ 14151617181921222324 25263539

Set anhydrate-hydrate 13

Structural set 168

HP PASWUD 00

Structural set 169

AP PASXAK 0001

Structural set 170

HP PASXIS 010203

AP PASXIS 00

Set anhydrate-hydrate 14

Structural set 171

HP PHENOL 11

AP PHENOL 01020310

Structural set 172

AP PHOLHH 00
Set anhydrate-hydrate 15

Structural set 173

HP PIWXEY 0203040506

AP PIWXEY 0001

Structural set 174

AP LAJGUA 00

Set anhydrate-hydrate 16

Structural set 175

HP PUBMUU 23

AP PUBMUU 00010203040506070809 10111213141516171819 202122

Structural set 176

AP PUBMII 0001

Structural set 177

AP FEFRIS 00

Set anhydrate-hydrate 17

Structural set 178

HP PYRDNA 0306

AP PYRDNA 0001

Structural set $\mathbf{1 7 9}$

AP PYRTHA 0001

Set anhydrate-hydrate 18

Structural set 180

HP THIOUR 171819

AP THIOUR 00010203040506080910 11121314

Structural set 181

AP NEXSUG 0001

Structural set 182

AP NEXSIU 0001

Set anhydrate-hydrate 19

Structural set 183

HP VATSAK 01

AP VATSAK 000203

Structural set 184

AP LEBJUX 00

Structural set 185

AP LEBKEI 00 
Set anhydrate-hydrate 20

Structural set 186

AP QIMKIG 000102030405

Structural set 187

AP QIMKOM 000102

Structural set 188

HP YUZTET 00

Set anhydrate-hydrate 21

Structural set 189

AP gambut 0102030410

Structural set 190

HP ZIPWOM 00

AP ZIPWOM 01
Set anhydrate-hydrate 22

Structural set 191

HP LOWFUA 00

HP GLUCSA 11121314151617181920 21222324

AP Gambut 0002031025

Structural set 192

AP GLUCMH 0001

Set anhydrate-hydrate 23

Structural set 193

HP NEDMIS 020304050607

AP NEDMIS 0001

Structural set 194

AP TOKYIC 00 


\section{B Compressibility of lattice water}

$V_{\text {cell }}, V_{H}$, and $V_{H, \text { void }}$ for all hydrates with at least an HP determination.

\begin{tabular}{|c|c|c|c|c|c|c|c|c|c|c|c|c|c|}
\hline \multicolumn{14}{|c|}{ IN-SITU FORMED HYDRATES } \\
\hline 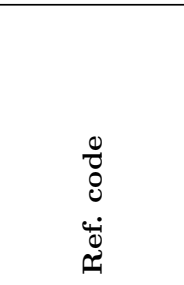 & $\begin{array}{l}0 \\
\text { D } \\
\frac{\sigma}{6} \\
\frac{1}{n}\end{array}$ & $\underset{E}{E}$ & $\underbrace{\overparen{\sigma}}_{R}$ & $\mathbf{N}$ & 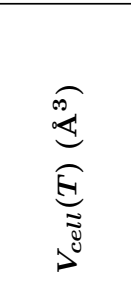 & 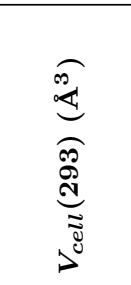 & 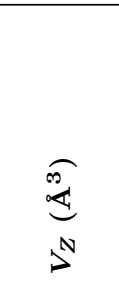 & Z & 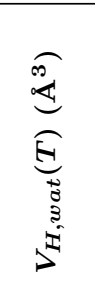 & 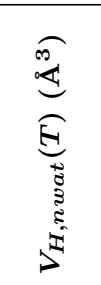 & 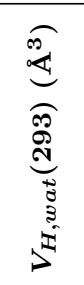 & 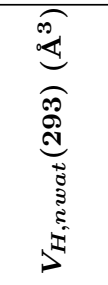 & 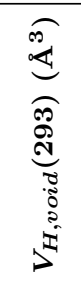 \\
\hline \multicolumn{14}{|c|}{ Piracetam } \\
\hline YAKWAJ & $1 \mathrm{H}$ & 150 & 0.0001 & 2 & 396.238 & 401.621 & 200.810 & 1 & 22.78 & 168.91 & 23.09 & 171.20 & 6.52 \\
\hline YAKWAJ01 & $1 \mathrm{H}$ & 100 & 0.0001 & 2 & 393.225 & 400.435 & 200.217 & 1 & 22.60 & 167.60 & 23.01 & 170.67 & 6.53 \\
\hline \multirow[t]{2}{*}{ LIFNOE } & $2 \mathrm{H}$ & 293 & 0.60 & 2 & 414.974 & 414.974 & 207.487 & 2 & 22.32 & 157.33 & 22.32 & 157.33 & 7.67 \\
\hline & & & & & & & & & 19.62 & & 20.17 & & \\
\hline \multicolumn{14}{|c|}{ Paracetamol } \\
\hline HUMJEE & $1 \mathrm{H}$ & 150 & 0.0001 & 4 & 804.177 & 815.102 & 203.775 & 1 & 21.99 & 172.04 & 22.29 & 174.38 & 7.11 \\
\hline \multirow[t]{3}{*}{ XOMWOL } & $3 \mathrm{H}$ & 150 & 0.0001 & 8 & 2089.640 & 2118.028 & 264.753 & 3 & 20.51 & 185.56 & 20.79 & 188.08 & 10.63 \\
\hline & & & & & & & & & 22.09 & & 22.70 & & \\
\hline & & & & & & & & & 21.94 & & 22.55 & & \\
\hline \multicolumn{14}{|c|}{ Gaba } \\
\hline ZIPWOM01 & $1 \mathrm{H}$ & 150 & 0.0001 & 8 & 1169.055 & 1184.937 & 148.117 & 1 & 20.33 & 120.06 & 20.61 & 121.69 & 5.82 \\
\hline ZIPWOM & $1 \mathrm{H}$ & 296 & 0.44 & 8 & 1151.612 & 1151.284 & 143.910 & 1 & 20.07 & 118.19 & 20.06 & 118.16 & 5.69 \\
\hline \multicolumn{14}{|c|}{ Gabapentin } \\
\hline QIMKOM & $1 \mathrm{H} \mathrm{I}$ & 153 & 0.0001 & 4 & 1025.190 & 1038.825 & 259.706 & 1 & 21.20 & 227.99 & 21.48 & 231.02 & 7.20 \\
\hline QIMKOM01 & $1 \mathrm{H} \mathrm{I}$ & 293 & 0.0001 & 4 & 1043.111 & 1043.111 & 260.778 & 1 & 21.92 & 231.76 & 21.92 & 231.76 & 7.10 \\
\hline QIMKOM02 & $1 \mathrm{H} \mathrm{II}$ & 293 & 0.0001 & 8 & 2074.345 & 2074.345 & 259.293 & 1 & 21.77 & 230.45 & 21.77 & 230.45 & 7.07 \\
\hline \multirow[t]{7}{*}{ YUZTET } & $7 \mathrm{H}$ & 293 & 0.90 & 2 & 737.485 & 737.485 & 368.743 & 7 & 21.01 & 207.56 & 21.01 & 207.56 & 14.32 \\
\hline & & & & & & & & & 21.53 & & 22.13 & & \\
\hline & & & & & & & & & 20.05 & & 20.61 & & \\
\hline & & & & & & & & & 19.68 & & 20.23 & & \\
\hline & & & & & & & & & 20.22 & & 20.78 & & \\
\hline & & & & & & & & & 20.96 & & 21.54 & & \\
\hline & & & & & & & & & 20.00 & & 20.56 & & \\
\hline \multicolumn{14}{|c|}{ dabcoHI } \\
\hline EVUQAO & $1 \mathrm{H} \alpha$ & 296 & 0.5 & 40 & 9794.255 & 9791.464 & 244.787 & 1 & 22.48 & 212.15 & 22.47 & 212.09 & 10.22 \\
\hline EVUQAO01 & $1 \mathrm{H} \beta$ & 296 & 1.4 & 4 & 856.679 & 856.435 & 214.109 & 1 & 19.06 & 185.91 & 19.05 & 185.86 & 9.20 \\
\hline \multicolumn{14}{|c|}{ Thiourea } \\
\hline NEXSUG01 & $0.67 \mathrm{H}$ & 296 & 0.70 & 12 & 1154.473 & 1154.144 & 96.179 & 0.67 & 20.93 & 77.88 & 20.92 & 77.86 & 4.30 \\
\hline NEXSUG & $0.67 \mathrm{H}$ & 296 & 0.95 & 12 & 1132.561 & 1132.238 & 94.353 & 0.67 & 20.46 & 76.61 & 20.45 & 76.59 & 4.06 \\
\hline NEXSIU01 & $1 \mathrm{H}$ & 296 & 0.60 & 4 & 425.817 & 425.696 & 106.424 & 1 & 20.92 & 80.06 & 20.91 & 80.04 & 5.47 \\
\hline NEXSIU & $1 \mathrm{H}$ & 296 & 1.20 & 4 & 411.966 & 411.849 & 102.962 & 1 & 20.11 & 77.51 & 20.10 & 77.49 & 5.37 \\
\hline \multicolumn{14}{|c|}{ dabco2HBr } \\
\hline PASXAK & $0.5 \mathrm{H} \alpha$ & 296 & 0.0001 & 8 & 2030.353 & 2029.774 & 253.722 & 0.5 & 22.84 & 231.47 & 22.83 & 231.40 & 10.90 \\
\hline PASXIS & $1 \mathrm{H} \alpha$ & 296 & 0.0001 & 8 & 2080.335 & 2079.742 & 259.968 & 1 & 23.32 & 224.54 & 23.31 & 224.48 & 12.18 \\
\hline PASXIS02 & $1 \mathrm{H} \gamma$ & 296 & 1.50 & 2 & 450.385 & 450.257 & 225.128 & 1 & 21.12 & 193.32 & 21.11 & 193.26 & 10.75 \\
\hline PASXIS03 & $1 \mathrm{H} \delta$ & 296 & 0.78 & 2 & 490.151 & 490.011 & 245.006 & 1 & 21.61 & 212.36 & 21.60 & 212.30 & 11.10 \\
\hline
\end{tabular}




\section{COMPRESSED HYDRATES}

\begin{tabular}{|c|c|c|c|c|c|c|c|c|c|c|c|c|c|}
\hline 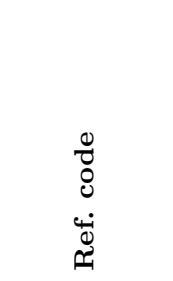 & 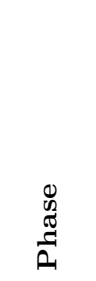 & $\underset{E}{E}$ & 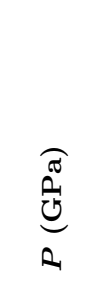 & $\mathbf{N}$ & 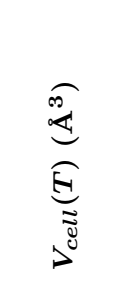 & 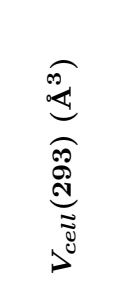 & $\underset{i^{N}}{\stackrel{\infty}{0^{2}}}$ & Z & 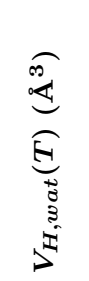 & 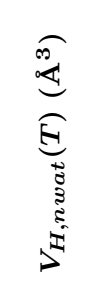 & 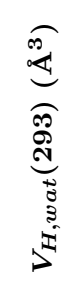 & 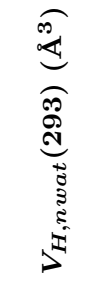 & 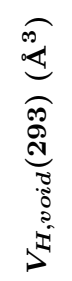 \\
\hline \multicolumn{14}{|c|}{ Cysteic acid } \\
\hline CYSTAC & $1 \mathrm{H}$ & 295 & 0.0001 & 4 & 699.199 & 699.066 & 174.767 & 1 & 21.81 & 147.11 & 21.81 & 147.08 & 5.88 \\
\hline CYSTAC01 & $1 \mathrm{H}$ & 295 & 0.0001 & 4 & 699.199 & 699.066 & 174.767 & 1 & 21.90 & 146.83 & 21.90 & 146.80 & 6.07 \\
\hline CYSTAC02 & $1 \mathrm{H}$ & 293 & 0.0001 & 4 & 698.386 & 698.386 & 174.597 & 1 & 21.83 & 146.75 & 21.83 & 146.75 & 6.02 \\
\hline CYSTAC03 & $1 \mathrm{H}$ & 293 & 0.20 & 4 & 693.060 & 693.060 & 173.265 & 1 & 21.79 & 145.49 & 21.79 & 145.49 & 5.98 \\
\hline CYSTAC04 & $1 \mathrm{H}$ & 293 & 1.20 & 4 & 665.239 & 665.239 & 166.310 & 1 & 20.43 & 139.98 & 20.43 & 139.98 & 5.90 \\
\hline CYSTAC05 & $1 \mathrm{H}$ & 293 & 2.80 & 4 & 633.205 & 633.205 & 158.301 & 1 & 18.93 & 133.58 & 18.93 & 133.58 & 5.79 \\
\hline CYSTAC06 & $1 \mathrm{H}$ & 293 & 4.50 & 4 & 611.870 & 611.870 & 152.968 & 1 & 18.05 & 129.18 & 18.05 & 129.18 & 5.74 \\
\hline \multicolumn{14}{|c|}{ Phenyl alanine } \\
\hline IFIZIG & $1 \mathrm{H} \mathrm{I}$ & 295 & 0.0001 & 2 & 576.668 & 576.558 & 288.279 & 1 & 24.25 & 256.13 & 24.25 & 256.08 & 7.95 \\
\hline IFIZIG01 & $1 \mathrm{H} \mathrm{I}$ & 293 & 0.0001 & 2 & 578.216 & 578.216 & 289.108 & 1 & 24.38 & 256.85 & 24.38 & 256.85 & 7.88 \\
\hline IFIZIG02 & $1 \mathrm{H} \mathrm{I}$ & 293 & 0.20 & 2 & 571.119 & 571.119 & 285.560 & 1 & 23.83 & 253.89 & 23.83 & 253.89 & 7.84 \\
\hline IFIZIG03 & $1 \mathrm{H} \mathrm{I}$ & 293 & 1.00 & 2 & 545.135 & 545.135 & 272.568 & 1 & 22.39 & 242.42 & 22.39 & 242.42 & 7.76 \\
\hline IFIZIG04 & $1 \mathrm{H} \mathrm{II}$ & 293 & 2.50 & 4 & 974.611 & 974.611 & 243.653 & 1 & 18.14 & 218.63 & 18.14 & 218.63 & 6.88 \\
\hline IFIZIG05 & $1 \mathrm{H} \mathrm{II}$ & 293 & 4.00 & 4 & 936.253 & 936.253 & 234.063 & 1 & 17.11 & 210.14 & 17.11 & 210.14 & 6.81 \\
\hline \multicolumn{14}{|c|}{ Alaninium oxalate } \\
\hline IMEGIR & $1 \mathrm{H}$ & 300 & 0.0001 & 4 & 860.043 & 859.471 & 214.868 & 1 & 21.00 & 185.28 & 20.99 & 185.16 & 8.72 \\
\hline IMEGIR01 & $1 \mathrm{H}$ & 293 & 0.30 & 4 & 839.975 & 839.975 & 209.994 & 1 & 20.46 & 180.92 & 20.46 & 180.92 & 8.61 \\
\hline IMEGIR02 & $1 \mathrm{H}$ & 293 & 0.50 & 4 & 830.109 & 830.109 & 207.527 & 1 & 20.11 & 178.83 & 20.11 & 178.83 & 8.59 \\
\hline IMEGIR03 & $1 \mathrm{H}$ & 293 & 1.10 & 4 & 805.899 & 805.899 & 201.475 & 1 & 19.26 & 173.77 & 19.26 & 173.77 & 8.44 \\
\hline IMEGIR04 & $1 \mathrm{H}$ & 293 & 1.50 & 4 & 791.348 & 791.348 & 197.837 & 1 & 18.83 & 170.60 & 18.83 & 170.60 & 8.41 \\
\hline IMEGIR05 & $1 \mathrm{H}$ & 293 & 2.40 & 4 & 745.952 & 745.952 & 186.488 & 1 & 17.26 & 161.12 & 17.26 & 161.12 & 8.11 \\
\hline IMEGIR06 & $1 \mathrm{H}$ & 293 & 3.10 & 4 & 725.179 & 725.179 & 181.295 & 1 & 16.79 & 156.48 & 16.79 & 156.48 & 8.02 \\
\hline IMEGIR07 & $1 \mathrm{H}$ & 293 & 3.60 & 4 & 713.457 & 713.457 & 178.364 & 1 & 16.67 & 153.78 & 16.67 & 153.78 & 7.91 \\
\hline IMEGIR08 & $1 \mathrm{H}$ & 293 & 4.30 & 4 & 700.812 & 700.812 & 175.203 & 1 & 16.26 & 151.06 & 16.26 & 151.06 & 7.88 \\
\hline \multicolumn{14}{|c|}{ Betaine } \\
\hline $\mathrm{KICCOO}$ & $1 \mathrm{H}$ & 295 & 0.0001 & 8 & 1428.353 & 1428.082 & 178.510 & 1 & 21.99 & 150.92 & 21.99 & 150.89 & 5.63 \\
\hline KICCOO02 & $1 \mathrm{H}$ & 293 & 0.0001 & 8 & 1424.266 & 1424.266 & 178.033 & 1 & 21.99 & 150.42 & 21.99 & 150.42 & 5.62 \\
\hline KICCOO03 & $1 \mathrm{H}$ & 293 & 0.10 & 8 & 1404.275 & 1404.275 & 175.534 & 1 & 21.57 & 148.37 & 21.57 & 148.37 & 5.59 \\
\hline KICCOO04 & $1 \mathrm{H}$ & 293 & 1.60 & 8 & 1284.940 & 1284.940 & 160.618 & 1 & 19.22 & 135.98 & 19.22 & 135.98 & 5.42 \\
\hline KICCOO05 & $1 \mathrm{H}$ & 293 & 2.90 & 8 & 1227.902 & 1227.902 & 153.488 & 1 & 18.07 & 130.08 & 18.07 & 130.08 & 5.34 \\
\hline KICCOO06 & $1 \mathrm{H}$ & 293 & 4.00 & 8 & 1190.086 & 1190.086 & 148.761 & 1 & 17.35 & 126.13 & 17.35 & 126.13 & 5.28 \\
\hline KICCOO07 & $1 \mathrm{H}$ & 293 & 4.90 & 8 & 1163.267 & 1163.267 & 145.408 & 1 & 16.83 & 123.33 & 16.83 & 123.33 & 5.25 \\
\hline \multicolumn{14}{|c|}{ Serine } \\
\hline LSERMH10 & $1 \mathrm{H}$ & 295 & 0.0001 & 4 & 554.179 & 554.074 & 138.518 & 1 & 23.04 & 109.84 & 23.04 & 109.82 & 5.66 \\
\hline LSERMH18 & $1 \mathrm{H}$ & 97 & 0.0001 & 4 & 542.418 & 552.518 & 138.129 & 1 & 22.26 & 107.74 & 22.67 & 109.75 & 5.71 \\
\hline LSERMH19 & $1 \mathrm{H}$ & 120 & 0.0001 & 4 & 542.049 & 550.958 & 137.739 & 1 & 22.25 & 107.65 & 22.62 & 109.42 & 5.70 \\
\hline LSERMH11 & $1 \mathrm{H}$ & 293 & 1.00 & 4 & 522.922 & 522.922 & 130.731 & 1 & 21.24 & 104.03 & 21.24 & 104.03 & 5.46 \\
\hline LSERMH12 & $1 \mathrm{H}$ & 293 & 2.60 & 4 & 494.272 & 494.272 & 123.568 & 1 & 19.49 & 98.75 & 19.49 & 98.75 & 5.33 \\
\hline LSERMH13 & $1 \mathrm{H}$ & 293 & 3.70 & 4 & 476.027 & 476.027 & 119.007 & 1 & 18.43 & 95.35 & 18.43 & 95.35 & 5.23 \\
\hline LSERMH15 & $1 \mathrm{H}$ & 293 & 4.50 & 4 & 463.213 & 463.213 & 115.803 & 1 & 17.67 & 93.01 & 17.67 & 93.01 & 5.12 \\
\hline
\end{tabular}




\begin{tabular}{|c|c|c|c|c|c|c|c|c|c|c|c|c|c|}
\hline \multirow[b]{2}{*}{ 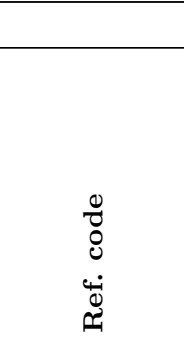 } & \multicolumn{12}{|c|}{ COMPRESSED HYDRATES (continue) } & \multirow[b]{2}{*}{ 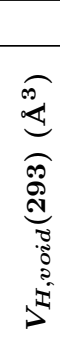 } \\
\hline & \begin{tabular}{l}
0 \\
\multirow{\sigma}{\sigma}{} \\
$\mathcal{L}$
\end{tabular} & $\underset{E}{E}$ & $\underbrace{\stackrel{\sigma}{\sigma}}_{\mathcal{R}}$ & $\mathbf{N}$ & \multicolumn{2}{|r|}{ 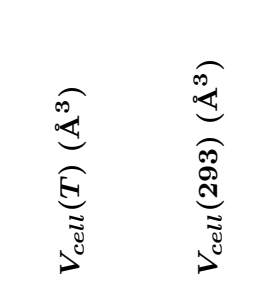 } & $\underset{D^{N}}{\stackrel{m}{0}}$ & \multirow[t]{2}{*}{ Z } & 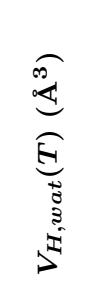 & 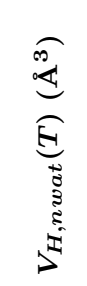 & 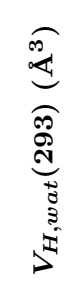 & 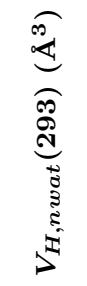 & \\
\hline \multicolumn{13}{|c|}{ Oxalic acid } & \\
\hline OXACDH01 & $2 \mathrm{H}$ & 100 & 0.0001 & 2 & 245.612 & 250.115 & 125.058 & 2 & 20.99 & 73.80 & 21.37 & 75.15 & 6.96 \\
\hline & & & & & & & & & & 20.99 & & 21.57 & \\
\hline \multirow[t]{2}{*}{ OXACDH04 } & $2 \mathrm{H}$ & 295 & 0.0001 & 2 & 255.390 & 255.341 & 127.671 & 2 & 21.94 & 76.66 & 21.94 & 76.65 & 6.54 \\
\hline & & & & & & & & & & 21.94 & & 22.55 & \\
\hline \multirow[t]{2}{*}{ OXACDH06 } & $2 \mathrm{H}$ & 100 & 0.0001 & 2 & 245.135 & 249.630 & 124.815 & 2 & 20.97 & 73.61 & 21.35 & 74.96 & 6.95 \\
\hline & & & & & & & & & & 20.97 & & 21.55 & \\
\hline \multirow[t]{2}{*}{ OXACDH11 } & $2 \mathrm{H}$ & 295 & 0.0001 & 2 & 255.390 & 255.341 & 127.671 & 2 & 21.88 & 76.86 & 21.88 & 79.00 & 4.91 \\
\hline & & & & & & & & & & 21.88 & & 21.88 & \\
\hline \multirow[t]{2}{*}{ OXACDH15 } & $2 \mathrm{H}$ & 100 & 0.0001 & 2 & 245.951 & 250.461 & 125.230 & 2 & 21.02 & 73.90 & 21.41 & 75.25 & 6.96 \\
\hline & & & & & & & & & & 21.02 & & 21.61 & \\
\hline \multirow[t]{2}{*}{ OXACDH16 } & $2 \mathrm{H}$ & 100 & 0.0001 & 2 & 245.951 & 250.461 & 125.230 & 2 & 21.02 & 73.91 & 21.41 & 75.27 & 6.95 \\
\hline & & & & & & & & & & 21.02 & & 21.61 & \\
\hline \multirow[t]{2}{*}{ OXACDH17 } & $2 \mathrm{H}$ & 130 & 0.0001 & 2 & 247.333 & 251.163 & 125.581 & 2 & 21.09 & 74.45 & 21.42 & 75.60 & 6.88 \\
\hline & & & & & & & & & & 21.09 & & 21.68 & \\
\hline \multirow[t]{2}{*}{ OXACDH18 } & $2 \mathrm{H}$ & 130 & 0.0001 & 2 & 247.333 & 251.163 & 125.581 & 2 & 21.09 & 74.45 & 21.42 & 75.60 & 6.88 \\
\hline & & & & & & & & & & 21.09 & & 21.68 & \\
\hline \multirow[t]{2}{*}{ OXACDH19 } & $2 \mathrm{H}$ & 170 & 0.0001 & 2 & 248.134 & 251.033 & 125.517 & 2 & 21.23 & 74.56 & 21.48 & 75.43 & 6.79 \\
\hline & & & & & & & & & & 21.23 & & 21.82 & \\
\hline OXACDH 21 & $2 \mathrm{H}$ & 170 & 0.0001 & 2 & 248.134 & 251.033 & 125.517 & 2 & 21.21 & 74.59 & 21.46 & 75.46 & 6.80 \\
\hline & & & & & & & & & & 21.21 & & 21.80 & \\
\hline OXACDH22 & $2 \mathrm{H}$ & 225 & 0.0001 & 2 & 250.974 & 252.595 & 126.298 & 2 & 21.48 & 75.44 & 21.62 & 75.93 & 6.67 \\
\hline & & & & & & & & & & 21.48 & & 22.08 & \\
\hline OXACDH 23 & $2 \mathrm{H}$ & 225 & 0.0001 & 2 & 250.974 & 252.595 & 126.298 & 2 & 21.47 & 75.47 & 21.61 & 75.96 & 6.66 \\
\hline & & & & & & & & & & 21.47 & & 22.07 & \\
\hline OXACDH 24 & $2 \mathrm{H}$ & 295 & 0.0001 & 2 & 255.386 & 255.337 & 127.669 & 2 & 21.95 & 76.68 & 21.95 & 76.67 & 6.50 \\
\hline & & & & & & & & & & 21.95 & & 22.56 & \\
\hline OXACDH26 & $2 \mathrm{H}$ & 15 & 0.0001 & 2 & 242.683 & 249.092 & 124.546 & 2 & 20.74 & 72.84 & 21.29 & 74.76 & 7.18 \\
\hline & & & & & & & & & & 20.74 & & 21.32 & \\
\hline OXACDH35 & $2 \mathrm{H}$ & 295 & 0.0001 & 2 & 255.035 & 254.987 & 127.493 & 2 & 21.96 & 76.51 & 21.96 & 76.50 & 6.47 \\
\hline & & & & & & & & & & 21.96 & & 22.57 & \\
\hline OXACDH39 & $2 \mathrm{H}$ & 293 & 0.0001 & 2 & 247.270 & 247.270 & 123.635 & 2 & 21.17 & 74.24 & 21.17 & 74.24 & 6.47 \\
\hline & & & & & & & & & & 21.17 & & 21.76 & \\
\hline OXACDH36 & $2 \mathrm{H}$ & 295 & 1.60 & 2 & 226.518 & 226.475 & 113.237 & 2 & 19.11 & 68.28 & 19.11 & 68.27 & 6.22 \\
\hline & & & & & & & & & & 19.11 & & 19.64 & \\
\hline OXACDH37 & $2 \mathrm{H}$ & 295 & 3.60 & 2 & 206.399 & 206.360 & 103.180 & 2 & 17.16 & 62.36 & 17.16 & 62.35 & 6.04 \\
\hline & & & & & & & & & & 17.16 & & 17.64 & \\
\hline
\end{tabular}




\section{Volume-pressure diagrams}

Calculation of $V_{\text {excl }}$ for all hydrates with at least an HP determination.

\begin{tabular}{|c|c|c|c|c|c|c|c|c|c|c|}
\hline \multicolumn{11}{|c|}{ IN SITU-FORMED HYDRATES } \\
\hline 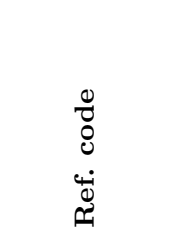 & 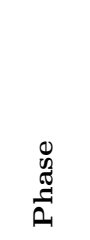 & $\underbrace{E}_{E}$ & 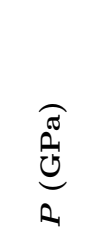 & $\mathbf{N}$ & 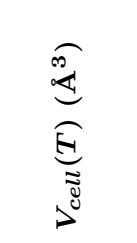 & 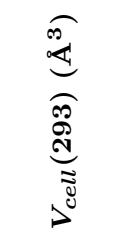 & $\begin{array}{l}\stackrel{\infty}{\infty} \\
\overbrace{}^{N}\end{array}$ & Z & 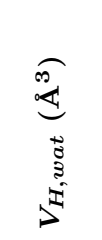 & $\begin{array}{l}\widetilde{\infty} \\
\widetilde{\infty} \\
\widetilde{\tilde{J}} \\
\mathcal{D}^{2}\end{array}$ \\
\hline \multicolumn{11}{|c|}{ Piracetam } \\
\hline BISMEV03 & I & 295 & 0.0001 & 4 & 723.361 & 723.224 & 180.806 & 0 & 0 & 180.806 \\
\hline BISMEV & II & 295 & 0.0001 & 2 & 348.508 & 348.442 & 174.221 & 0 & 0 & 174.221 \\
\hline BISMEV06 & II & 293 & 0.45 & 2 & 334.013 & 334.013 & 167.007 & 0 & 0 & 167.007 \\
\hline BISMEV02 & III & 295 & 0.0001 & 4 & 684.160 & 684.030 & 171.008 & 0 & 0 & 171.008 \\
\hline BISMEV04 & IV & 293 & 0.40 & 4 & 642.199 & 642.199 & 160.550 & 0 & 0 & 160.550 \\
\hline BISMEV07 & $\mathrm{V}$ & 293 & 0.70 & 2 & 325.534 & 325.534 & 162.767 & 0 & 0 & 162.767 \\
\hline BISMEV08 & $\mathrm{V}$ & 293 & 0.90 & 2 & 314.262 & 314.262 & 157.131 & 0 & 0 & 157.131 \\
\hline BISMEV09 & $\mathrm{V}$ & 293 & 2.50 & 2 & 292.826 & 292.826 & 146.413 & 0 & 0 & 146.413 \\
\hline BISMEV10 & $\mathrm{V}$ & 293 & 4.00 & 2 & 280.206 & 280.206 & 140.103 & 0 & 0 & 140.103 \\
\hline YAKWAJ01 & $1 \mathrm{H}$ & 100 & 0.0001 & 2 & 393.225 & 400.435 & 200.217 & 1 & 22.37 & 177.85 \\
\hline LIFNOE & $2 \mathrm{H}$ & 293 & 0.60 & 2 & 414.974 & 414.974 & 207.487 & 2 & 41.86 & 165.62 \\
\hline \multicolumn{11}{|c|}{ Paracetamol } \\
\hline HXACAN01 & $\mathrm{I}$ & 295 & 0.0001 & 4 & 776.272 & 776.125 & 194.031 & 0 & 0 & 194.031 \\
\hline HXACAN09 & I & 295 & 1.00 & 4 & 711.734 & 711.599 & 177.900 & 0 & 0 & 177.900 \\
\hline HXACAN10 & I & 295 & 2.00 & 4 & 672.012 & 671.884 & 167.971 & 0 & 0 & 167.971 \\
\hline HXACAN11 & I & 295 & 3.00 & 4 & 651.428 & 651.304 & 162.826 & 0 & 0 & 162.826 \\
\hline HXACAN12 & I & 295 & 4.00 & 4 & 621.881 & 621.763 & 155.441 & 0 & 0 & 155.441 \\
\hline HXACAN33 & II & 293 & 0.0001 & 8 & 1500.185 & 1500.185 & 187.523 & 0 & 0 & 187.523 \\
\hline HXACAN29 & III & 300 & 0.0001 & 8 & 1501.411 & 1500.413 & 187.552 & 0 & 0 & 187.552 \\
\hline HUMJEE & $1 \mathrm{H}$ & 150 & 0.0001 & 4 & 804.177 & 815.102 & 203.775 & 1 & 22.37 & 181.41 \\
\hline WAFNAT & $2 \mathrm{H}$ & 293 & 1.10 & 4 & 854.296 & 854.296 & 213.574 & 2 & 40.04 & 173.53 \\
\hline XOMWOL & $3 \mathrm{H}$ & 150 & 0.0001 & 8 & 2089.640 & 2118.028 & 264.753 & 3 & 67.10 & 197.65 \\
\hline \multicolumn{11}{|c|}{ GABA } \\
\hline GAMBUT10 & I & 295 & 0.0001 & 4 & 561.147 & 561.040 & 140.260 & 0 & 0 & 140.260 \\
\hline GAMBUT04 & II & 295 & 0.0001 & 16 & 2187.059 & 2186.643 & 136.665 & 0 & 0 & 136.665 \\
\hline ZIPWOM & $1 \mathrm{H}$ & 296 & 0.44 & 8 & 1151.612 & 1151.284 & 143.910 & 1 & 21.27 & 122.64 \\
\hline ZIPWOM01 & $1 \mathrm{H}$ & 150 & 0.0001 & 8 & 1169.055 & 1184.937 & 148.117 & 1 & 22.37 & 125.75 \\
\hline \multicolumn{11}{|c|}{ Gabapentin } \\
\hline QIMKIG01 & II & 293 & 0.0001 & 4 & 918.086 & 918.086 & 229.522 & 0 & 0 & 229.522 \\
\hline QIMKIG05 & III & 293 & 0.0001 & 8 & 1879.865 & 1879.865 & 234.983 & 0 & 0 & 234.983 \\
\hline QIMKIG04 & IV & 293 & 0.0001 & 4 & 933.391 & 933.391 & 233.348 & 0 & 0 & 233.348 \\
\hline QIMKOM01 & $1 \mathrm{H} \mathrm{I}$ & 293 & 0.0001 & 4 & 1043.111 & 1043.111 & 260.778 & 1 & 22.37 & 238.41 \\
\hline QIMKOM02 & $1 \mathrm{H} \mathrm{II}$ & 293 & 0.0001 & 8 & 2074.345 & 2074.345 & 259.293 & 1 & 22.37 & 236.93 \\
\hline YUZTET & $7 \mathrm{H}$ & 293 & 0.90 & 2 & 737.485 & 737.485 & 368.743 & 7 & 142.53 & 226.22 \\
\hline \multicolumn{11}{|c|}{ Parabanic acid } \\
\hline PARBAC02 & I & 295 & 0.0001 & 4 & 441.756 & 441.672 & 110.418 & 0 & 0 & 110.418 \\
\hline BINWEC & $1.5 \mathrm{H}$ & 293 & 0.40 & 8 & 1062.489 & 1062.489 & 132.811 & 1.5 & 31.72 & 101.09 \\
\hline
\end{tabular}




\begin{tabular}{|c|c|c|c|c|c|c|c|c|c|c|}
\hline \multicolumn{11}{|c|}{ IN SITU-FORMED HYDRATES (continue) } \\
\hline 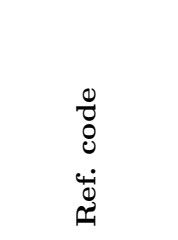 & 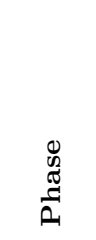 & $\underset{E}{E}$ & $\underbrace{\stackrel{\sigma}{\tilde{C}}}_{A}$ & $\mathbf{N}$ & 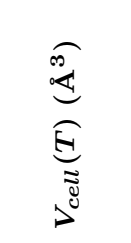 & 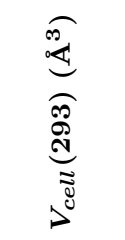 & $\overbrace{i^{N}}^{\stackrel{\infty}{0^{N}}}$ & Z & 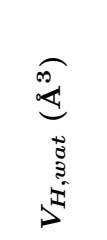 & $\begin{array}{c}\widetilde{m} \\
\widetilde{0} \\
\widetilde{\delta} \\
\nu^{\circ}\end{array}$ \\
\hline \multicolumn{11}{|c|}{ tert-Butylamine } \\
\hline & I & 133 & 0.0001 & 12 & 1694.1 & 1719.9 & 143.3 & 0 & 0 & 143.3 \\
\hline & $0.25 \mathrm{H}$ & 173 & 0.0001 & 32 & 4668.0 & 4721.2 & 147.5 & 0.25 & 5.59 & 141.9 \\
\hline & $1 \mathrm{H}$ & 173 & 0.0001 & 4 & 620.7 & 627.8 & 156.9 & 1 & 22.37 & 134.6 \\
\hline & $7.25 \mathrm{H}$ & 203 & 0.0001 & 32 & 10421.7 & 10510.8 & 328.5 & 7.25 & 162.17 & 166.3 \\
\hline & $7.75 \mathrm{H}$ & 225 & 0.0001 & 16 & 5461.3 & 5496.6 & 343.5 & 7.75 & 173.35 & 170.2 \\
\hline & $9.75 \mathrm{H}$ & 218 & 0.0001 & 16 & 6577.1 & 6624.0 & 414.0 & 9.75 & 218.09 & 195.9 \\
\hline & $11 \mathrm{H}$ & 250 & 0.0001 & 4 & 1795.7 & 1803.0 & 450.8 & 11 & 246.05 & 204.7 \\
\hline & $17 \mathrm{H}$ & 243 & 0.0001 & 8 & 5409.4 & 5435.1 & 679.4 & 17 & 380.25 & 299.1 \\
\hline & II & 293 & 0.78 & 4 & 525.0 & 525.0 & 131.2 & 0 & 0 & 131.2 \\
\hline & $5.65 \mathrm{H}$ & 293 & 0.76 & 32 & 8317.8 & 8317.8 & 259.9 & 5.65 & 116.49 & 143.4 \\
\hline & $5.8 \mathrm{H}$ & 293 & 0.76 & 20 & 5207.1 & 5207.1 & 260.4 & 5.8 & 119.58 & 140.8 \\
\hline \multicolumn{11}{|c|}{ dMBzIm } \\
\hline & $\alpha$ & 296 & 0.11 & 12 & 2469 & 2468 & 206 & 0 & 0 & 206 \\
\hline & $\alpha$ & 296 & 0.22 & 12 & 2443 & 2442 & 204 & 0 & 0 & 204 \\
\hline & $\beta$ & 296 & 0.28 & 12 & 2410 & 2409 & 201 & 0 & 0 & 201 \\
\hline & $\beta$ & 296 & 0.33 & 12 & 2398 & 2397 & 200 & 0 & 0 & 200 \\
\hline & $\beta$ & 296 & 0.64 & 12 & 2333 & 2332 & 194 & 0 & 0 & 194 \\
\hline & $0.5 \mathrm{H}$ & 296 & 0.0001 & 16 & 3274.5 & 3273.6 & 204.6 & 0.5 & 10.82 & 193.8 \\
\hline & $0.5 \mathrm{H}$ & 296 & 0.54 & 16 & 3115 & 3114 & 195 & 0.5 & 10.49 & 184 \\
\hline & $0.5 \mathrm{H}$ & 296 & 0.70 & 16 & 3059 & 3058 & 191 & 0.5 & 10.39 & 181 \\
\hline & $0.5 \mathrm{H}$ & 296 & 1.10 & 16 & 2980.3 & 2979 & 186 & 0.5 & 10.16 & 176 \\
\hline & $0.5 \mathrm{H}$ & 296 & 1.49 & 16 & 2899.1 & 2898.3 & 181.1 & 0.5 & 9.93 & 171.2 \\
\hline \multicolumn{11}{|c|}{ dabcoHI } \\
\hline BOBVIY08 & IV & 400 & 0.0001 & 1 & 220.355 & 218.115 & 218.115 & 0 & 0 & 218.115 \\
\hline BOBVIY01 & $\mathrm{V}$ & 300 & 0.0001 & 1 & 232.927 & 232.772 & 232.772 & 0 & 0 & 232.772 \\
\hline BOBVIY09 & VI & 296 & 0.0001 & 2 & 434.963 & 434.839 & 217.420 & 0 & 0 & 217.420 \\
\hline BOBVIY04 & VII & 296 & 0.20 & 4 & 867.428 & 867.181 & 216.795 & 0 & 0 & 216.795 \\
\hline BOBVIY05 & VIII & 296 & 0.20 & 4 & 857.709 & 857.465 & 214.366 & 0 & 0 & 214.366 \\
\hline BOBVIY06 & IX & 296 & 0.50 & 4 & 837.640 & 837.401 & 209.350 & 0 & 0 & 209.350 \\
\hline BOBVIY07 & IX & 296 & 1.00 & 4 & 805.759 & 805.529 & 201.382 & 0 & 0 & 201.382 \\
\hline EVUQAO & $1 \mathrm{H} \alpha$ & 296 & 0.50 & 40 & 9794.255 & 9791.464 & 244.787 & 1 & 21.03 & 223.76 \\
\hline EVUQAO01 & $1 \mathrm{H} \beta$ & 296 & 1.40 & 4 & 856.679 & 856.435 & 214.109 & 1 & 19.96 & 194.15 \\
\hline \multicolumn{11}{|c|}{ Thiourea } \\
\hline THIOUR13 & $\mathrm{V}$ & 295 & 0.0001 & 4 & 360.155 & 360.087 & 90.022 & 0 & 0 & 90.022 \\
\hline THIOUR17 & VI & 295 & 0.28 & 4 & 347.342 & 347.276 & 86.819 & 0 & 0 & 86.819 \\
\hline THIOUR18 & VI & 295 & 0.38 & 12 & 1020.543 & 1020.349 & 85.029 & 0 & 0 & 85.029 \\
\hline THIOUR19 & VI & 295 & 0.97 & 12 & 973.683 & 973.498 & 81.125 & 0 & 0 & 81.125 \\
\hline NEXSUG01 & $0.67 \mathrm{H}$ & 296 & 0.70 & 12 & 1154.473 & 1154.144 & 96.179 & 0.67 & 13.93 & 82.25 \\
\hline NEXSUG & $0.67 \mathrm{H}$ & 296 & 0.95 & 12 & 1132.561 & 1132.238 & 94.353 & 0.67 & 13.73 & 80.63 \\
\hline NEXSIU01 & $1 \mathrm{H}$ & 296 & 0.60 & 4 & 425.817 & 425.696 & 106.424 & 1 & 20.91 & 85.52 \\
\hline NEXSIU & $1 \mathrm{H}$ & 296 & 1.20 & 4 & 411.966 & 411.849 & 102.962 & 1 & 20.19 & 82.77 \\
\hline
\end{tabular}




\begin{tabular}{|c|c|c|c|c|c|c|c|c|c|c|}
\hline \multicolumn{11}{|c|}{ IN SITU-FORMED HYDRATES (continue) } \\
\hline 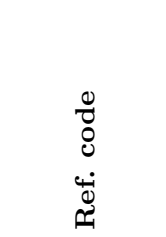 & 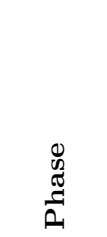 & $\underset{E}{E}$ & $\underbrace{\overparen{\sigma}}_{0}$ & $\mathbf{N}$ & 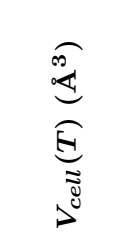 & 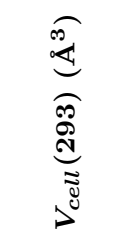 & $\underset{D^{N}}{\stackrel{0}{0^{2}}}$ & Z & 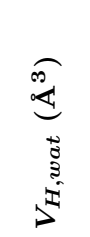 & 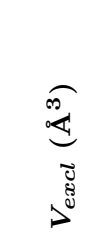 \\
\hline \multicolumn{11}{|c|}{ dabco2HBr } \\
\hline PASWUD & $\beta$ & 296 & 0.0001 & 4 & 999.174 & 998.889 & 249.722 & 0 & 0 & 249.722 \\
\hline PASXAK & $0.5 \mathrm{H} \alpha$ & 296 & 0.0001 & 8 & 2030.353 & 2029.774 & 253.722 & 0.5 & 10.82 & 242.901 \\
\hline PASXAK01 & $0.5 \mathrm{H} \beta$ & 296 & 0.0001 & 4 & 1028.137 & 1027.844 & 256.961 & 0.5 & 10.82 & 246.140 \\
\hline PASXIS & $1 \mathrm{H} \alpha$ & 296 & 0.0001 & 8 & 2080.335 & 2079.742 & 259.968 & 1 & 21.64 & 238.326 \\
\hline PASXIS01 & $1 \mathrm{H} \beta$ & 296 & 0.48 & 4 & 989.198 & 988.916 & 247.229 & 1 & 21.05 & 226.178 \\
\hline PASXIS02 & $1 \mathrm{H} \gamma$ & 296 & 1.50 & 2 & 450.385 & 450.257 & 225.128 & 1 & 19.85 & 205.282 \\
\hline PASXIS03 & $1 \mathrm{H} \delta$ & 296 & 0.78 & 2 & 490.151 & 490.011 & 245.006 & 1 & 20.69 & 224.316 \\
\hline
\end{tabular}


Appendix $C$

\begin{tabular}{|c|c|c|c|c|c|c|c|c|c|c|}
\hline \multicolumn{11}{|c|}{ COMPRESSED HYDRATES } \\
\hline 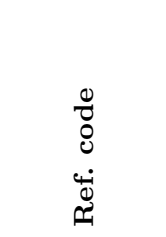 & 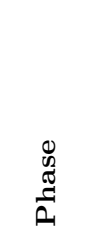 & $\underset{E-1}{\mathcal{E}}$ & 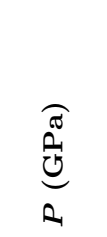 & $\mathbf{N}$ & 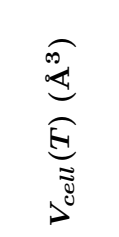 & 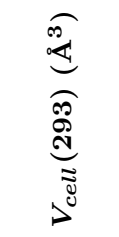 & $\overbrace{\underbrace{N}}^{\stackrel{\infty}{0^{N}}}$ & Z & 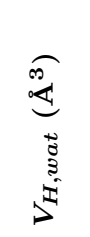 & $\begin{array}{c}\widetilde{m} \\
\widetilde{0} \\
\widetilde{\sigma} \\
\nu^{\circ}\end{array}$ \\
\hline \multicolumn{11}{|c|}{ L-Cysteic acid } \\
\hline CYSTEA & I & 295 & 0.0001 & 4 & 631.180 & 631.060 & 157.765 & 0 & 0 & 157.765 \\
\hline CYSTAC02 & $1 \mathrm{H}$ & 293 & 0.0001 & 4 & 698.386 & 698.386 & 174.597 & 1 & 21.64 & 152.95 \\
\hline CYSTAC03 & $1 \mathrm{H}$ & 293 & 0.20 & 4 & 693.060 & 693.060 & 173.265 & 1 & 21.39 & 151.87 \\
\hline CYSTAC04 & $1 \mathrm{H}$ & 293 & 1.20 & 4 & 665.239 & 665.239 & 166.310 & 1 & 20.19 & 146.12 \\
\hline CYSTAC05 & $1 \mathrm{H}$ & 293 & 2.80 & 4 & 633.205 & 633.205 & 158.301 & 1 & 18.41 & 139.89 \\
\hline CYSTAC06 & $1 \mathrm{H}$ & 293 & 4.50 & 4 & 611.870 & 611.870 & 152.968 & 1 & 16.69 & 136.28 \\
\hline CYSTAC07 & $1 \mathrm{H}$ & 293 & 5.80 & 4 & 595.468 & 595.468 & 148.867 & 1 & 15.48 & 133.38 \\
\hline CYSTAC08 & $1 \mathrm{H}$ & 293 & 6.80 & 4 & 589.954 & 589.954 & 147.489 & 1 & 14.61 & 132.87 \\
\hline \multicolumn{11}{|c|}{ (S)-4-Sulfo-L-phenylalanine } \\
\hline VENLUW & I & 293 & 0.0001 & 2 & 526.627 & 526.627 & 263.314 & 0 & 0 & 263.314 \\
\hline IFIZIG & $1 \mathrm{H} \mathrm{I}$ & 295 & 0.0001 & 2 & 576.668 & 576.558 & 288.279 & 1 & 21.64 & 266.64 \\
\hline IFIZIG02 & $1 \mathrm{H} \mathrm{I}$ & 293 & 0.20 & 2 & 571.119 & 571.119 & 285.560 & 1 & 21.39 & 264.17 \\
\hline IFIZIG03 & $1 \mathrm{H} \mathrm{I}$ & 293 & 1.00 & 2 & 545.135 & 545.135 & 272.568 & 1 & 20.43 & 252.14 \\
\hline IFIZIG04 & $1 \mathrm{H} \mathrm{II}$ & 293 & 2.50 & 4 & 974.611 & 974.611 & 243.653 & 1 & 18.73 & 224.92 \\
\hline IFIZIG05 & $1 \mathrm{H} \mathrm{II}$ & 293 & 4.00 & 4 & 936.253 & 936.253 & 234.063 & 1 & 17.18 & 216.88 \\
\hline \multicolumn{11}{|c|}{ DL-Alaninium oxalate } \\
\hline NELPUP & $\mathrm{I}$ & 293 & 0.0001 & 4 & 793.931 & 793.931 & 198.483 & 0 & 0 & 198.483 \\
\hline IMEGIR & $1 \mathrm{H}$ & 300 & 0.0001 & 4 & 860.043 & 859.471 & 214.868 & 1 & 21.64 & 193.23 \\
\hline IMEGIR01 & $1 \mathrm{H}$ & 293 & 0.30 & 4 & 839.975 & 839.975 & 209.994 & 1 & 21.27 & 188.72 \\
\hline IMEGIR02 & $1 \mathrm{H}$ & 293 & 0.50 & 4 & 830.109 & 830.109 & 207.527 & 1 & 21.03 & 186.50 \\
\hline IMEGIR03 & $1 \mathrm{H}$ & 293 & 1.10 & 4 & 805.899 & 805.899 & 201.475 & 1 & 20.31 & 181.16 \\
\hline IMEGIR04 & $1 \mathrm{H}$ & 293 & 1.50 & 4 & 791.348 & 791.348 & 197.837 & 1 & 19.85 & 177.99 \\
\hline IMEGIR05 & $1 \mathrm{H}$ & 293 & 2.40 & 4 & 745.952 & 745.952 & 186.488 & 1 & 18.84 & 167.65 \\
\hline IMEGIR06 & $1 \mathrm{H}$ & 293 & 3.10 & 4 & 725.179 & 725.179 & 181.295 & 1 & 18.10 & 163.20 \\
\hline IMEGIR07 & $1 \mathrm{H}$ & 293 & 3.60 & 4 & 713.457 & 713.457 & 178.364 & 1 & 17.58 & 160.78 \\
\hline IMEGIR08 & $1 \mathrm{H}$ & 293 & 4.30 & 4 & 700.812 & 700.812 & 175.203 & 1 & 16.88 & 158.32 \\
\hline
\end{tabular}




\begin{tabular}{|c|c|c|c|c|c|c|c|c|c|c|}
\hline \multicolumn{11}{|c|}{ COMPRESSED HYDRATES (continue) } \\
\hline 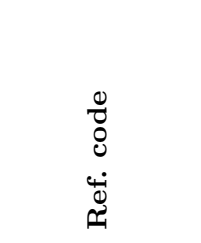 & $\begin{array}{c}0 \\
0 \\
\frac{0}{\sigma} \\
\frac{\pi}{n}\end{array}$ & $\underbrace{E}_{E-1}$ & 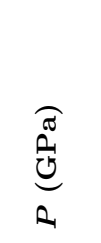 & $\mathbf{N}$ & 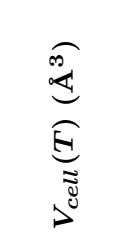 & 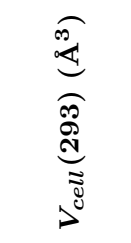 & $\begin{array}{l}\stackrel{\infty}{0} \\
\dot{0}^{N}\end{array}$ & Z & 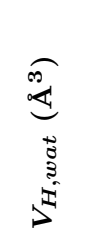 & 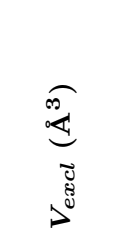 \\
\hline \multicolumn{11}{|c|}{ Betaine } \\
\hline WEMWEQ & I & 295 & 0.0001 & 4 & 611.612 & 611.496 & 152.874 & 0 & 0 & 152.874 \\
\hline WEMWEQ02 & I & 293 & 0.80 & 4 & 577.640 & 577.640 & 144.410 & 0 & 0 & 144.410 \\
\hline WEMWEQ03 & I & 293 & 1.40 & 4 & 558.889 & 558.889 & 139.722 & 0 & 0 & 139.722 \\
\hline WEMWEQ04 & I & 293 & 2.20 & 4 & 540.905 & 540.905 & 135.226 & 0 & 0 & 135.226 \\
\hline WEMWEQ05 & I & 293 & 3.20 & 4 & 522.441 & 522.441 & 130.610 & 0 & 0 & 130.610 \\
\hline WEMWEQ06 & I & 293 & 4.10 & 4 & 509.535 & 509.535 & 127.384 & 0 & 0 & 127.384 \\
\hline KICCOO & $1 \mathrm{H}$ & 295 & 0.0001 & 8 & 1428.353 & 1428.082 & 178.510 & 1 & 21.64 & 156.87 \\
\hline KICCOO03 & $1 \mathrm{H}$ & 293 & 0.10 & 8 & 1404.275 & 1404.275 & 175.534 & 1 & 21.52 & 154.02 \\
\hline KICCOO04 & $1 \mathrm{H}$ & 293 & 1.60 & 8 & 1284.940 & 1284.940 & 160.618 & 1 & 19.73 & 140.88 \\
\hline KICCOO05 & $1 \mathrm{H}$ & 293 & 2.90 & 8 & 1227.902 & 1227.902 & 153.488 & 1 & 18.31 & 135.18 \\
\hline KICCOO06 & $1 \mathrm{H}$ & 293 & 4.00 & 8 & 1190.086 & 1190.086 & 148.761 & 1 & 17.18 & 131.58 \\
\hline KICCOO07 & $1 \mathrm{H}$ & 293 & 4.90 & 8 & 1163.267 & 1163.267 & 145.408 & 1 & 16.31 & 129.10 \\
\hline \multicolumn{11}{|c|}{ L-Serine } \\
\hline LSERIN10 & I & 295 & 0.0001 & 4 & 448.776 & 448.691 & 112.173 & 0 & 0 & 112.173 \\
\hline LSERIN41 & I & 293 & 0.10 & 4 & 442.267 & 442.267 & 110.567 & 0 & 0 & 110.567 \\
\hline LSERIN42 & I & 293 & 0.30 & 4 & 443.614 & 443.614 & 110.904 & 0 & 0 & 110.904 \\
\hline LSERIN43 & I & 293 & 0.50 & 4 & 436.602 & 436.602 & 109.151 & 0 & 0 & 109.151 \\
\hline LSERIN44 & I & 293 & 0.80 & 4 & 427.346 & 427.346 & 106.837 & 0 & 0 & 106.837 \\
\hline LSERIN45 & I & 293 & 1.00 & 4 & 427.399 & 427.399 & 106.850 & 0 & 0 & 106.850 \\
\hline LSERIN12 & I & 293 & 1.40 & 4 & 419.181 & 419.181 & 104.795 & 0 & 0 & 104.795 \\
\hline LSERIN46 & I & 293 & 2.10 & 4 & 410.549 & 410.549 & 102.637 & 0 & 0 & 102.637 \\
\hline LSERIN13 & I & 293 & 2.90 & 4 & 404.488 & 404.488 & 101.122 & 0 & 0 & 101.122 \\
\hline LSERIN47 & I & 293 & 3.10 & 4 & 407.435 & 407.435 & 101.859 & 0 & 0 & 101.859 \\
\hline LSERIN48 & I & 293 & 4.00 & 4 & 395.111 & 395.111 & 98.778 & 0 & 0 & 98.778 \\
\hline LSERIN14 & I & 293 & 4.10 & 4 & 395.750 & 395.750 & 98.938 & 0 & 0 & 98.938 \\
\hline LSERIN26 & I & 293 & 4.20 & 4 & 392.807 & 392.807 & 98.202 & 0 & 0 & 98.202 \\
\hline LSERIN15 & I & 293 & 4.80 & 4 & 390.090 & 390.090 & 97.523 & 0 & 0 & 97.523 \\
\hline LSERIN03 & II & 295 & 0.0001 & 8 & 899.918 & 899.747 & 112.468 & 0 & 0 & 112.468 \\
\hline LSERIN49 & II & 293 & 5.00 & 4 & 374.873 & 374.873 & 93.718 & 0 & 0 & 93.718 \\
\hline LSERMH10 & $1 \mathrm{H}$ & 295 & 0.0001 & 4 & 554.179 & 554.074 & 138.518 & 1 & 21.64 & 116.88 \\
\hline LSERMH11 & $1 \mathrm{H}$ & 293 & 1.00 & 4 & 522.922 & 522.922 & 130.731 & 1 & 20.43 & 110.30 \\
\hline LSERMH12 & $1 \mathrm{H}$ & 293 & 2.60 & 4 & 494.272 & 494.272 & 123.568 & 1 & 18.63 & 104.94 \\
\hline LSERMH13 & $1 \mathrm{H}$ & 293 & 3.70 & 4 & 476.027 & 476.027 & 119.007 & 1 & 17.48 & 101.53 \\
\hline LSERMH15 & $1 \mathrm{H}$ & 293 & 4.50 & 4 & 463.213 & 463.213 & 115.803 & 1 & 16.69 & 99.11 \\
\hline \multicolumn{11}{|c|}{ Oxalic acid } \\
\hline OXALAC06 & $\alpha$ & 295 & 0.0001 & 4 & 313.884 & 313.824 & 78.456 & 0 & 0 & 78.456 \\
\hline OXALAC07 & $\beta$ & 298 & 0.0001 & 2 & 157.020 & 156.945 & 78.473 & 0 & 0 & 78.473 \\
\hline OXACDH11 & $2 \mathrm{H}$ & 295 & 0.0001 & 2 & 255.390 & 255.341 & 127.671 & 2 & 43.28 & 84.39 \\
\hline OXACDH36 & $2 \mathrm{H}$ & 295 & 1.60 & 2 & 226.518 & 226.475 & 113.237 & 2 & 39.47 & 73.77 \\
\hline OXACDH37 & $2 \mathrm{H}$ & 295 & 3.60 & 2 & 206.399 & 206.360 & 103.180 & 2 & 35.16 & 68.02 \\
\hline
\end{tabular}




\begin{tabular}{|c|c|c|c|c|c|c|c|c|c|c|}
\hline \multicolumn{11}{|c|}{ AMBIENT-PRESSURE HYDRATES } \\
\hline $\begin{array}{l}0 \\
0 \\
0 \\
\dot{0} \\
\dot{0} \\
\dot{0}\end{array}$ & 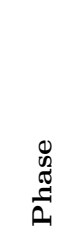 & $\underbrace{\mathcal{E}}_{E}$ & 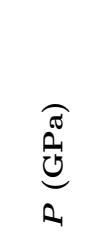 & $\mathbf{N}$ & 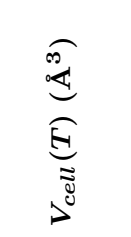 & 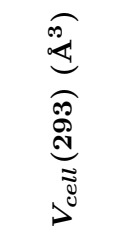 & $\overbrace{D^{N}}^{\stackrel{\infty}{0^{2}}}$ & Z & 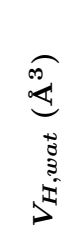 & 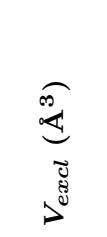 \\
\hline \multicolumn{11}{|c|}{ Ethylenediamine } \\
\hline ETDIAM14 & I $\alpha$ & 296 & 0.15 & 2 & 182.918 & 182.866 & 91.433 & 0 & 0 & 91.433 \\
\hline ETDIAM15 & I $\beta$ & 296 & 0.30 & 2 & 168.946 & 168.898 & 84.449 & 0 & 0 & 84.449 \\
\hline ETDIAM16 & I $\beta$ & 296 & 1.10 & 2 & 161.644 & 161.598 & 80.799 & 0 & 0 & 80.799 \\
\hline ETDIAM17 & $\mathrm{I} \gamma$ & 296 & 1.50 & 4 & 321.419 & 321.327 & 80.332 & 0 & 0 & 80.332 \\
\hline ETDIAM18 & III & 296 & 0.20 & 2 & 179.477 & 179.426 & 89.713 & 0 & 0 & 89.713 \\
\hline ETDIAM19 & III & 296 & 0.30 & 2 & 173.214 & 173.165 & 86.582 & 0 & 0 & 86.582 \\
\hline ETDIAM20 & III & 296 & 0.50 & 2 & 172.526 & 172.477 & 86.238 & 0 & 0 & 86.238 \\
\hline SOWSUS & $1 \mathrm{H}$ & 268 & 0.0001 & 4 & 480.569 & 481.710 & 120.428 & 1 & 21.64 & 98.79 \\
\hline GIXDAS & $2 \mathrm{H}$ & 123 & 0.0001 & 4 & 555.789 & 564.765 & 141.191 & 2 & 43.28 & 97.91 \\
\hline \multicolumn{11}{|c|}{ Glycylglycine } \\
\hline GLYGLY14 & $\alpha$ & 293 & 0.0001 & 4 & 578.692 & 578.692 & 144.673 & 0 & 0 & 144.673 \\
\hline GLYGLY15 & $\alpha$ & 293 & 1.40 & 4 & 532.441 & 532.441 & 133.110 & 0 & 0 & 133.110 \\
\hline GLYGLY16 & $\alpha$ & 293 & 3.00 & 4 & 511.844 & 511.844 & 127.961 & 0 & 0 & 127.961 \\
\hline GLYGLY17 & $\alpha$ & 293 & 3.70 & 4 & 499.272 & 499.272 & 124.818 & 0 & 0 & 124.818 \\
\hline GLYGLY18 & $\alpha$ & 293 & 4.70 & 4 & 492.861 & 492.861 & 123.215 & 0 & 0 & 123.215 \\
\hline GLYGLY01 & $\beta$ & 295 & 0.0001 & 8 & 1159.746 & 1159.526 & 144.941 & 0 & 0 & 144.941 \\
\hline GLYGLY08 & $\gamma$ & 295 & 0.0001 & 4 & 583.783 & 583.672 & 145.918 & 0 & 0 & 145.918 \\
\hline РОTРЕT03 & $1.5 \mathrm{H}$ & 295 & 0.0001 & 8 & 1391.156 & 1390.892 & 173.861 & 1.5 & 32.46 & 141.40 \\
\hline \multicolumn{11}{|c|}{ Phenol } \\
\hline PHENOL02 & I & 295 & 0.0001 & 6 & 826.108 & 825.951 & 137.659 & 0 & 0 & 137.659 \\
\hline PHENOL11 & II & 293 & 0.16 & 6 & 756.419 & 756.419 & 126.070 & 0 & 0 & 126.070 \\
\hline PHOLHH & $0.5 \mathrm{H}$ & 295 & 0.0001 & 8 & 1132.346 & 1132.131 & 141.516 & 0.5 & 10.82 & 130.70 \\
\hline \multicolumn{11}{|c|}{ Cyclohexane-1,2-diamine } \\
\hline PIWXEY & I & 220 & 0.0001 & 2 & 341.921 & 344.292 & 172.146 & 0 & 0 & 172.146 \\
\hline PIWXEY02 & I & 296 & 0.36 & 2 & 346.825 & 346.726 & 173.363 & 0 & 0 & 173.363 \\
\hline PIWXEY03 & I & 296 & 0.52 & 2 & 329.784 & 329.690 & 164.845 & 0 & 0 & 164.845 \\
\hline PIWXEY04 & I & 296 & 0.65 & 2 & 324.818 & 324.725 & 162.363 & 0 & 0 & 162.363 \\
\hline PIWXEY05 & I & 296 & 1.19 & 2 & 311.288 & 311.199 & 155.600 & 0 & 0 & 155.600 \\
\hline PIWXEY06 & I & 296 & 2.04 & 2 & 291.039 & 290.956 & 145.478 & 0 & 0 & 145.478 \\
\hline LAJGUA & $1 \mathrm{H}$ & 100 & 0.0001 & 8 & 1552.126 & 1580.584 & 197.573 & 1 & 21.64 & 175.93 \\
\hline \multicolumn{11}{|c|}{ Pyridine } \\
\hline PYRDNA01 & I & 153 & 0.0001 & 16 & 1784.225 & 1807.955 & 112.997 & 0 & 0 & 112.997 \\
\hline PYRDNA06 & I & 295 & 1.23 & 16 & 1665.516 & 1665.200 & 104.075 & 0 & 0 & 104.075 \\
\hline PYRDNA03 & II & 293 & 1.10 & 4 & 413.962 & 413.962 & 103.491 & 0 & 0 & 103.491 \\
\hline PYRTHA10 & $3 \mathrm{H}$ & 223 & 0.0001 & 8 & 1507.175 & 1517.198 & 189.650 & 3 & 67.10 & 122.55 \\
\hline
\end{tabular}




\begin{tabular}{|c|c|c|c|c|c|c|c|c|c|c|}
\hline \multicolumn{11}{|c|}{ AMBIENT-PRESSURE HYDRATES (continue) } \\
\hline 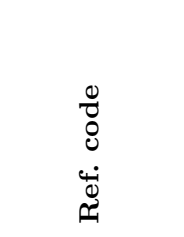 & 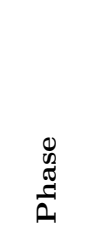 & $\underbrace{\widehat{E}}_{-1}$ & 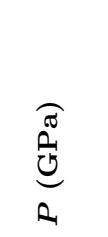 & $\mathbf{N}$ & 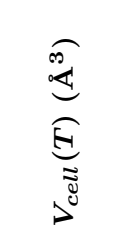 & 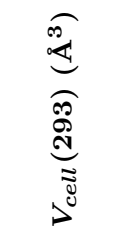 & $\overbrace{D^{N}}^{\stackrel{\infty}{N}}$ & Z & 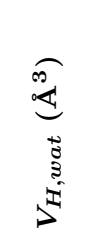 & 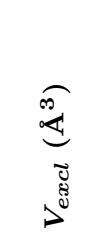 \\
\hline \multicolumn{11}{|c|}{$\alpha$-D-Glucose } \\
\hline GLUCSA10 & I & 295 & 0.0001 & 4 & 765.919 & 765.773 & 191.443 & 0 & 0 & 191.443 \\
\hline GLUCSA13 & I & 295 & 0.27 & 4 & 718.518 & 718.381 & 179.595 & 0 & 0 & 179.595 \\
\hline GLUCSA11 & I & 295 & 0.88 & 4 & 733.265 & 733.126 & 183.281 & 0 & 0 & 183.281 \\
\hline GLUCSA12 & I & 295 & 1.12 & 4 & 722.884 & 722.747 & 180.687 & 0 & 0 & 180.687 \\
\hline GLUCSA14 & I & 295 & 1.80 & 4 & 707.518 & 707.384 & 176.846 & 0 & 0 & 176.846 \\
\hline GLUCSA15 & I & 295 & 2.60 & 4 & 685.682 & 685.552 & 171.388 & 0 & 0 & 171.388 \\
\hline GLUCSA16 & I & 295 & 3.21 & 4 & 675.414 & 675.286 & 168.821 & 0 & 0 & 168.821 \\
\hline GLUCSA23 & I & 295 & 4.00 & 4 & 657.831 & 657.706 & 164.427 & 0 & 0 & 164.427 \\
\hline GLUCSA24 & I & 295 & 4.20 & 4 & 651.831 & 651.707 & 162.927 & 0 & 0 & 162.927 \\
\hline GLUCMH11 & $1 \mathrm{H}$ & 295 & 0.0001 & 2 & 430.674 & 430.592 & 215.296 & 1 & 21.64 & 193.65 \\
\hline \multicolumn{11}{|c|}{ MDMA } \\
\hline NEDMIS01 & I & 293 & 0.0001 & 4 & 1217.768 & 1217.768 & 304.442 & 0 & 0 & 304.442 \\
\hline NEDMIS02 & I & 293 & 1.14 & 4 & 1129.311 & 1129.311 & 282.328 & 0 & 0 & 282.328 \\
\hline NEDMIS03 & I & 293 & 2.20 & 4 & 1053.087 & 1053.087 & 263.272 & 0 & 0 & 263.272 \\
\hline NEDMIS04 & I & 293 & 3.84 & 4 & 970.304 & 970.304 & 242.576 & 0 & 0 & 242.576 \\
\hline NEDMIS05 & I & 293 & 4.42 & 4 & 952.540 & 952.540 & 238.135 & 0 & 0 & 238.135 \\
\hline TOKYIC & $1 \mathrm{H}$ & 120 & 0.0001 & 4 & 1313.181 & 1334.763 & 333.691 & 1 & 21.64 & 312.05 \\
\hline \multicolumn{11}{|c|}{ tert-Butanol } \\
\hline VATSAK02 & II & 220 & 0.0001 & 18 & 2382.977 & 2399.503 & 133.306 & 0 & 0 & 133.306 \\
\hline VATSAK01 & II & 293 & 0.85 & 18 & 2155.243 & 2155.243 & 119.736 & 0 & 0 & 119.736 \\
\hline VATSAK & IV & 295 & 0.0001 & 6 & 874.408 & 874.242 & 145.707 & 0 & 0 & 145.707 \\
\hline LEBJUX & $2 \mathrm{H}$ & 123 & 0.0001 & 2 & 362.308 & 368.159 & 184.080 & 2 & 43.28 & 140.80 \\
\hline LEBKEI & $7 \mathrm{H}$ & 123 & 0.0001 & 4 & 1275.806 & 1296.410 & 324.103 & 7 & 151.50 & 172.61 \\
\hline \multicolumn{11}{|c|}{ 1H-Tetrazole-5-amine } \\
\hline EJIQEU & $\mathrm{I}$ & 298 & 0.0001 & 4 & 337.289 & 337.129 & 84.282 & 0 & 0 & 84.282 \\
\hline EJIQEU01 & I & 298 & 4.80 & 4 & 283.494 & 283.359 & 70.840 & 0 & 0 & 70.840 \\
\hline AMTETZ01 & $1 \mathrm{H}$ & 295 & 0.0001 & 4 & 455.978 & 455.891 & 113.973 & 1 & 21.64 & 92.33 \\
\hline \multicolumn{11}{|c|}{ Hexanitrohexa-Azaisowurtzitane } \\
\hline PUBMUU01 & $\beta$ & 295 & 0.0001 & 4 & 1465.981 & 1465.702 & 366.426 & 0 & 0 & 366.426 \\
\hline PUBMUU06 & $\gamma$ & 295 & 0.0001 & 4 & 1517.408 & 1517.120 & 379.280 & 0 & 0 & 379.280 \\
\hline PUBMUU05 & $\epsilon$ & 295 & 0.0001 & 4 & 1432.021 & 1431.749 & 357.937 & 0 & 0 & 357.937 \\
\hline PUBMUU23 & $\zeta$ & 293 & 3.30 & 4 & 1279.097 & 1279.097 & 319.774 & 0 & 0 & 319.774 \\
\hline PUBMII01 & $0.25 \mathrm{H}$ & 295 & 0.0001 & 8 & 2985.274 & 2984.707 & 373.088 & 0.25 & 5.59 & 367.50 \\
\hline FEFRIS & $0.75 \mathrm{H}$ & 295 & 0.0001 & 8 & 3021.867 & 3021.293 & 377.662 & 0.57 & 12.75 & 364.91 \\
\hline
\end{tabular}




\section{List of available determinations of $\beta$-cyclodextrin form I}

\begin{tabular}{|c|c|c|c|c|}
\hline Ref. code & $\mathbf{T}(\mathbf{K})^{a}$ & $R(\%)$ & $N$ & Reference \\
\hline BCDEXD & $\mathrm{RT}$ & 7.1 & 12 & K. Lindner and W. Saenger, Angew. Chem. Int. Ed., 1978, 17, 694. \\
\hline BCDEXD01 & 120 & 13 & 12 & J. J. Stezowski and J. M. Maclennan, ACA Ser. 2, 1980, 7, 24a. \\
\hline BCDEXD02 & $\mathrm{RT}$ & 7.2 & 12 & T. Fujiwara et al., J. Chem. Soc. Jpn., 1983, 181. \\
\hline BCDEXD03 & $\mathrm{RT}$ & 6.1 & 12.26 & T. Steiner and G. Koellner, J. Am. Chem. Soc., 1994, 116, 5122. \\
\hline BCDEXD04 & $\mathrm{RT}$ & 5.9 & 11.56 & T. Steiner and G. Koellner, J. Am. Chem. Soc., 1994, 116, 5122. \\
\hline BCDEXD05 & $\mathrm{RT}$ & 5.9 & 11.89 & T. Steiner and G. Koellner, J. Am. Chem. Soc., 1994, 116, 5122. \\
\hline BCDEXD10 & $\mathrm{RT}$ & 7.3 & 12 & K. Lindner and W. Saenger, Carbohydr. Res., 1982, 99, 103. \\
\hline BCDEXD11 & $\mathrm{RT}$ & - & 12 & J. A. Hamilton et al., Acta. Cryst., 1968, B24, 1560. \\
\hline BUVSEQ & $\mathrm{RT}$ & 8.0 & 11 & T. Fujiwara et al., J. Chem. Soc. Jpn., 1983, 181. \\
\hline BUVSEQ01 & $\mathrm{RT}$ & 6.0 & 11.19 & T. Steiner and G. Koellner, J. Am. Chem. Soc., 1994, 116, 5122. \\
\hline BUVSEQ04 & 100 & - & 11 & C. Kurokawa et al., Supramo. Chem., 2004, 16, 381. \\
\hline POBRON & $\mathrm{RT}$ & 6.8 & 9.35 & T. Steiner and G. Koellner, J. Am. Chem. Soc., 1994, 116, 5122. \\
\hline POBRON01 & $\mathrm{RT}$ & 7.49 & 9 & L. Damodharan et al., Mol. Cyst. Liq. Cryst., 2004, 423, 17. \\
\hline POBRON02 & $\mathrm{RT}$ & - & - & J. Szejtli and Z. Budai, Acta Chim. Acad. Sci. Hung., 1977, 94, 383. \\
\hline POBRUT & $\mathrm{RT}$ & - & 1 & T. Steiner and G. Koellner, J. Am. Chem. Soc., 1994, 116, 5122. \\
\hline POBRUT01 & $\mathrm{RT}$ & - & 1 & T. Steiner and G. Koellner, J. Am. Chem. Soc., 1994, 116, 5122. \\
\hline POBRUT02 & $\mathrm{RT}$ & - & 1 & T. Steiner and G. Koellner, J. Am. Chem. Soc., 1994, 116, 5122. \\
\hline POBRUT03 & $\mathrm{RT}$ & - & 1 & T. Steiner and G. Koellner, J. Am. Chem. Soc., 1994, 116, 5122. \\
\hline SUJDAD & 110 & 3.2 & 10.41 & R. W. Seidel and B. B. Koleva, Acta Cryst., 2009, E65, o3162. \\
\hline TUSHUL & $\mathrm{RT}$ & 7.6 & 7.5 & J.-Y. Li et al., Carbohydr. Res., 2010, 345, 685. \\
\hline
\end{tabular}

${ }^{a}$ RT stands for room temperature, between 283 and $303 \mathrm{~K}$ according to the CSD. 


\section{E List of all the structures included in the XPAC calculation}

$P 2_{1}$ structures used in the XPAC calculation with unit-cell parameters, guest, and hydration number $(N)$. Structures with the reference code in bold are those in which the $a$ axis is longer than the $c$ axis. In all the cases the $b$ axis is the shortest axis, with the exception of the reference code in italics.

\begin{tabular}{|c|c|c|c|c|c|c|c|}
\hline Ref. code & $a(\AA)$ & $b(\AA)$ & $c(\AA)$ & $\boldsymbol{\beta}\left({ }^{\circ}\right)$ & $V\left(\AA^{3}\right)$ & guest & $N$ \\
\hline ARUXIU & $15.1711(3)$ & $10.16870(10)$ & $20.9862(4)$ & 110.92 & 3024.13 & formic acid & 7.7 \\
\hline ARUXOA & $15.263(4)$ & $10.157(2)$ & $21.044(5)$ & $110.698(4)$ & 3051.81 & acetic acid & 7.7 \\
\hline BCDEXD03 & $21.283(5)$ & $10.322(1)$ & $15.092(5)$ & $112.41(1)$ & 3065.07 & & 12.26 \\
\hline BCDEXD04 & $21.161(7)$ & $10.254(1)$ & $15.110(7)$ & $111.91(2)$ & 3041.83 & & 11.56 \\
\hline BCDEXD05 & $21.233(5)$ & $10.294(1)$ & $15.103(4)$ & $112.22(1)$ & 3055.96 & & 11.89 \\
\hline BCDEXD10 & $21.29(2)$ & $10.33(1)$ & $15.10(2)$ & $112.3(5)$ & 3072.51 & & 12 \\
\hline BOBPEN & $21.25(3)$ & $10.28(2)$ & $15.30(2)$ & $113.25(9)$ & 3070.86 & hydrogen iodide & 8 \\
\hline BOBPIR & $21.03(3)$ & $10.11(2)$ & $15.33(2)$ & $111.02(9)$ & 3042.47 & methanol & 6.5 \\
\hline BUVSEQ01 & $21.080(7)$ & $10.197(1)$ & $15.131(6)$ & $111.58(2)$ & 3024.47 & & 11.19 \\
\hline DEBGOG & $15.356(3)$ & $10.101(2)$ & $21.287(3)$ & $112.81(1)$ & 3043.63 & benzyl alcohol & 5 \\
\hline DEWCOY & $15.4244(16)$ & $10.1574(11)$ & $20.557(2)$ & $110.074(2)$ & 3025.04 & benzamide & 6 \\
\hline DIRVOP & $15.285(3)$ & $10.345(2)$ & $20.118(2)$ & $102.14(1)$ & 3109.98 & hexamethylenetetramine & 6 \\
\hline GUXzoO & $15.1220(4)$ & $10.3350(2)$ & $20.9150(3)$ & $109.575(3)$ & 3079.8 & m-aminophenol & 7.5 \\
\hline HAQNUK & $15.274(12)$ & $10.178(8)$ & $20.961(16)$ & $110.251(7)$ & 3057.14 & 1,4-dioxane & 7 \\
\hline IZALEB & $15.0678(9)$ & $10.2126(6)$ & $20.9653(13)$ & $111.7473(12)$ & 2996.55 & nicotinic acid & 7.65 \\
\hline KIJSEC & $15.1977(7)$ & $10.1763(5)$ & $20.6943(6)$ & $109.239(4)$ & 3021.76 & succinic acid & 7 \\
\hline KOBRIC & $15.223(5)$ & $10.578(3)$ & $20.204(6)$ & $108.37(7)$ & 3087.64 & potassium hydroxide & 8 \\
\hline KUTKOZ & $21.199(12)$ & $9.973(3)$ & $15.271(8)$ & $110.87(3)$ & 3016.74 & 1,4-butanediol & 6.25 \\
\hline LADBAV & $15.15(2)$ & $10.343(15)$ & $20.93(3)$ & $109.69(4)$ & 3087.89 & pentanedioic acid & 4.2 \\
\hline LADQOX & $20.82(1)$ & $10.366(6)$ & $14.968(9)$ & $110.12(4)$ & 3033.26 & diaqua-dichloro-copper(II) & 8.9 \\
\hline MIFHAK & $15.231(3)$ & $10.068(3)$ & $21.117(3)$ & $110.16(5)$ & 3039.81 & squaric acid & 6.65 \\
\hline NUFVEQ & $20.8314(9)$ & $10.2937(6)$ & $15.2155(8)$ & $110.733(2)$ & 3051.4 & pyrazinamide & 5.5 \\
\hline NUFVIU & $20.95040(10)$ & $10.2493(2)$ & $15.1424(3)$ & $108.7630(10)$ & 3078.69 & piperazine & 7.2 \\
\hline PIJGIY & $21.212(1)$ & $10.021(3)$ & $15.208(1)$ & $111.47(3)$ & 3008.38 & ethylene glycol & 8 \\
\hline PIJGOE & $21.322(6)$ & $9.954(1)$ & $15.251(6)$ & $111.20(1)$ & 3017.8 & glycerol & 7.2 \\
\hline POBRON & $20.857(6)$ & $10.158(1)$ & $15.140(6)$ & $110.94(1)$ & 2995.79 & & 9.35 \\
\hline POBRON01 & $15.107(7)$ & $10.242(3)$ & $20.911(8)$ & $110.27(3)$ & 3035.1 & & 9 \\
\hline POVSIC & $20.042(6)$ & $10.378(2)$ & $15.123(9)$ & $102.30(2)$ & 3073.32 & trans-cyclohexane-1,4-diol & 5.4 \\
\hline SUJDAD & $20.8353(4)$ & $9.93970(10)$ & $15.2043(3)$ & $110.630(2)$ & 2946.85 & & 10.41 \\
\hline TUSHUL & $15.1667(5)$ & $10.1850(3)$ & $20.9694(7)$ & $110.993(2)$ & 3024.2 & & 7.5 \\
\hline UPULEX & 20.7890 & 10.2084 & 15.1091 & 110.8250 & 2997.01 & 4-aminobenzoic acid & 10 \\
\hline VACZIJ & $15.1550(3)$ & $10.2850(2)$ & $20.9056(5)$ & 109.86 & 3064.74 & dimethylsulfoxide & 7.35 \\
\hline VOJLIQ & $15.2196(10)$ & $10.2279(7)$ & $20.9321(14)$ & $110.9270(10)$ & 3043.45 & p-aminobenzoic acid & 5 \\
\hline XEGTAF & $10.601(4)$ & $26.402(9)$ & $15.586(6)$ & $93.145(6)$ & 4355.76 & 6'-Methoxycinchonan-9-ol & 16.5 \\
\hline YTYSII & 21.310 & 9.987 & 15.247 & 111.85 & 3011.8 & diethanolamine & 6.4 \\
\hline YIYSOO & 21.451 & 10.014 & 15.240 & 111.25 & 3051.12 & 1,5 -pentanediol & 6.2 \\
\hline ZIGZIY & $20.988(9)$ & $10.092(2)$ & $15.223(10)$ & $110.30(6)$ & 3024.13 & but-2-yne-1,4-diol & 7 \\
\hline$\beta$-CD-DMF & $15.500(3)$ & $9.794(2)$ & $21.689(5)$ & $90.138(3)$ & $3292.5(12)$ & dimethylformamide & 12.5 \\
\hline
\end{tabular}




\section{F Detailed description of the disorder} modelled in the high-pressure setup of $\alpha$-cyclodextrin used in the equilibration as
an infinite crystal

The setup consists of ten unit cells and $40 \alpha$-CD molecules (four molecules per unit cell). $\alpha$-CD molecules in the setup are labelled from 1 to 40 . The primary hydroxyl group of the glucose ring 1 (O6_1) exhibits (-)-gauche conformation (pointing outwards the $\mathrm{CD}$ cavity) in half the $\alpha$-CD molecules, and $(+)$ conformation (pointing inwards the cavity) in the other half. The $20 \alpha$-CD molecules which contain a (-) 06_1 group can also contain water molecules in the positions WC and WD; 32 out of the 40 available positions are occupied (16 WC and 16 WD).

\begin{tabular}{|c|c|c|c|c|c|c|c|}
\hline $\begin{array}{l}\alpha-C D \\
\text { index }\end{array}$ & $\begin{array}{c}\text { O6_1 group } \\
\text { conformation }\end{array}$ & $\begin{array}{c}\text { WC } \\
\text { occupied }\end{array}$ & $\begin{array}{c}\text { WD } \\
\text { occupied }\end{array}$ & $\begin{array}{l}\alpha-C D \\
\text { index }\end{array}$ & $\begin{array}{l}\text { O6_1 group } \\
\text { conformation }\end{array}$ & $\begin{array}{c}\text { WC } \\
\text { occupied }\end{array}$ & $\begin{array}{c}\text { WD } \\
\text { occupied }\end{array}$ \\
\hline 1 & $(-)$ & yes & yes & 21 & $(-)$ & yes & no \\
\hline 2 & $(-)$ & yes & no & 22 & $(+)$ & - & - \\
\hline 3 & $(-)$ & yes & yes & 23 & $(-)$ & yes & yes \\
\hline 4 & $(-)$ & yes & yes & 24 & $(-)$ & yes & yes \\
\hline 5 & $(+)$ & - & - & 25 & $(-)$ & no & yes \\
\hline 6 & $(+)$ & - & - & 26 & $(+)$ & - & - \\
\hline 7 & $(-)$ & yes & yes & 27 & $(-)$ & yes & yes \\
\hline 8 & $(-)$ & yes & no & 28 & $(+)$ & - & - \\
\hline 9 & $(+)$ & - & - & 29 & $(+)$ & - & - \\
\hline 10 & $(-)$ & yes & no & 30 & $(+)$ & - & - \\
\hline 11 & $(-)$ & yes & yes & 31 & $(+)$ & - & - \\
\hline 12 & $(-)$ & yes & yes & 32 & $(+)$ & - & - \\
\hline 13 & $(+)$ & - & - & 33 & $(+)$ & - & - \\
\hline 14 & $(-)$ & yes & yes & 34 & $(-)$ & no & yes \\
\hline 15 & $(+)$ & - & - & 35 & $(+)$ & - & - \\
\hline 16 & $(-)$ & no & yes & 36 & $(+)$ & - & - \\
\hline 17 & $(-)$ & yes & yes & 37 & $(+)$ & - & - \\
\hline 18 & $(+)$ & - & - & 38 & $(-)$ & no & yes \\
\hline 19 & $(+)$ & - & - & 39 & $(-)$ & yes & yes \\
\hline 20 & $(+)$ & - & - & 40 & $(+)$ & - & - \\
\hline
\end{tabular}




\section{G Detailed description of the disorder} modelled in the high-pressure setup of $\alpha$-cyclodextrin used in the equilibration as a finite crystal surrounded by water

See Appx. F for a detailed description on how the disorder is modelled. Then main difference here is a double number of $\alpha$-CD molecules.

\begin{tabular}{|c|c|c|c|c|c|c|c|}
\hline $\begin{array}{l}\alpha-C D \\
\text { index }\end{array}$ & $\begin{array}{c}\text { O6_1 group } \\
\text { conformation }\end{array}$ & $\begin{array}{c}\text { WC } \\
\text { occupied }\end{array}$ & $\begin{array}{c}\text { WD } \\
\text { occupied }\end{array}$ & $\begin{array}{l}\alpha-C D \\
\text { index }\end{array}$ & $\begin{array}{l}\text { O6_1 group } \\
\text { conformation }\end{array}$ & $\begin{array}{c}\text { WC } \\
\text { occupied }\end{array}$ & $\begin{array}{c}\text { WD } \\
\text { occupied }\end{array}$ \\
\hline 1 & $(-)$ & yes & yes & 41 & $(+)$ & - & - \\
\hline 2 & $(+)$ & - & - & 42 & $(+)$ & - & - \\
\hline 3 & $(-)$ & no & yes & 43 & $(-)$ & yes & yes \\
\hline 4 & $(-)$ & yes & yes & 44 & $(-)$ & yes & no \\
\hline 5 & $(-)$ & yes & yes & 45 & $(+)$ & - & - \\
\hline 6 & $(-)$ & yes & yes & 46 & $(+)$ & - & - \\
\hline 7 & $(-)$ & yes & yes & 47 & $(-)$ & yes & yes \\
\hline 8 & $(-)$ & no & yes & 48 & $(+)$ & - & - \\
\hline 9 & $(-)$ & no & yes & 49 & $(+)$ & - & - \\
\hline 10 & $(-)$ & yes & no & 50 & $(-)$ & yes & no \\
\hline 11 & $(+)$ & - & - & 51 & $(+)$ & - & - \\
\hline 12 & $(-)$ & yes & yes & 52 & $(-)$ & no & yes \\
\hline 13 & $(-)$ & yes & yes & 53 & $(+)$ & - & - \\
\hline 14 & $(+)$ & - & - & 54 & $(+)$ & - & - \\
\hline 15 & $(-)$ & yes & yes & 55 & $(+)$ & - & - \\
\hline 16 & $(-)$ & yes & yes & 56 & $(-)$ & yes & yes \\
\hline 17 & $(+)$ & - & - & 57 & $(+)$ & - & - \\
\hline 18 & $(-)$ & yes & yes & 58 & $(-)$ & yes & yes \\
\hline 19 & $(-)$ & yes & yes & 59 & $(-)$ & yes & yes \\
\hline 20 & $(-)$ & yes & yes & 60 & $(+)$ & - & - \\
\hline 21 & $(+)$ & - & - & 61 & $(+)$ & - & - \\
\hline 22 & $(+)$ & - & - & 62 & $(+)$ & - & - \\
\hline 23 & $(+)$ & - & - & 63 & $(+)$ & - & - \\
\hline 24 & $(+)$ & - & - & 64 & $(-)$ & yes & no \\
\hline 25 & $(+)$ & - & - & 65 & $(-)$ & yes & no \\
\hline 26 & $(-)$ & yes & yes & 66 & $(+)$ & - & - \\
\hline 27 & $(+)$ & - & - & 67 & $(-)$ & yes & yes \\
\hline 28 & $(+)$ & - & - & 68 & $(+)$ & - & - \\
\hline 29 & $(+)$ & - & - & 69 & $(-)$ & yes & yes \\
\hline
\end{tabular}


Appendix $G$

\begin{tabular}{cccc|cccc}
\hline \multicolumn{7}{c}{$\boldsymbol{c}$ (continue) } \\
index & $\begin{array}{c}\text { O6_1 group } \\
\text { conformation }\end{array}$ & $\begin{array}{c}\text { WC } \\
\text { occupied }\end{array}$ & $\begin{array}{c}\text { WD } \\
\text { occupied }\end{array}$ & $\begin{array}{c}\boldsymbol{\alpha} \text {-CD } \\
\text { index }\end{array}$ & $\begin{array}{c}\text { O6_1 group } \\
\text { conformation }\end{array}$ & $\begin{array}{c}\text { WC } \\
\text { occupied }\end{array}$ & $\begin{array}{c}\text { WD } \\
\text { occupied }\end{array}$ \\
\hline $\mathbf{3 0}$ & $(+)$ & - & - & $\mathbf{7 0}$ & $(-)$ & no & no \\
$\mathbf{3 1}$ & $(-)$ & yes & yes & $\mathbf{7 1}$ & $(+)$ & - & - \\
$\mathbf{3 2}$ & $(+)$ & - & - & $\mathbf{7 2}$ & $(+)$ & - & - \\
$\mathbf{3 3}$ & $(+)$ & - & - & $\mathbf{7 3}$ & $(-)$ & yes & yes \\
$\mathbf{3 4}$ & $(-)$ & yes & yes & $\mathbf{7 4}$ & $(-)$ & no & yes \\
$\mathbf{3 5}$ & $(+)$ & - & - & $\mathbf{7 5}$ & $(+)$ & - & - \\
$\mathbf{3 6}$ & $(-)$ & yes & yes & $\mathbf{7 6}$ & $(-)$ & yes & yes \\
$\mathbf{3 7}$ & $(+)$ & - & - & $\mathbf{7 7}$ & $(+)$ & - & - \\
$\mathbf{3 8}$ & $(-)$ & yes & yes & $\mathbf{7 8}$ & $(-)$ & yes & yes \\
$\mathbf{3 9}$ & $(+)$ & - & - & $\mathbf{7 9}$ & $(-)$ & yes & no \\
$\mathbf{4 0}$ & $(-)$ & no & yes & $\mathbf{8 0}$ & $(+)$ & - & - \\
\hline
\end{tabular}




\section{H Complete results from the simulations of $\alpha$-cyclodextrin infinite crystals}

In the main text, the conformation of the O6 groups is always defined as $(+)$ - or (-)-gauche, based on the value of the torsion angle O5-C5-C6-O6, as described in Fig. 3.6. The code used to analyse the MD trajectories uses instead the torsion angle C4-C5-C6-O5. According to this definition, when an O6 group is pointing outwards the $\alpha$-CD cavity, the torsion angle is smaller than $100^{\circ}$ and the conformation is $(+)$-gauche. When it is pointing inwards, the angle is larger $100^{\circ}$ and the conformation becomes trans-gauche. In the following, the conformation is given first according to the definition of the analysis script, and in parenthesis according to the conventional definition, followed by the terms in or out for clarity. Torsion angle values are given only according to the definition employed in the script.

\begin{tabular}{|c|c|c|c|c|c|}
\hline \multirow{3}{*}{$\begin{array}{l}\text { Simulation } \\
\text { O6 group }\end{array}$} & \multicolumn{5}{|c|}{ AP setup at $0.0001 \mathrm{GPa}$} \\
\hline & \multirow{2}{*}{ Conf. } & \multicolumn{2}{|c|}{ Torsion angle $\left(^{\circ}\right)$} & \multirow{2}{*}{$\begin{array}{c}\text { Length } \\
\text { (ns) }\end{array}$} & \multirow{2}{*}{$\begin{array}{c}\text { Occupancy } \\
(\%)\end{array}$} \\
\hline & & $\mu$ & $\sigma$ & & \\
\hline \multirow{4}{*}{ O6_1 } & $+(-$, out $)$ & - & - & - & - \\
\hline & $\operatorname{trans}(+$, in $)$ & 188.19 & 7.91 & 2375.392 & 98.97 \\
\hline & Intermediate & 281.23 & 7.38 & 0.088 & - \\
\hline & Other & & & 24.568 & 1.02 \\
\hline \multirow{4}{*}{ O6_2 } & $+(-$, out $)$ & 46.12 & 7.33 & 2368.156 & 98.67 \\
\hline & trans $(+$, in $)$ & 194.81 & 9.11 & 18.724 & 0.78 \\
\hline & Intermediate & - & - & - & - \\
\hline & Other & & & 13.168 & 0.55 \\
\hline \multirow{4}{*}{ O6_3 } & $+(-$, out $)$ & - & - & - & - \\
\hline & $\operatorname{trans}(+$, in $)$ & 197.81 & 9.25 & 2355.482 & 98.14 \\
\hline & Intermediate & 253.96 & 10.48 & 0.686 & 0.03 \\
\hline & Other & & & 43.880 & 1.83 \\
\hline \multirow{4}{*}{ O6_4 } & $+(-$, out $)$ & 45.13 & 7.50 & 2376.842 & 99.03 \\
\hline & $\operatorname{trans}(+$, in $)$ & 165.02 & 7.33 & 2.312 & 0.10 \\
\hline & Intermediate & 0.00 & - & - & - \\
\hline & Other & & & 20.894 & 0.87 \\
\hline \multirow{4}{*}{ O6_5 } & $+(-$, out $)$ & 55.79 & 7.73 & 2283.798 & 95.16 \\
\hline & trans $(+$, in $)$ & - & - & - & - \\
\hline & Intermediate & - & - & - & - \\
\hline & Other & & & 116.250 & 4.84 \\
\hline \multirow{4}{*}{ O6_6 } & $+(-$, out $)$ & 49.68 & 6.87 & 2382.820 & 99.28 \\
\hline & trans $(+$, in $)$ & - & - & - & - \\
\hline & Intermediate & - & - & - & - \\
\hline & Other & & & 17.228 & 0.72 \\
\hline
\end{tabular}




\begin{tabular}{|c|c|c|c|c|c|}
\hline \multirow{3}{*}{$\begin{array}{l}\text { Simulation } \\
\text { O6 group }\end{array}$} & \multicolumn{5}{|c|}{ HP setup at $0.65 \mathrm{GPa}$} \\
\hline & \multirow{2}{*}{ Conf. } & \multicolumn{2}{|c|}{ Torsion angle $\left(^{\circ}\right)$} & \multirow{2}{*}{$\begin{array}{c}\text { Length } \\
\text { (ns) }\end{array}$} & \multirow{2}{*}{$\begin{array}{c}\text { Occupancy } \\
(\%)\end{array}$} \\
\hline & & $\mu$ & $\sigma$ & & \\
\hline \multirow{4}{*}{ O6_1 } & $+(-$, out $)$ & 52.70 & 8.57 & 1921.918 & 48.05 \\
\hline & trans $(+$, in $)$ & 189.96 & 8.34 & 2050.088 & 51.25 \\
\hline & Intermediate & 292.21 & 8.89 & 3.286 & 0.08 \\
\hline & Other & & & 24.788 & 0.62 \\
\hline \multirow{4}{*}{ O6_2 } & $+(-$, out $)$ & 43.47 & 7.86 & 3973.134 & 99.33 \\
\hline & trans $(+$, in $)$ & 183.69 & 9.22 & 3.092 & 0.08 \\
\hline & Intermediate & 229.32 & 18.27 & 0.492 & 0.01 \\
\hline & Other & & & 23.362 & 0.58 \\
\hline \multirow{4}{*}{ O6_3 } & $+(-$, out $)$ & 60.81 & 11.61 & 74.548 & 1.86 \\
\hline & $\operatorname{trans}(+$, in $)$ & 195.35 & 11.99 & 3854.434 & 96.36 \\
\hline & Intermediate & 257.43 & 9.93 & 33.720 & 0.84 \\
\hline & Other & & & 37.378 & 0.93 \\
\hline \multirow{4}{*}{ O6_4 } & $+(-$, out $)$ & 45.99 & 7.50 & 3970.082 & 99.25 \\
\hline & $\operatorname{trans}(+$, in $)$ & - & - & - & - \\
\hline & Intermediate & - & - & - & - \\
\hline & Other & & & 29.998 & 0.75 \\
\hline \multirow{4}{*}{ O6_5 } & $+(-$, out $)$ & 55.58 & 8.73 & 3863.250 & 96.58 \\
\hline & $\operatorname{trans}(+$, in $)$ & 167.71 & 14.57 & 5.392 & 0.13 \\
\hline & Intermediate & 231.94 & 9.11 & 99.094 & 2.48 \\
\hline & Other & & & 32.344 & 0.81 \\
\hline \multirow{4}{*}{ O6_6 } & $+(-$, out $)$ & 49.48 & 7.05 & 3969.966 & 99.25 \\
\hline & $\operatorname{trans}(+$, in $)$ & 186.63 & 14.74 & 3.290 & 0.08 \\
\hline & Intermediate & 265.45 & 23.30 & 3.144 & 0.08 \\
\hline & Other & & & 23.680 & 0.59 \\
\hline
\end{tabular}




\begin{tabular}{|c|c|c|c|c|c|}
\hline \multirow{3}{*}{$\begin{array}{l}\text { Simulation } \\
\text { O6 group }\end{array}$} & \multicolumn{5}{|c|}{$\mathrm{AP}$ setup at $0.65 \mathrm{GPa}$} \\
\hline & \multirow{2}{*}{ Conf. } & \multicolumn{2}{|c|}{ Torsion angle $\left({ }^{\circ}\right)$} & \multirow{2}{*}{$\begin{array}{c}\text { Length } \\
\text { (ns) }\end{array}$} & \multirow{2}{*}{$\begin{array}{c}\text { Occupancy } \\
(\%)\end{array}$} \\
\hline & & $\mu$ & $\sigma$ & & \\
\hline \multirow{4}{*}{ O6_1 } & $+(-$, out $)$ & 73.91 & 8.46 & 267.128 & 11.13 \\
\hline & $\operatorname{trans}(+$, in $)$ & 188.77 & 8.04 & 2110.498 & 87.94 \\
\hline & Intermediate & 287.23 & 5.86 & 0.166 & 0.01 \\
\hline & Other & & & 22.256 & 0.93 \\
\hline \multirow{4}{*}{ O6_2 } & $+(-$, out $)$ & 45.12 & 7.55 & 2276.858 & 94.87 \\
\hline & $\operatorname{trans}(+$, in $)$ & 196.38 & 9.71 & 109.410 & 4.56 \\
\hline & Intermediate & - & - & - & - \\
\hline & Other & & & 13.780 & 0.57 \\
\hline \multirow{4}{*}{ O6_3 } & $+(-$, out $)$ & - & - & - & - \\
\hline & $\operatorname{trans}(+$, in $)$ & 196.85 & 10.35 & 2355.584 & 98.15 \\
\hline & Intermediate & 227.27 & 4.03 & 4.650 & 0.19 \\
\hline & Other & & & 39.814 & 1.66 \\
\hline \multirow{4}{*}{ O6_4 } & $+(-$, out $)$ & 44.74 & 7.62 & 2381.860 & 99.24 \\
\hline & $\operatorname{trans}(+$, in $)$ & - & - & - & - \\
\hline & Intermediate & - & - & - & - \\
\hline & Other & & & 18.188 & 0.76 \\
\hline \multirow{4}{*}{ O6_5 } & $+(-$, out $)$ & 55.00 & 8.32 & 2381.016 & 99.21 \\
\hline & $\operatorname{trans}(+$, in $)$ & 137.66 & 6.77 & 0.232 & 0.01 \\
\hline & Intermediate & 229.80 & 7.77 & 2.270 & 0.09 \\
\hline & Other & & & 16.530 & 0.69 \\
\hline \multirow{4}{*}{ O6_6 } & $+(-$, out $)$ & 50.19 & 6.99 & 2339.602 & 97.48 \\
\hline & $\operatorname{trans}(+$, in $)$ & 170.90 & 7.59 & 0.130 & 0.01 \\
\hline & Intermediate & 229.73 & 45.76 & 44.020 & 1.83 \\
\hline & Other & & & 16.296 & 0.68 \\
\hline
\end{tabular}




\begin{tabular}{|c|c|c|c|c|c|}
\hline \multirow{3}{*}{$\begin{array}{c}\text { Simulation } \\
\text { O6 group }\end{array}$} & \multicolumn{5}{|c|}{$\mathrm{HP}$ setup at $0.0001 \mathrm{GPa}$} \\
\hline & \multirow{2}{*}{ Conf. } & \multicolumn{2}{|c|}{ Torsion angle $\left(^{\circ}\right)$} & \multirow{2}{*}{$\begin{array}{c}\text { Length } \\
\text { (ns) }\end{array}$} & \multirow{2}{*}{$\begin{array}{c}\text { Occupancy } \\
(\%)\end{array}$} \\
\hline & & $\mu$ & $\sigma$ & & \\
\hline \multirow{4}{*}{ O6_1 } & $+(-$, out $)$ & 55.33 & 9.04 & 1796.190 & 44.90 \\
\hline & trans $(+$, in $)$ & 184.74 & 8.48 & 2158.454 & 53.96 \\
\hline & Intermediate & 276.53 & 8.17 & 11.606 & 0.29 \\
\hline & Other & & & 33.830 & 0.85 \\
\hline \multirow{4}{*}{ O6_2 } & $+(-$, out $)$ & 47.79 & 8.20 & 3956.594 & 98.91 \\
\hline & trans $(+$, in $)$ & 191.60 & 11.35 & 18.864 & 0.47 \\
\hline & Intermediate & 239.27 & 11.89 & 0.178 & - \\
\hline & Other & & & 24.444 & 0.61 \\
\hline \multirow{4}{*}{ O6_3 } & $+(-$, out $)$ & 60.33 & 9.76 & 175.530 & 4.39 \\
\hline & $\operatorname{trans}(+$, in $)$ & 197.89 & 9.86 & 3721.044 & 93.02 \\
\hline & Intermediate & 254.05 & 11.22 & 55.110 & 1.38 \\
\hline & Other & & & 48.396 & 1.21 \\
\hline \multirow{4}{*}{ O6_4 } & $+(-$, out $)$ & 48.15 & 7.83 & 3969.238 & 99.23 \\
\hline & $\operatorname{trans}(+$, in $)$ & 0.00 & - & - & - \\
\hline & Intermediate & 0.00 & - & - & - \\
\hline & Other & & & 30.842 & 0.77 \\
\hline \multirow{4}{*}{ O6_5 } & $+(-$, out $)$ & 58.92 & 8.13 & 3926.342 & 98.16 \\
\hline & $\operatorname{trans}(+$, in $)$ & 195.01 & 12.96 & 31.468 & 0.79 \\
\hline & Intermediate & 282.07 & 6.57 & 1.428 & 0.04 \\
\hline & Other & & & 40.842 & 1.02 \\
\hline \multirow{4}{*}{ O6_6 } & $+(-$, out $)$ & 49.61 & 7.10 & 3964.592 & 99.11 \\
\hline & $\operatorname{trans}(+$, in $)$ & 212.28 & 33.25 & 12.674 & 0.32 \\
\hline & Intermediate & 275.47 & 10.22 & 0.792 & 0.02 \\
\hline & Other & & & 22.022 & 0.55 \\
\hline
\end{tabular}




\section{Technical description of the diamond anvil} cells

\section{I.1 Square cell}

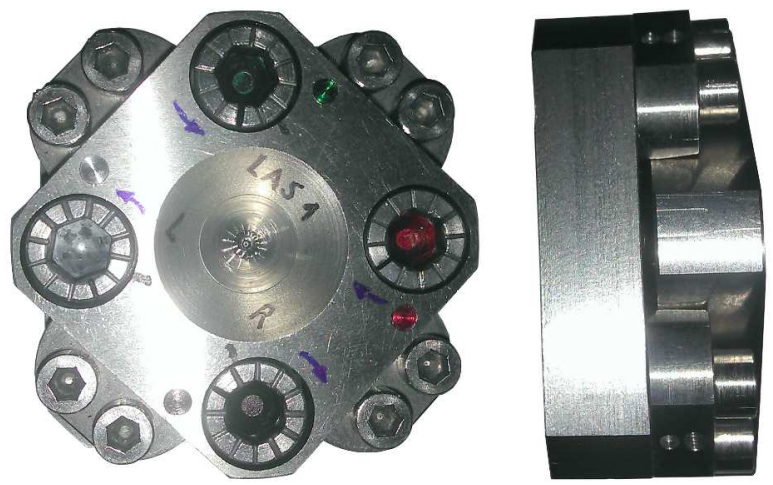

Culet size $(\mu \mathrm{m})$ 550

Window opening angle $\left(^{\circ}\right) \quad 90$

Diamond cut

Baking plate

Ahsbahs

Diamond fixation

Steel

Dimensions ( $\mathrm{mm}$ )

Screws

$60 \times 60 \times 21$

Used for

Crystal A of $\beta$-CD form II (§3.2.1) Pure ${ }^{t} \mathrm{BA}$ form II $(\S 6.2 .4 .1)$ 


\section{I.2 Triangular cell}

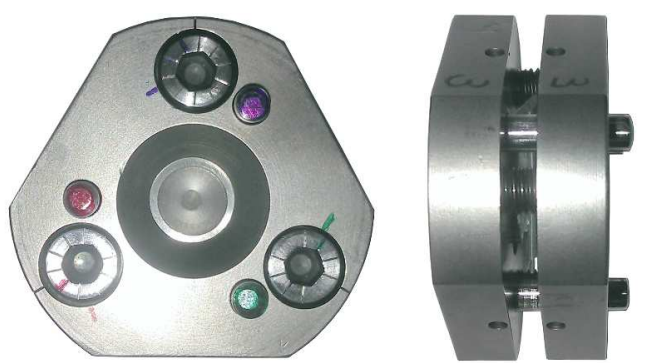

Culet size $(\mu \mathrm{m})$

600 (Model 1)

800 (Model 2)

Window opening angle $\left(^{\circ}\right) \quad 90$

Diamond cut

Bhoeler-Almax

Baking plate

Tungsten carbide

Diamond fixation

Glued

Dimensions ( $\mathrm{mm}$ )

$43 \times 37 \times 20$

(Model 1) Crystal B of $\beta$-CD form II (§3.2.1)

(Model 1) ${ }^{t}$ BA 5.80-hydrate (\$6.2.3.1)

(Model 2) $\alpha$-CD compression study (\$5.2.1)

(Model 2) ${ }^{t}$ BA 5.65-hydrate (§6.2.2.1) 


\section{I.3 $90 / 100^{\circ}$ cell}
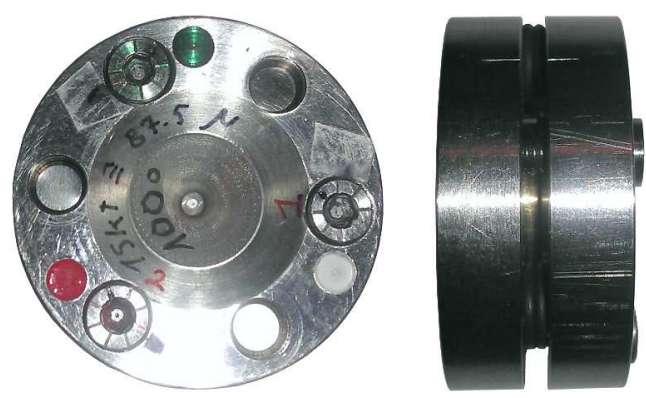

$\begin{array}{ll}\text { Culet size }(\boldsymbol{\mu m}) & 700 \\ \text { Window opening angle }\left(^{\circ}\right) & 90 / 100 \\ \text { Diamond cut } & \text { Ahsbahs }\left(90^{\circ} \text { side }\right) \\ & \text { Flat }\left(100^{\circ} \text { side }\right) \\ \text { Baking plate } & \text { Tungsten carbide } \\ \text { Diamond fixation } & \text { Glued } \\ \text { Dimensions }(\mathbf{m m}) & 40(\varnothing) \times 18 \\ \text { Used for } & \text { Crystal } \mathbf{C} \text { of } \beta \text {-CD form III }(\S 3.2 .1) \\ & \alpha \text {-CD at } 0.20 \mathrm{GPa}(\S 5.2 .1)\end{array}$

This DAC has been custom designed by Hans Ahsbahs (Margburg) in 2011. Differently from the other two models, is asymmetric. One side is similar to the previous ones, with a steel body, tungsten carbide backing plate with an opening angle of $90^{\circ}$, and a glued Bhoeler-Almax diamond. The other side is equipped with a flat diamond, which has a larger support area, hence it can be mounted on a backing plate with a wider

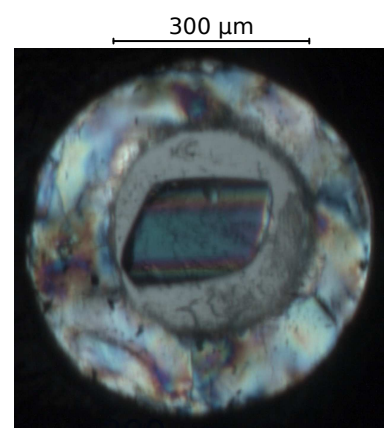
opening angle of $100^{\circ}$. The main advantage of this cell is that it allows for better completeness during data collection.

Data quality can be further improved by removing the diffraction and reducing the absorption and shading from the gasket. In practice, this is done by drilling a large hole in the gasket $(\sim 600 \mu \mathrm{m}$ for $700 \mu \mathrm{m}$ culets), filling it with epoxy resin, and then drilling the resin to the desired size of the sample chamber (Fig. A.1). In this way, the metallic

Figure A.1: Epoxy ring placed inside the hole drilled on a brass gasket (in black).

gasket provides structural support for the cell without interacting with the X-ray beam.

\section{I.3.1 Optimising data collection with the $90 / 100^{\circ}$ cell}

The software available to correct for the shadowing effect observed when collecting diffraction data using a DAC considers that both sides of the 
cell are symmetric. Under this assumption, it is not possible to take full advantage of the $90 / 100^{\circ} \mathrm{DAC}$, because correcting for a window opening of $100^{\circ}$ does not remove completely the shadows cast by the $90^{\circ}$ side, while correcting for a window opening of $90^{\circ}$ removes the extra data collected thanks to the wider opening of $100^{\circ}$ on the other side. A Python program called SomBRA ${ }^{1}$ (from the Spanish word for shadow), was written to address this situation.

Sombra was designed under the same principles than $\mathrm{SHADE}^{2}$, and works on the output of the latter. SHADE takes as input a RAW file output by SAINT ${ }^{3}$ and calculates the angles of incidence and diffraction of each reflection from the corresponding direction cosines, the metric tensor of the unit cell, the orientation matrix of the crystal, and the orientation in which the DAC is mounted on the diffractometer. These angles are used to remove reflections which lye in the shadowed areas, but the complete list can also be directly output to apply the correction with Sombra. In addition, correlation coefficients, which can be used to asses the quality of reflections that are close to the edge of the shadowed area, are passed from the RAW file onto SHADE's output.

SOMBRA takes as input the output from SHADE and the user is asked to input the opening angles of each side of the DAC (as half-opening angles), referred as the side which faces the incoming beam $(B)$ and the side which faces the detector $(D)$. Angles smaller than the opening of the windows can be introduced for a more conservative result, especially if problematic reflections are expected on the edges of the shadowed regions. The program then removes any reflection which fulfils any of the following criteria:

- The angle of incidence is larger than $B$.

- The angle of incidence is larger than $B-2^{\circ}$ and its correlation coefficient is larger than 0.3 .

- The angle of diffraction is larger than $D$.

\footnotetext{
${ }^{1}$ Source code available on request.

${ }^{2}$ S. Parsons, Shade, The University of Edinburgh, Edinburgh, United Kingdom, 2005.

${ }^{3}$ Bruker-AXs, SAINT+ Integrating Engine v8.18c, Bruker-AXs, Madison, Wisconsin, USA, 2011.
} 
- The angle of diffraction is larger than $D-2^{\circ}$ and its correlation coefficient is larger than 0.3 .

A new RAW file is finally produced, containing only the accepted reflections.

The performance of SomBRA has been tested on data collected on a sample of $\alpha$-CD form I at $0.20 \mathrm{GPa}$ (Ch. 5). Data were collected in four independent runs. The first run $(\mathbf{R} 1)$ was collected with the $100^{\circ}$ window facing the incoming beam, while the other three (R2, R3, and R4) were collected with the $90^{\circ}$ window facing the beam. All runs were first integrated in SAINT with a cutoff for the window opening of $100^{\circ}$, to remove only the smallest shadow possible. Three post-integration shadow corrections were applied:

- a) Using SHADE with a cutoff of $86^{\circ}$.

- b) Using SHADE with a cutoff of $96^{\circ}$.

- c) Using Sombra with a cutoff of 86 and $96^{\circ}$ for the narrow and the wide window, respectively.

The number of reflections remaining after each shadow correction, with respect to the total number of integrated reflections, for each run, has been compared (Table A.1). Runs like R1 allow to collect more frames, but the shadowed region in those frames is larger. On the other hand, runs like R2-4 provide fewer frames, but with smaller shadows on them. In terms of integrated reflections, $\mathbf{R} 1$ yields more reflections than R2-4. In terms of corrected reflections, $\mathbf{R} 1$ yields more reflections when using SHade, although the situation is inverted when using Sombra. Ultimately, the number of unique reflections is more important. It is assumed here that SOMBRA performs a complete removal of all shadows, and it is interesting to realise that the number of unique reflections after the corrections coincide with the one obtained by applying SHADE using as cutoff the opening of the window facing the detector. This means that, at least in this particular case, runs like R2-4 yield more unique reflections than runs like $\mathbf{R} \mathbf{1}$, although the difference is small-3010 against 2861 reflections, respectively. 


\begin{tabular}{|c|c|c|c|c|}
\hline \multirow{2}{*}{ Run } & \multirow{2}{*}{$\begin{array}{c}\text { Shadow } \\
\text { corrections }\end{array}$} & \multicolumn{3}{|c|}{ Number of reflections } \\
\hline & & Integrated & Corrected & Unique \\
\hline \multirow{3}{*}{ R1 } & SHADE $86^{\circ}$ & & 9163 & 2861 \\
\hline & SHADE $96^{\circ}$ & 10698 & 10240 & 3042 \\
\hline & SOMBRA $86 / 96^{\circ}$ & & 9515 & 2861 \\
\hline \multirow{3}{*}{ R2 } & SHADE $86^{\circ}$ & & 8858 & \multirow{9}{*}{$\begin{array}{l}2795 \\
3010 \\
3010\end{array}$} \\
\hline & SHADE $96^{\circ}$ & 9934 & 9622 & \\
\hline & Sombra $86 / 96^{\circ}$ & & 9621 & \\
\hline \multirow{3}{*}{ R3 } & SHADE $86^{\circ}$ & \multirow{3}{*}{9914} & 8850 & \\
\hline & SHADE $96^{\circ}$ & & 9621 & \\
\hline & SOMBRA $86 / 96^{\circ}$ & & 9621 & \\
\hline \multirow{3}{*}{ R4 } & SHADE $86^{\circ}$ & \multirow{3}{*}{9845} & 8791 & \\
\hline & SHADE $96^{\circ}$ & & 9553 & \\
\hline & Sombra $86 / 96^{\circ}$ & & 9552 & \\
\hline
\end{tabular}

Table A.1: Number of reflections at different points of the data processing.

Based on these results, both SHADE and Sombra perform equally well, although it is of paramount importance to properly choose the cutoffs for the correction. If using SHADE, the cutoff should correspond to the opening of the window facing the detector, which implies that scans of the front and back side of the DAC must be integrated separately (§1.1.3). SomBRA can correct for both sides at the same time; the appropriate cutoff for each side, along with the orientation of the windows, should be given. Although not implemented yet, it is possible to modify the program to apply the correction to several scans that have been integrated together. Integration of several scans together is desirable, although not always possible, to perform more accurate absorption corrections (§1.1.3). 
